\title{
ANÁLISE DA INTERAÇÃO SOLO NÃO-HOMOGÊNEO/ESTRUTURA VIA ACOPLAMENTO MEC/MEF
}

\author{
VALÉRIO SILVA ALMEIDA
}

Tese apresentada à Escola de Engenharia de São Carlos da Universidade de São Paulo, como parte dos requisitos para a obtenção do título de Doutor em Engenharia de Estruturas.

ORIENTADOR: Prof. Assoc. João Batista de Paiva 


\section{AGRADECIMENTOS}

Sobretudo, a DEUS por ter me dado saúde e me feito forte para enfrentar os obstáculos que surgiram em toda a minha vida.

Ao meu compreensivo orientador Prof. João Batista de Paiva pela atenção e dedicação.

A Fundação de Amparo à Pesquisa do Estado de São Paulo FAPESP - pelo suporte técnico e financeiro concedido a este projeto.

Agradeço aos meus "pontos de referência" de vida, minha esposa Ariadine S. Lechner e minha filha Cecília L. Almeida.

Sou grato também aos professores que contribuíram de maneira singular em alguns pontos do trabalho e, sobretudo, no meu amadurecimento humano e profissional: Prof. Carlos Armando M. Duarte, Profa. Helena M.C.C. Antunes, Prof. Humberto B. Coda, Prof. Nelson Aoki, Prof. Walter Savassi e Prof. Wilson Sérgio Venturini.

A todos os companheiros que mantive neste período, em especial a Jeselay H.C. dos Reis, Alexandre Botta, Ângelo Mendonça, Luciano S.G. Leite e Luttgardes Neto, e também aos amigos do futebol.

Agradeço a todos do Departamento de Estruturas da EESC/USP, em especial à Nadir Minatel e ao Masaki.

Enfim, felizmente, inúmeras são as pessoas que deveriam ser citadas que aqui não caberiam. Ficam tácitas neste texto e, sobretudo, concretas em minhas demonstrações de agradecimentos. 


\section{RESUMO}

ALMEIDA, V.S. (2003). Análise da interação solo não-homogêneo/estrutura via acoplamento MEC/MEF. São Carlos. 192p. Tese (Doutorado) Escola de Engenharia de São Carlos, Universidade de São Paulo.

O estudo do comportamento mecânico do complexo sistema advindo da interação entre solo/subestrutura/superestrutura é o tema do trabalho. Neste contexto, a representação do maciço é feita usando-se o método dos elementos de contorno (MEC) em abordagem 3D, de maneira que se possa simular o maciço com características mecânicas não-homogêneas, além de se considerar uma camada de apoio indeslocável a distâncias prescritas a priori e condição de aderência perfeita. A subestrutura também é representada via MEC tridimensional, a qual está imersa dentro deste meio heterogêneo. A infra e a superestrutura são modeladas empregando o método dos elementos finitos (MEF), com o uso de elementos estruturais reticulares e elementos laminares. São apresentados alguns exemplos em que se valida a formulação e outros que demonstram a potencialidade e a necessidade de se empregar a formulação para a melhor análise do complexo fenômeno em estudo. Por fim, demonstra-se a obrigatoriedade de se otimizar a formulação, empregando-se duas grandes ferramentas numéricas: o paralelismo e o emprego de um adequado método de resolução de sistemas esparsos.

Palavras-chave: interação solo/estrutura; método dos elementos de contorno; solo não-homogêneo; acoplamento MEC/MEF; paralelismo; resolução de sistemas lineares esparsos. 


\section{ABSTRACT}

ALMEIDA, V.S. (2003). Analysis of nonhomogeneous soil-structure interaction using BEM-FEM coupling. São Carlos. 192p. Thesis (Doctoral) - São Carlos School of Engineering, University of São Paulo.

The analysis of the soil-structure system interaction is a vast field of interest in the area of civil engineering. A realistic representation of its behaviour is a complex numerical task due to its extremely variable mechanical behaviour. Thus, in the present research, the soil is considered a non-homogeneous continuum supported by a rigid and adhesive interface and modelled by boundary element method via Kelvin solution in 3D space. The foundation is also modelled by this above-mentioned modelling technique. The raft foundation and the superstructure are represented by finite shell and 3D frame elements. In order to estimate the accuracy and the potentiality of the proposed numerical formulation, some examples are validated when compared to similar approaches, and others simulations are presented to stress the necessity of coupling the non-homogeneous soilfoundation-radier-superstructure system as a whole. Finally, to acquire numerical time efficiency, it is shown that it is imperative to apply parallel processing and sparse techniques for the solution of the final system.

Keywords: soil-structure interaction; boundary element method; nonhomogeneous soil; coupling BEM-FEM; parallel processing; solution of sparse linear equations. 


\section{Sumário}

Lista de figuras vi

Lista de tabelas $\quad$ xiii

$\begin{array}{ll}\text { Revisão bibliográfica } & 1\end{array}$

1.1 Considerações iniciais 1

1.2 Organização do texto 1

1.3 Revisão bibliográfica 3

Capítulo 2: Método dos elementos de contorno 15

2.1 Introdução 15

2.2 Relações básicas do problema elástico linear

2.3 Representação das equações de equilíbrio no contorno

2.4 A solução fundamental tridimensional de Kelvin 
2.5.2 Representação algébrica das equações integrais de contorno

2.5.3 Integração numérica e subelementação

2.6 Sub-região

2.7 Exemplos numéricos dos casos tridimensionais

2.7.2 Viga elástica solicitada à flexão

\section{Capítulo 3: Formulação do solo não-homogêneo enrijecido por} elemento de fundação

3.1 Introdução

3.2 Maciço estratificado pelo método da "rigidez sucessiva" 
3.4 Avaliação do número de operações envolvidas a priori

4.1 Introdução

4.2 Formulação do método dos elementos finitos

4.3 Superestrutura formada por elementos laminares

4.3.1 Efeito de membrana

4.3.2 Efeito de flexão

4.3.3 Matriz de rigidez do elemento laminar no plano tridimensional

4.4 Superestrutura formada por edifícios 3D sujeitos às ações verticais e horizontais

4.5 Acoplamento MEC/MEF

4.6 Exemplos numéricos

4.6.1 Placa quadrada simplesmente apoiada 
5.2 Lâmina quadrada apoiada sobre meio semi-infinito

5.3 Camada finita com variação linear do módulo de elasticidade

5.4 Lâmina fina sobre uma base não-deformável

5.5 Lâmina quadrada sob um meio homogêneo de base rígida

5.6 Edifício sobre radier apoiado em um maciço não-homogêneo

5.7 Uma estaca no meio finito homogêneo

5.8 Uma estaca no meio finito não-homogêneo

5.9 Lâmina quadrada apoiada sobre nove estacas

5.10 Cobertura apoiada em "radier" com e sem estacas processamento distribuído

6.2.3 Medida de desempenho 
6.2.5.1 Whitaker sem estaca

6.2.5.2 Lâmina quadrada apoiada sobre meio semi-infinito

6.2.5.3 Uma estaca no meio finito não-homogêneo

6.3 Resolução de sistemas lineares esparsos

6.3.1 Aplicações numéricas

6.3.1.1 Acoplamento edifício/radier/solo 


\section{Lista de figuras}

Figura 2.1 - Representação de um sólido com condições de contorno mistas.

Figura 2.2 - Domínio acrescido de complemento infinitesimal para o caso 3D.

Figura 2.3 - Valores dos termos da matriz C para três casos de cantos ortogonais.

Figura 2.4 - a) Geometria do elemento e sistemas de coordenadas; b) Coordenadas oblíquas.

Figura 2.5 - Funções interpoladoras adotadas.

Figura 2.6 - Subelementação regular e coordenadas locais e globais.

Figura 2.7 - Gráficos das integrais no elemento quasi-singular; i) ponto fora a $1 / 40 \mathrm{~L}$ ii) ponto fora a $1 / 10 \mathrm{~L}$; iii) ponto fora a $1 / 2 \mathrm{~L}$ ( $\mathrm{L}$ é maior lado).

Figura 2.8 - Subelementação progressiva para a posição " $P$ " e parâmetros necessários.

Figura 2.9 - Definição de parâmetros para a integração singular.

Figura 2.10 - Representação da integração do domínio do elemento pelo seu lados.

Figura 2.11 - a) Representação de um sólido não-homogêneo. b) Condições de interface entre regiões. 
Figura 2.12 - Sólido apoiado em sua base (plano $X_{1} X_{2}$ e $X_{3}=0$ ) e com valor de $E=1$ e $v=0,25$.) Rede adotada para o contorno e coordenadas de pontos de interesse.

Figura 2.13 - Subelementações empregadas.

Figura 2.14 - Geometria e valores elásticos da viga engastada no plano $\left(X_{1} X_{2}\right.$ e $\left.X_{3}=0\right)$, e configuração da rede.

Figura 2.15 - Estaca submetida à força vertical e parâmetros físicos e geométricos.

Figura 2.16 - Configurações da rede adotada para o solo e a estaca.

Figura 2.17 - Deslocamentos horizontais ao longo do eixo da estaca.

Figura 2.18 - Forças de contato horizontais ao longo do eixo da estaca.

Figura 2.19 - Elemento de fundação submetido à força vertical, parâmetros físicos e geométricos e vista da rede empregada para simular a estaca ou o tubulão.

Figura 2.20 - Deslocamentos verticais ao longo do eixo da estaca e do tubulão.

Figura 2.21 - Forças de contato ao longo do eixo da estaca.

Figura 2.22 - Forças de contato na região da ponta do tubulão e da estaca.

Figura 3.1 - Maciço estratificado sob forças de superfície e contornos de base, topo e lateral.

Figura 3.2 - Estrato genérico com duas estacas e seus parâmetros de topo, base e fuste.

Figura 3.3 - Esquema do maciço estratificado estaqueado. 
Figura 3.4 - Número de operações dividido por $\mathrm{N}^{3}$ versus número de camadas, para dois procedimentos: i) Método da "rigidez sucessiva" (MRS) e ii) Método da subregião (SR).

Figura 4.1 - Geometria e os 18 graus de liberdade do elemento laminar da junção DKT/FF no sistema local $O x_{1} x_{2}$.

Figura 4.2 - Relação entre sistema local e global de um plano qualquer no espaço 3D.

Figura 4.3 - Vetores auxiliares para cálculo dos co-senos diretores de um elemento.

Figura 4.4 - Forças de superfície e cargas nodais equivalentes de um elemento.

Figura 4.5 - Variação linear do deslocamento transversal e da força de superfície no interior do elemento finito.

Figura 4.6 - Forças de superfície e cargas nodais equivalentes.

Figura 4.7 - Placa quadrada apoiada e parâmetros geométricos e físicos.

Figura 4.8 - Geometria e redes adotadas, sendo $E=300, v=0,3, L=R=300, h=3,0$ (espessura).

Figura 4.9 - Deslocamentos em $\mathrm{X}_{3}$ ao longo do contorno (A-B).

Figura 4.10 - Valores de momentos fletores na direção y' ao longo do contorno (C-D).

Figura 5.1 - a) esquema estrutural do modelo; b) rede usada para discretizar a lâmina; c) parâmetros físicos e geométricos do conjunto lâmina/solo.

Figura 5.2 - Rede usada para discretizar a superfície livre do solo. a) Rede 1;b) Rede 2. 
Figura 5.4 - Forças de superfície do contato lâmina/solo sobre o eixo S.

Figura $5.5-$ i) estrato finito sob carregamento uniforme circular ii) rede usada para o solo.

Figura 5.6 - a) esquema da lâmina sobre meio indeformável; b) rede de um quarto da lâmina; c) parâmetros físicos e geométricos do solo e da lâmina ; d) rede usada para o solo.

Figura 5.7 - a) estrato finito sob carregamento uniforme; b) rede usada para o solo e para a lâmina.

Figura 5.8 - a) corte da estrutura e os casos considerados; b) planta do modelo edifício/radier; c) planta baixa do edifício; d) tabelas dos parâmetros dos elementos de barra do edifício.

Figura 5.9 - Deslocamentos verticais ao longo do corte AA.

Figura 5.10 - Deslocamentos verticais no radier para o caso b.

Figura 5.11 - Momentos fletores $\mathrm{M}_{11}$ sobre todo o radier para o caso $b$.

Figura 5.12 - Momentos fletores $\mathrm{M}_{22}$ sobre todo o radier para o caso $b$.

Figura 5.13 - Tensões de contato $\left(\sigma_{33}\right)$ em toda região comum ao radier e ao solo para o caso $b$.

Figura 5.14 - Distribuição de tensões verticais $\left(\sigma_{33}\right)$ no solo ao longo do corte BB para o caso $c$.

Figura 5.15 - Distribuição de deslocamentos $\left(\mathrm{u}_{3}\right)$ no solo ao longo do corte BB para o caso $C$. 
Figura 5.16 - Curva deslocamentos horizontais (metros) versus andares do nó mestre do edifício para o caso $b$.

Figura 5.17 - Distribuição de momentos fletores $(\mathrm{kN} \cdot \mathrm{m})$ para um pilar ao longo de dois 130 andares e de uma viga em comum a eles.

Figura 5.18- a) estaca sobre força vertical em meio indeslocável; b) perspectiva da discretização empregada; c) redes usadas para simular a superfície livre do solo; d) redes usadas para representar a estaca; e) uma perspectiva da rede da estaca.

Figura 5.19- Forças de contato cisalhante entre estaca/solo para diversos $\lambda$ e $H / L$, para a rede $\mathrm{i} / \mathrm{iv}$.

Figura 5.20 Deslocamentos verticais das estacas para diversos $\lambda$ e $\mathrm{H} / \mathrm{L}$, para a rede i/iv.

Figura 5.21- Deslocamentos da estaca para os 3 tipos de redes do solo, empregando a rede iv, $H / L=1,5$ e $\lambda=1000$.

Figura 5.22- Forças de contato cisalhante entre estaca/solo para os 3 tipos de redes, empregando a rede iv, $\mathrm{H} / \mathrm{L}=1,5$ e $\lambda=1000$.

Figura 5.23- Deslocamentos da estaca com os 3 tipos de redes do solo, empregando as 3 redes da estaca, para $H / L=1,5$ e $\lambda=1000$.

Figura 5.24- Forças de contato cisalhante entre estaca/solo para os 3 tipos de redes, empregando as 3 redes da estaca, para $H / L=1,5$ e $\lambda=1000$.

Figura 5.25- Estaca sujeita a força vertical em meio não-homogêneo e configurações do maciço.

Figura 5.26- Forças de contato verticais entre estaca/solo para diferentes estratificações.

Figura 5.27- Deslocamentos verticais na estaca para 4 casos de rigidez do solo. 
Figura 5.28- Forças de contato cisalhantes verticais entre estaca/solo para 4 casos de rigidez do solo.

Figura 5.29- Representação da lâmina sobre maciço homogêneo estaqueado apoiado em meio indeslocável, e seus parâmetros físicos e geométricos.

Figura 5.30 Redes usadas para discretizar a lâmina, as estacas e a superfície do solo.

Figura 5.31- Deslocamentos da superfície de contato lâmina/solo/estaca.

Figura 5.32- Deslocamentos das estacas ao longo do fuste sem lâmina.

Figura 5.33- Forças de contato cisalhantes entre solo/estaca sem lâmina.

Figura 5.34- Representação da cobertura sobre maciço homogêneo estaqueado. Rede empregada para o solo idem a figura 5.21 .

Figura 5.35- Deslocamento vertical na seção da cobertura paralelo ao eixo $\mathrm{X}_{1}$ para diferentes fatores de espessura do solo, sem estacas.

Figura 5.36- Deslocamentos da cobertura no corte CD considerando diversas espessuras do radier, com o solo estaqueado e estaca 3 afunilada.

Figura 6.1 - Esquema simplificado da técnica da decomposição em domínio.

Figura 6.2 - Modelo de criação e transferência de dados em árvore entre processadores.

Figura 6.3 - Estrutura de dados para a matriz gerada no acoplamento edifício/radier/solo.

Figura 6.4 - Estrutura de dados para a matriz gerada em um problema MEC/MEC 2D.

Figura 6.5 - Estrutura de dados para a matriz gerada em um problema MEC/MEC 2D. 
Figura 6.6 - Estrutura plana com carga unitária uniformemente distribuída ao longo do contorno da direita com $E=1, v=1 / 3$.

Figura 6.7 - Tempo de resolução para os diferentes métodos de resolução. 


\section{Lista de tabelas}

Tabela 2.1:

Deslocamentos $u_{3}$ dos pontos $A, B$ e $C$ do sólido, em unidade de comprimento.

Tabela 2.2:

Tensões $\sigma_{33}$ do sólido, em unidade de tensão.

Tabela 2.3:

Linha elástica do sólido analisado $(\mathrm{cm})$.

Tabela 2.4:

Tensão vertical $\left(\sigma_{33}\right)$ para 3 situações.

Tabela 4.1:

Deslocamentos, momentos e erros relativos no centro da placa para 3 tipos de redes.

Tabela 5.1:

Valores de deslocamentos e forças de contato verticais para o centro da lâmina.

Tabela 5.2:

Erro porcentual dos deslocamentos centrais da superfície.

Tabela 5.3:

Resultados de deslocamentos e momentos fletores para pontos da lâmina.

Tabela 5.4:

Deslocamento vertical $(\mathrm{m})$ do ponto $\mathrm{A}$. 
Tabela 5.5:

Forças normais (MN) nos pilares do andar térreo para os vários casos de rigidez do solo. Sinal positivo indica compressão nos pilares.

Tabela 5.6:

Porcentagem das forças normais distribuídas nos pilares de estremidade, intermediário e de canto.

Tabela 6.1:

Para 2 camadas do solo.

Tabela 6.2:

Para 3 camadas do solo.

Tabela 6.3:

Para 4 camadas do solo.

Tabela 6.5:

Para 3 camadas do maciço.

Tabela 6.6:

Para 3 camadas do maciço.

Tabela 6.7:

Tempo (minutos) para resolução do sistema linear com 4431 graus de liberdade e com $26 \%$ de coeficientes não-nulos.

Tabela 6.8:

Tempo (segundos) para resolução do sistema linear com 1312 graus de liberdade e com $33 \%$ de coeficientes não-nulos. 


\section{Capítulo 1}

\section{Considerações iniciais e revisão bibliográfica}

\subsection{Introdução}

Este trabalho apresenta os resultados obtidos pela análise numérica de um complexo problema da engenharia civil: a interação solo/estrutura.

Neste sentido, o seguinte texto expõe as formulações dos dois métodos numéricos aplicados na construção do problema como um todo. Apresentando as potencialidades que cada método possui para sua aplicação em seu determinado campo da análise estrutural. Deste modo, os capítulos posteriores desenvolvem as formulações do método dos elementos de contorno (MEC) e do método dos elementos finitos (MEF) focados aos campos de aplicação do problema em estudo, e mostra-se a técnica empregada para acoplar as diferentes formulações.

\subsection{Organização do texto}

O primeiro capítulo aborda os principais trabalhos apresentados na literatura científica a respeito das formulações existentes para se avaliar numericamente o comportamento de um maciço heterogêneo com espessura finita, considerando-se a presença ou não de elementos de fundação.

O segundo capítulo trata de maneira sucinta a formulação do método dos elementos de contorno. Destaca-se que este desenvolvimento já está voltado 
para o campo de aplicação desse método no presente trabalho. No final do capítulo, quatro exemplos são avaliados para se averiguar a formulação desenvolvida.

O terceiro capítulo traz o desenvolvimento da formulação desenvolvida, via MEC, para simular a heterogeneidade do maciço de solos com a inclusão dos elementos de fundação nesse meio não-homogêneo. Compara-se, também, o número de operações em ponto flutuante necessário para o método desenvolvido com o convencionalmente empregado, que é a técnica da subregião.

No capítulo 4, é apresentado o equacionamento do MEF, as hipóteses admitidas para o elemento de lâmina e para a formulação do edifício, entretanto não se apresenta o equacionamento para a formação dos elementos de lâmina nem do edifício, citando-se as referências que detalham este expediente. $\mathrm{Na}$ segunda parte do capítulo, se desenvolve a técnica empregada para se realizar o acoplamento entre o MEC e MEF, e no final do capítulo apresenta-se dois exemplos para exemplificar a formulação do MEF implementada.

O capítulo 5 traz os exemplos numéricos avaliados com o uso da formulação desenvolvida. Assim, vários exemplos são apresentados, tentandose, na medida do possível, mostrar o potencial da ferramenta desenvolvida.

O sexto capítulo tem o intuito de apresentar duas importantes ferramentas empregadas para otimizar o tempo de resposta do código: o paralelismo e o uso de uma técnica eficiente para resolver sistemas esparsos. Assim, é apresentada uma pequena introdução sobre o uso da computação distribuída no campo dos métodos numéricos e os procedimentos usados para paralelizar o código, e exemplos avaliados no computador paralelo do centro de processamento da escola de Engenharia de São Carlos. Por fim, faz-se um breve comentário sobre as técnicas de resolução de sistemas esparsos, avaliando, para diferentes técnicas, os resultados de tempo de processamento sequencial de alguns exemplos obtidos com o emprego do MEF, ou MEC ou do acoplamento MEC/MEF. 


\subsection{Revisão bibliográfica}

A interação solo/estrutura representa um sistema mecânico integrado. A análise, geralmente avaliada entre os meios, ou mais especificamente, entre a superestrutura, a infra-estrutura, a subestrutura e o maciço de solos, é tratada de forma independente.

Esta simplificação advém do alto grau de complexidade para se avaliar o fenômeno mecânico único. Pois, cada um dos sistemas por si já representa um vasto campo de estudo, quer na variabilidade de parâmetros físicos e geométricos, quer nas correspondentes idealizações dos modelos mecânicos. Somando-se a isto, os diferentes ramos de interesse e de conhecimento dos pesquisadores nesta área são voltados para referenciais diferentes.

Por um lado, o engenheiro geotécnico analisa a complexa integração entre a subestrutura e o maciço de solos, sem considerar as mudanças de configurações que possam ocorrer na superestrutura e que venha a levar a um estado de tensões não previsto no sistema subestrutura/maciço. Em contrapartida, o engenheiro estrutural está voltado para os fenômenos que ocorrem na superestrutura, sem levar em conta os efeitos que o meio de apoio está sujeito quando da absorção das ações, e nas posteriores modificações da superestrutura que possa acarretar.

Além disso, um fenômeno comum que ambos os pesquisadores não têm dado tanto ênfase é sobre o efeito de grupo que altera o estado de tensões do sistema envolvido. Assim, além de se avaliar o sistema superestrutura/ subestrutura/maciço de solos de forma desacoplada, considera-se este sistema livre do efeito de vizinhança, o que pode levar a estados de deformações e/ou tensões não previstos nos projetos envolvidos.

Sobre a avaliação qualitativa e quantitativa da superestrutura de forma isolada, os modelos usados para representar seus fenômenos têm sido melhorados dia-a-dia. Pois, com o desenvolvimento das ferramentas computacionais e a utilização dos métodos discretos, as incorporações de modelos mecânicos mais sofisticados são realizadas comumente. Dentre os 
trabalhos que estão inseridos neste contexto, é imprescindível citar: BARROS (2002), BATHE (1982), BEZERRA (1995), DUARTE et al. (2000), MENDONCA (2002), MESQUITA (1998), OÑATE (1995), PELETEIRO (1996), RIOS (1991).

No que se refere à análise do maciço de solos, em geral, o solo é assumido como sendo homogêneo, isótropo e elástico linear e semi-infinito. Entretanto, na maioria dos casos, a consideração da camada do solo apoiada em uma camada indeslocável a uma distância infinita não representa a real configuração do maciço, uma vez que a consideração da não-homogeneidade e da base rígida a uma distância prescrita indica as condições mais próximas da encontrada na natureza. Por isso, na literatura as técnicas propostas para modelar o meio finito do solo estão embasadas em quatro diferentes modelos.

No primeiro, o meio contínuo é substituído por um sistema de molas equivalente e discreto, também conhecido como modelo de Winkler. Caso haja estaca, ela é idealizada como um elemento de viga com ou sem efeito da deformação axial apoiado por uma série de molas discretas que representam o solo. As maiores vantagens na aplicação deste modelo são sua simplicidade e relativa facilidade para implementação computacional. Como maior desvantagem, tem-se a dificuldade de se escolher os módulos de reação da mola para uma certa combinação de tamanho de estaca e tipo de solo. Geralmente esses parâmetros são estimados por correlações empíricas e podem levar a soluções incertas e imprecisas. Nessa linha, desenvolvem-se os trabalhos de CHEUNG \& ZIENKIEWICZ (1965), RANDOPH \& WROTH (1979), WITT (1984), LEE (1993) e MYLONAKIS \& GAZETAS (1998). Esses dois últimos autores apresentam um método simples para cálculo de recalques e tensões de uma estaca ou de grupos de estacas imersas em um meio nãohomogêneo. O solo ao redor da estaca é representado por um modelo generalizado de Winkler onde a rigidez de cada cota do meio não-homogêneo é avaliada empiricamente.

A segunda técnica parte do manuseio das equações advindas da teoria da elasticidade aplicada a um meio contínuo, homogêneo e elástico, e nas 
superfícies de contato entre as camadas adjacentes são aplicadas as condições de equilíbrio e compatibilidade para se obterem as expressões que configurem o problema não - homogêneo. Esta técnica apresenta a desvantagem de que as soluções encontradas são aplicadas em alguns problemas, pois de forma geral as equações não são resolvidas. Assim, têm-se os trabalhos pioneiros de BURMISTER $(1943,1945)$, que aplica a técnica de transformação de integral para a obtenção das soluções semi-analíticas para os deslocamentos e as tensões de um meio não-homogêneo sem considerar elementos de fundação, para duas e três camadas, quando se aplica uma força concentrada na superfície livre do meio. Ele parte das equações de equilíbrio e compatibilidade do problema elástico tridimensional e, via condições de contorno entre as camadas, recai em uma integral que envolve funções de Bessel e em uma expressão no denominador cuja resolução é impraticável. Então, Burmister exprime esse denominador como uma série de termos exponenciais que devem ser explicitados para os diversos casos de combinação entre os parâmetros físicos das respectivas camadas do solo.

POULOS (1967) aplica as soluções de BURMISTER, obtendo os fatores de influência para os casos de carregamentos de linha, de faixa e setorial. CHAN et al. (1974) generalizam o procedimento de BURMISTER para o caso de se considerar força vertical e/ou horizontal aplicada em um ponto no interior de duas camadas. Assim como BURMISTER, a expressão que aparece no denominador é aproximada por uma série de funções exponenciais que deve ser obtida numericamente para as diversas relações entre os módulos de rigidez e os coeficientes de Poisson das camadas adjacentes.

DAVIES \& BANERJEE (1978) repetem a técnica utilizada por CHAN et al. (1974). Entretanto, calculam as integrais de forma mais precisa via métodos de quadraturas e deixam as funções de deslocamentos explícitas para as diversas combinações dos parâmetros elásticos. Eles concluem que essas soluções fundamentais, obtidas semi-analiticamente, têm alta convergência para casos simples. Contudo, para problemas mais complexos que envolvam 
meios não-homogêneos, tal como para a análise de grupos de estacas localizadas em diferentes estratos, é necessário utilizar as soluções numéricas, que são obtidas com muito trabalho e que não são muito vantajosas para o caso em que se deseja muita precisão, devido à complexidade das expressões integrais.

GIBSON (1967) apresenta a solução para o campo de deslocamento vertical $(w)$ sujeito unicamente a forças verticais na superfície livre do meio isótropo, elástico, com variação linear da rigidez crescente ao longo da profundidade, com o índice de Poisson constante, e com uma base indeslocável de apoio. Mais tarde, GIBSON (1974) estende seu trabalho de 1967 considerando o meio anisótropo, particularizado para o caso em que os três módulos elásticos $\left(E_{x}, E_{z}\right.$ e $\left.G_{x z}\right)$ são independentes e variam linearmente com a profundidade. Outro trabalho também importante nesta linha é o de UESHITA \& MEYERHOF (1968).

A terceira técnica é denominada de método da camada finita (MCF). $\mathrm{O}$ método pode ser empregado para se reduzir o problema tridimensional a um outro que envolva duas dimensões mediante a combinação da técnica da transformada de Fourier e o método dos elementos finitos. O MCF permite uma implementação computacional fácil e eficiente para soluções elásticas do solo homogêneo ou não, isótropo ou anisótropo. Para o caso de se incorporar elementos de estaca, ele pode ser empregado considerando-se as condições de compatibilidade e equilíbrio entre as superfícies dos dois meios. Uma desvantagem do MCF é que ele apenas pode ser empregado em problemas elásticos. Como referência, citam-se os trabalhos pioneiros de SMALL \& BOOKER (1984), BOOKER et al. (1989), LEE \& SMALL (1991), SOUTHCOTT \& SMALL (1996) e TA \& SMALL (1998). Uma variante do MCF é o método da camada infinita $(\mathrm{MCl})$ apresentado em CHEUNG et al. (1988).

A quarta linha de pesquisa tem se utilizado dos potentes métodos numéricos, mais precisamente do emprego do método dos elementos finitos (MEF) e o método dos elementos de contorno (MEC). O MEF é, em geral, a 
mais versátil e poderosa ferramenta usada na resolução de problemas da mecânica computacional. No entanto, para o caso de ser aplicada na análise de problemas de domínio infinito - caso da análise de solos - ela é uma ferramenta difícil na preparação dos dados, onerosa para o seu armazenamento e para a resolução do sistema final, principalmente para o caso de se usar uma formulação com elementos tridimensionais. Isto porque o método necessita de uma representação de todo o domínio. Assim, poucos pesquisadores têm empregado o MEF na análise de maciços homogêneos e não-homogêneos, citando-se o trabalho de OTTAVIANI (1975) e CHOW \& TEH (1991).

O MEC tem-se mostrado a mais eficiente e prática ferramenta para a análise de problemas de domínio infinito, devido às características peculiares das funções ponderadoras que já contemplam as condições de contorno atendidas a grandes distâncias. Um ramo de pesquisadores tem empregado a solução de MINDLIN (1936) - a qual avalia os campos de deslocamentos dentro de um meio semi-infinito e homogêneo, quando se aplica uma força unitária dentro do meio - para o caso da análise de um meio elástico apoiado em um plano indeslocável, a uma distância h da superfície do solo. Assim, tem-se o modelo simplificado e prático de Steinbrenner (POULOS (1967)), que emprega a solução de Mindlin calculando os deslocamentos de um determinado ponto e subtraindo-se este do deslocamento surgido na cota indeslocável $\left(\rho_{x}^{\text {Steinhremer }}=\rho_{x}^{\text {Mindlin }}-\rho_{h}^{\text {Mindlin }}\right)$, onde $\rho_{x}$ é o deslocamento na cota $x$ e $\rho_{h}$ é o deslocamento na cota do plano indeslocável. POULOS (1968a) estende o trabalho do ano anterior considerando uma estaca carregada axialmente, e em POULOS (1968b) ele aplica a formulação de Steinbrenner para grupos de estacas. POULOS (1979) avalia a influência de uma estaca dentro de um meio utilizando a expressão $\left(\rho_{i j}=\frac{d}{E_{s}} \cdot I_{i j} \cdot p_{j}\right)$, onde $\rho_{i j}$ é o deslocamento do solo do elemento $i$ devido a um fator de influência de tensão $p_{j}$ do elemento $j, d$ é o diâmetro da estaca, $I_{i j}$ é o fator de influência de deslocamento obtido por Mindlin, e $E_{s}$ é o módulo de elasticidade do meio. Para um solo não-homogêneo 
ele, por conveniência, altera os valores do módulo de Young ao longo da profundidade do solo e conclui que os resultados estão de acordo com os obtidos via MEF. Entretanto, para casos em que as estacas estão imersas em meios onde as camadas inferiores são mais compressíveis que as superiores, os resultados não são tão aceitáveis.

Também neste sentido, pode-se empregar o MEC via solução de Mindlin, discretizando-se a superfície do indeslocável e impondo-se as restrições requeridas nestes pontos.

Entretanto, a aplicação do MEC utilizando-se a solução fundamental de Mindlin, mesmo com a introdução da cota do plano indeslocável, só é válida na representação de um único meio homogêneo, não sendo possível, dentro da teoria da elasticidade, introduzir outros meios com características físicas diferentes, em função das condições de contorno já impostas a priori por MINDLIN (1936) em sua formulação. E, conforme POULOS (1979), para certas condições de não homogeneidade do meio com estaca, os resultados de deslocamentos não são adequados.

Deste modo, é necessário utilizar o MEC com as respostas de domínio infinito. A solução de Kelvin (LOVE (1944)) fornece a base para as mais gerais formulações sobre um corpo isótropo de forma arbitrária. Essa solução é a mais empregada para acoplar diversas regiões com propriedades diferentes, equilibrando-as e compatibilizando-as nas superfícies de fronteira, mantendo assim a continuidade do meio heterogêneo.

BANERJEE (1976), BANERJEE \& DAVIES (1977) e MAIER \& NOVATI (1987) aplicam o MEC utilizando a solução fundamental de Kelvin para simular o meio finito heterogêneo. O primeiro autor apresenta uma formulação direcionada para a análise de solo do tipo Gibson e se considera a influência das estacas imersas nesse meio. Tanto o solo quanto as estacas são representados por sólidos tridimensionais e se considera o radier rígido acoplado à superfície do solo. BANERJEE \& DAVIES (1977) aplicam o procedimento de BANERJEE (1976), mas já possibilitando a análise dos 
maciços com as camadas descritas com quaisquer valores de módulo de Young e coeficiente de Poisson. Entretanto, a superestrutura acoplada ao maciço é também definida como um bloco rígido.

MAIER \& NOVATI (1987) desenvolveram um método alternativo para a análise de solos estratificados mediante uma técnica denominada de "método da rigidez sucessiva" via MEC. Cada estrato é tratado como uma sub-região, e, utilizando-se as condições de equilíbrio e compatibilidade existentes entre os estratos adjacentes, é possível transferir a rigidez da camada inferior para sua adjacente superior. Desta forma, chega-se à última camada com uma matriz que já incorporou as influências de todas as outras camadas inferiores. Neste trabalho, avalia-se este procedimento em exemplos bidimensionais. Como conclusão chega-se à matriz final gerada bem-condicionada, cuja resolução exige esforço computacional menor que o empregado pela técnica convencional de sub-regiões no MEC. O método é aplicável na introdução de outras subregiões, como na colocação de túneis e de elementos de fundação.

Alguns outros trabalhos usam formulações que não foram classificadas dentre as quatro apresentadas, em virtude da escassa literatura sobre os procedimentos empregados. Cita-se o modelo de CHIN \& CHOW (1990), que emprega o MEC; entretanto a solução fundamental usada é a obtida no artigo de CHAN et al. (1974). FRASER \& WARDLE (1976) empregam uma técnica, que é uma variante do MEF, mas usando as funções ponderadoras de meio semi-infinito, denominada de aproximação por elemento de superfície, em que é possível considerar a não-homogeneidade do solo, e somente a porção da sua região onde existe carregamento precisa ser discretizada. Analisa-se, então, a interação entre fundação formada por radier flexível e solo não-homogêneo sobre uma base rígida.

SADECKA (2000) considera um modelo para o solo, que é uma generalização direta da teoria de Vlasov para diversas camadas, em que são introduzidas funções de peso não-lineares para os deslocamentos ao longo da profundidade dos estratos, e admite-se a influência da placa apoiada sobre a 
superfície livre do solo usando o MEF e o método dos elementos infinitos para a região fora de contato entre placa-solo.

PAN (1997) aplica o MEC tridimensional para a análise de meios semiinfinitos estratificados, mas usa as funções de Green para não ter que empregar as técnicas de sub-regiões para os diversos meios com propriedades físicas diferentes. A formulação é combinada com o método da matriz propagadora, e as suas soluções são expressas em termos de integrais infinitas, que envolvem funções de Bessel. Entretanto esta formulação não permite a incorporação de outros elementos enrijecedores no maciço, e as camadas do solo só podem ser estratificadas horizontalmente.

Há também uma outra linha de trabalhos em que alguns autores procuram integrar os procedimentos empregados no modelo de Winkler e os modelos contínuos. Isto é feito introduzindo continuidade entre os elementos discretos de mola por meio de elementos estruturais, ou aplicando nos modelos contínuos algumas simplificações, mas calibradas com valores de deslocamentos ou tensões reais. Nessa linha, têm-se os modelos de: fundação de Hetenyi's, encontrado em HETENYI (1950); fundação de Pasternak, visto em KERR (1964) e WANG et al. (2001); fundação generalizada, encontrado em KERR (1964); e fundação de Kerr, visto no trabalho de KERR (1965).

No Brasil, alguns pesquisadores também têm analisado 0 comportamento dos meios estratificados com a camada do indeslocável em uma posição finita. Assim, acontece no trabalho de AOKI \& LOPES (1975) que avaliam as tensões e deslocamentos para fundações profundas conforme apresentado em POULOS (1968b); e de REIS (2000), que utiliza o procedimento de AOKI \& LOPES (1975) aplicado para fundações rasas.

ROMANEL et al. (2000) aplicam o MEF no domínio transformado de Fourier, o que faz com que o problema 3D seja analisado em 2D, e avaliado o comportamento mecânico de fundações do tipo radier sobre um meio finito. ROMANEL \& KUNDU (1990) aplicam as funções de Green para a análise da 
interação solo de multicamadas-estrutura em casos bidimensionais dinâmicos via MEF.

MOURA (1995) desenvolveu uma metodologia para a análise da interação solo-estrutura em que o edifício $3 \mathrm{D}$ foi modelado empregando-se a formulação matricial com elementos de barras para as vigas e os pilares, e em que as lajes são consideradas como diafragmas rígidos. Empregou-se a técnica da subestruturação para a simulação do edifício. A fundação é composta por elementos de sapata e o solo homogêneo de camada finita é modelado conforme procedimento de AOKI \& LOPES (1975).

GUSMÃO (1990) aplica os procedimentos de AOKI \& LOPES (1975) à análise de recalques de edifícios planos apoiados sobre solo de espessura finita e não-homogêneo.

MESQUITA \& CODA (2000) e LEITE et al. (2001) analisam o solo apoiado em uma camada finita aplicando o MEC bidimensional. Assim, o primeiro trabalho citado avalia o comportamento de um pórtico plano apoiado sobre duas sapatas, cada uma das quais colocada sobre um meio finito com propriedades físicas diferentes. No segundo trabalho, os autores inserem no meio finito um elemento enrijecedor que representa a estaca.

ANTUNES \& IWAMOTO (2000) aplicam os procedimentos de POULOS (1968b) para a modelagem do meio não-homogêneo apoiado em uma camada finita. Aplicam-se então incrementos de carga, e a ponta da estaca será mobilizada após toda a mobilização do fuste ocorrer.

HOLANDA JR. (1998) faz análise da interação solo-estrutura para edifícios de concreto armado sobre fundações diretas, em que o solo é suposto homogêneo e apoiado a uma cota com plano indeslocável, empregando o modelo proposto por POULOS (1967).

Como se pode perceber, muitas diferentes técnicas foram e são empregadas para a modelagem do meio não-homogêneo associado ou não a elementos de fundação. 
Entretanto, a grande maioria dos procedimentos descritos apresentam certas restrições. Algumas formulações não permitem ou tornam complicada a inclusão de elementos de fundações; outras só são válidas para certas condições de simetria quer de ações, quer de forma. Têm-se também aquelas que apenas permitem análises elásticas ou alguma restrição cinemática, por exemplo, a não-inclusão de deslizamento relativo entre os diferentes meios em contato.

É fato que a modelagem de meio finito não-homogêneo representa um problema da mecânica dos sólidos altamente complexo, em virtude de que não houve controle tecnológico para a formação de seu material, como acontece, por exemplo, na confecção do aço ou mesmo do concreto armado. Mas, sim, sua formação se deu em função das diversas condições de intemperismo sobre as rochas, o que tornou o meio altamente heterogêneo e anisótropo, de aparência caótica e com descontinuidades presentes ao longo de seu volume.

Dessa forma, para uma representação mais próxima das condições reais do solo, é necessário empregar as leis constitutivas adequadas, simular as configurações de maneira que se moldam ao longo do maciço, procurando: a) integrar os diferentes subespaços - cada qual com suas características isótropas ou anisotrópicas semelhantes - para formarem o meio como um todo; b) permitir a inclusão dos diversos elementos de fundações existentes com suas leis de contato existentes; c) garantir o acoplamento das superestruturas; e d) possibilitar a modelagem de túneis.

Neste contexto, o emprego dos métodos numéricos é a ferramenta mais completa e prática para a possível simulação destas condições. Como já foi salientado, o MEF representa uma técnica muito custosa para esta modelagem, em virtude da obrigatoriedade de discretização em elementos tridimensionais do solo, finito na vertical, mas infinito nas direções radiais, até as regiões consideradas imobilizadas pelas ações aplicadas. Por outro lado, o uso do MEC, além de diminuir a discretização do problema em uma dimensão, ou seja, discretização do problema 3D com elementos planos, ele emprega soluções 
ponderadoras que já são atendidas a distâncias não perturbadas. E, também, é possível encontrar com facilidade, na literatura corrente, os procedimentos para se incorporarem as diversas leis reológicas e cinemáticas para se modelar com mais realismo o complexo meio, que é o maciço de solos. Assim, o emprego do MEC com a solução fundamental de Kelvin é um dos possíveis caminhos para esta modelagem.

O grande gargalo da representação numérica computacional na simulação de problemas complexos quer via métodos de domínio (MEF), quer de contorno (MEC), é a enorme geração de dados que deverão ser armazenados e operacionalizados dentro do computador, e cuja análise, dependendo do modelo empregado, pode levar dias para a obtenção das respostas.

Mas, hoje em dia, o crescimento da tecnologia aplicada nos componentes microeletrônicos dos computadores causou a diminuição do custo, maior velocidade de operações de dados em ponto flutuante por segundo, sistemas mais eficientes de transferência de dados entre memórias virtuais e físicas (sistemas de barramentos), aumento dos espaços para armazenamento de dados nas diversas memórias existentes, e também, mais recentemente, maior facilidade para o emprego de conjunto de computadores, resolvendo um mesmo problema: computação paralela. Tudo isso tem levado os pesquisadores a aplicarem com mais ênfase os métodos numéricos no campo da mecânica dos sólidos, já que eles exigem um tratamento matemático e de implementação mais fácil, sem a perda de generalidade do modelo a ser simulado.

Neste contexto, NOOR (1997) apresenta um excelente estudo sobre o impacto que tais máquinas têm causado no campo da análise estrutural, enfatizando os avanços na microeletrônica e na tecnologia de redes, no paradigma da interação homem-máquina e técnicas, avanços em ambientes de programação, algoritmos numéricos e estratégias computacionais para os novos sistemas de computação. 
Assim, pelo que foi exposto ao longo desta revisão, fica claro o emprego do MEC no presente trabalho, método que emprega a solução tridimensional de Kelvin, de modo que a representação do maciço de solos não-homogêneo será tomada conforme o procedimento proposto por MAIER \& NOVATI (1987), mas estendido à análise tridimensional e inovando esta técnica com a inclusão de sub-regiões, também tridimensionais, que simulem os elementos de fundação estacas, sapatas, tubulões, buracos, túneis - e que venham a ultrapassar ou não as diferentes camadas. Entretanto, essa formulação não considera o deslizamento relativo entre estes meios. Para avaliar o efeito do sistema superestrutura/subestrutura/maciço de solos, é realizado o acoplamento do sistema solo/subestrutura com a superestrutura, simulada via MEF com elementos convencionais de lâmina, sendo todo o sistema considerado com a teoria elastostática linear.

Em face da grande quantidade de dados gerados para todo o modelo e do alto número de operações numéricas envolvidas ao longo da montagem do sistema final, emprega-se a técnica de decomposição em domínio, de modo que os diferentes subdomínios (sub-regiões) são montados simultaneamente via uso de sistemas computacionais em paralelo, do tipo memória distribuída.

No acoplamento MEC/MEF, o sistema final - dependendo da ligação entre as estruturas - pode gerar uma matriz esparsa, e neste caso é aplicada uma rotina específica para este tipo especial de matriz, uma vez que isto traz também uma maior rapidez para a resolução do sistema linear. 


\section{Capítulo 2}

\section{MÉTODO DOS ELEMENTOS DE CONTORNO}

\subsection{Introdução}

Este capítulo tem o intuito de apresentar sucintamente a formulação do método dos elementos de contorno (MEC) para a análise de problemas tridimensionais em teoria estática linear. Salienta-se que o desenvolvimento foi direcionado para as considerações adotadas para a presente formulação empregada ao longo do trabalho.

Para um entendimento mais completo e geral do método, citam-se os trabalhos de BESKOS (1987), VENTURINI (1988), BREBBIA \& DOMINGUEZ (1989), CODA (2000) e MENDONÇA (2002).

\subsection{Relações básicas do problema elástico linear}

O estado de tensões de um elemento infinitesimal dentro de um sólido de domínio finito $\Omega$ e contorno $\Gamma$ é representado por um tensor de segunda ordem composto por nove componentes. Mas quando se aplicam as equações de equilíbrio de momento neste elemento infinitesimal, desde que não haja momento de corpo, verifica-se a simetria das tensões cisalhantes, ou seja, em termos indiciais:

$$
\sigma_{i j}(s)=\sigma_{j i}(s) \quad i, j=1,2,3
$$


Empregando as três condições de equilíbrio de forças neste elemento infinitesimal, pode-se expressar as equações diferenciais que regem o problema da seguinte forma:

$$
\sigma_{i j, j}(s)+b_{i}(s)=0 \quad i, j=1,2,3
$$

onde " $s$ " é um ponto do domínio do corpo e $b_{i}$ representa as forças de massa (por unidade de volume).

É possível relacionar o estado de tensões de um elemento infinitesimal de faces ortogonais com suas componentes localizadas em uma faceta inclinada conhecendo-se apenas os co-senos diretores $\left(n_{j}\right)$ da normal desta faceta com relação às faces ortogonais do elemento, onde $n_{j}=\cos \left(n, x_{j}\right), j$ varia de 1 a 3 indicando as três direções cartesianas e $n$ é o vetor normal da faceta inclinada. Assim, pode-se obter as componentes de forças de superfície do contorno " $S$ " (vide figura 2.1). Considerando-se o equilíbrio do tetraedro - formado pelas faces ortogonais e a face inclinada - e admitindo-se que as forças de massa dão contribuição de ordem superior, as forças de superfície são correlacionadas com as tensões de domínio mediante a expressão:

$$
p_{i}(S)=\sigma_{i j}(s) \cdot \eta_{j} \quad i, j=1,2,3
$$

Quando sobre o sólido atuam ações externas, este sofre mudança de posição e/ou de dimensões, representadas pelas componentes de deslocamentos $u_{i}(s)$. Esses deslocamentos podem ser decompostos em duas parcelas.

A primeira, denominada de deslocamento de corpo rígido, não introduzindo nenhuma variação de forma e de dimensão no corpo, e assim não mobilizando o aparecimento de tensões no sólido.

A segunda parcela do deslocamento, associada à mudança de forma e de dimensões, leva ao aparecimento das tensões dentro do sólido. Esta parcela é expressa em termos da taxa de variação de uma configuração deformada com a 
indeformada, medindo-se, assim, a deformação linear específica (ou alongamento linear) e/ou angular (ou distorção) do corpo.

Esta taxa de variação de deslocamento é expresso por um tensor gradiente da deformação que, para o campo dos pequenos deslocamentos e seus gradientes suficientemente pequenos, pode-se apenas considerar os termos de primeira ordem, e definir assim as deformações lineares (homogêneas) do corpo. Este tensor, denominado de tensor de deformações específicas de Cauchy, é relacionado com as componentes do deslocamento por:

$$
\varepsilon_{i j}(s)=\frac{1}{2} \cdot\left(u_{i, j}(s)+u_{j, i}(s)\right) \quad i, j=1,2,3
$$

onde $u_{i}$ são os deslocamentos ao longo da direção $i$, e $\varepsilon_{i j}$ são as deformações específicas associadas ao elemento infinitesimal localizado no ponto " $s$ ".

O corpo sob efeito de ações externas quer de forças de campo, quer de forças de superfície ou deslocamentos impostos, sofre deformações que irão se desenvolver ao longo do corpo conforme a natureza de seu material. Assim, admitindo-se o campo de deslocamentos e de tensões contínuos e unívocos, é possível relacionar os dois estados mediante as relações constitutivas.

Para o caso mais simples da relação entre tensão e deformação, as hipóteses admitidas para a natureza do material são de: homogeneidade, que admite que todo o material tenha as mesmas propriedades físicas; isotropia, que garante que em qualquer ponto do corpo as propriedades são as mesmas sem referência à direção; e linearidade elástica entre tensão e deformação.

Com essas hipóteses, e admitindo que não haja mudanças de temperaturas, a fórmula indicial a seguir expressa as leis constitutivas usadas para esse corpo:

$$
\sigma_{i j}(s)=\lambda \cdot \delta_{i j} \cdot \varepsilon_{k k}(s)+2 \cdot \mu \cdot \varepsilon_{i j}(s) \quad i, j, k=1,2,3
$$

onde $\lambda$ e $\mu$ são as constantes de Lamé, que podem ser expressas em função dos módulos de elasticidade longitudinal, transversal e o coeficiente de Poisson, respectivamente, E,G e $v$. 


$$
\lambda=\frac{v \cdot E}{(1+v) \cdot(1-2 v)}, \quad \mu=G=\frac{E}{2(1+v)}
$$

E podendo-se também escrever a equação 2.5 da seguinte maneira:

$$
\sigma_{i j}(s)=c_{i j k l} \varepsilon_{k l} \quad i, j, k, l=1,2,3
$$

onde $c_{i j k l}$ é o tensor constitutivo de quarta ordem.

Com o uso das relações de equilíbrio (2.2), junto com as equações que relacionam deslocamento e deformações (2.4), mais as relações constitutivas entre tensão e deformação (2.5), podem ser expressas as equações de equilíbrio em termos de deslocamento, relações conhecidas como equações de Navier:

$$
u_{i, j j}(s)+\frac{1}{1-2 \cdot v} \cdot u_{j, j i}(s)+\frac{b_{i}(s)}{G}=0 \quad i, j=1,2,3
$$

onde se é possível aplicar três diferentes tipos de condições de contorno nas equações de Navier:

1) as condições de Fronteira de Dirichlet:

$$
u_{i}=\bar{u}_{i} \quad \text { onde } \quad \Gamma=\Gamma_{u} \quad i=1,2,3
$$

2) as condições de Fronteira de Neumann:

$$
p_{i}=\bar{p}_{i} \quad \text { onde } \quad \Gamma=\Gamma_{p} \quad i=1,2,3
$$

3) as condições de Fronteira mista:

$$
\begin{array}{llll}
u_{i}=\bar{u}_{i} & \text { em } & \Gamma_{u} & i=1,2,3 \\
p_{i}=\bar{p}_{i} & \text { em } & \Gamma_{p} & i=1,2,3
\end{array}
$$

onde $\Gamma=\Gamma_{u}+\Gamma_{p}$. 


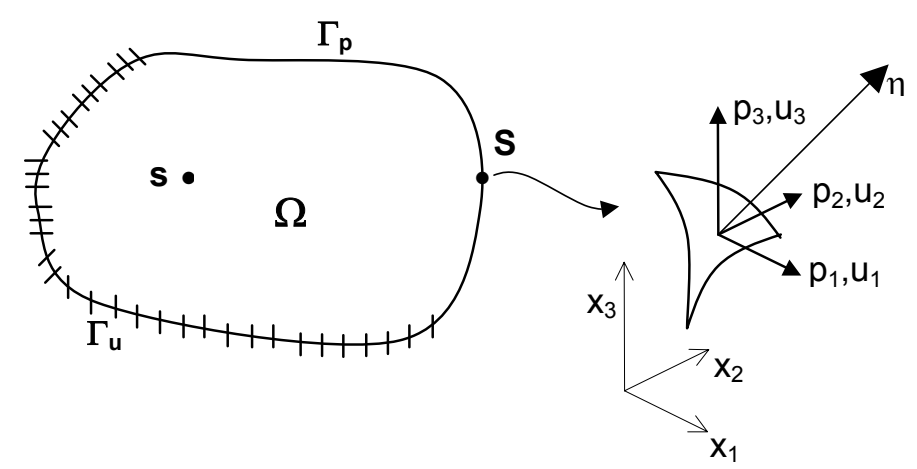

Figura 2.1 - Representação de um sólido com condições de contorno mistas.

\subsection{Representação das equações de equilíbrio no contorno}

As representações das equações de Navier em forma de equações integrais podem ser obtidas com a utilização das técnicas dos resíduos ponderados ou, como será aplicado neste texto, pelo $2^{\circ}$ Teorema da Reciprocidade de Betti (TRB).

Pelo TRB, pode-se expressar a relação de igualdade entre dois campos distintos de tensões mediante a aplicação do princípio dos trabalhos virtuais. Seja então um campo representado pelo estado real de deslocamentos, deformações e tensões que se desenvolvem em um corpo elástico isótropo de domínio $\Omega$ e contorno $\Gamma$, sob a ação de trabalho externo.

Considere-se, também, um estado de forças fictícias atuando neste mesmo corpo em um ponto genérico " $p$ " do domínio. Os campos virtuais que se desenvolvem em função deste estado virtual são os deslocamentos, as deformações e as tensões virtuais que também devem atender as relações (2.2) e (2.7).

Tem-se, desta forma, as variáveis relativas ao problema real, ou seja: $\sigma_{i j}(s), u_{i}(s), \varepsilon_{i j}(s)$ para os campos desenvolvidos no ponto "s"; e as forças atuantes $b_{i}(s), p_{i}(S)$. E para um estado virtual, indicado com o sobrescrito "*”, os campos desenvolvidos devidos a uma força unitária aplicada na direção "k" são: $\sigma_{i j}^{*}(p, s), u_{i}^{*}(p, s), \varepsilon_{i j}^{*}(p, s)$. 
Esses dois estados podem ser relacionados fazendo-se uso da relação (2.7), que é reescrita abaixo:

$$
\sigma_{i j}(s)=c_{i j k l} \varepsilon_{k l} \quad i, j, k, l=1,2,3
$$

e para o estado virtual:

$$
\sigma^{*}{ }_{i j}(s)=c_{i j k l} \varepsilon^{*}{ }_{k l} \quad i, j, k, l=1,2,3
$$

de modo que eles podem ser relacionados como:

$$
\sigma_{i j}(s) \cdot \varepsilon_{i j}^{*}=c_{i j k l} \cdot \varepsilon_{k l} \cdot \varepsilon^{*}{ }_{i j}=\varepsilon_{k l} \cdot\left(c_{i j k l} \cdot \varepsilon^{*} i j\right) \quad i, j, k, l=1,2,3
$$

e sabendo-se que o tensor constitutivo é simétrico, é possível se escrever:

$$
\int_{\Omega} \sigma_{i j}^{*} \cdot \varepsilon_{i j} \partial \Omega=\int_{\Omega} \varepsilon_{i j}^{*} \cdot \sigma_{i j} d \Omega \quad i, j=1,2,3
$$

Integrando-se por partes ambos os lado da expressão (2.15), e levando-se em conta as relações (2.2), (2.4) e (2.6), chega-se ao $2^{\circ}$ Teorema da Reciprocidade de Betti, que relaciona dois diferentes problemas elásticos estabelecidos dentro do mesmo domínio:

$$
\int_{\Omega} b_{k}^{*} \cdot u_{k} d \Omega+\int_{\Omega} p_{k}^{*} \cdot u_{k} d \Gamma=\int_{\Omega} b_{k} \cdot u_{k}^{*} d \Omega+\int_{\Omega} p_{k} \cdot u_{k}^{*} d \Gamma \quad k=1,2,3
$$

As forças de volume do estado virtual podem ser associadas a cargas unitárias concentradas aplicadas no ponto "p" $\in \Omega^{*}$ em cada uma das três direções ortogonais, e são definidas como uma função Delta de Dirac de modo que se tem:

$$
b_{k}^{*}=\delta(p, s) \cdot \delta_{k i} \quad k, i=1,2,3
$$

onde $\delta_{\mathrm{ij}}$ é a função Delta de Kronecker, e $\delta(p, s)$ é a função de Dirac, que por definição tem as seguintes propriedades: 


$$
\begin{aligned}
& \delta(p, s)=0, \text { se } p \neq s ; \\
& \delta(p, s)=\infty, \text { se } p=s ; \quad e \\
& \int_{\Omega} \delta(p, s) \cdot f(s) d \Omega=f(p)
\end{aligned}
$$

Aplicando a expressão (2.17) na primeira integral de (2.16), e definindo $u_{k i}^{*}(p, s)$ e $p_{k i}^{*}(p, s)$ como os deslocamentos e forças de superfície na direção $j$ no ponto "p", devido à uma força unitária aplicada na direção $i$ no ponto "s", chega-se a:

$$
u_{k}(p)+\int_{\Gamma} p_{k i}^{*}(p, S) \cdot u_{i}(S) d \Gamma(S)=\int_{\Gamma} u_{k i}^{*}(p, S) \cdot p_{i}(S) d \Gamma(S)+\int_{\Omega} u_{k i}^{*}(p, s) \cdot b_{i}(s) d \Omega(s) k, i=1,2,3
$$

Essa relação é conhecida como Identidade Somigliana e é a representação integral de deslocamentos dos pontos internos ao sólido em termos de valores de contorno.

A relação (2.19) é a solução da equação de Navier (2.7), onde se adotou aplicar a força unitária em um ponto "S" no contorno do domínio $\Omega$ ou fora deste. Entretanto, os valores do contorno não podem ser obtidos ainda, uma vez que o primeiro termo da equação (2.19) está associado a valores internos ao domínio $\Omega$.

Assim, a estratégia que se adota para representar as equações integrais com valores apenas associados ao contorno "S", consiste em levar " $p$ " - que está no interior do corpo - para uma região no contorno "P". Isto é feito somando-se um domínio infinitesimal de modo que o ponto de contorno "P" fique sendo um ponto de domínio (vide figura 2.2). Com a introdução deste novo domínio $-\left(\Omega+\Omega_{\varepsilon}\right)-\mathrm{e}$ deste novo contorno infinitesimal $-\left(\Gamma-\bar{\Gamma}+\Gamma_{\varepsilon}\right)-$ a Identidade Somigliana, equação (2.19), é reescrita como:

$$
\begin{aligned}
& u_{k}(p)+\ell i m_{\varepsilon \rightarrow 0}\left[\int_{\Gamma-\Gamma+\Gamma_{\varepsilon}} p_{k i}^{*}(P, S) \cdot u_{i}(S) d \Gamma(S)\right]=\ell m_{\varepsilon \rightarrow 0}\left[\int_{\Gamma-\bar{\Gamma}+\Gamma_{\varepsilon}} u_{k i}^{*}(P, S) \cdot p_{i}(S) d \Gamma(S)+\right. \\
& \left.\int_{\Omega+\Omega_{\varepsilon}} u_{k i}^{*}(P, s) \cdot b_{i}(s) d \Omega(s)\right]
\end{aligned}
$$




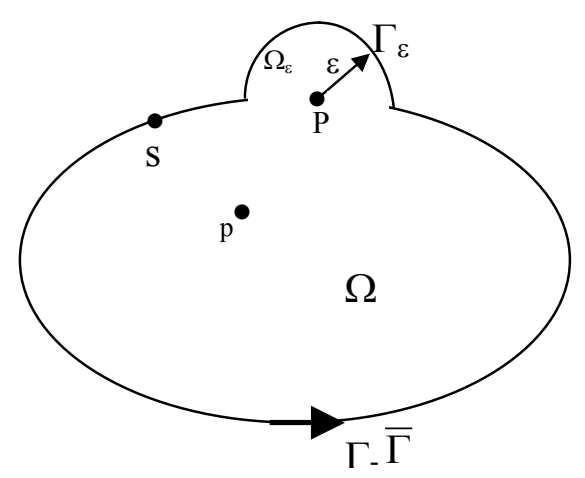

Figura 2.2 - Domínio acrescido de complemento infinitesimal para o caso 3D.

quando $\varepsilon \rightarrow 0$, a parcela abaixo tende a zero, ou seja:

$$
\lim _{\varepsilon \rightarrow 0}\left[\int_{\Gamma_{\varepsilon}} u_{k i}^{*}(P, S) \cdot p_{i}(S) d \Gamma(S)\right]=0 \quad k, i=1,2,3
$$

enquanto que

$$
\lim _{\varepsilon \rightarrow 0}\left[\int_{\Gamma-\Gamma} u_{k i}^{*}(P, S) \cdot p_{i}(S) \partial \Gamma(S)\right]=\int_{\Gamma} u_{k i}^{*}(P, S) \cdot p_{i}(S) \partial \Gamma(S) \quad k, i=1,2,3
$$

e a parcela

$$
\left.\lim _{\varepsilon \rightarrow 0}\left[\int_{\Gamma-\Gamma} p_{k i}^{*}(P, S) \cdot u_{i}(S) d \Gamma(S)\right]=\int_{\Gamma} p_{k i}^{*}(P, S) \cdot u_{i}(S) d \Gamma(S)\right] \quad k, i=1,2,3
$$

apresenta uma singularidade que é interpretada no sentido de valor principal de Cauchy, e deve ser tratada cuidadosamente para cada solução fundamental.

O último termo da integral de contorno é dado por:

$$
\int_{\Gamma_{\varepsilon}} p_{k i}^{*}(P, S) \cdot u_{i}(S) d \Gamma(S)=C_{k i}(P) \cdot u_{i}(P) \quad k, i=1,2,3
$$

onde $C_{k i}$ são coeficientes de uma matriz, denominados termos livre de integração, os quais dependem da geometria do contorno (vide HARTMANN (1980)). 
Desse modo, chega-se a representação da equação de equilíbrio no contorno que é escrita como:

$C_{k i}(P) \cdot u_{k}(P)+\int_{\Gamma} p_{k i}^{*}(P, S) \cdot u_{i}(S) d \Gamma(S)=\int_{\Gamma} u_{k i}^{*}(P, S) \cdot p_{i}(S) d \Gamma(S)+\int_{\Omega} u_{k i}^{*}(P, s) \cdot b_{i}(s) d \Omega(s)$

\subsection{A solução fundamental tridimensional de Kelvin}

A solução de Kelvin (LOVE (1944)), corresponde à obtenção das soluções das equações de equilíbrio (2.7) para um corpo sólido esférico de raio infinito para a aplicação de um conjugado de forças ortogonais e pontuais dentro do corpo, com a condição de que os deslocamentos tendam a zero em distâncias infinitas da ordem de pelo menos $r^{-2}$. As perturbações locais que aparecem na vizinhança do ponto de aplicação levam a singularidades nas soluções fundamentais, tendo-se que ser tratadas adequadamente. Entretanto, pelo princípio de Saint-Venant, as distâncias suficientemente afastadas do ponto de aplicação da força causam os deslocamentos e as tensões independentemente da forma de sua aplicação.

Assim, a solução fundamental de Kelvin é a formulação mais geral para um corpo homogêneo e isótropo de geometria arbitrária, sendo que os campos mobilizados de deslocamentos e tensões que ocorrem em um ponto "S" - ponto campo - devido a uma força unitária aplicada em "P" - ponto fonte - são dados, respectivamente, por:

$$
\begin{gathered}
u_{k i}^{*}(P, S)=\frac{1}{16 \pi G(1-v) \cdot r}\left[(3-4 v) \cdot \delta_{k i}+r_{, k} \cdot r_{, i}\right] \\
p_{k i}^{*}(P, S)=\frac{-1}{8 \pi(1-v) \cdot r^{2}}\left\{r_{, n}\left[(1-2 v) \cdot \delta_{k i}+3 r_{, k} \cdot r_{, i}\right]+(1-2 v)\left(\eta_{k} r_{, i}-\eta_{i} r_{, k}\right)\right\}
\end{gathered}
$$

onde " $k$ " é a direção de aplicação da força e "i" é a direção do campo mobilizado, variando de1 a 3 para o caso tridimensional, e $r=|X(P)-X(S)|$

$r_{, i}=\frac{r_{i}}{r}$ 
$r_{, n}=\frac{\partial r}{\partial x_{i}} \cdot \frac{\partial x_{i}}{\partial \eta}=\frac{r_{i}}{r} \cdot \eta_{i}$

e $\eta_{k}$ é o co-seno diretor da normal ao contorno "S" em relação à direção $x_{k}$.

\subsubsection{Termo livre e pontos no domínio}

O termo, representado na expressão (2.24), corresponde a uma parcela obtida pelo cálculo de uma das integrais de superfície sobre uma região esférica, e seus valores dependem da geometria do contorno do ponto "P" em questão.

Em HARTMANN (1980), é apresentado o procedimento usado para se obter esta integral. Assim, reescrevendo-se a relação (2.24):

$$
\int_{\Gamma_{\varepsilon}} p_{k i}^{*}(P, S) \cdot u_{i}(S) d \Gamma(S)=C_{k i}(P) \cdot u_{i}(P) \quad k, i=1,2,3
$$

onde $\mathrm{C}_{\mathrm{ki}}$, para o caso tridimensional, é uma matriz $(3 \times 3)$ que depende da posição do ponto "P" e de seu octante. Em relação à posição desse ponto, três situações distintas podem surgir:

$$
C_{k i}(P)=\left\{\begin{array}{lll}
I & \text { se } & P \in \Omega \\
C_{k i}(P) & \text { se } \quad P \in \Gamma \\
0 & \text { se } & P \text { fora do corpo }
\end{array}\right.
$$

onde $I$ é a matriz identidade e 0 é a matriz nula.

Os valores da matriz de termos livres, quando o ponto "P" está sobre o contorno, podem ser determinados pelo cálculo da integral da expressão apresentada em HARTMANN (1980, pg. 374). No caso de os vértices serem ortogonais, os coeficientes da matriz C podem ser de três tipos:

a) Metade de uma esfera (contorno suave): 


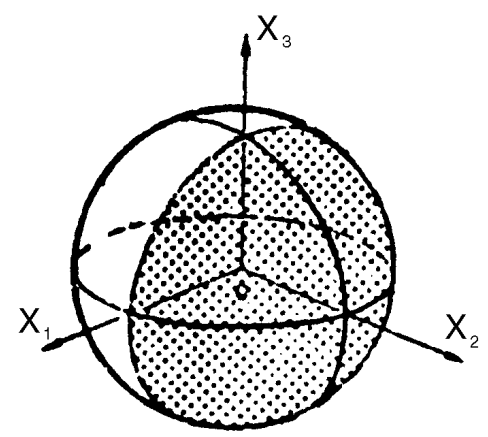

$$
C_{k i}(P)=\frac{1}{8 \cdot \pi} \cdot\left[\begin{array}{ccc}
4 \cdot \pi & 0 & 0 \\
0 & 4 \cdot \pi & 0 \\
0 & 0 & 4 \cdot \pi
\end{array}\right]
$$

b) Quarto de esfera (borda):

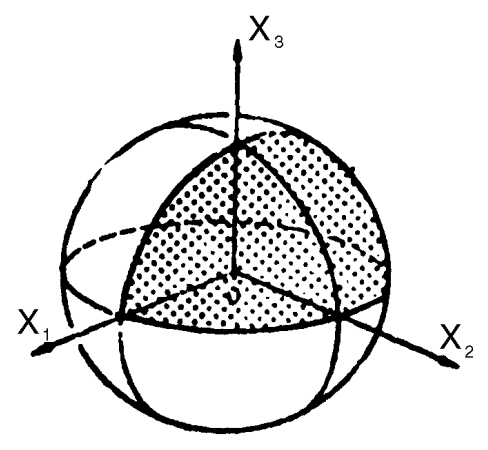

$$
C_{k i}(P)=\frac{1}{8 \cdot \pi} \cdot\left[\begin{array}{ccc}
2 \cdot \pi & 0 & 0 \\
0 & 2 \cdot \pi & \frac{2}{1-v} \\
0 & \frac{2}{1-v} & 2 \cdot \pi
\end{array}\right]
$$

c) Oitavo de esfera (canto):

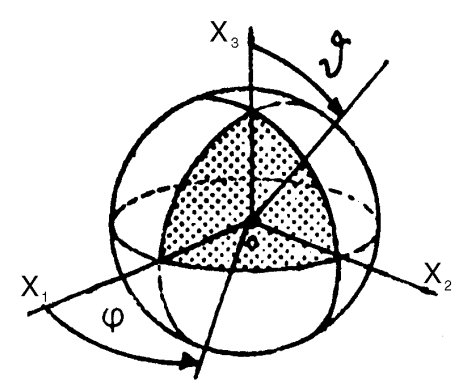

$$
C_{k i}(P)=\frac{1}{8 \cdot \pi} \cdot\left[\begin{array}{ccc}
\pi & \frac{1}{1-v} & \frac{1}{1-v} \\
\frac{1}{1-v} & \pi & \frac{1}{1-v} \\
\frac{1}{1-v} & \frac{1}{1-v} & \pi
\end{array}\right]
$$

Figura 2.3 - Valores dos termos da matriz C para três casos de cantos ortogonais.

Os valores apresentados da matriz C são válidos para uma posição do vértice no primeiro octante da esfera, e para os outros octantes, os termos das matrizes apresentadas nos três casos anteriores serão alterados em sinais conforme as normais de seu octante. Uma forma de se determinar os sinais corretos dos termos é alterar na expressão de HARTMANN (1980, pg. 374) os sinais dos valores trigonométricos conforme os ângulos esféricos determinados pela posição de seu octante. 
Após se conhecer os valores de contorno, os deslocamentos de domínio podem ser calculados aplicando-se diretamente a Identidade Somigliana (2.19), e, para o cálculo das tensões no domínio do corpo, é necessário diferenciar a equação (2.19), e para um ponto " $s$ " do domínio tem-se:

$$
\sigma_{i j}(s)=-\int_{\Gamma} S_{k j}(p, S) \cdot u_{k}(S) d \Gamma+\int_{\Gamma} D_{k i j}(p, S) \cdot p_{k}(S) d \Gamma \quad i, j, k=1,2,3
$$

sendo os tensores $D_{k i j}$ e $S_{k i j}$ para o caso 3D, respectivamente dados por:

$$
\begin{gathered}
D_{k i j}=\frac{1}{8 \pi(1-v) r^{2}}\left\{(1-2 v) \cdot\left[\delta_{k i} r_{, j}+\delta_{k j} r_{, i}-\delta_{i j} r_{, k}\right]+3 r_{, k} r_{, i} r_{, j}\right\} \quad i, j, k=1,2,3 \\
S_{k i j}=\frac{G}{4 \pi(1-v) r^{3}}\left\{3 r_{, n}\left[(1-2 v) \delta_{i j} r_{, k}+v\left(\delta_{i k} r_{, j}+\delta_{j k} r_{, i}\right)-5 r_{, k} r_{,} r_{, j}\right]+\right. \\
\left.3 v\left(\eta_{i} r_{, j} r_{, k}+\eta_{j} r_{, i} r_{, k}\right)+(1-2 v)\left(3 \eta_{k} r_{, i} r_{, j}+\eta_{j} \delta_{i k}+\eta_{i} \delta_{j k}\right)-(1-4 v) \eta_{k} \delta_{i j}\right\} \quad i, j, k=1,2,3
\end{gathered}
$$

A equação (2.29) para obtenção das tensões no domínio não apresenta termos singulares, entretanto para pontos muitos próximos do contorno - ou no caso de se querer calcular as tensões de contato entre dois meios diferentes - em virtude da grande proximidade entre o ponto de domínio "s" e o de contorno "S", os tensores indicados nas relações (2.30) e (2.31) possuem núcleos da ordem de $1 / r^{2}$ e $1 / r^{3}$, sendo necessário empregar integrações numéricas especiais para se obter respostas precisas de tensões. E, para se obter os campos de deslocamentos do domínio emprega-se a relação (2.19).

\subsection{Método dos elementos de contorno}

Para a determinação do campo de deslocamentos e de tensões em um corpo sob condições de contorno e forças aplicadas genéricas, em geral, a solução analítica destas equações diferenciais parciais não é possível. Considerase, então, campos discretos no contorno associados a parâmetros nodais que representem funções aproximadas dos campos de deslocamentos e de forças de 
superfície. Estes campos discretos serão indicados por elementos que devem moldar o contorno do corpo.

Desta maneira, com os campos aproximados do contorno, é possível representar as equações integrais de contorno em equações algébricas escritas em termos dos parâmetros discretos de deslocamentos e de forças de superfície.

Assim, o Método dos Elementos de Contorno (MEC) se baseia na montagem de um sistema algébrico advindo da determinação das equações integrais de contorno da relação (2.25), escritas em termos de parâmetros discretos, tanto de deslocamentos como de forças de superfície. Destaca-se que, para a montagem deste sistema algébrico, é necessário aplicar o mesmo número de estados fundamentais (virtuais) que o necessário para obter os estados reais aproximados, sendo que todo o contorno é representado por elementos, assim para o caso 3D, os elementos têm que ser de geometria de superfície (2D).

\subsubsection{Elemento de superfície}

Nesse trabalho foi adotado o elemento de superfície triangular plano. Este elemento já é bem conhecido na literatura, principalmente aplicado à resolução de problemas planos pelo método dos elementos finitos (ver SAVASSI (1996)).

As variáveis nodais de contorno estão localizadas nos vértices do elemento e para se conhecer a posição e os co-senos diretores deste elemento, basta identificar as coordenadas cartesianas dos vértices no espaço 3D. Adotando-se um dos vértices como uma origem cartesiana local $\left(O^{\prime}\right)$ e um lado deste elemento que passe por esta origem como um eixo local, é possível - mediante produtos vetoriais entre este vetor eixo e seus eixos perpendiculares - calcular estes cosenos direcionais (ver detalhes em Onãte (1995)). 


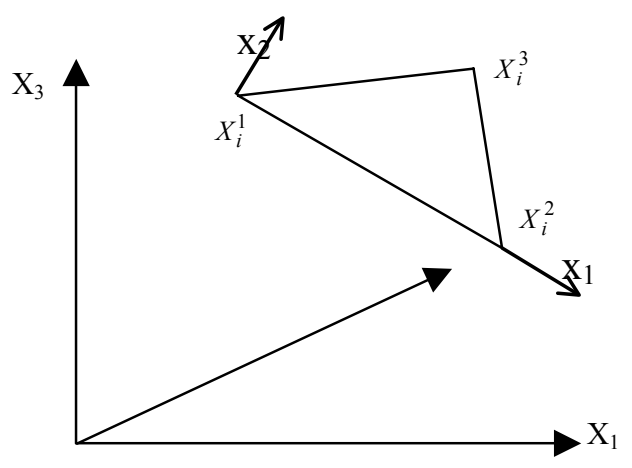

a )

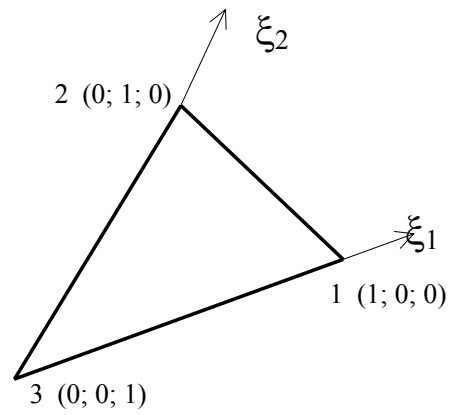

b )

Figura 2.4 - a) Geometria do elemento e sistemas de coordenadas; b) Coordenadas oblíquas.

Além das coordenadas cartesianas (figura 2.4a), costuma-se utilizar também um sistema homogêneo ou oblíquo que facilita o cálculo das integrais, pois assim os limites de integração ficam os mesmos, alterando apenas o Jacobiano de cada elemento. A localização deste sistema oblíquo no plano do elemento pode ser visualizado na figura $2.4 \mathrm{~b}$. Sendo assim, a posição (S) sobre o elemento é correlacionada, pelas suas coordenadas nodais, à ponderação das funções interpoladoras $\Psi_{i}$. Ou seja:

$$
X_{i}(S)=X_{i}^{1} \cdot \Psi_{1}\left(\xi_{1}, \xi_{2}\right)+X_{i}^{2} \cdot \Psi_{2}\left(\xi_{1}, \xi_{2}\right)+X_{i}^{3} \cdot \Psi_{3}\left(\xi_{1}, \xi_{2}\right)
$$
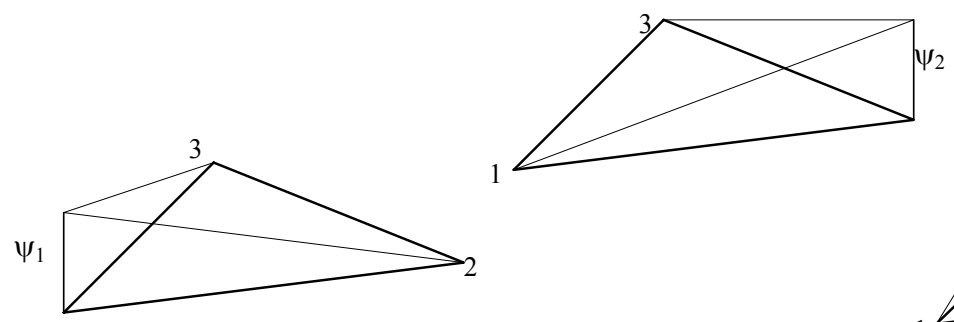

Figura 2.5 - Funções interpoladoras adotadas.

Como a variação adotada para a função interpoladora é linear (figura 2.5), pode-se calcular esta função em coordenadas homogêneas por:

$$
\Psi_{i}\left(\xi_{1}, \xi_{2}\right)=\alpha \xi_{1}+\beta \xi_{2}+\gamma
$$


impondo as condições de contorno de modo que $\Psi_{1}(1,0)=1, \Psi_{1}(0,1)=0$ e $\Psi_{1}(0,0)=0$

$$
\Psi_{1}\left(\xi_{1}, \xi_{2}\right)=\xi_{1}
$$

$\Psi_{2}(1,0)=0, \Psi_{2}(0,1)=1 e \Psi_{2}(0,0)=0$

$$
\Psi_{2}\left(\xi_{1}, \xi_{2}\right)=\xi_{2}
$$

$\Psi_{3}(1,0)=0, \Psi_{3}(0,1)=0$ e $\Psi_{3}(0,0)=1$

$$
\Psi_{3}\left(\xi_{2}, \xi_{3}\right)=\xi_{3}
$$

Assim, tendo-se em vista (2.34), (2.35) e (2.36), a função ponderadora é dada por:

$$
\Psi_{i}\left(\xi_{2}, \xi_{3}\right)=\xi_{i} \quad i=1,2,3
$$

e a relação (2.32) pode ser representada matricialmente por:

$$
\left\{\begin{array}{l}
X_{1}(S) \\
X_{2}(S) \\
X_{3}(S)
\end{array}\right\}=\left[\begin{array}{ccccccccc}
\xi_{1} & \xi_{2} & \xi_{3} & 0 & 0 & 0 & 0 & 0 & 0 \\
0 & 0 & 0 & \xi_{1} & \xi_{2} & \xi_{3} & 0 & 0 & 0 \\
0 & 0 & 0 & 0 & 0 & 0 & \xi_{1} & \xi_{2} & \xi_{3}
\end{array}\right] \cdot\left\{\begin{array}{c}
X_{1}^{1} \\
X_{1}^{2} \\
X_{1}^{3} \\
X_{2}^{1} \\
X_{2}^{2} \\
X_{2}^{3} \\
X_{3}^{1} \\
X_{3}^{2} \\
X_{3}^{3}
\end{array}\right\}
$$

ou

$$
\left\{X_{i}(S)\right\}=\left[\Psi\left(\xi_{n}\right)\right]^{T} \cdot\left\{X_{i}^{n}\right\} \quad i, n=1,2,3
$$

onde $n$ é o vértice do elemento triangular.

As aproximações dos parâmetros de contorno para os deslocamentos e para as forças de superfícies são também aproximadas nesse sistema homogêneo de referência, ou seja: 


$$
\begin{aligned}
& \left\{U_{i}(S)\right\}=\left[\Psi\left(\xi_{n}\right)\right]^{T} \cdot\left\{U_{i}^{n}\right\} \quad i, n=1,2,3 \\
& \left\{P_{i}(S)\right\}=\left[\Psi\left(\xi_{n}\right)\right]^{T} \cdot\left\{P_{i}^{n}\right\} \quad i, n=1,2,3
\end{aligned}
$$

\subsubsection{Representação algébrica das equações integrais de contorno}

Os campos de deslocamentos e de forças de superfície expressos pelas relações $(2.40)$ e $(2.41)$, respectivamente, são obtidos para cada elemento do contorno $\Gamma$, e podem ser escritos da seguinte forma:

$$
\begin{aligned}
& \left\{U_{i}(S)\right\}=\left[\Psi\left(\xi_{n}\right)\right]^{T} \cdot\left\{U_{i}^{n}\right\}^{j} \quad i=1,2,3 ; j=1, \ldots, n e \\
& \left\{P_{i}(S)\right\}=\left[\Psi\left(\xi_{n}\right)\right]^{T} \cdot\left\{P_{i}^{n}\right\}^{j} \quad i=1,2,3 ; j=1, \ldots, n e
\end{aligned}
$$

onde ne é o número de elementos do contorno.

do contorno $\Gamma$ e $|J|$ o jacobiano da transformação de cada elemento.

As equações integrais de (2.25) podem ser escritas pela forma discreta sem a consideração de força de domínio, levando-se em consideração as relações (2.32) e (2.33), da seguinte maneira:

$$
\begin{aligned}
& C_{k i}(P) \cdot u_{k}(P)+\sum_{j=1}^{n e}|J|_{j} \cdot \int_{\Gamma} p_{k i}^{*}(P, S) \cdot \Psi(S) d \xi(S) \cdot\left(U_{i}\right)^{j}= \\
& \sum_{j=1}^{n e}|J|_{j} \cdot \int_{\Gamma} u_{k i}^{*}(P, S) \cdot \Psi(S) d \xi(S) \cdot\left(P_{i}\right)^{j} \quad i, k=1,2,3
\end{aligned}
$$

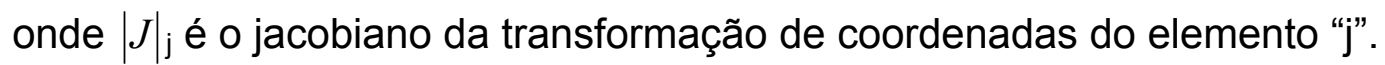

Pode-se, então, montar um sistema matricial, de forma que a expressão (2.44) fique do tipo:

$$
H \cdot U=G \cdot P
$$

onde

$$
H=\bar{H}+\widehat{H}
$$


sendo $\bar{H}$ a matriz diagonal formada pela matriz dos termos livres - C - conferida nas respectivas posições de cada elemento. Se o ponto de origem "P" estiver fora do contorno, então esta matriz $(\bar{H})$ é nula, em função das propriedades da matriz C.

As matrizes $\hat{H}$ e $\mathrm{G}$ são dadas por:

$$
\begin{gathered}
\hat{H}=\sum_{j=1}^{n e}|J|_{j} \cdot \int_{\Gamma} p_{k i}^{*}(P, S) \cdot \Psi(S) d \xi(S) \quad i, k=1,2,3 \\
G=\sum_{j=1}^{n e}|J|_{j} \cdot \int_{\Gamma} u_{k i}^{*}(P, S) \cdot \Psi(S) d \xi(S) \quad i, k=1,2,3
\end{gathered}
$$

Lembrando-se que, para cada ponto de origem "P" no contorno, o elemento a ser integrado que contenha este ponto "P", na parcela da integral de $\hat{H}$, possui uma singularidade forte que deve ser tratada como valor principal de Cauchy.

As condições de contorno para cada problema devem contemplar, obrigatoriamente, para cada ponto nodal, as condições de fronteira indicadas nas relações (2.9), (2.10), (2.11) ou (2.12). Então, metade das incógnitas da expressão (2.44) são conhecidas. Portanto, é possível reagrupá-las da seguinte maneira:

$$
A \cdot X=B
$$

onde a matriz $\mathrm{A}$ contém valores referentes às influências tanto das forças de superfície quanto às parcelas de deslocamentos, e essa matriz é densa e nãosimétrica. $O$ vetor $X$ contém os valores incógnitos de cada ponto nodal ou de deslocamento ou de força de superfície, e o vetor $B$ possui os valores prescritos já multiplicados pelas suas correspondentes parcelas integrais.

Finalmente, com os valores de contorno conhecidos, pode se obter os valores internos das tensões mediante a utilização das equações (2.19) e (2.29).

\subsubsection{Integração numérica e subelementação}

A integração numérica com o ponto externo ao domínio é um procedimento alternativo empregado para se evitar a realização das integrais analíticas. Além do 
fato de não se ter que calcular a parcela do termo livre, principalmente para o problema 3D, onde para o caso geral se requer um trabalho não tão imediato.

Assim sendo, as parcelas das integrais de contorno, que são expressas pelas relações (2.48) e (2.49), ficam representadas pelas coordenadas dos pontos de integração: $\xi_{i}^{j}$ e de fatores de peso - $w_{j}$ - associados a esta posição, e em BREBBIA \& DOMINGUEZ (1989) são tabelados estes pontos para as coordenadas oblíquas. Ou seja, as parcelas da matriz h e g ficam para cada elemento:

$$
\begin{aligned}
& \widehat{H}=\sum_{j=1}^{n e} \sum_{m=1}^{n}|J|_{j} \cdot w_{m} \cdot\left[p_{k i}^{*}\left(P, S^{m}\right) \cdot \Psi\left(\xi_{1}^{m}, \xi_{2}^{m}, \xi_{3}^{m}\right)\right] \quad i, k=1,2,3 \\
& G=\sum_{j=1}^{n e} \sum_{m=1}^{n}|J|_{j} \cdot w_{m} \cdot\left[u_{k i}^{*}\left(P, S^{m}\right) \cdot \Psi\left(\xi_{1}^{m}, \xi_{2}^{m}, \xi_{3}^{m}\right)\right] \quad i, k=1,2,3
\end{aligned}
$$

onde $n$ é o número de pontos de Hammer, $S^{m}$ é a posição do ponto de integração que representa o ponto campo para o cálculo da influência da solução fundamental.

Quando o ponto fonte está muito perto ao elemento a ser integrado, é comum utilizar nesta região uma integração mais refinada. Assim, costuma-se empregar a técnica da subelementação convencional que nada mais é do que dividir estes elementos quase-singulares em outros menores. Isso faz com que seja possível introduzir em cada novo subelemento mais pontos de integração.

O método convencional empregado é a divisão de forma regular dentro do elemento (ver figura 2.6). Para este caso, os novos elementos criados não apresentarão novos parâmetros, mas seus pontos de integração serão associados às variáveis nodais dos vértices. Ou seja, reescrevendo-se a relação (2.39):

$$
\left\{X_{i}(S)\right\}=\left[\Psi\left(\xi_{n}\right)\right]^{T} \cdot\left\{X_{i}^{n}\right\} \quad i, n=1,2,3
$$




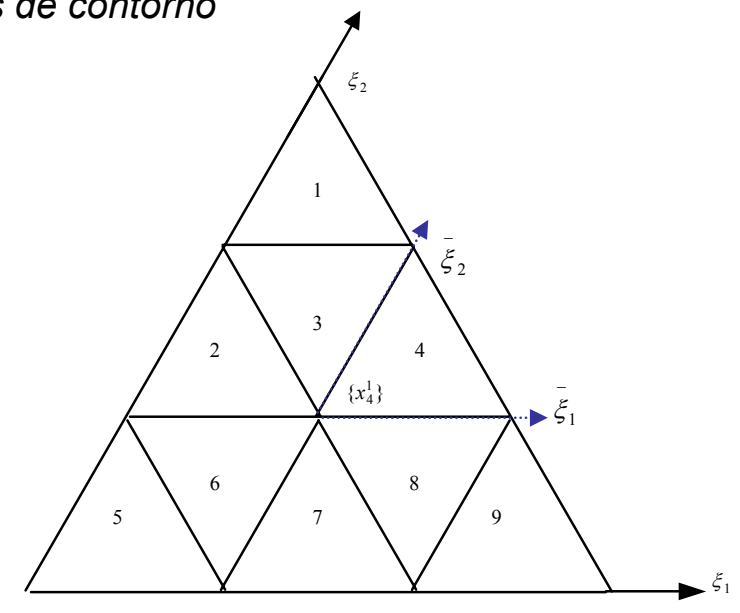

Figura 2.6 - Subelementação regular e coordenadas locais e globais.

i)
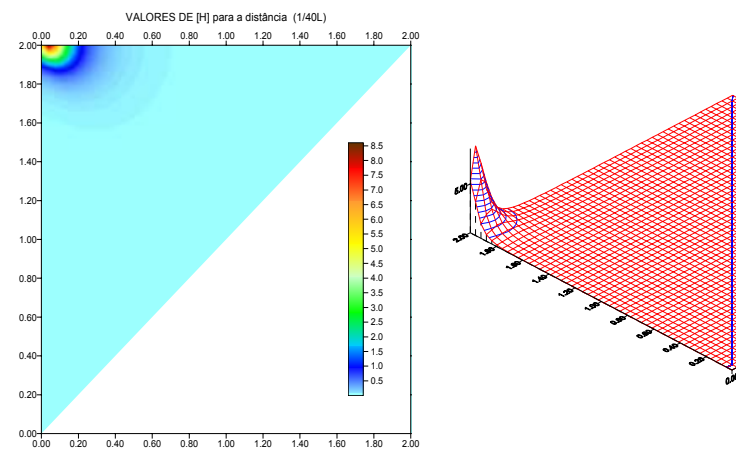

ii)
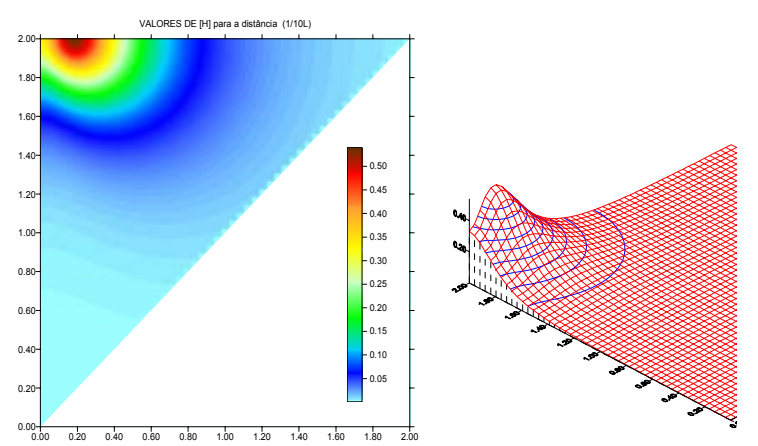

iii)
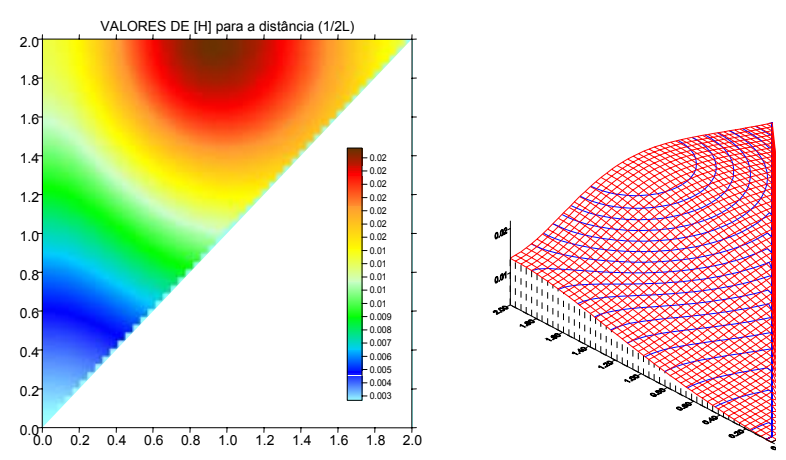

Figura 2.7 - Gráficos das integrais no elemento quase-singular; i) ponto fora a 1/40L ii) ponto fora a $1 / 10 L$; iii) ponto fora a $1 / 2 L$ ( $L$ é maior lado). 
pode-se, então, escrever para uma mudança de sistema:

$$
\left\{x_{i}(S)\right\}=\left[\bar{\Psi}\left(\bar{\xi}_{n}\right)\right]^{T} \cdot\left\{x_{i}^{n}\right\} \quad i, n=1,2,3
$$

onde $x_{i}(S), x_{i}^{n}$ são - respectivamente - as coordenadas e os parâmetros dos vértices de cada subelemento, $\bar{\xi}_{n}$ é o sistema homogêneo posicionado em um sistema oblíquo local do subelemento (vide figura 2.6). Pode-se escrever, então:

$$
\left\{x_{i}^{n}\right\}=\left[\Psi\left(\bar{\xi}_{n}\right)\right]^{T} \cdot\left\{X_{i}^{n}\right\} \quad i, n=1,2,3
$$

Confrontando-se (2.52) com (2.53), chega-se a:

$$
\hat{\Psi}\left(\xi_{n}\right)=\Psi\left(\bar{\xi}_{n}\right) \cdot \bar{\Psi}\left(\bar{\xi}_{n}\right) \quad n=1,2,3
$$

$E$, então

$$
\left\{x_{i}(S)\right\}=\left[\hat{\Psi}\left(\xi_{n}\right)\right] \cdot\left\{X_{i}^{n}\right\} \quad i, n=1,2,3
$$

sendo que a matriz que relaciona as coordenadas homogêneas local com a global do subelemento "k" é dada por

$$
\left[\hat{\Psi}\left(\xi_{n}\right)\right]_{k}=\left\{\begin{array}{l}
\xi_{1} \\
\xi_{2} \\
\xi_{3}
\end{array}\right\}_{k}=\left[\begin{array}{lll}
\xi_{1}^{1} & \xi_{1}^{2} & \xi_{1}^{3} \\
\xi_{2}^{1} & \xi_{2}^{1} & \xi_{2}^{1} \\
\xi_{3}^{1} & \xi_{3}^{1} & \xi_{3}^{1}
\end{array}\right]_{k} \cdot\left\{\begin{array}{l}
\bar{\xi}_{1} \\
\bar{\xi}_{2} \\
\bar{\xi}_{3}
\end{array}\right\}_{k}
$$

Assim, foi desenvolvida uma rotina em que, mediante o número de divisões do elemento quase-singular, é calculado o número de subelementos e as integrais numéricas são realizadas.

Para este caso as parcelas de $\hat{H}$ e $\mathrm{G}$ ficam:

$$
\widehat{H}=\sum_{j=1}^{n e} \sum_{l=1}^{n s} \sum_{m=1}^{n} \frac{|J|_{j}}{n s} \cdot w_{m} \cdot\left[p_{k i}^{*}\left(P, S^{m}\right) \cdot \Psi\left(\xi_{1}^{m}, \xi_{2}^{m}, \xi_{3}^{m}\right)\right] \quad i, k=1,2,3
$$




$$
G=\sum_{j=1}^{n e} \sum_{l=1}^{n s} \sum_{m=1}^{n} \frac{|J|_{j}}{n s} \cdot w_{m} \cdot\left[u_{k i}^{*}\left(P, S^{m}\right) \cdot \Psi\left(\xi_{1}^{m}, \xi_{2}^{m}, \xi_{3}^{m}\right)\right] \quad i, k=1,2,3
$$

onde ns é o número total de subelementos.

Entretanto, este procedimento é muito dispendioso computacionalmente, e, conforme pode ser notado nas figuras (2.7i-iii), para os pontos muito próximos do elemento quase-singular, o comportamento do termo (1/r) do integrando das equações (2.47) e (2.48) são muito dominantes. Assim, o que melhor representaria a subdivisão deste elemento seria aumentar a densidade dos subelementos nesta região diminuindo-a progressivamente ao se distanciar do ponto fonte.

Sob este enfoque, alguns trabalhos já foram desenvolvidos para melhorar esta integração, citando-se, por exemplos, os artigos de HUANG \& CRUSE (1993), LACHAT \& WATSON (1976), MUSTOE (1984), JUN et al. (1985), TELLES (1987) e SOUZA et al. (2002). Onde SOUZA et al. (2002) aplicam a integração numérica unidimensional, via quadratura de Gauss, tanto para o ângulo como para o raio. Sendo que é realizada uma subelementação auto-adaptativa para a integração radial, assim quanto mais longe do ponto fonte, menos elementos e menos pontos de integração se utilizam.

Quanto a esse aspecto, neste presente trabalho também se propõe uma técnica de subelementação progressiva para os elementos triangulares. Entretanto, a técnica aqui proposta subdivide o elemento quase-singular em triângulos menores de forma que se concentrem mais subelementos próximo ao ponto fonte, e, conforme se distancie deste, a densidade da malha vai diminuindo tanto no aumento do tamanho do elemento como também no número de pontos de integração. Na figura 2.8, mostra-se um exemplo de uma densidade adotada bem como dos parâmetros necessários para sua montagem. 


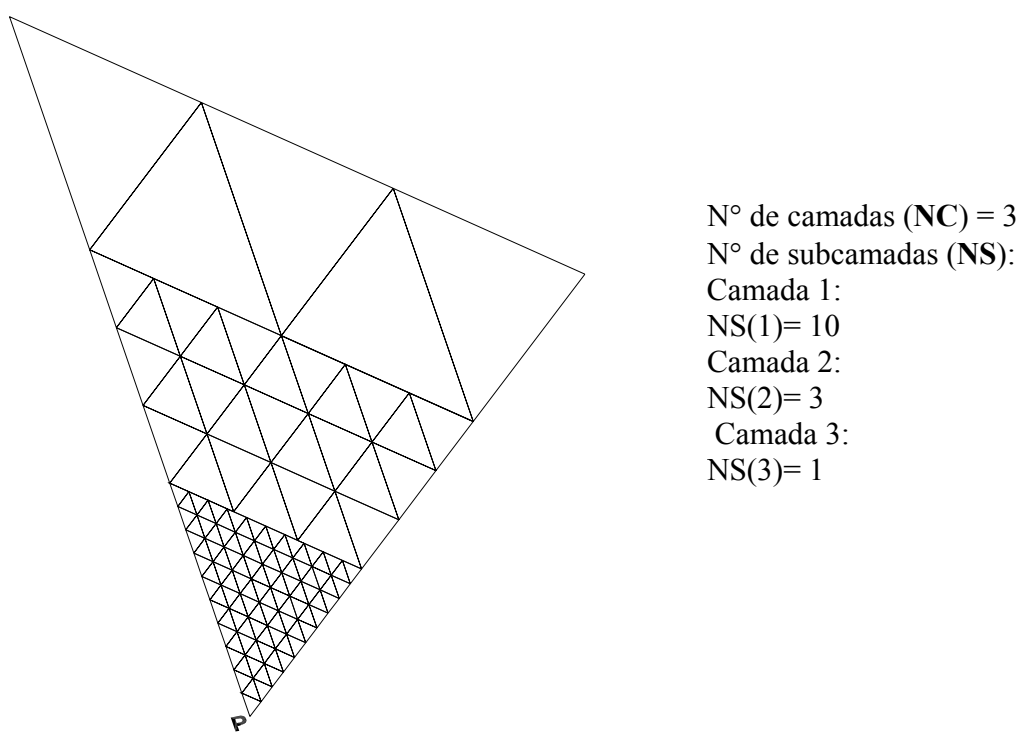

Figura 2.8 - Subelementação progressiva para a posição " $P$ " e parâmetros necessários.

A posição da subelementação mais refinada acompanha a localização do ponto fonte. Desta forma, pode-se mostrar que as parcelas de $\hat{H}$ e $G$ são dadas por:

$$
\begin{aligned}
& \widehat{H}=\sum_{j=1}^{n e} \sum_{l=1}^{N C} \sum_{t=1}^{N S(l)} \frac{|J|_{j}}{(N S(t) \cdot N C)^{2}} \sum_{m=1}^{n} \cdot w_{m} \cdot\left[p_{k i}^{*}\left(P, S^{m}\right) \cdot \Psi\left(\xi_{1}^{m}, \xi_{2}^{m}, \xi_{3}^{m}\right)\right] \quad i, k=1,2,3 \\
& G=\sum_{j=1}^{n e} \sum_{l=1}^{N C} \sum_{t=1}^{N S(l)} \frac{|J|_{j}}{(N S(t) \cdot N C)^{2}} \sum_{m=1}^{n} \cdot w_{m} \cdot\left[u_{k i}^{*}\left(P, S^{m}\right) \cdot \Psi\left(\xi_{1}^{m}, \xi_{2}^{m}, \xi_{3}^{m}\right)\right] \quad i, k=1,2,3 .
\end{aligned}
$$

Onde NC é o número de camadas do elemento e NS(NC) é o vetor que contém o número de subcamadas de cada camada NC.

\subsubsection{Integração analítica}

Para o caso em que o ponto de colocação "P" esteja situado sobre o contorno $\left(\Gamma_{\mathrm{j}}\right)$, as integrais expressas em (2.47) e (2.48) podem ser tratadas semianaliticamente. 
Uma técnica bem utilizada para calcular estas integrais (ver BARBIRATO (1999)) é mediante uma transformação de coordenadas, passando de um sistema cartesiano $(x, y, z)$ para o sistema polar $(r, \theta)$, sendo que a variável $r$ é integrada analiticamente enquanto a variável $\theta$ é feita numericamente. Assim, já escrevendo as relações (2.47) e (2.48) neste novo sistema de coordenadas, levando-se em conta que

$$
d \Gamma=r d r d \theta
$$

tem-se, para um elemento genérico "j"

$$
\begin{aligned}
\hat{H}_{k i}^{j} & =\int_{\Gamma_{j}} \frac{-1}{8 \pi(1-v) \cdot r}(1-2 v)\left(\eta_{k} r_{, i}-\eta_{i} r_{, k}\right) \cdot \xi_{l} d r d \theta \quad k, i=1,2,3 \\
G_{k i}^{j} & =\int_{\Gamma_{j}} \frac{1}{16 \pi G(1-v)}\left[(3-4 v) \cdot \delta_{k i}+r_{, k} \cdot r_{, i}\right] \cdot \xi_{l} d r d \theta \quad k, i=1,2,3
\end{aligned}
$$

As funções ponderadoras escritas em coordenadas homogêneas podem ser associadas às coordenadas cartesianas locais do elemento, conforme SAVASSI (1996), por:

$$
\xi_{l}=\frac{1}{2 \cdot A} \cdot\left[\alpha_{l}+\beta_{l} \cdot x+\gamma_{l} \cdot y\right]
$$

onde

$$
\begin{aligned}
& \alpha_{l}=x_{j} \cdot y_{k}-x_{k} \cdot y_{j} \\
& \beta_{l}=y_{j}-y_{k} \\
& \gamma_{l}=x_{k}-x_{j} \quad(l=1,2,3 \quad j=2,3,1 \quad k=3,1,2)
\end{aligned}
$$

E sabendo-se, como é esquematizado na figura 2.9, que as coordenadas cartesianas podem ser representadas pelas relações polares:

$$
\begin{aligned}
& x=r \cdot \cos \theta \\
& y=r \cdot \operatorname{sen} \theta
\end{aligned}
$$




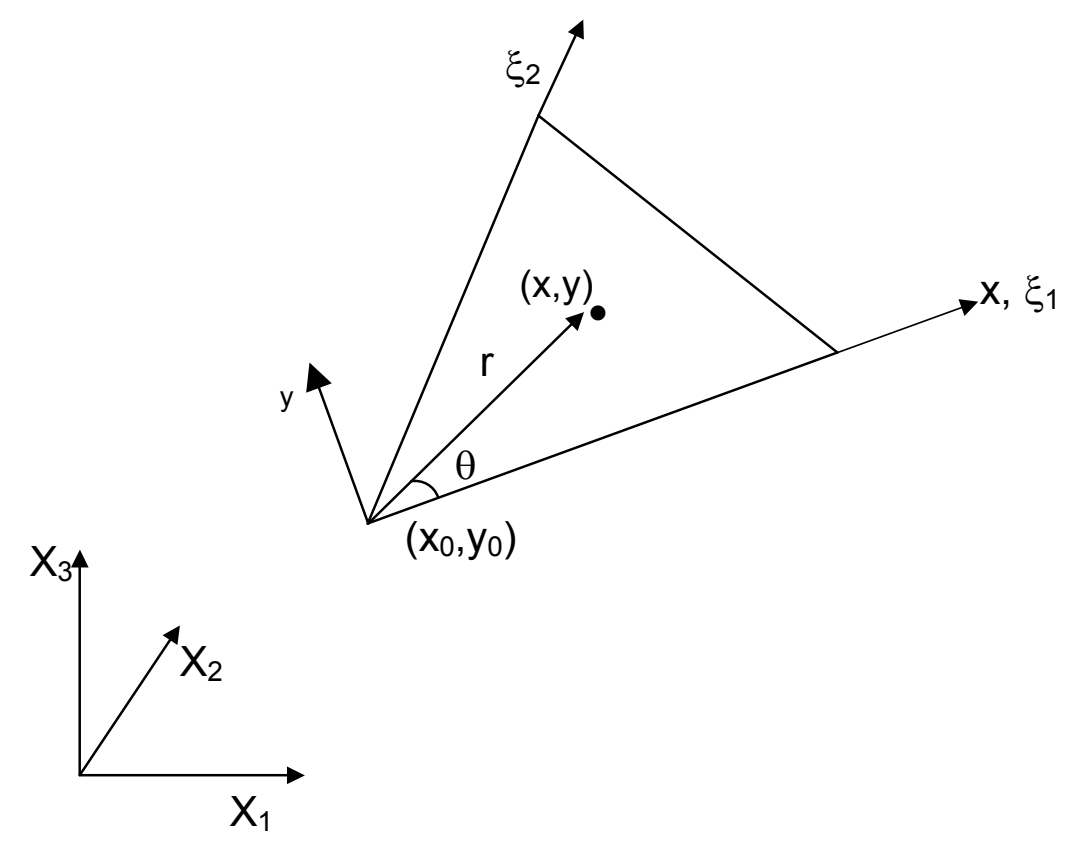

Figura 2.9 - Definição de parâmetros para a integração singular.

Portanto a expressão (2.63) é escrita, já se considerando (2.65) e se realizando as integrais em $r$, assim:

$$
G_{k i}^{j}=\int k_{1}\left[(3-4 v) \cdot \delta_{k i}+r_{, k} \cdot r_{, i}\right] \cdot\left[\alpha_{l}+\frac{\beta_{l}}{2} r^{2} \cdot \cos (\theta)+\frac{\gamma_{l}}{2} r^{2} \cdot \operatorname{sen}(\theta)\right] d r d \theta
$$

Onde

$$
k_{1}=\frac{1}{32 \cdot \pi \cdot G \cdot(1-v) \cdot A}
$$

e A é área do elemento "j".

O termo (2.62) por apresentar a singularidade (1/r) deve ser tratado como valor principal de Cauchy. Para a integração em relação à variável angular $\theta$, realiza-se um procedimento numérico em que se transforma o domínio do contorno $\Gamma_{\mathrm{j}}$ para o contorno do elemento triangular $\hat{\Gamma}_{j}$ (ver figura 2.10).

A transformação do contorno $\Gamma_{\mathrm{j}}$ para o contorno $\hat{\Gamma}_{j}$ é dada pelo jacobiano: 


$$
d \theta=\frac{1}{r} \cdot \frac{d r}{d \hat{n}} d \hat{\Gamma}
$$

Assim, chega-se as relações:

$$
\begin{gathered}
G_{k i}^{j}=\int_{\hat{\Gamma}_{j}} \frac{k_{1}}{2}\left[(3-4 v) \cdot \delta_{k i}+r_{, k} \cdot r_{, i}\right] \cdot\left[2 \alpha_{l}+\beta_{l} \cdot\left(x+x_{0}\right)+\gamma_{l} \cdot\left(y+y_{0}\right)\right] \frac{d r}{d \hat{n}} d \hat{\Gamma} \\
\hat{H_{k i}^{j}}=\int_{\hat{\Gamma}_{j}}-2 \cdot k_{1} \cdot G \cdot\left\{\frac{1}{r} \cdot(1-2 v)\left(\eta_{k} r_{, i}-\eta_{i} r_{, k}\right) \cdot\left[\alpha_{l} \cdot \ln (r)+\beta_{l} \cdot k_{2}+\gamma_{l} \cdot k_{3}\right]\right\} \frac{d r}{d \hat{n}} d \hat{\Gamma}
\end{gathered}
$$

para:

$k_{2}=x+x_{0}[\ln (r)-1]$

$k_{3}=y+y_{0}[\ln (r)-1]$

e onde $\mathrm{x}_{0}$ e $\mathrm{y}_{0}$ representam o ponto de colocação, e a expressão já está em coordenadas globais.

Com as expressões apresentadas anteriormente, pode-se realizar a integração numérica mediante a quadratura de Gauss. Ressalta-se que para o caso do elemento com parâmetros nodais definidos nos vértices do elemento - é o caso deste trabalho -, a parcela $d r / d \hat{n}$ para os lados do elemento que estiverem adjacentes a este vértice é nula. Portanto, realiza-se apenas a integração numérica no elemento oposto ao ponto de colocação. Finalmente, são suficientes poucos pontos de Gauss para a obtenção de resultados precisos desta integração numérica. Neste trabalho adotaram-se 4 pontos de Gauss. 


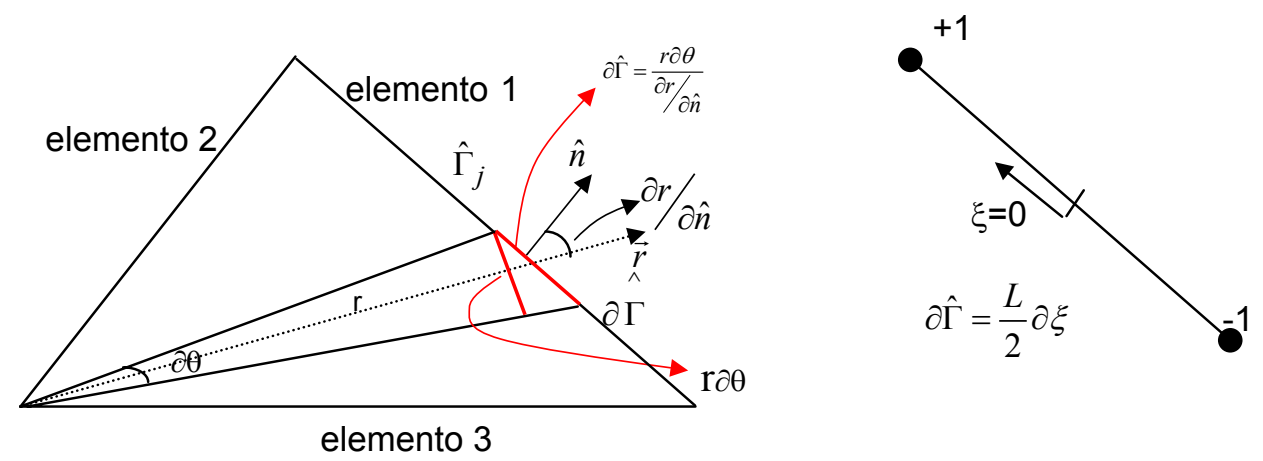

Figura 2.10 - Representação da integração do domínio do elemento pelo seu lados.

\subsection{Sub-região}

Um sólido não-homogêneo pode ser considerado como uma combinação de domínios homogêneos conectados pelos seus contornos em comum (fig. 2.11).

Seja, então, a relação (2.45) aplicada a uma região genérica $m$ de domínio $\Omega_{m}$ e contorno $\Gamma_{m}$

$$
H^{m} \cdot U^{m}=G^{m} \cdot P^{m}
$$

O sólido não-homogêneo é formado por $m$ subdomínios $\left(\Omega=\Omega_{1}+\Omega_{2}+\cdot \cdot+\Omega_{m}\right)$ nos quais a relação (2.70) é válida dentro de seu respectivo domínio.

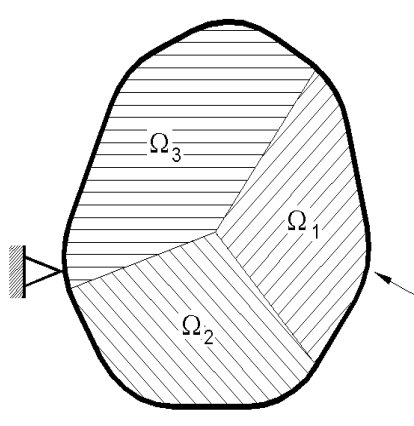

SÓLIDO NÃO-HOMOGÊNEO

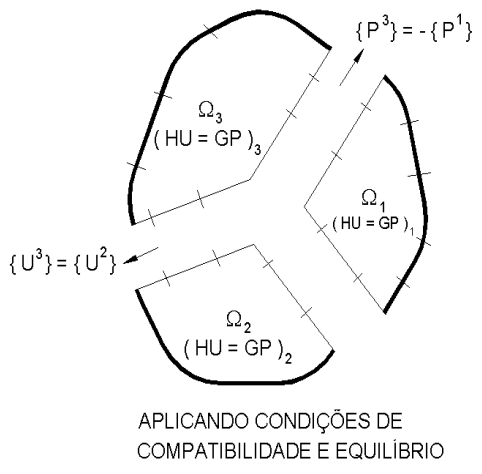

Figura 2.11 - a) Representação de um sólido não -homogêneo. b) Condições de interface entre regiões. 
A análise do sólido não-homogêneo é realizada, similarmente ao método dos elementos finitos, fazendo-se a montagem do sistema a partir das contribuições de cada sub-região. As incógnitas de deslocamentos e de forças de superfície nas interfaces em comum são eliminadas aplicando-se as equações de compatibilidade e equilíbrio do modelo cinemático adotado.

Assim, gera-se um sistema final, já impostas as condições de contorno da forma:

$$
A \cdot X=B
$$

onde a matriz $A$ possui dimensão igual à soma de todas as dimensões dos subdomínios, subtraindo-se uma equação comum em cada grau de liberdade da interface. Esta matriz, em geral, se torna mais esparsa à medida que os diversos subdomínios estejam mais desacoplados um do outro. Os vetores $B$ e $X$ contêm, respectivamente, as incógnitas e os valores conhecidos do contorno do sólido formado por várias sub-regiões.

\subsection{Exemplos numéricos dos casos tridimensionais}

Para avaliar a performance do código computacional desenvolvido na análise de estruturas tridimensionais pelo MEC, são apresentados neste item 4 exemplos buscados na literatura e comparados com os resultados analíticos ou com os conseguidos em trabalhos usando o MEC ou o MEF.

Assim, em cada item é apresentada a geometria do problema, seus parâmetros físicos, a rede de elementos adotada para os exemplos e os resultados encontrados pelo código desenvolvido e os encontrados por outros autores ou empregando o programa comercial Ansys.

Uma questão que deve ser ressaltada é em relação a calibração da densidade da subelementação utilizada que, para cada exemplo, foi feita experimentalmente mediante o refino progressivo da densidade até se chegar ao ponto ótimo. Isto ainda não representa o melhor procedimento a ser adotado e pretende-se mais para frente desenvolver um estudo em que se possa, mediante 
o cálculo do erro dado pela parcela singular associado a distância do ponto fonte, avaliar a melhor densidade a ser adotada.

Em todos os exemplos a seguir foram empregues elementos de superfície triangulares planos e com aproximação linear tanto para os deslocamentos como para as forças de superfície. $O$ valor $L$ que aparece nos exemplos a seguir se refere à média entre os lados do elemento triangular.

\subsubsection{Sólido tracionado}

Neste exemplo se faz a análise de um sólido paralelepipédico sendo tracionado em uma direção com força distribuída com valor unitário. A figura 2.12 esquematiza a geometria e as características elásticas do sólido além da rede admitida para o contorno.

Esse exemplo avalia um estado puro de tração, e para este caso se conhece com exatidão a distribuição dos deslocamentos e das tensões. Como a distribuição de deformações é constante, ou seja, tem-se uma variação linear dos deslocamentos e que o elemento adotado também apresenta esta variação, a resposta avaliada pelo MEC teria que ser exata.

A técnica convencional utilizada para a subelementação é a de dividir de forma regular os elementos próximos ao ponto de colocação (vide figura 2.6), denominada de SC. Uma correlação bastante utilizada é avaliar a distância (R) que vai do ponto fonte ao CG do elemento, e comparar este valor com a média dos comprimentos $\left(L_{m}\right)$ do elemento a ser integrado. Esta correlação é dada por BARBIRATO (1999) como:

\begin{tabular}{|l|l|}
\hline $0 \leq R \leq L_{m}$ & 25 subelementos \\
\hline$L_{m}<R \leq 2 L_{m}$ & 16 subelementos \\
\hline $2 L_{m}<R \leq 4 L_{m}$ & 9 subelementos \\
\hline$R>4 L_{m}$ & 4 subelementos \\
\hline
\end{tabular}

Foi desenvolvido um procedimento em que se pode especificar um número qualquer de subelementos para a realização da integração numérica, assim as 
tabelas 2.1 e 2.2 apresentam respostas para casos onde se emprega o algoritmo dado pela correlação acima. Entretanto, para o cálculo dos valores internos foram empregadas as seguintes correlações:

\begin{tabular}{|l|l|}
\hline $0 \leq R \leq L_{m}$ & 225 subelementos \\
\hline$L_{m}<R \leq 2 L_{m}$ & 196 subelementos \\
\hline $2 L_{m}<R \leq 4 L_{m}$ & 169 subelementos \\
\hline$R>4 L_{m}$ & 144 subelementos \\
\hline
\end{tabular}

Assim, avaliam-se as respostas do contorno e de valores internos no sólido. Comparam-se as respostas exatas com as obtidas pelas diferentes técnicas de integração do elemento singular, onde para os elementos não-singulares, emprego-se ou a correlação dada por BARBIRATO (1999) ou a aplicada pela última correlação apresentada.

Para os elementos quase-singulares, compararam-se as respostas obtidas com o uso das integrações numéricas computadas via subelementação progressiva (SP) e pela subelementação convencional (SC), onde as densidades de subelementos usadas para as duas técnicas são mostradas na figura 2.13 .

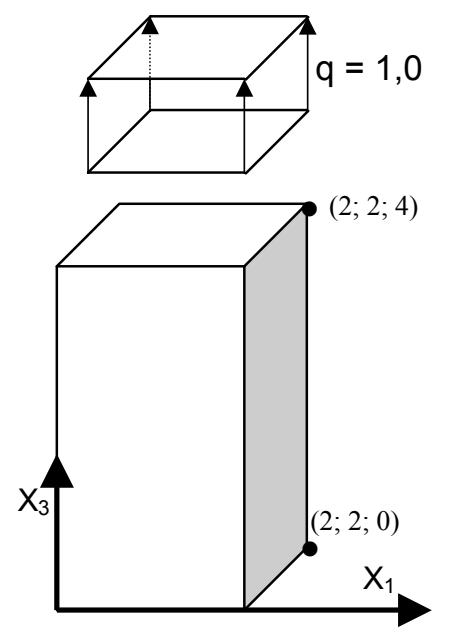

Pontos:

A $(1 ; 1 ; 4)$

$\mathrm{B}(1 ; 1 ; 2)$

$\mathrm{C}(1 ; 1 ; 0,01)$

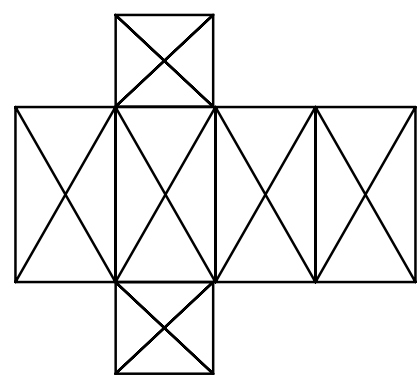

Figura 2.12 - Sólido apoiado em sua base (plano $X_{1} X_{2}$ e $X_{3}=0$ ) e com valor de $E=1$ e $v=0,25$. Rede adotada para o contorno e coordenadas de pontos de interesse. 
Tabela 2.1:

Deslocamentos $\mathrm{u}_{3}$ dos pontos $\mathrm{A}, \mathrm{B}$ e $\mathrm{C}$ do sólido.

\begin{tabular}{|c|c|c|c|c|c|}
\hline Exato & Semi - analítico & $\mathbf{0 , 0 5 L}(\mathbf{S P})$ & $\mathbf{0 , 0 1 L} \mathbf{( S P )}$ & $\mathbf{0 , 0 5 L}(\mathbf{S C})$ & $\mathbf{0 , 0 1 L}(\mathbf{S C})$ \\
\hline $\mathrm{A}(4,0)$ & 4,0000 & 4,0006 & 4,0034 & 4,0590 & 3,8268 \\
$\mathrm{~B}(2,0)$ & 2,0001 & 2,0002 & 1,9953 & 1,9985 & 2,4440 \\
\hline
\end{tabular}

Tabela 2.2:

Tensões $\sigma_{33}$ do sólido.

\begin{tabular}{|c|c|c|c|c|c|}
\hline Exato & $\begin{array}{c}\text { Semi - analítico } \\
\text { com SC }\end{array}$ & $\mathbf{0 , 0 5 L}$ (SP) & $\mathbf{0 , 0 1 L}$ (SP) & $\mathbf{0 , 0 5 L ~ ( S C ) ~}$ & $\mathbf{0 , 0 1 L ~ ( S C ) ~}$ \\
\hline B $(1,0)$ & 1,0000 & 1,0001 & 0,9995 & 0,9994 & 1,3303 \\
C $(1,0)$ & 0,7817 & 1,0345 & 1,02826 & 0,7829 & 0,8824 \\
\hline
\end{tabular}

Figura 2.13 - Subelementações empregadas.

Os resultados apresentados pelas tabelas 2.1 e 2.2 demonstram boa concordância com os valores exatos do problema. A técnica semi-analítica mostrou resultados mais próximos dos exatos. Entretanto, para o caso de se calcular as tensões internas próximas ao contorno, ponto $C$, a integração numérica usada quando se utiliza a correlação apresentada em BARBIRATO (1999), levou a um erro relativo de $-21,83 \%$, mas ao se empregar a correlação apresentada por esse trabalho esse erro caiu para $3,45 \%$. 
Com respeito ao uso das técnicas de integração numérica com subelementação, nota-se também muito boa concordância com os resultados analíticos. Esse exemplo não mostrou diferenças significativas quando se emprega ou a SC ou a SP. Sendo preferível, nesse caso, usar a técnica de subelementação convencional, uma vez que o custo computacional é menor. Ressalta-se que para a distância de 1/100 da média dos lados de cada elemento do problema entre o ponto fonte o elemento quase-singular, o uso da SC não apresentou bons resultados.

Para o caso de se empregar a técnica de integração convencional, deve-se tomar o mesmo procedimento que cálculo de tensões internas usada na técnica semi-analítica, onde nesse caso a tensão $\left(\sigma_{33}\right)$ para o ponto $C$ o erro cai para $3,75 \%$.

\subsubsection{Viga elástica solicitada à flexão}

Apresenta-se neste item uma viga elástica engastada em uma extremidade e na outra sendo aplicada uma força perpendicular. A figura 2.14 apresenta os parâmetros elásticos/mecânicos e a geometria da viga. Pretende-se com esse exemplo avaliar o desempenho da formulação aplicada a um problema que envolve o efeito de flexão na análise de um problema em que uma dimensão é superior as outras duas.

Na figura 2.15 é esquematizada a rede (malha) aplicada para discretizar o contorno, onde a rede possui 72 elementos e 46 nós. São apresentadas, também, as respostas obtidas quando se usa o programa comercial Ansys, onde o tipo de elemento escolhido é o Solid45 que possui a geometria ou de um sólido tetraedro ou de um hexaedro, e ele contêm 8 nós por elemento, onde se empregou um total de 2000 elementos.

A tabela 2.3 apresenta as resultados obtidos pelas presentes técnicas de integração semi-analítica e subelementação progressiva, de maneira que as integrais quase-singulares são obtidas usando-se a correlação apresentada por BARBIRATO (1999). Essas respostas são confrontadas com as obtidas pelas técnicas usadas por SOUZA et al. (2002), e pela técnica de subelementação 
regular usada por BARBIRATO (1999), sendo que esses autores também usam para os elementos não-singulares a correlação citada acima. Destaca-se que BARBIRATO (1999) emprega a mesma rede, mas usando nós descontínuos para todos os elementos, portanto, possuindo um total de 216 nós. A subelementação empregada para a integração progressiva é a mesma que se usou no exemplo anterior (vide figura 2.13), apenas mudando o fator da distância do ponto fora para 0,1 vezes a média dos lados de cada elemento.
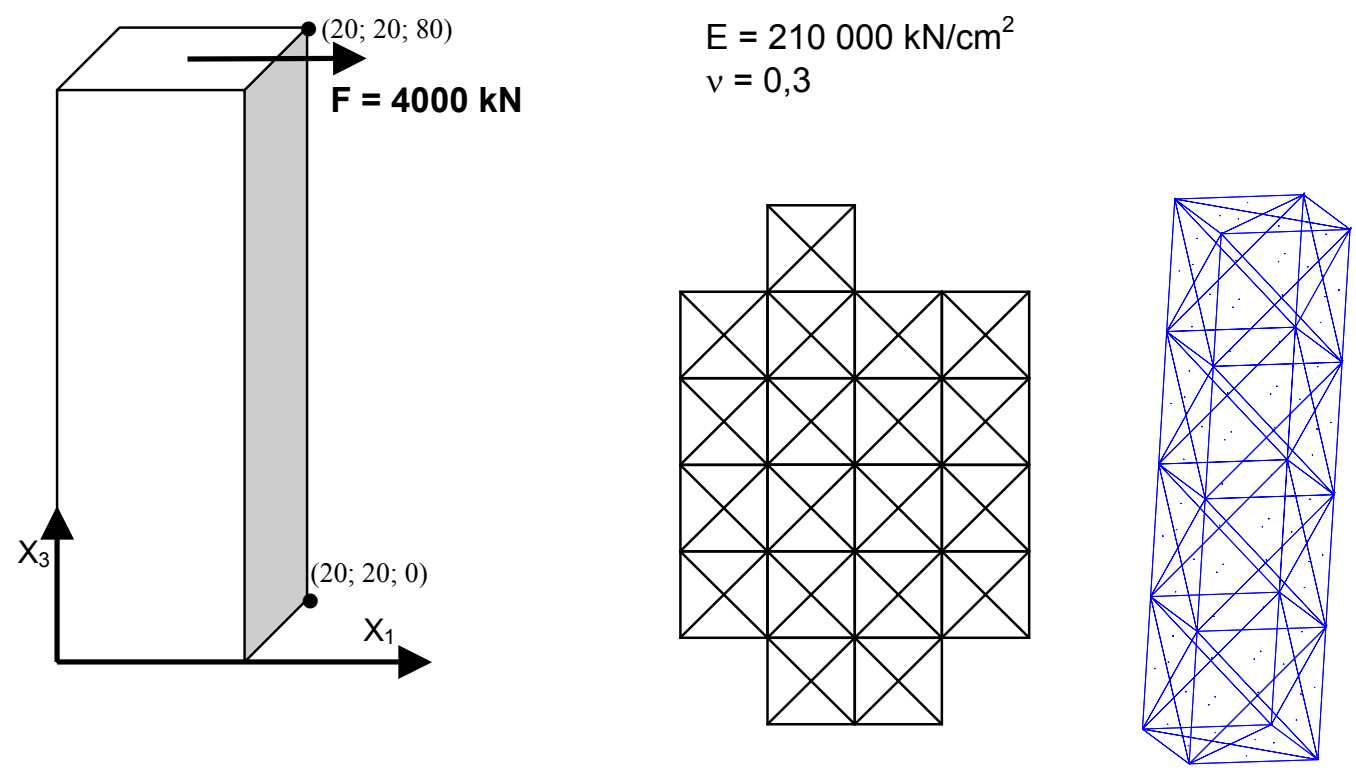

Figura 2.14 - Geometria e valores elásticos da viga engastada no plano $\left(X_{1} X_{2}\right.$ e $\left.X_{3}=0\right)$, e configuração da rede.

Tabela 2.3:

Linha elástica do sólido analisado $(\mathrm{cm})$.

\begin{tabular}{|c|c|c|c|c|c|c|}
\hline $\begin{array}{c}\text { Eixo } \\
\text { médio } \\
\left(10 ; 10 ; X_{3}\right)\end{array}$ & $\begin{array}{c}\text { Numérica } \\
\left(0,1^{*} \mathrm{~L}\right)\end{array}$ & $\begin{array}{c}\text { Semi - } \\
\text { analítico }\end{array}$ & $\begin{array}{c}\text { SOUZA } \\
\text { et al. (2002) } \\
\text { [ISQG] }\end{array}$ & $\begin{array}{c}\text { SOUZA } \\
\text { et al. (2002) } \\
\text { [HMEC] }\end{array}$ & $\begin{array}{c}\text { Barbirato } \\
\text { (1999) }\end{array}$ & $\begin{array}{l}\text { Ansys } \\
\text { Solid45 }\end{array}$ \\
\hline 80 & 0,2520 & 0,2032 & 0,2507 & 0,2439 & 0,2350 & 0,2521 \\
\hline 60 & 0,1616 & 0,1331 & 0,1602 & 0,1551 & 0,1490 & 0,1606 \\
\hline 40 & 0,0819 & 0,0707 & 0,0808 & 0,0773 & 0,0740 & 0,0805 \\
\hline 20 & 0,0241 & 0,0221 & 0,0236 & 0,0217 & 0,0210 & 0,0231 \\
\hline
\end{tabular}


Tabela 2.4:

Tensão vertical $\left(\sigma_{33}\right)$ para 3 situações.

\begin{tabular}{|c|c|c|c|}
\hline Ponto & $\begin{array}{c}\text { Presente correlação } \\
\text { para pontos internos }\end{array}$ & $\begin{array}{c}\text { Correlação de Barbirato (1999) para } \\
\text { pontos internos }\end{array}$ & $\begin{array}{c}\text { Ansys } \\
\text { Solid45 }\end{array}$ \\
\hline $\mathrm{A}(0,5 ; 10 ; 52)$ & 84,48 & 2876,90 & 76,37 \\
$\mathrm{~B}(5 ; 10 ; 52)$ & 40,83 & 40,83 & 44,92 \\
\hline
\end{tabular}

As respostas obtidas na análise pelo Ansys devem ser muito próximas dos valores exatos, pois à medida que se aumentava o refinamento $h$ da rede do sólido, os valores convergiam aos apresentados pelas tabelas 2.3 e 2.4. Assim, na tabela 2.3 é possível notar que os deslocamentos obtidos quando se emprega a técnica da subelementação progressiva se aproximam bastante dos valores de comparação. Onde para os deslocamentos máximos do sólido, as diferenças relativas foram de $0,04 \%$. Para o caso das tensões $\left(\sigma_{33}\right)$, os valores apresentaram diferenças maiores que as obtidas para os casos dos deslocamentos, sendo estas diferenças para o ponto $A$ de $+10,62 \%$ e de $-9,10 \%$ para o ponto $B$, quando se emprega a correlação proposta nesse trabalho.

É possível se verificar na tabela 2.4 que a correlação empregada por BARBIRATO (1999) demonstra que para os pontos internos muito próximos do contorno ela é inadequada, enquanto que a correlação aqui utilizada mostra-se uma maior estabilidade nas repostas para estes pontos mais próximos do contorno.

\subsubsection{Ensaio de Kérisel \& Adam (1967)}

Este exemplo utiliza a técnica da sub-região convencional para simular a interação entre o solo e a estaca. Para a integração quase-singular é empregada a técnica da subelementação progressiva, de modo que neste e no próximo exemplo o fator da distância do ponto fora é 1/100 vezes a média dos lados de cada elemento, e a densidade da distribuição de subelementos utilizada é a apresentada na figura 2.13 .

O exemplo é baseado no ensaio de KÉRISEL \& ADAM (1967), os quais analisam uma estaca cravada em solo argiloso, onde o comprimento da estaca é 
de 4,65m e seu diâmetro de 0,3573m, e os demais dados do problema são apresentados na figura 2.15 .

O solo é representado como um meio semi-infinito, de modo que se empregou uma rede de elementos apenas na superfície livre do meio e ao longo da superfície da estaca. Como a formulação de contorno, via Kelvin, não prescreve, a priori, as condições nulas de forças na região não-carregada, é imperativo discretizar esta superfície livre até distâncias consideradas não perturbadas. A figura 2.16 apresenta esta rede adotada do solo e da estaca, e esta última é representada como um elemento na forma de um prisma quadrangular, onde cada face lateral é discretizada por dois elementos triangulares.

Obtiveram-se, assim, os resultados de deslocamentos horizontais e forças de contato verticais entre solo/estaca, representados, respectivamente, nas figuras 2.17 e 2.18. Na figura 2.17 nota-se uma boa concordância entre os valores de deslocamentos na presente formulação e os obtidos pelo ensaio à medida que se melhora a discretização da estaca.

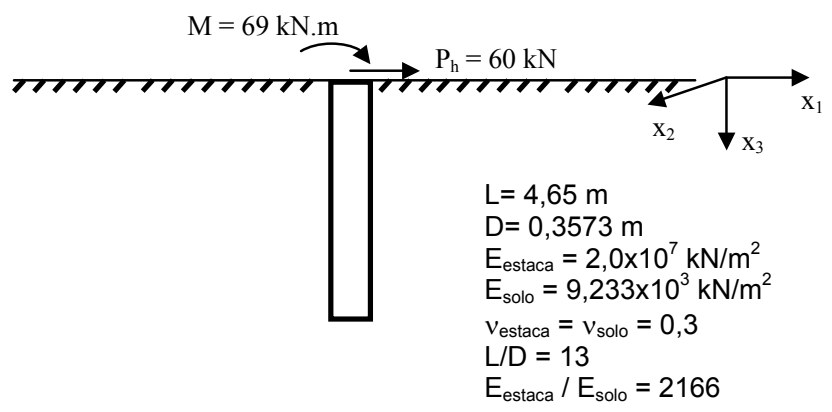

Figura 2.15 - Estaca submetida à força horizontal e momento fletor, parâmetros físicos e geométricos. 

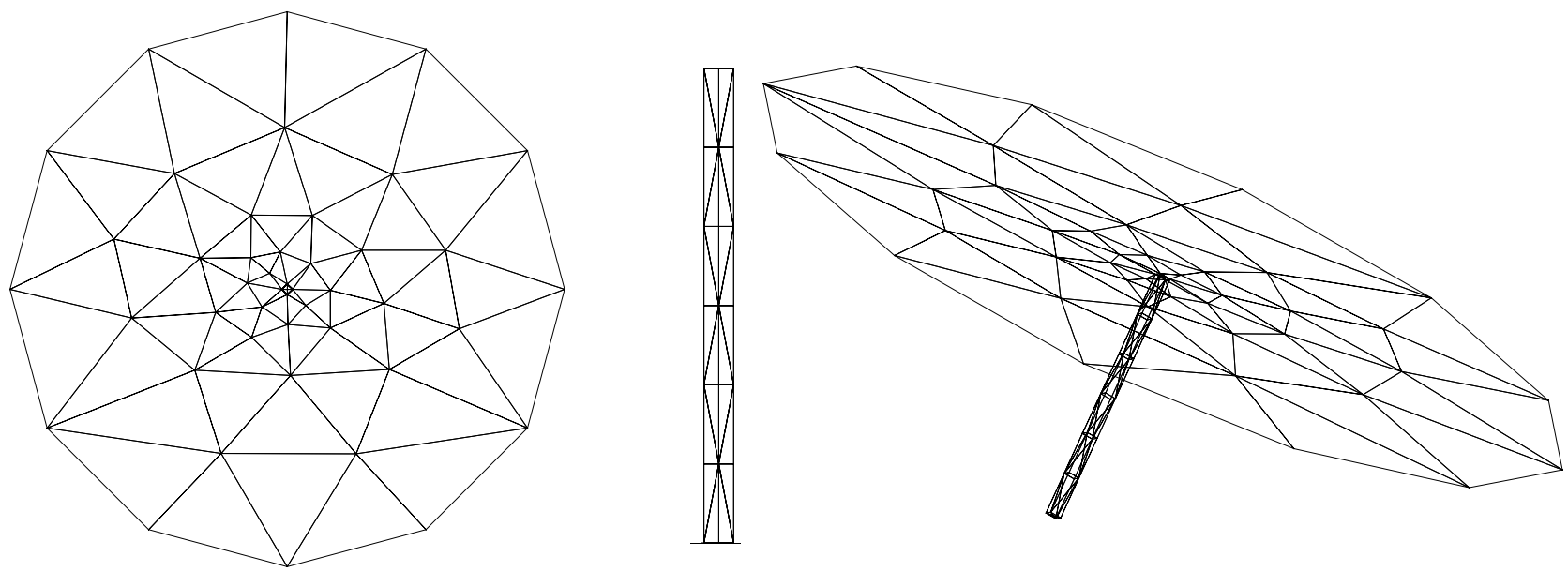

Figura 2.16 - Configurações da rede adotada para o solo e a estaca.

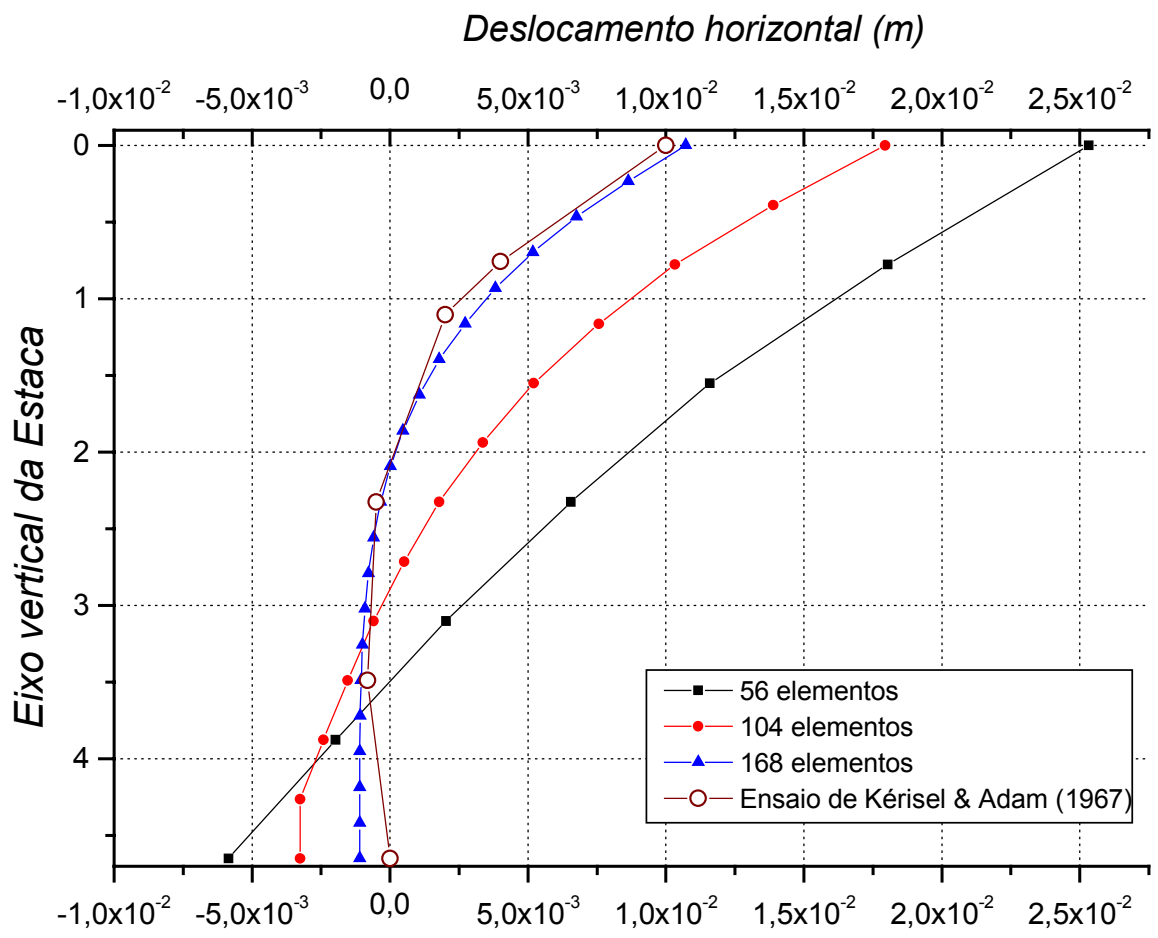

Figura 2.17 - Deslocamentos horizontais ao longo do eixo da estaca. 


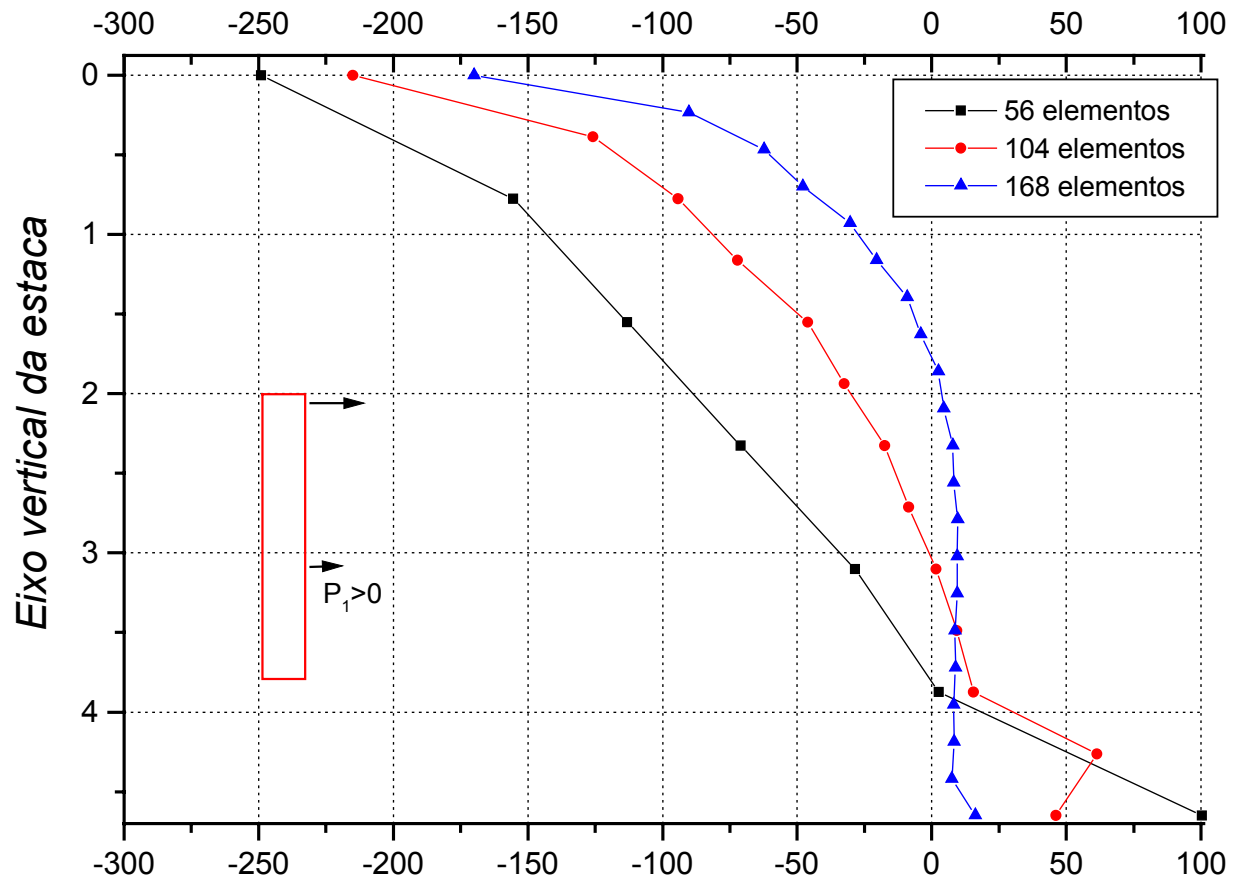

Figura 2.18 - Forças de contato horizontais $\left(\mathrm{kN} / \mathrm{m}^{2}\right)$ ao longo do eixo da estaca.

\subsubsection{Ensaio de Whitaker \& Cooke}

Este exemplo é extraído de FERRO (1993), o qual compara sua formulação com o ensaio de WHITAKER \& COOKE, apresentado em POULOS \& DAVIS (1980). Os pesquisadores ensaiaram uma estaca, os seus dados são mostrados na figura 2.19, bem como uma perspectiva do elemento de fundação adotado pelo presente trabalho. FERRO (1993) avalia este exemplo empregando uma combinação do $\mathrm{MEC} / \mathrm{MEF}$, de modo que o solo é representado via solução de Mindlin e a estaca com elemento tridimensional de barra. 

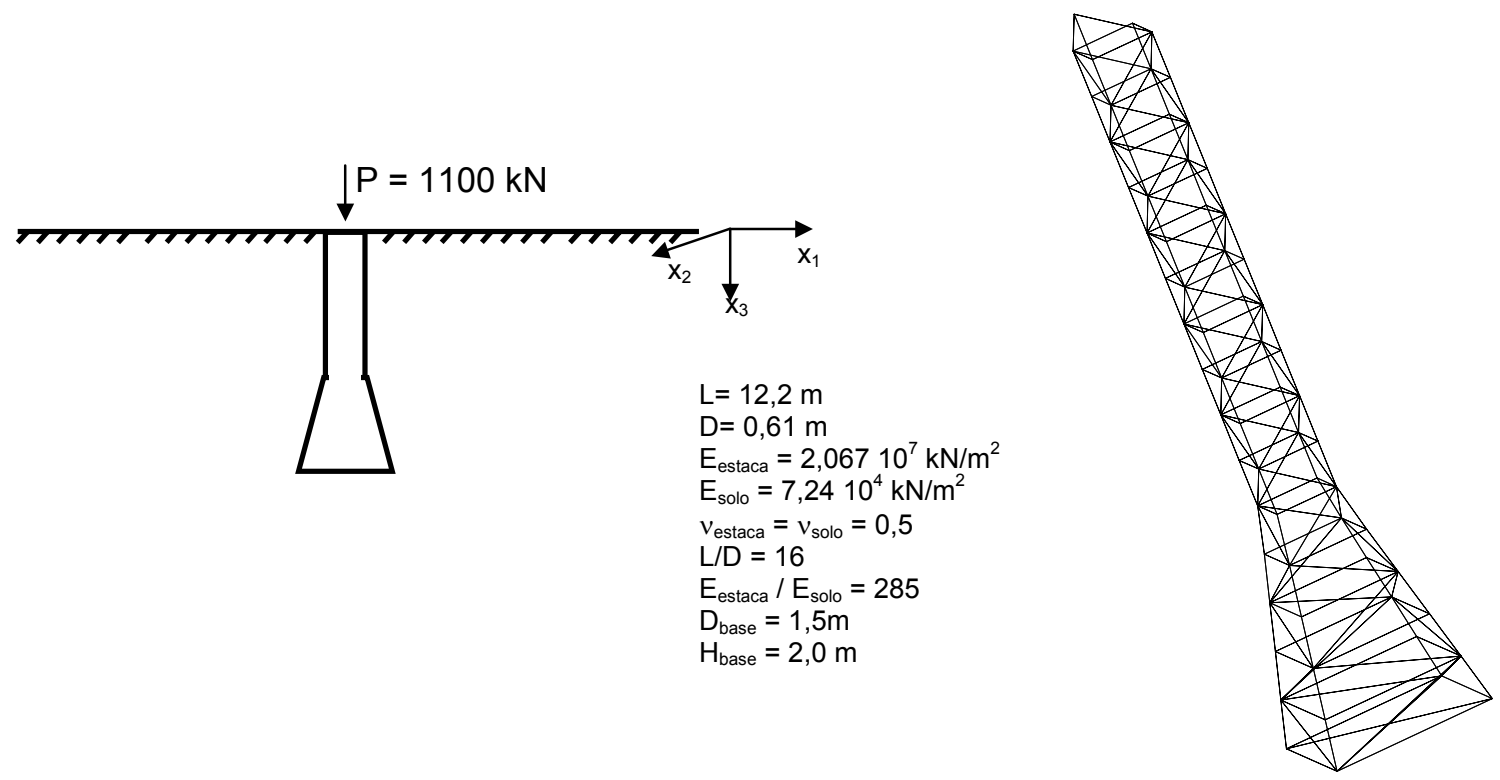

Figura 2.19 - Elemento de fundação submetido à força vertical, parâmetros físicos e geométricos e vista da rede empregada para simular a estaca ou o tubulão.

São comparadas com a presente formulação as respostas obtidas por FERRO (1993) em termos de deslocamentos da estaca apresentada na figura (2.19). Além disso, também são apresentadas as respostas obtidas com o alargamento da base da estaca, onde na figura (2.19) é verificado a geometria deste tubulão. O deslocamento de topo obtido no ensaio foi de $0,284 \mathrm{~cm}$, e o obtido com o uso de 248 elementos foi de 0,289 , ou seja, uma diferença relativa de $1,76 \%$, e FERRO (1993) obtém o valor de $0,29 \mathrm{~cm}$. Quando se considera o elemento de fundação com alargamento de base (tubulão), o deslocamento de topo encontrado foi $0,277 \mathrm{~cm}$. As figuras 2.21 e 2.22 mostram a distribuição das forças de contato cisalhante ao longo do fuste, e a distribuição das forças de contato normal à superfície da ponta da estaca e do tubulão. 


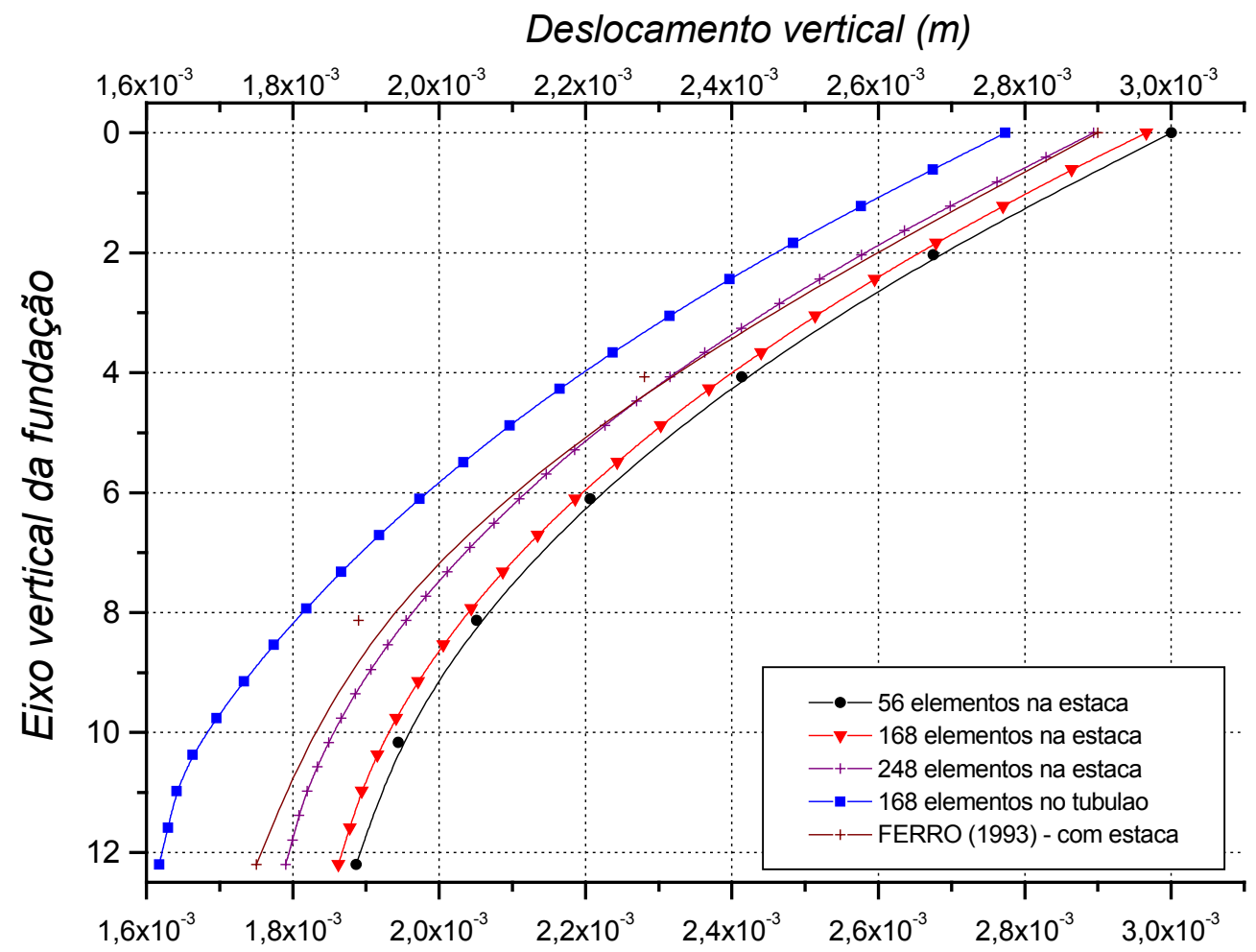

Figura 2.20 - Deslocamentos verticais ao longo do eixo da estaca e do tubulão.

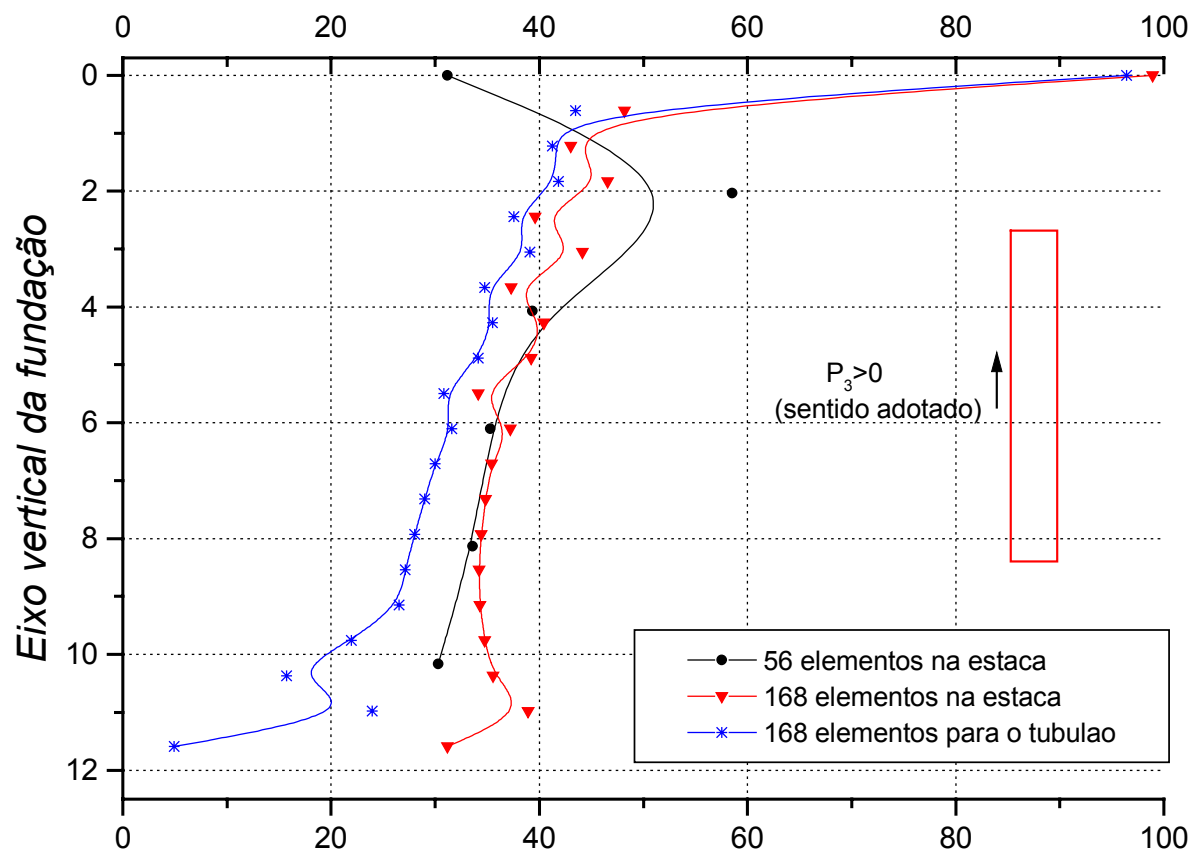

Figura 2.21 - Forças de contato cisalhante $\left(\mathrm{kN} / \mathrm{m}^{2}\right)$ ao longo do eixo da estaca. 

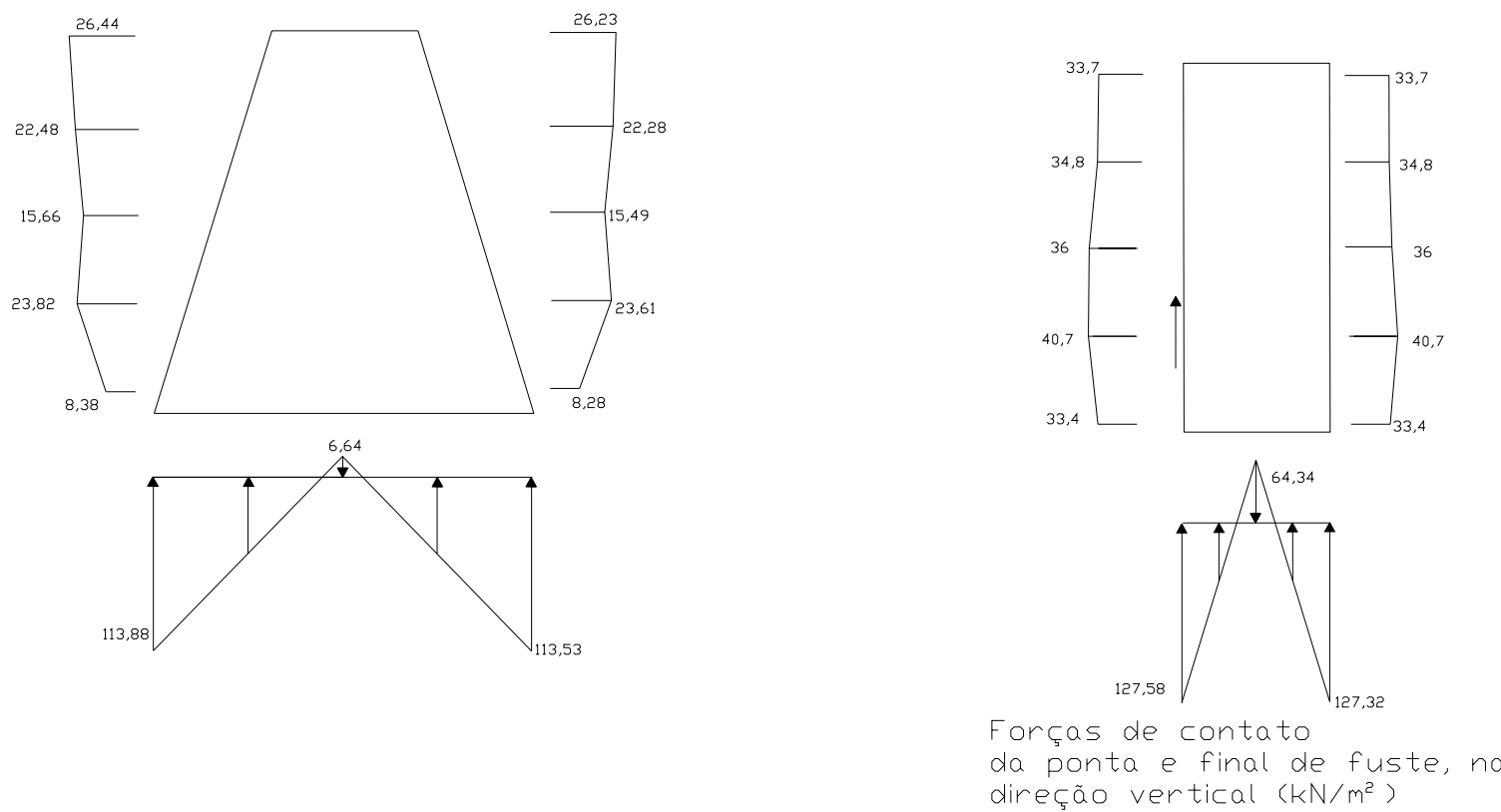

Figura 2.22 - Forças de contato cisalhante e normal na região do fuste e da ponta do tubulão e da estaca. 


\section{Capítulo 3}

\section{FORMULAÇÃO DO SOLO NÃO-HOMOGÊNEO ENRIJECIDO POR ELEMENTO DE FUNDAÇÃO}

\subsection{Introdução}

Este capítulo apresenta no item 3.2 a formulação do solo nãohomogêneo sem a consideração dos elementos de fundação. Ela é baseada na técnica empregada por MAIER \& NOVATI (1987), denominada de técnica da "rigidez sucessiva". Este nome advém da semelhança obtida pelo sistema final de equações com o que aparece no MEF, ou seja, uma relação que associa as ações externas diretamente com os deslocamentos. Entretanto, a matriz resultante do sistema linear, ao contrário do $M E F$, não atende as exigências requeridas para a correta definição do termo "matriz de rigidez".

A denominação "sucessiva" configura a representação de um sistema de equações que é gerado sucessivamente entre dois domínios adjacentes, a partir das condições de equilíbrio e compatibilidade - até se atingir a região que está em contato com a superfície externa do solo, já incorporadas nesta última expressão todas as influências dos demais estratos.

No item 3.3, é formulada a técnica da "rigidez sucessiva" introduzindose os domínios que representam os elementos de fundações dentro de cada estrato genérico. O desenvolvimento é particularizado para caso de se

considerar apenas elementos de estacas, mas não se perdendo a generalidade da formulação para os demais casos possíveis, uma vez que o elemento de fundação é representado por elementos tridimensionais.

O item 3.4 visa comparar os números de operações envolvidas quando se aplica o procedimento convencional de sub-região com a técnica 
da "rigidez sucessiva" para a montagem e a resolução do sistema final do problema solo não-homogêneo/fundação.

\subsection{Maciço estratificado pelo método da "rigidez sucessiva"}

Suponha-se um maciço com $\eta$ camadas adjacentes (figura 3.1), cada um com características físicas próprias, mantendo-se dentro de seu domínio as mesmas propriedades elásticas e isótropas.

Para uma camada genérica "i", é possível escrever a relação das matrizes de influência advindas do MEC (equação 2.30), sem considerar forças volumétricas, como:

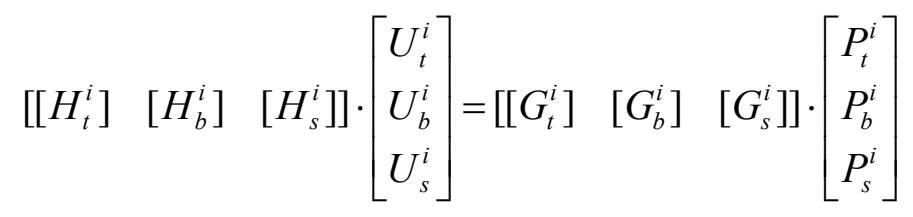
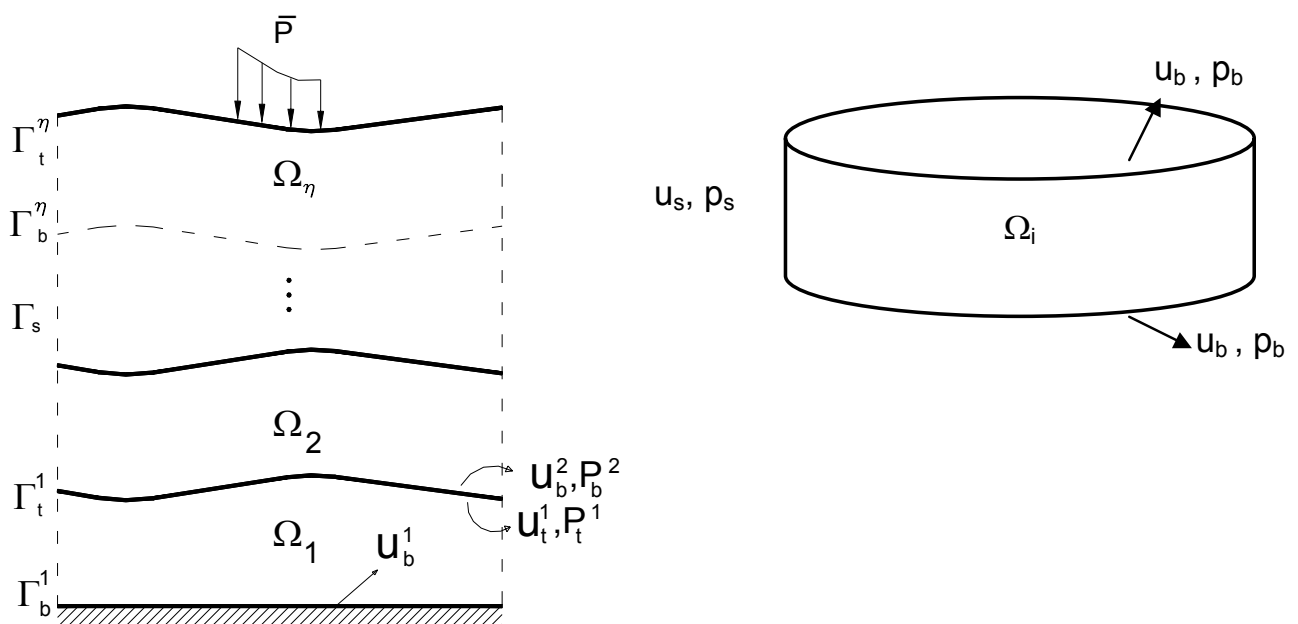

Figura 3.1 - Maciço estratificado sob forças de superfície e contornos de base, topo e lateral.

onde $u$ e $p$ são, respectivamente, os campos de deslocamentos e forças de superfícies, e H e G são as matrizes da equação (2.30). E $t$, $b$ e $s$ são abreviações, respectivamente, de topo, base e superfície lateral.

Expandindo-se a expressão (3.1) para: 


$$
\left[\begin{array}{ccc}
{\left[H_{t t}^{i}\right]} & {\left[H_{t b}^{i}\right]} & {\left[H_{t s}^{i}\right]} \\
{\left[H_{b t}^{i}\right]} & {\left[H_{b b}^{i}\right]} & {\left[H_{b s}^{i}\right]} \\
{\left[H_{s t}^{i}\right]} & {\left[H_{s b}^{i}\right]} & {\left[H_{s s}^{i}\right]}
\end{array}\right] \cdot\left\{\begin{array}{c}
U_{t}^{i} \\
U_{b}^{i} \\
U_{s}^{i}
\end{array}\right\}=\left[\begin{array}{ccc}
{\left[G_{t t}^{i}\right]} & {\left[G_{t b}^{i}\right]} & {\left[G_{t s}^{i}\right]} \\
{\left[G_{b t}^{i}\right]} & {\left[G_{b b}^{i}\right]} & {\left[G_{b s}^{i}\right]} \\
{\left[G_{s t}^{i}\right]} & {\left[G_{s b}^{i}\right]} & {\left[G_{s s}^{i}\right]}
\end{array}\right] \cdot\left\{\begin{array}{c}
P_{t}^{i} \\
P_{b}^{i} \\
P_{s}^{i}
\end{array}\right\}
$$

Podem-se impor as condições de compatibilidade de deslocamentos e equilíbrio das forças de contato que surgem ao longo do contorno entre as camadas adjacentes. Assim, para os deslocamentos tem-se a relação de igualdade cinemática entre a camadas " $"$ " e " $i+1$ ":

$$
u_{t}^{i}=u_{b}^{i+1}
$$

Para as condições de equilíbrio, tem-se:

$$
p_{t}^{i}=-p_{b}^{i+1}
$$

Admitindo-se, para o contorno lateral, que este não seja perturbado, ou seja:

$$
u_{s}^{i}=0
$$

e a terceira equação de (3.2) é escrita como:

$$
\left[H_{s t}^{i}\right] \cdot\left\{U_{t}^{i}\right\}+\left[H_{s b}^{i}\right] \cdot\left\{U_{b}^{i}\right\}=\left[G_{s s}^{i}\right] \cdot\left\{P_{s}^{i}\right\}
$$

Ou isolando-se as forças de superfície da lateral não perturbada, chega-se a:

$$
\left\{P_{s}^{i}\right\}=\left[G_{s s}^{i}\right]^{-1} \cdot\left[H_{s t}^{i}\right] \cdot\left\{U_{t}^{i}\right\}+\left[G_{s s}^{i}\right]^{-1} \cdot\left[H_{s b}^{i}\right] \cdot\left\{U_{b}^{i}\right\}
$$

Desta maneira, após serem calculados os deslocamentos de base e de topo de cada camada, podem-se obter as forças de superfície ao longo de sua lateral.

As equações da relação (3.2) podem ser escritas isolando-se as forças de superfície, ou seja: 


$$
\left\{\begin{array}{c}
P_{t}^{i} \\
P_{b}^{i}
\end{array}\right\}=\left[\begin{array}{cc}
{\left[K_{t t}^{i}\right]} & {\left[K_{t b}^{i}\right]} \\
{\left[K_{b t}^{i}\right]} & {\left[K_{b b}^{i}\right]}
\end{array}\right] \cdot\left\{\begin{array}{c}
U_{t}^{i} \\
U_{b}^{i}
\end{array}\right\}
$$

onde a matriz da expressão (3.8) é dada por:

$$
\left[\begin{array}{ll}
{\left[K_{t t}^{i}\right]} & {\left[K_{t b}^{i}\right]} \\
{\left[K_{b t}^{i}\right]} & {\left[K_{b b}^{i}\right]}
\end{array}\right]=\left[\begin{array}{ll}
{\left[G_{t t}^{i}\right]} & {\left[G_{t b}^{i}\right]} \\
{\left[G_{b t}^{i}\right]} & {\left[G_{b b}^{i}\right]}
\end{array}\right]^{-1} \cdot\left[\begin{array}{ll}
{\left[H_{t t}^{i}\right]} & {\left[H_{t b}^{i}\right]} \\
{\left[H_{b t}^{i}\right]} & {\left[H_{b b}^{i}\right]}
\end{array}\right]
$$

Agora, já com as relações desenvolvidas para duas camadas subseqüentes: "i” e “i+1". Após considerar o equilíbrio, compatibilidade e deslocamentos nulos da lateral das camadas, pode-se partir da última camada, que será considerada com seus deslocamentos nulos, ou seja, base indeslocável, e utilizar as expressões de (3.1) a (3.9) para ir condensando as influências de cada camada em sua adjacente superior, até se chegar à equação (3.8) da última camada que contempla forças de superfície prescritas, para o caso de carregamento diretamente sobre a superfície, ou à qual poderá se acoplar com as forças de reações do radier ou da superestrutura.

Ou seja, esquematicamente para o exemplo representado na figura (3.1), tem-se:

\section{$1^{a}$ camada:}

Considerando-se esta camada com deslocamento de base nulo, ou seja, meio indeslocável, reescreve-se a relação (3.8):

$$
\begin{aligned}
& \left\{P_{t}^{1}\right\}=\left[K_{t t}^{1}\right] \cdot\left\{U_{t}^{1}\right\} \\
& \left\{P_{b}^{1}\right\}=\left[K_{b t}^{1}\right] \cdot\left\{U_{t}^{1}\right\}
\end{aligned}
$$

\section{$2^{a}$ camada:}

A relação (3.8) pode ser escrita da seguinte maneira:

$$
\begin{aligned}
& \left\{P_{t}^{2}\right\}=\left[K_{t t}^{2}\right] \cdot\left\{U_{t}^{2}\right\}+\left[K_{t b}^{2}\right] \cdot\left\{U_{b}^{2}\right\} \\
& \left\{P_{b}^{2}\right\}=\left[K_{b t}^{2}\right] \cdot\left\{U_{t}^{2}\right\}+\left[K_{b b}^{2}\right] \cdot\left\{U_{b}^{2}\right\}
\end{aligned}
$$


E como as camadas 1 e 2 são adjacentes:

$$
\left\{P_{t}^{1}\right\}=-\left\{P_{b}^{2}\right\}
$$

e

$$
\left\{U_{t}^{1}\right\}=\left\{U_{b}^{2}\right\}
$$

Assim, considerando-se as relações (3.1) e (3.15) em (3.13), e isolando-se os deslocamentos da base, tem-se:

$$
\left\{U_{b}^{2}\right\}=-\left[\left(K_{t t}^{1}+K_{b b}^{2}\right)^{-1} \cdot K_{b t}^{2}\right] \cdot\left\{U_{t}^{2}\right\}
$$

Substituindo-se esta expressão em (3.12), chega-se a:

$$
\left\{P_{t}^{2}\right\}=\left[\left[K_{t t}^{2}\right]-\left[K_{t b}^{2}\right] \cdot\left(\left[K_{t t}^{1}\right]+\left[K_{b b}^{2}\right]\right)^{-1} \cdot\left[K_{b t}^{2}\right]\right] \cdot\left\{U_{t}^{2}\right\}
$$

Ou, de forma compacta:

$$
\left\{P_{t}^{2}\right\}=\left[\hat{K}^{2}\right] \cdot\left\{U_{t}^{2}\right\}
$$

Esse procedimento é realizado sucessivamente até se chegar na última camada $(\eta)$, então se tem:

\section{$\eta^{a}$ camada:}

Equilibrando-se e compatibilizando-se as camadas $\eta$ e $\eta-1$, chega-se a:

$$
\begin{aligned}
& \left\{P_{t}^{\eta}\right\}=\left[K_{t t}^{\eta}\right] \cdot\left\{U_{t}^{\eta}\right\}+\left[K_{t b}^{\eta}\right] \cdot\left\{U_{b}^{\eta}\right\} \\
& \left\{P_{b}^{\eta}\right\}=\left[K_{b t}^{\eta}\right] \cdot\left\{U_{t}^{\eta}\right\}+\left[K_{b b}^{\eta}\right] \cdot\left\{U_{b}^{\eta}\right\}
\end{aligned}
$$

E, sabe-se que

$$
\left\{P_{t}^{\eta-1}\right\}=-\left\{P_{b}^{\eta}\right\}
$$

E também que 


$$
\left\{U_{t}^{\eta}\right\}=\left\{U_{b}^{\eta}\right\}
$$

Essas relações (3.21) e (3.22), substituídas em (3.20), considerando-se (3.18), tem-se:

$$
\left\{U_{b}^{\eta}\right\}=-\left[\left(\hat{K}^{\eta-1}+K_{b b}^{\eta}\right)^{-1} \cdot K_{b t}^{\eta}\right] \cdot\left\{U_{t}^{\eta}\right\}
$$

Substituindo-se esta última expressão em (3.19), chega-se a:

$$
\left\{P_{t}^{\eta}\right\}=\left[\left[K_{t t}^{\eta}\right]-\left[K_{t b}^{\eta}\right] \cdot\left(\left[\hat{K}^{\eta-1}\right]+\left[K_{b b}^{\eta}\right]\right)^{-1} \cdot\left[K_{b t}^{\eta}\right]\right] \cdot\left\{U_{t}^{\eta}\right\}
$$

Ou, simplificadamente:

$$
\left\{P_{t}^{\eta}\right\}=\left[\hat{K}^{\eta}\right] \cdot\left\{U_{t}^{\eta}\right\}
$$

Conforme é apresentado na figura 3.1, a última camada está em contato com a superfície livre do solo, onde se deve prescrever forças, para o caso da solução fundamental de Kelvin, toda a sua superfície havendo ou não carregamento aplicado. A expressão (3.25) recai na resolução de um sistema linear onde as incógnitas são os deslocamentos da superfície, ou seja:

$$
\left[\hat{K}^{\eta}\right] \cdot\left\{U_{t}^{\eta}\right\}=\left\{\bar{P}_{t}\right\}
$$

onde $\bar{P}_{t}$ pode indicar as forças prescritas diretamente no solo, ou as reações que o solo exerce na estrutura em contato, por exemplo, as forças de reação entre solo/radier. Assim, ou resolve-se o sistema linear, obtendo-se os deslocamentos de contato, ou acopla-se este sistema (3.26) com outros elementos de contato: radier, blocos, caixas d'água, barragens, taludes, edifícios.

Com os deslocamentos da superfície do solo obtidos, retorna-se para as camadas inferiores e calculam-se seus deslocamentos de base e topo, bem como as forças de contato entre camadas e na lateral.

Ou seja: 
$\eta^{a}$ camada:

Conhecendo-se $\left\{U_{t}^{\eta}\right\}$, obtêm-se $\left\{U_{b}^{\eta}\right\}$ com (3.23), $\left\{P_{b}^{\eta}\right\}$ com (3.20) e $\left\{P_{s}^{\eta}\right\} \operatorname{com}(3.7)$.

$2^{a}$ camada:

Sabendo-se que $\left\{U_{t}^{2}\right\}=\left\{U_{b}^{3}\right\}$, obtêm-se $\left\{U_{b}^{2}\right\}$ com (3.16), $\left\{P_{b}^{2}\right\}$ com (3.13) e $\left\{P_{s}^{2}\right\}$ com (3.7).

$1^{a}$ camada:

Sabendo-se que $\left\{U_{t}^{1}\right\}=\left\{U_{b}^{2}\right\}$, obtêm-se $\left\{P_{b}^{1}\right\}$ com (3.11) e $\left\{P_{s}^{1}\right\}$ com (3.7).

Com os deslocamentos e as forças de superfícies conhecidos no contorno de todas as sub-regiões, é possível calcular quaisquer valores de tensão e de deslocamento dentro de cada subdomínio.

\subsection{Maciço estratificado com estacas}

Neste tópico é apresentada a técnica da "rigidez sucessiva", mas já incorporando as estacas dentro do maciço.

O modelo usado leva em conta a montagem da "matriz de rigidez" de cada estrato, sendo neste caso separadas as variáveis do solo e das estacas de cada camada, tanto do topo, quanto da base e quanto do fuste. Desta forma, as condições de compatibilidade e equilíbrio são tomadas de maneira a garantir a continuidade dos elementos de estaca e do solo. Para o fuste, os deslocamentos e as forças de contato da estaca são escritos em função das variáveis do solo. Para este procedimento utilizado, as variáveis do fuste são condensadas na superfície de cada topo e de cada base da respectiva camada, para garantir que as únicas variáveis existentes em cada estrato ainda sejam as relacionadas ao topo e base.

Para maior entendimento da técnica utilizada, é desenvolvido o procedimento da "rigidez sucessiva" para o estrato com a introdução de duas 
estacas (vide figura 3.2). Mostra-se o sistema final gerado para este conjunto e, em seguida, uma generalização do sistema para $\eta$ estacas em cada substrato.

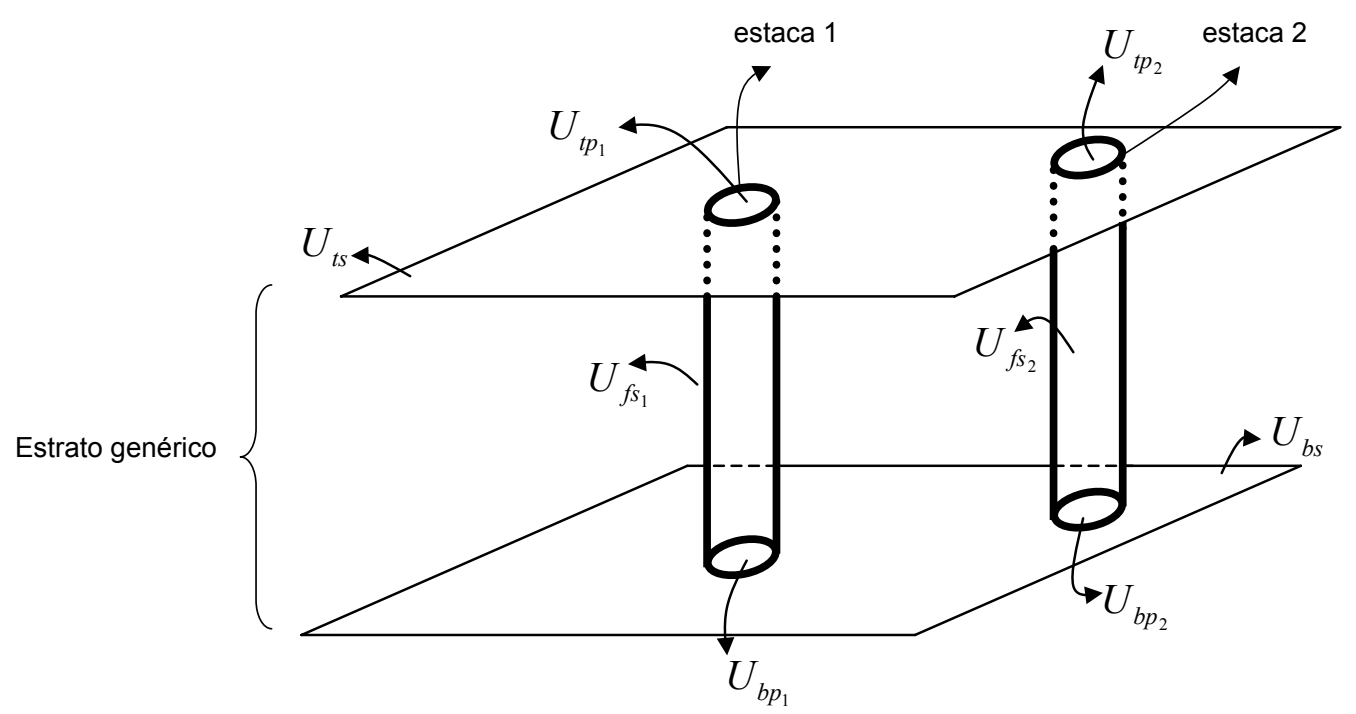

Figura 3.2 - Estrato genérico com duas estacas e seus parâmetros de topo, base e fuste.

Assim, conforme figura 3.2, pode-se escrever para a estaca 1 a relação (2.30) como:

$$
\left[\begin{array}{lll}
{\left[H_{f p_{1}}\right]} & {\left[H_{t p_{1}}\right]} & {\left[H_{b p_{1}}\right]}
\end{array}\right] \cdot\left\{\begin{array}{l}
U_{f p_{1}} \\
U_{t p_{1}} \\
U_{b p_{1}}
\end{array}\right\}=\left[\begin{array}{lll}
{\left[G_{f p_{1}}\right]} & {\left[G_{t p_{1}}\right]} & {\left[G_{b p_{1}}\right]}
\end{array}\right] \cdot\left\{\begin{array}{l}
P_{f p_{1}} \\
P_{t p_{1}} \\
P_{b p_{1}}
\end{array}\right\}
$$

onde o subscrito " $f p_{i}$ " indica fuste do elemento " $i$ ".

Para a estaca 2:

$$
\left[\begin{array}{lll}
{\left[H_{f p_{2}}\right]} & {\left[H_{t p_{2}}\right]} & {\left[H_{b p_{2}}\right.}
\end{array}\right] \cdot\left\{\begin{array}{l}
U_{f p_{2}} \\
U_{t p_{2}} \\
U_{b p_{2}}
\end{array}\right\}=\left[\begin{array}{lll}
{\left[G_{f p_{2}}\right]} & {\left[G_{t p_{2}}\right]} & {\left[G_{b p_{2}}\right.}
\end{array}\right] \cdot\left\{\begin{array}{l}
P_{f p_{2}} \\
P_{t p_{2}} \\
P_{b p_{2}}
\end{array}\right\}
$$

Para uma camada genérica do solo: 


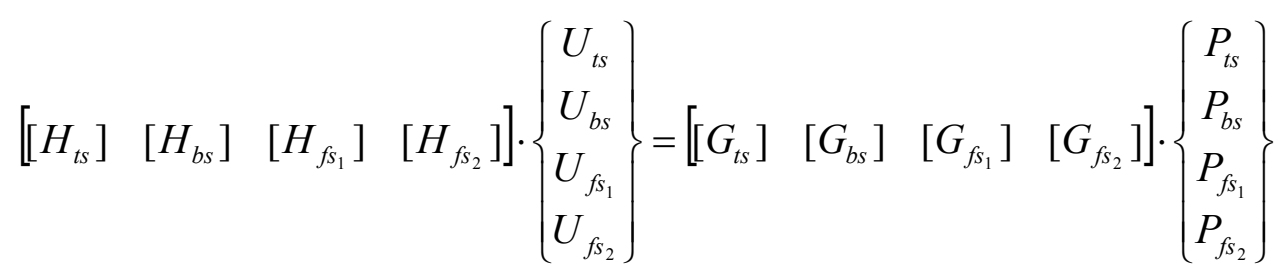

Expandindo (3.29):

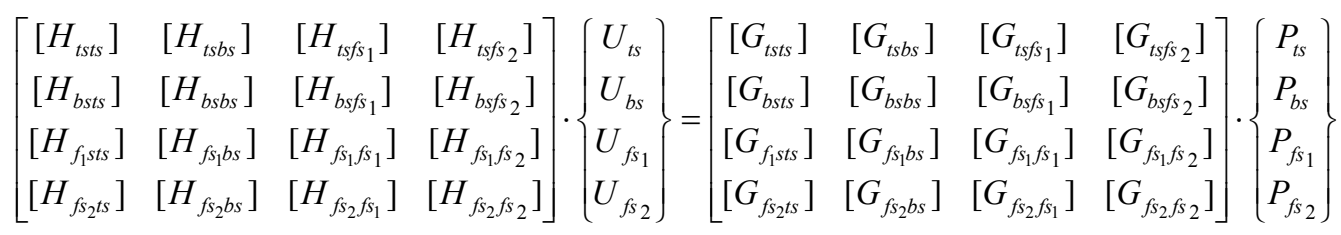

(3.30.1 a 3.30.4)

Expandindo (3.27):

$$
\left[\begin{array}{ccc}
{\left[H_{f p_{1} f p_{1}}\right]} & {\left[H_{f p_{1} t p_{1}}\right]} & {\left[H_{f p_{1} b p_{1}}\right]} \\
{\left[H_{t p_{1} f p_{1}}\right]} & {\left[H_{t p_{1} p_{1}}\right]} & {\left[H_{t p_{1} b p_{1}}\right]} \\
{\left[H_{b p_{1} f p_{1}}\right]} & {\left[H_{b p_{1} p_{1}}\right]} & {\left[H_{b p_{1} b p_{1}}\right]}
\end{array}\right] \cdot\left\{\begin{array}{l}
U_{f p_{1}} \\
U_{t p_{1}} \\
U_{b p_{1}}
\end{array}\right\}=\left[\begin{array}{ccc}
{\left[G_{f p_{1} f p_{1}}\right]} & {\left[G_{f p_{1} t p_{1}}\right]} & {\left[G_{f p_{1} b p_{1}}\right]} \\
{\left[G_{t p_{1} f p_{1}}\right]} & {\left[G_{t p_{1} t p_{1}}\right]} & {\left[G_{t p_{1} b p_{1}}\right]} \\
{\left[G_{b p_{1} f p_{1}}\right]} & {\left[G_{b p_{1} t p_{1}}\right]} & {\left[G_{b p_{1} b p_{1}}\right]}
\end{array}\right] \cdot\left\{\begin{array}{l}
P_{f p_{1}} \\
P_{t p_{1}} \\
P_{b p_{1}}
\end{array}\right\}
$$

Isolando as forças de superfície de (3.31.1):

$P_{f p_{1}}=\left[G_{f p_{1} f p_{1}}\right]^{-1} \cdot\left\{\left[H_{f p_{1} f p_{1}}\right] \cdot U_{f p_{1}}+\left[H_{f p_{1} t p_{1}}\right] \cdot U_{t p_{1}}+\left[H_{f p_{1} b p_{1}}\right] \cdot U_{b p_{1}}-\left[G_{f p_{1} t p_{1}}\right] \cdot P_{t p_{1}}-\left[G_{f p_{1} b p_{1}}\right] \cdot P_{b p_{1}}\right\}$

Expandindo (3.28):

$$
\left[\begin{array}{lll}
{\left[H_{f p_{2} f p_{2}}\right]} & {\left[H_{f p_{2} t p_{2}}\right]} & {\left[H_{f p_{2} b p_{2}}\right]} \\
{\left[H_{t p_{2} f p_{2}}\right]} & {\left[H_{t p_{2} t p_{2}}\right]} & {\left[H_{t p_{2} b p_{2}}\right]} \\
{\left[H_{b p_{2} f p_{2}}\right]} & {\left[H_{b p_{2} t p_{2}}\right]} & {\left[H_{b p_{2} b p_{2}}\right]}
\end{array}\right] \cdot\left\{\begin{array}{l}
U_{f p_{2}} \\
U_{t p_{2}} \\
U_{b p_{2}}
\end{array}\right\}=\left[\begin{array}{ccc}
{\left[G_{f p_{2} f p_{1}}\right]} & {\left[G_{f p_{2} t p_{2}}\right]} & {\left[G_{f p_{2} b p_{2}}\right]} \\
{\left[G_{t p_{2} f p_{2}}\right]} & {\left[G_{t p_{2} t p_{2}}\right]} & {\left[G_{t p_{2} b p_{2}}\right]} \\
{\left[G_{b p_{2} f p_{2}}\right]} & {\left[G_{b p_{2} t p_{2}}\right]} & {\left[G_{b p_{2} b p_{2}}\right]}
\end{array}\right] \cdot\left\{\begin{array}{l}
P_{f p_{2}} \\
P_{t p_{2}} \\
P_{b p_{2}}
\end{array}\right\}
$$

Isolando as forças de superfície de (3.33.1):

$P_{f p_{2}}=\left[G_{f p_{2} f p_{2}}\right]^{-1} \cdot\left\{\left[H_{f p_{2} f p_{2}}\right] \cdot U_{f p_{2}}+\left[H_{f p_{2} t p_{2}}\right] \cdot U_{t p_{2}}+\left[H_{f p_{2} b p_{2}}\right] \cdot U_{b p_{2}}-\left[G_{f p_{2} t p_{2}}\right] \cdot P_{t p_{2}}-\left[G_{f p_{2} b p_{2}}\right] \cdot P_{b p_{2}}\right\}$ 
Aplicando as condições de equilíbrio e compatibilidade para o fuste:

$$
\begin{aligned}
& P_{f s_{1}}=-P_{f p_{1}} \\
& P_{f s_{2}}=-P_{f p_{2}} \\
& U_{f s_{1}}=U_{f p_{1}} \\
& U_{f s_{2}}=U_{f p_{2}}
\end{aligned}
$$

Usando-se as relações de (3.35.1) a (3.35.4), já se levando em consideração (3.32) e (3.34) nas expressões de (3.30.1) a (3.30.4), tem-se então:

$$
\begin{aligned}
& {\left[H_{t s t s}\right] \cdot U_{t s}+\left[H_{t s b s}\right] \cdot U_{b s}+\left\{\left[H_{t s f s_{1}}\right]+\left[G_{t s f_{1}}\right] \cdot\left[G_{f f p_{1} f p_{1}}\right]^{-1} \cdot\left[H_{f p_{1} f p_{1}}\right]\right\} \cdot U_{f s_{1}}+} \\
& \left\{\left[H_{t s f_{2}}\right]+\left[G_{t s s_{2}}\right] \cdot\left[G_{f p_{2} f p_{2}}\right]^{-1} \cdot\left[H_{f p_{2} f p_{2}}\right]\right\} \cdot U_{f s_{2}}+\left\{\left[G_{t s f_{1}}\right] \cdot\left[G_{f p_{1} f p_{1}}\right]^{-1} \cdot\left[H_{f p_{1} t p_{1}}\right]\right\} \cdot U_{t p_{1}} \\
& +\left\{\left[G_{t s \delta_{1}}\right] \cdot\left[G_{f p_{1} f p_{1}}\right]^{-1} \cdot\left[H_{f p_{1} b p_{1}}\right]\right\} \cdot U_{b p_{1}}+\left\{\left[G_{t s s_{2}}\right] \cdot\left[G_{f p_{2} f p_{2}}\right]^{-1} \cdot\left[H_{f p_{2} t p_{2}}\right]\right\} \cdot U_{t p_{2}}+ \\
& \left\{\left[G_{t s \delta_{2}}\right] \cdot\left[G_{f p_{2} f p_{2}}\right]^{-1} \cdot\left[H_{f p_{2} b p_{2}}\right]\right\} \cdot U_{b p_{2}}=\left[G_{t s t s}\right] \cdot P_{t s}+\left[G_{t s b s}\right] \cdot U_{b s}+ \\
& \left\{\left[G_{t s f_{1}}\right] \cdot\left[G_{f p_{1}, f p_{1}}\right]^{-1} \cdot\left[G_{f p_{1} t p_{1}}\right]\right\} \cdot P_{t p_{1}}+\left\{\left[G_{t s f_{1}}\right] \cdot\left[G_{f p_{1} f f_{1}}\right]^{-1} \cdot\left[G_{f p_{1} b p_{1}}\right]\right\} \cdot P_{b p_{1}}+ \\
& \left\{\left[G_{t s f_{2}}\right] \cdot\left[G_{f p_{2} f p_{2}}\right]^{-1} \cdot\left[G_{f p_{2} t p_{2}}\right]\right\} \cdot P_{t p_{2}}+\left\{\left[G_{t s f_{2}}\right] \cdot\left[G_{f f p_{2} f p_{2}}\right]^{-1} \cdot\left[G_{f f p_{2} b p_{2}}\right]\right\} \cdot P_{b p_{2}}
\end{aligned}
$$

$$
\begin{aligned}
& {\left[H_{b s t s}\right] \cdot U_{t s}+\left[H_{b s s s}\right] \cdot U_{b s}+\left\{\left[H_{b s f f_{1}}\right]+\left[G_{b s f f_{1}}\right] \cdot\left[G_{f p_{1} f p_{1}}\right]^{-1} \cdot\left[H_{f p_{1}, f p_{1}}\right]\right\} \cdot U_{f s_{1}}+} \\
& \left\{\left[H_{b s f s_{2}}\right]+\left[G_{b s f s_{2}}\right] \cdot\left[G_{f f p_{2} f p_{2}}\right]^{-1} \cdot\left[H_{f p_{2} f p_{2}}\right]\right\} \cdot U_{f s_{2}}+\left\{\left[G_{b s f s_{1}}\right] \cdot\left[G_{f p_{1} f p_{1}}\right]^{-1} \cdot\left[H_{f p_{1} t_{1}}\right]\right\} \cdot U_{t p_{1}}+ \\
& \left\{\left[G_{b s \mathcal{S}_{1}}\right] \cdot\left[G_{f p_{1} f f_{1}}\right]^{-1} \cdot\left[H_{f p_{1} b p_{1}}\right]\right\} \cdot U_{b p_{1}}+\left\{\left[G_{b s s_{2}}\right] \cdot\left[G_{f p_{2} f p_{2}}\right]^{-1} \cdot\left[H_{f p_{2} p_{2}}\right]\right\} \cdot U_{t p_{2}}+ \\
& \left\{\left[G_{b s f_{2}}\right] \cdot\left[G_{f p_{2} f p_{2}}\right]^{-1} \cdot\left[H_{f p_{2} b p_{2}}\right]\right\} \cdot U_{b p_{2}}=\left[G_{b s t s}\right] \cdot P_{t s}+\left[G_{b s b s}\right] \cdot U_{b s}+ \\
& \left\{\left[G_{b s s_{1}}\right] \cdot\left[G_{f p_{1} f p_{1}}\right]^{-1} \cdot\left[G_{f p_{1} t_{1}}\right]\right\} \cdot P_{t p_{1}}+\left\{\left[G_{b s s_{1}}\right] \cdot\left[G_{f p_{1} f p_{1}}\right]^{-1} \cdot\left[G_{f p_{1} b p_{1}}\right]\right\} \cdot P_{b p_{1}}+ \\
& \left\{\left[G_{b s \delta_{2}}\right] \cdot\left[G_{f p_{2} f p_{2}}\right]^{-1} \cdot\left[G_{f p_{2} t p_{2}}\right]\right\} \cdot P_{t p_{2}}+\left\{\left[G_{b s s_{2}}\right] \cdot\left[G_{f p_{2} f p_{2}}\right]^{-1} \cdot\left[G_{f p_{2} b p_{2}}\right]\right\} \cdot P_{b p_{2}}
\end{aligned}
$$




$$
\begin{aligned}
& {\left[H_{f s_{1}, s}\right] \cdot U_{t s}+\left[H_{f s_{1} b s}\right] \cdot U_{b s}+\left\{\left[H_{f s_{1}, f_{1}}\right]+\left[G_{f s_{1}, f_{1}}\right] \cdot\left[G_{f p_{1}, f_{1}}\right]^{-1} \cdot\left[H_{f p_{1}, f_{1}}\right]\right\} \cdot U_{f f_{1}}+} \\
& \left\{\left[H_{f s_{1}, f_{2}}\right]+\left[G_{f f_{1}, s_{2}}\right] \cdot\left[G_{f p_{2} f p_{2}}\right]^{-1} \cdot\left[H_{f p_{2} f p_{2}}\right]\right\} \cdot U_{f s_{2}}+\left\{\left[G_{f s_{1}, f_{1}}\right] \cdot\left[G_{f p_{1} f p_{1}}\right]^{-1} \cdot\left[H_{f p_{1} t p_{1}}\right]\right\} \cdot U_{t p_{1}}+ \\
& \left\{\left[G_{f f_{1}, f f_{1}}\right] \cdot\left[G_{f p_{1} f p_{1}}\right]^{-1} \cdot\left[H_{f p_{1} b p_{1}}\right]\right\} \cdot U_{b p_{1}}+\left\{\left[G_{f s_{1}, s_{2}}\right] \cdot\left[G_{f p_{2} f p_{2}}\right]^{-1} \cdot\left[H_{f p_{2} t p_{2}}\right]\right\} \cdot U_{t p_{2}}+ \\
& \left\{\left[G_{f s_{1}, f s_{2}}\right] \cdot\left[G_{f p_{2} f p_{2}}\right]^{-1} \cdot\left[H_{f p_{2} b p_{2}}\right]\right\} \cdot U_{b p_{2}}=\left[G_{f s_{1} t s}\right] \cdot P_{t s}+\left[G_{f s_{s} b s}\right] \cdot U_{b s}+ \\
& \left\{\left[G_{f s_{1}, s_{1}}\right] \cdot\left[G_{f p_{1}, f p_{1}}\right]^{-1} \cdot\left[G_{f p_{1} t_{1}}\right]\right\} \cdot P_{t p_{1}}+\left\{\left[G_{f s_{1}, s_{1}}\right] \cdot\left[G_{f p_{1}, f p_{1}}\right]^{-1} \cdot\left[G_{f p_{1}, b_{1}}\right]\right\} \cdot P_{b p_{1}}+ \\
& \left\{\left[G_{f s_{1}, s_{2}}\right] \cdot\left[G_{f p_{2}, f p_{2}}\right]^{-1} \cdot\left[G_{f p_{2} t p_{2}}\right]\right\} \cdot P_{t p_{2}}+\left\{\left[G_{f s_{1}, s_{2}}\right] \cdot\left[G_{f p_{2} f p_{2}}\right]^{-1} \cdot\left[G_{f p_{2} b p_{2}}\right]\right\} \cdot P_{b p_{2}}
\end{aligned}
$$

$$
\begin{aligned}
& {\left[H_{f s_{2} t s}\right] \cdot U_{t s}+\left[H_{f s_{2} b s}\right] \cdot U_{b s}+\left\{\left[H_{f s_{2} f s_{1}}\right]+\left[G_{f s_{2} f f_{1}}\right] \cdot\left[G_{f p_{1} f p_{1}}\right]^{-1} \cdot\left[H_{f p_{1} f p_{1}}\right]\right\} \cdot U_{f s_{1}}+} \\
& \left\{\left[H_{f s_{2}, f_{2}}\right]+\left[G_{f s_{2}, f_{2}}\right] \cdot\left[G_{f p_{2} f p_{2}}\right]^{-1} \cdot\left[H_{f p_{2} f p_{2}}\right]\right\} \cdot U_{f s_{2}}+\left\{\left[G_{f s_{2}, f_{1}}\right] \cdot\left[G_{f p_{1} f p_{1}}\right]^{-1} \cdot\left[H_{f p_{1} t p_{1}}\right]\right\} \cdot U_{t p_{1}}+ \\
& \left\{\left[G_{f f_{2} f_{1}}\right] \cdot\left[G_{f p_{1}, f p_{1}}\right]^{-1} \cdot\left[H_{f p_{1} b p_{1}}\right]\right\} \cdot U_{b p_{1}}+\left\{\left[G_{f f_{2}, s_{2}}\right] \cdot\left[G_{f p_{2} f f_{2}}\right]^{-1} \cdot\left[H_{f p_{2} t p_{2}}\right]\right\} \cdot U_{t p_{2}}+ \\
& \left\{\left[G_{f f_{2} f_{2}}\right] \cdot\left[G_{f p_{2} f p_{2}}\right]^{-1} \cdot\left[H_{f p_{2} b p_{2}}\right]\right\} \cdot U_{b p_{2}}=\left[G_{f f_{2} t s}\right] \cdot P_{t s}+\left[G_{f f_{2} b s}\right] \cdot U_{b s}+ \\
& \left\{\left[G_{f s_{2}, f_{1}}\right] \cdot\left[G_{f p_{1} f p_{1}}\right]^{-1} \cdot\left[G_{f p_{1} t p_{1}}\right]\right\} \cdot P_{t p_{1}}+\left\{\left[G_{f s_{2}, f_{1}}\right] \cdot\left[G_{f p_{1} f p_{1}}\right]^{-1} \cdot\left[G_{f p_{1} b p_{1}}\right]\right\} \cdot P_{b p_{1}}+ \\
& \left\{\left[G_{f s_{2}, f_{2}}\right] \cdot\left[G_{f p_{2}, f p_{2}}\right]^{-1} \cdot\left[G_{f p_{2} t p_{2}}\right]\right\} \cdot P_{t p_{2}}+\left\{\left[G_{f s_{2}, f_{2}}\right] \cdot\left[G_{f p_{2} f p_{2}}\right]^{-1} \cdot\left[G_{f p_{2} b p_{2}}\right]\right\} \cdot P_{b p_{2}}
\end{aligned}
$$

Usando-se as relações de (3.35.1) a (3.35.4) já se levando em consideração (3.32), nas expressões da estaca 1 (3.31.2) e (3.31.3), tem-se então:

$\left\lfloor\left[H_{t p_{1} t p_{1}}\right]-\left[G_{t p_{1}, p_{1}}\right] \cdot\left[G_{f p_{1}, f p_{1}}\right]^{-1} \cdot\left[H_{f p_{1} t p_{1}}\right]\right\rfloor \cdot U_{t p_{1}}+\left\lfloor\left[H_{t p_{1} b p_{1}}\right]-\left[G_{t p_{1}, f_{1}}\right] \cdot\left[G_{f p_{1}, f_{1}}\right]^{-1} \cdot\left[H_{f p_{1} b p_{1}}\right]\right\rfloor \cdot U_{b p_{1}}$ $+\left[\left[H_{t p_{1}, f_{p_{1}}}\right]-\left[G_{t p_{1}, f p_{1}}\right] \cdot\left[G_{f p_{1}, f p_{1}}\right]^{-1} \cdot\left[H_{f p_{1}, f_{p_{1}}}\right]\right] \cdot U_{f p_{1}}=$ $\left[\left[G_{t p_{1} p_{1}}\right]-\left[G_{t p_{1}, f_{1}}\right] \cdot\left[G_{f p_{1}, f f_{1}}\right]^{-1} \cdot\left[G_{f p_{1} t_{1}}\right]\right] \cdot P_{t p_{1}}+\left[\left[G_{t p_{1} b p_{1}}\right]-\left[G_{t p_{1}, f p_{1}}\right] \cdot\left[G_{f p_{1}, f p_{1}}\right]^{-1} \cdot\left[G_{f p_{1}, b_{1}}\right]\right] \cdot P_{b p_{1}}$

$\left\lfloor\left[H_{b p_{1} t p_{1}}\right]-\left[G_{b p_{1}, f p_{1}}\right] \cdot\left[G_{f p_{1} f p_{1}}\right]^{-1} \cdot\left[H_{f p_{1} t p_{1}}\right]\right\rfloor \cdot U_{t p_{1}}+\left\lfloor\left[H_{b p_{1} b p_{1}}\right]-\left[G_{b p_{1} f p_{1}}\right] \cdot\left[G_{f p_{1}, f p_{1}}\right]^{-1} \cdot\left[H_{f p_{1} b p_{1}}\right]\right\rfloor \cdot U_{b p_{1}}$ $+\left[\left[H_{b p_{1}, f_{1}}\right]-\left[G_{b p_{1}, f p_{1}}\right] \cdot\left[G_{f p_{1} f p_{1}}\right]^{-1} \cdot\left[H_{f p_{1}, f f_{1}}\right]\right] \cdot U_{f p_{1}}=$ $\left[\left[G_{b p_{1} t p_{1}}\right]-\left[G_{b p_{1}, f f_{1}}\right] \cdot\left[G_{f p_{1}, f p_{1}}\right]^{-1} \cdot\left[G_{f p_{1}, t_{1}}\right]\right] \cdot P_{t p_{1}}+\left[\left[G_{b p_{1}, p_{1}}\right]-\left[G_{b p_{1}, f p_{1}}\right] \cdot\left[G_{f p_{1} f p_{1}}\right]^{-1} \cdot\left[G_{f p_{1} b p_{1}}\right]\right] \cdot P_{b p_{1}}$

\section{Da mesma forma para a estaca 2:}




$$
\begin{aligned}
& \left\lfloor\left[H_{t p_{2} t p_{2}}\right]-\left[G_{t p_{2} f p_{2}}\right] \cdot\left[G_{f p_{2} f p_{2}}\right]^{-1} \cdot\left[H_{f p_{2} t p_{2}}\right]\right] \cdot U_{t p_{2}}+\left\lfloor\left[H_{t p_{2} b p_{2}}\right]-\left[G_{t p_{2} f p_{2}}\right] \cdot\left[G_{f p_{2} f p_{2}}\right]^{-1} \cdot\left[H_{f p_{2} b p_{2}}\right]\right] \cdot U_{b p_{2}} \\
& +\left[\left[H_{t p_{2} f p_{2}}\right]-\left[G_{t p_{2} f p_{2}}\right] \cdot\left[G_{f p_{2} f p_{2}}\right]^{-1} \cdot\left[H_{f p_{2} f p_{2}}\right]\right] \cdot U_{f p_{2}}= \\
& {\left[\left[G_{t p_{2} t p_{2}}\right]-\left[G_{t p_{2} f p_{2}}\right] \cdot\left[G_{f p_{2} f p_{2}}\right]^{-1} \cdot\left[G_{f p_{2} t p_{2}}\right]\right] \cdot P_{t p_{2}}+\left[\left[G_{t p_{2} b p_{2}}\right]-\left[G_{t p_{2} f p_{2}}\right] \cdot\left[G_{f p_{2} f p_{2}}\right]^{-1} \cdot\left[G_{f p_{2} b p_{2}}\right]\right] \cdot P_{b p_{2}}}
\end{aligned}
$$

$$
\begin{aligned}
& \left\lfloor\left[H_{b p_{2} t p_{2}}\right]-\left[G_{b p_{2} f p_{2}}\right] \cdot\left[G_{f p_{2} f p_{2}}\right]^{-1} \cdot\left[H_{f p_{2} t p_{2}}\right]\right] \cdot U_{t p_{2}}+\left\lfloor\left[H_{b p_{2} b p_{2}}\right]-\left[G_{b p_{2} f p_{2}}\right] \cdot\left[G_{f p_{2} f p_{2}}\right]^{-1} \cdot\left[H_{f p_{2} b p_{2}}\right]\right] \cdot U_{b p_{2}} \\
& +\left[\left[H_{b p_{2} f p_{2}}\right]-\left[G_{b p_{2} f p_{2}}\right] \cdot\left[G_{f p_{2} f p_{2}}\right]^{-1} \cdot\left[H_{f p_{2} f p_{2}}\right]\right] \cdot U_{f p_{2}} \\
& {\left[\left[G_{b p_{2} t p_{2}}\right]-\left[G_{b p_{2} f p_{2}}\right] \cdot\left[G_{f p_{2} f p_{2}}\right]^{-1} \cdot\left[G_{f p_{2} t p_{2}}\right]\right] \cdot P_{t p_{2}}+\left[\left[G_{b p_{2} b p_{2}}\right]-\left[G_{b p_{2} f p_{2}}\right] \cdot\left[G_{f p_{2} f p_{2}}\right]^{-1} \cdot\left[G_{f p_{2} b p_{2}}\right]\right] \cdot P_{b p_{2}}}
\end{aligned}
$$

\section{Nomeando as submatrizes da seguinte forma:}

$$
\begin{aligned}
& H_{11}=\left[H_{t s t s}\right] \\
& H_{12}=\left[H_{t s b s}\right] \\
& H_{13}=\left[G_{t s f s_{1}}\right] \cdot\left[G_{f p_{1} f p_{1}}\right]^{-1} \cdot\left[H_{f p_{1} t p_{1}}\right] \\
& H_{14}=\left[G_{t s f s_{1}}\right] \cdot\left[G_{f p_{1} f p_{1}}\right]^{-1} \cdot\left[H_{f p_{1} b p_{1}}\right] \\
& H_{15}=\left[G_{t s f s_{2}}\right] \cdot\left[G_{f p_{2} f p_{2}}\right]^{-1} \cdot\left[H_{f p_{2} t p_{2}}\right] \\
& H_{16}=\left[G_{t s f s_{2}}\right] \cdot\left[G_{f p_{2} f p_{2}}\right]^{-1} \cdot\left[H_{f p_{2} b p_{2}}\right] \\
& H_{17}=\left[H_{t s f_{1}}\right]+\left[G_{t s f_{1}}\right] \cdot\left[G_{f p_{1} f p_{1}}\right]^{-1} \cdot\left[H_{f p_{1} f p_{1}}\right] \\
& H_{18}=\left[H_{t s f f_{2}}\right]+\left[G_{t s f_{2}}\right] \cdot\left[G_{f p_{2} f p_{2}}\right]^{-1} \cdot\left[H_{f p_{2} f p_{2}}\right] \\
& G_{11}=\left[G_{t s t s}\right] \\
& G_{12}=\left[G_{t s b s}\right] \\
& G_{13}=\left[G_{t s f_{1}}\right] \cdot\left[G_{f p_{1} f p_{1}}\right]^{-1} \cdot\left[G_{f p_{1} t p_{1}}\right] \\
& G_{14}=\left[G_{t s f_{1}}\right] \cdot\left[G_{f p_{1} f p_{1}}\right]^{-1} \cdot\left[G_{f p_{1} b p_{1}}\right] \\
& G_{15}=\left[G_{t s s_{2}}\right] \cdot\left[G_{f p_{2} f p_{2}}\right]^{-1} \cdot\left[G_{f p_{2} t p_{2}}\right] \\
& G_{16}=\left[G_{t s f_{2}}\right] \cdot\left[G_{f p_{2} f p_{2}}\right]^{-1} \cdot\left[G_{f p_{2} b p_{2}}\right]
\end{aligned}
$$




$$
\begin{aligned}
& H_{21}=\left[H_{b s t s}\right] \\
& H_{22}=\left[H_{b s b s}\right] \\
& H_{23}=\left[G_{b s f f_{1}}\right] \cdot\left[G_{f p_{1} f p_{1}}\right]^{-1} \cdot\left[H_{f p_{1} t p_{1}}\right] \\
& H_{24}=\left[G_{b s f s_{1}}\right] \cdot\left[G_{f p_{1} f p_{1}}\right]^{-1} \cdot\left[H_{f p_{1} b p_{1}}\right] \\
& H_{25}=\left[G_{b s f s_{2}}\right] \cdot\left[G_{f p_{2} f p_{2}}\right]^{-1} \cdot\left[H_{f p_{2} t p_{2}}\right] \\
& H_{26}=\left[G_{b s f s_{2}}\right] \cdot\left[G_{f p_{2} f p_{2}}\right]^{-1} \cdot\left[H_{f p_{2} b p_{2}}\right] \\
& H_{27}=\left[H_{b s f f_{1}}\right]+\left[G_{b s f_{1}}\right] \cdot\left[G_{f p_{1} f p_{1}}\right]^{-1} \cdot\left[H_{f p_{1} f p_{1}}\right] \\
& H_{28}=\left[H_{b s f f_{2}}\right]+\left[G_{b s f s_{2}}\right] \cdot\left[G_{f p_{2} f p_{2}}\right]^{-1} \cdot\left[H_{f p_{2} f p_{2}}\right] \\
& G_{21}=\left[G_{b s t s}\right] \\
& G_{22}=\left[G_{b s b s}\right] \\
& G_{23}=\left[G_{b s f s_{1}}\right] \cdot\left[G_{f p_{1} f p_{1}}\right]^{-1} \cdot\left[G_{f p_{1} t p_{1}}\right] \\
& G_{24}=\left[G_{b s f s_{1}}\right] \cdot\left[G_{f p_{1} f p_{1}}\right]^{-1} \cdot\left[G_{f p_{1} b p_{1}}\right] \\
& G_{25}=\left[G_{b s f s_{2}}\right] \cdot\left[G_{f p_{2} f p_{2}}\right]^{-1} \cdot\left[G_{f p_{2} t p_{2}}\right] \\
& G_{26}=\left[G_{b s f s_{2}}\right] \cdot\left[G_{f p_{2} f p_{2}}\right]^{-1} \cdot\left[G_{f p_{2} b p_{2}}\right]
\end{aligned}
$$

$$
\begin{aligned}
& H_{33}=\left[H_{t p_{1} t p_{1}}\right]-\left[G_{t p_{1} f p_{1}}\right] \cdot\left[G_{f p_{1} f p_{1}}\right]^{-1} \cdot\left[H_{f p_{1} t p_{1}}\right] \\
& H_{34}=\left[H_{t p_{1} b p_{1}}\right]-\left[G_{t p_{1} f p_{1}}\right] \cdot\left[G_{f p_{1} f p_{1}}\right]^{-1} \cdot\left[H_{f p_{1} b p_{1}}\right] \\
& H_{37}=\left[H_{t p_{1} f p_{1}}\right]-\left[G_{t p_{1} f p_{1}}\right] \cdot\left[G_{f p_{1} f p_{1}}\right]^{-1} \cdot\left[H_{f p_{1} f p_{1}}\right] \\
& G_{33}=\left[G_{t p_{1} t p_{1}}\right]-\left[G_{t p_{1} f p_{1}}\right] \cdot\left[G_{f p_{1} f p_{1}}\right]^{-1} \cdot\left[G_{f p_{1} t p_{1}}\right] \\
& G_{34}=\left[G_{t p_{1} b p_{1}}\right]-\left[G_{t p_{1} f p_{1}}\right] \cdot\left[G_{f p_{1} f p_{1}}\right]^{-1} \cdot\left[G_{f p_{1} b p_{1}}\right]
\end{aligned}
$$

$$
\begin{aligned}
& H_{43}=\left[H_{b p_{1} t p_{1}}\right]-\left[G_{b p_{1} f p_{1}}\right] \cdot\left[G_{f p_{1} f p_{1}}\right]^{-1} \cdot\left[H_{f p_{1} t p_{1}}\right] \\
& H_{44}=\left[H_{b p_{1} b p_{1}}\right]-\left[G_{b p_{1} f p_{1}}\right] \cdot\left[G_{f p_{1} f p_{1}}\right]^{-1} \cdot\left[H_{f p_{1} b p_{1}}\right] \\
& H_{47}=\left[H_{b p_{1} f p_{1}}\right]-\left[G_{b p_{1} f p_{1}}\right] \cdot\left[G_{f p_{1} f p_{1}}\right]^{-1} \cdot\left[H_{f p_{1} f p_{1}}\right] \\
& G_{43}=\left[G_{b p_{1} t p_{1}}\right]-\left[G_{b p_{1} f p_{1}}\right] \cdot\left[G_{f p_{1} f p_{1}}\right]^{-1} \cdot\left[G_{f p_{1} t p_{1}}\right] \\
& G_{44}=\left[G_{b p_{1} b p_{1}}\right]-\left[G_{b p_{1} f p_{1}}\right] \cdot\left[G_{f p_{1} f p_{1}}\right]^{-1} \cdot\left[G_{f p_{1} b p_{1}}\right]
\end{aligned}
$$




$$
\begin{aligned}
& H_{55}=\left[H_{t p_{2} t p_{2}}\right]-\left[G_{t p_{2} f p_{2}}\right] \cdot\left[G_{f p_{2} f p_{2}}\right]^{-1} \cdot\left[H_{f p_{2} t p_{2}}\right] \\
& H_{56}=\left[H_{t p_{2} b p_{2}}\right]-\left[G_{t p_{2} f p_{2}}\right] \cdot\left[G_{f p_{2} f p_{2}}\right]^{-1} \cdot\left[H_{f p_{2} b p_{2}}\right] \\
& H_{58}=\left[H_{t p_{2} f p_{2}}\right]-\left[G_{t p_{2} f p_{2}}\right] \cdot\left[G_{f p_{2} f p_{2}}\right]^{-1} \cdot\left[H_{f p_{2} f p_{2}}\right] \\
& G_{55}=\left[G_{t p_{2} t p_{2}}\right]-\left[G_{t p_{2} f p_{2}}\right] \cdot\left[G_{f p_{2} f p_{2}}\right]^{-1} \cdot\left[G_{f p_{2} t p_{2}}\right] \\
& G_{56}=\left[G_{t p_{2} b p_{2}}\right]-\left[G_{t p_{2} f p_{2}}\right] \cdot\left[G_{f p_{2} f p_{2}}\right]^{-1} \cdot\left[G_{f p_{2} b p_{2}}\right]
\end{aligned}
$$

$$
\begin{aligned}
& H_{65}=\left[H_{b p_{2} t p_{2}}\right]-\left[G_{b p_{2} f p_{2}}\right] \cdot\left[G_{f p_{2} f p_{2}}\right]^{-1} \cdot\left[H_{f p_{2} t p_{2}}\right] \\
& H_{66}=\left[H_{b p_{2} b p_{2}}\right]-\left[G_{b p_{2} f p_{2}}\right] \cdot\left[G_{f p_{2} f p_{2}}\right]^{-1} \cdot\left[H_{f p_{2} b p_{2}}\right] \\
& H_{68}=\left[H_{b p_{2} f p_{2}}\right]-\left[G_{b p_{2} f p_{2}}\right] \cdot\left[G_{f p_{2} f p_{2}}\right]^{-1} \cdot\left[H_{f p_{2} f p_{2}}\right] \\
& G_{65}=\left[G_{b p_{2} t p_{2}}\right]-\left[G_{b p_{2} f p_{2}}\right] \cdot\left[G_{f p_{2} f p_{2}}\right]^{-1} \cdot\left[G_{f p_{2} t p_{2}}\right] \\
& G_{66}=\left[G_{b p_{2} b p_{2}}\right]-\left[G_{b p_{2} f p_{2}}\right] \cdot\left[G_{f p_{2} f p_{2}}\right]^{-1} \cdot\left[G_{f p_{2} b p_{2}}\right.
\end{aligned}
$$

$$
\begin{aligned}
& H_{71}=\left[H_{f_{s_{1} t s}}\right] \\
& H_{72}=\left[H_{f_{1} b s}\right] \\
& H_{73}=\left[G_{f s_{1} f f_{1}}\right] \cdot\left[G_{f p_{1} f p_{1}}\right]^{-1} \cdot\left[H_{f p_{1} t p_{1}}\right] \\
& H_{74}=\left[G_{f s_{1} f_{S_{1}}}\right] \cdot\left[G_{f p_{1} f p_{1}}\right]^{-1} \cdot\left[H_{f p_{1} b p_{1}}\right] \\
& H_{75}=\left[G_{f s_{1} f s_{2}}\right] \cdot\left[G_{f p_{2} f p_{2}}\right]^{-1} \cdot\left[H_{f p_{2} t p_{2}}\right] \\
& H_{76}=\left[G_{f s_{1} f s_{2}}\right] \cdot\left[G_{f p_{2} f p_{2}}\right]^{-1} \cdot\left[H_{f p_{2} b p_{2}}\right] \\
& H_{77}=\left[H_{f s_{1} f_{s_{1}}}\right]+\left[G_{f s_{1} f s_{1}}\right] \cdot\left[G_{f p_{1} f p_{1}}\right]^{-1} \cdot\left[H_{f p_{1} f p_{1}}\right] \\
& H_{78}=\left[H_{f s_{1} f s_{2}}\right]+\left[G_{f s_{1} f s_{2}}\right] \cdot\left[G_{f p_{2} f p_{2}}\right]^{-1} \cdot\left[H_{f p_{2} f p_{2}}\right] \\
& G_{71}=\left[G_{f_{1} t s}\right] \\
& G_{72}=\left[G_{f_{1} b s}\right] \\
& G_{73}=\left[G_{f s_{1} f s_{1}}\right] \cdot\left[G_{f p_{1} f p_{1}}\right]^{-1} \cdot\left[G_{f p_{1} t p_{1}}\right] \\
& G_{74}=\left[G_{f s_{1} f f_{1}}\right] \cdot\left[G_{f p_{1} f p_{1}}\right]^{-1} \cdot\left[G_{f p_{1} b p_{1}}\right] \\
& G_{75}=\left[G_{f s_{1} f s_{2}}\right] \cdot\left[G_{f p_{2} f p_{2}}\right]^{-1} \cdot\left[G_{f p_{2} t p_{2}}\right] \\
& G_{76}=\left[G_{f s_{1}, s_{2}}\right] \cdot\left[G_{f p_{2} f p_{2}}\right]^{-1} \cdot\left[G_{f p_{2} b p_{2}}\right]
\end{aligned}
$$




$$
\begin{aligned}
& H_{81}=\left[H_{f s_{2} t s}\right] \\
& H_{82}=\left[H_{f s_{2} b s}\right] \\
& H_{83}=\left[G_{f s_{2} f s_{1}}\right] \cdot\left[G_{f p_{1} f p_{1}}\right]^{-1} \cdot\left[H_{f p_{1} t p_{1}}\right] \\
& H_{84}=\left[G_{f s_{2} f s_{1}}\right] \cdot\left[G_{f p_{1} f p_{1}}\right]^{-1} \cdot\left[H_{f p_{1} b p_{1}}\right] \\
& H_{85}=\left[G_{f s_{2} f s_{2}}\right] \cdot\left[G_{f p_{2} f p_{2}}\right]^{-1} \cdot\left[H_{f p_{2} t p_{2}}\right] \\
& H_{86}=\left[G_{f s_{2} f s_{2}}\right] \cdot\left[G_{f p_{2} f p_{2}}\right]^{-1} \cdot\left[H_{f p_{2} b p_{2}}\right] \\
& H_{87}=\left[H_{f s_{2} f s_{1}}\right]+\left[G_{f s_{2} f s_{1}}\right] \cdot\left[G_{f p_{1} f p_{1}}\right]^{-1} \cdot\left[H_{f p_{1} f p_{1}}\right] \\
& H_{88}=\left[H_{f s_{2} f s_{2}}\right]+\left[G_{f s_{2} f s_{2}}\right] \cdot\left[G_{f p_{2} f p_{2}}\right]^{-1} \cdot\left[H_{f p_{2} f p_{2}}\right] \\
& G_{81}=\left[G_{f s_{2} t s}\right] \\
& G_{82}=\left[G_{f s_{2} b s}\right] \\
& G_{83}=\left[G_{f s_{2} f s_{1}}\right] \cdot\left[G_{f p_{1} f p_{1}}\right]^{-1} \cdot\left[G_{f p_{1} t p_{1}}\right] \\
& G_{84}=\left[G_{f s_{2} f s_{1}}\right] \cdot\left[G_{f p_{1} f p_{1}}\right]^{-1} \cdot\left[G_{f p_{1} b p_{1}}\right] \\
& G_{85}=\left[G_{f s_{2} f s_{2}}\right] \cdot\left[G_{f p_{2} f p_{2}}\right]^{-1} \cdot\left[G_{f p_{2} t p_{2}}\right] \\
& G_{86}=\left[G_{f s_{2} f f_{2}}\right] \cdot\left[G_{f p_{2} f p_{2}}\right]^{-1} \cdot\left[G_{f p_{2} b p_{2}}\right]
\end{aligned}
$$

Representando-se matricialmente o acoplamento estrato-estaca, onde as expressões das submatrizes de $[H]$ e [G] são dadas pelas relações de 3.44.1 a 3.51.14, tem-se: 


$$
\begin{aligned}
& {\left[\begin{array}{cccccccc}
H_{11} & H_{12} & H_{13} & H_{14} & H_{15} & H_{16} & H_{17} & H_{18} \\
H_{21} & H_{22} & H_{23} & H_{24} & H_{25} & H_{26} & H_{27} & H_{28} \\
0 & 0 & H_{33} & H_{34} & 0 & 0 & H_{37} & 0 \\
0 & 0 & H_{43} & H_{44} & 0 & 0 & H_{47} & 0 \\
0 & 0 & 0 & 0 & H_{55} & H_{56} & 0 & H_{58} \\
0 & 0 & 0 & 0 & H_{65} & H_{66} & 0 & H_{68} \\
H_{71} & H_{72} & H_{73} & H_{74} & H_{75} & H_{76} & H_{77} & H_{78} \\
H_{81} & H_{82} & H_{83} & H_{84} & H_{85} & H_{86} & H_{87} & H_{88}
\end{array}\right] \cdot\left\{\begin{array}{l}
U_{t s} \\
U_{b s} \\
U_{t p_{1}} \\
U_{b p_{1}} \\
U_{t p_{2}} \\
U_{b p_{2}} \\
U_{f s_{1}} \\
U_{f s_{2}}
\end{array}\right\}=} \\
& {\left[\begin{array}{ccccccc}
G_{11} & G_{12} & G_{13} & G_{14} & G_{15} & G_{16} \\
G_{21} & G_{22} & G_{23} & G_{24} & G_{25} & G_{26} \\
0 & 0 & G_{33} & G_{34} & 0 & 0 \\
0 & 0 & G_{43} & G_{44} & 0 & 0 \\
0 & 0 & 0 & 0 & G_{55} & G_{56} \\
0 & 0 & 0 & 0 & G_{65} & G_{66} \\
G_{71} & G_{72} & G_{73} & G_{74} & G_{75} & G_{76} \\
G_{81} & G_{82} & G_{83} & G_{84} & G_{85} & G_{86}
\end{array}\right] \cdot\left\{\begin{array}{l}
P_{t s} \\
P_{b s} \\
P_{t p_{1}} \\
P_{b p_{1}} \\
P_{t p_{2}} \\
P_{b p_{2}}
\end{array}\right\}}
\end{aligned}
$$

Agora, para um sistema com $\eta$ estacas, as submatrizes ficam para uma estaca genérica "i" em um estrato qualquer:

$$
\begin{aligned}
& H_{11}=\left[H_{t s t s}\right] \\
& H_{12}=\left[H_{t s b s}\right] \\
& H_{1(2 i+1)}=\left[G_{t s f_{i}}\right] \cdot\left[G_{f p_{i} f p_{i}}\right]^{-1} \cdot\left[H_{f p_{i} t p_{i}}\right] \\
& H_{1(2 i+2)}=\left[G_{t s f_{i}}\right] \cdot\left[G_{f p_{i} f p_{i}}\right]^{-1} \cdot\left[H_{f p_{i} b p_{i}}\right] \\
& H_{1(2 \eta+2+i)}=\left[H_{t s f_{i}}\right]+\left[G_{t s f_{i}}\right] \cdot\left[G_{f p_{i} f p_{i}}\right]^{-1} \cdot\left[H_{f p_{i} f p_{i}}\right] \\
& G_{11}=\left[G_{t s t s}\right] \\
& G_{12}=\left[G_{t s b s}\right] \\
& G_{1(2 i+1)}=\left[G_{t s s_{i}}\right] \cdot\left[G_{f p_{i} f p_{i}}\right]^{-1} \cdot\left[G_{f p_{i} t p_{i}}\right] \\
& G_{1(2 i+2)}=\left[G_{t s f_{i}}\right] \cdot\left[G_{f p_{i} f p_{i}}\right]^{-1} \cdot\left[G_{f p_{i} b p_{i}}\right]
\end{aligned}
$$




$$
\begin{aligned}
& H_{21}=\left[H_{b s t s}\right] \\
& H_{22}=\left[H_{b s b s}\right] \\
& H_{2(2 i+1)}=\left[G_{b s f s_{i}}\right] \cdot\left[G_{f p_{i} f p_{i}}\right]^{-1} \cdot\left[H_{f p_{i}, p_{i}}\right] \\
& H_{2(2 i+2)}=\left[G_{b s f_{i}}\right] \cdot\left[G_{f p_{i} f p_{i}}\right]^{-1} \cdot\left[H_{f p_{i} b p_{i}}\right] \\
& H_{2(2 \eta+2+i)}=\left[H_{b s s s_{i}}\right]+\left[G_{b s f s_{i}}\right] \cdot\left[G_{f p_{i} f p_{i}}\right]^{-1} \cdot\left[H_{f p_{i} f p_{i}}\right] \\
& G_{21}=\left[G_{b s t s}\right] \\
& G_{22}=\left[G_{b s b s}\right] \\
& G_{2(2 i+1)}=\left[G_{b s f_{i}}\right] \cdot\left[G_{f p_{i} f p_{i}}\right]^{-1} \cdot\left[G_{f p_{i} t p_{i}}\right] \\
& G_{2(2 i+2)}=\left[G_{b s f s_{i}}\right] \cdot\left[G_{f p_{i} f p_{i}}\right]^{-1} \cdot\left[G_{f p_{i} b p_{i}}\right]
\end{aligned}
$$

$$
\begin{aligned}
& H_{(2 i+1)(2 i+1)}=\left[H_{t p_{i} t p_{i}}\right]-\left[G_{t p_{i} f p_{i}}\right] \cdot\left[G_{f p_{i} f p_{i}}\right]^{-1} \cdot\left[H_{f p_{i} t p_{i}}\right] \\
& H_{(2 i+1)(2 i+2)}=\left[H_{t p_{i} b p_{i}}\right]-\left[G_{t p_{i} f p_{i}}\right] \cdot\left[G_{f p_{i} f p_{i}}\right]^{-1} \cdot\left[H_{f p_{i} b p_{i}}\right] \\
& H_{(2 i+1)(2 \eta+2+1)}=\left[H_{t p_{i} f p_{i}}\right]-\left[G_{t p_{i} f p_{i}}\right] \cdot\left[G_{f p_{i} f p_{i}}\right]^{-1} \cdot\left[H_{f p_{i} f p_{i}}\right] \\
& G_{(2 i+1)(2 i+1)}=\left[G_{t p_{i} t p_{i}}\right]-\left[G_{t p_{i} f p_{i}}\right] \cdot\left[G_{f p_{i} f p_{i}}\right]^{-1} \cdot\left[G_{f p_{i} t p_{i}}\right] \\
& G_{(2 i+1)(2 i+2)}=\left[G_{t p_{i} b p_{i}}\right]-\left[G_{t p_{i} f p_{i}}\right] \cdot\left[G_{f p_{i} f p_{i}}\right]^{-1} \cdot\left[G_{f p_{i} b p_{i}}\right]
\end{aligned}
$$

$$
\begin{aligned}
& H_{(2 i+2)(2 i+1)}=\left[H_{b p_{i} t p_{i}}\right]-\left[G_{b p_{i} f p_{i}}\right] \cdot\left[G_{f p_{i} f p_{i}}\right]^{-1} \cdot\left[H_{f p_{i} t p_{i}}\right] \\
& H_{(2 i+2)(2 i+2)}=\left[H_{b p_{i} b p_{i}}\right]-\left[G_{b p_{i} f p_{i}}\right] \cdot\left[G_{f p_{i} f p_{i}}\right]^{-1} \cdot\left[H_{f p_{i} b p_{i}}\right] \\
& H_{(2 i+2)(2 \eta+2+1)}=\left[H_{b p_{i} f p_{i}}\right]-\left[G_{b p_{i} f p_{i}}\right] \cdot\left[G_{f p_{i} f p_{i}}\right]^{-1} \cdot\left[H_{f p_{i} f p_{i}}\right] \\
& G_{(2 i+2)(2 i+1)}=\left[G_{b p_{i} t p_{i}}\right]-\left[G_{b p_{i} f p_{i}}\right] \cdot\left[G_{f p_{i} f p_{i}}\right]^{-1} \cdot\left[G_{f p_{i} t p_{i}}\right] \\
& G_{(2 i+2)(2 i+2)}=\left[G_{b p_{i} b p_{i}}\right]-\left[G_{b p_{i} f p_{i}}\right] \cdot\left[G_{f p_{i} f p_{i}}\right]^{-1} \cdot\left[G_{f p_{i} b p_{i}}\right]
\end{aligned}
$$

(3.56.1 a 3.56.5) 


$$
\begin{aligned}
& H_{(2 \eta+2+k) 1}=\left[H_{f s_{k} t s}\right] \\
& H_{(2 \eta+2+k) 2}=\left[H_{f s_{k} b s}\right] \\
& H_{(2 \eta+2+k)(2 i+1)}=\left[G_{f s_{k} f s_{i}}\right] \cdot\left[G_{f p_{i} f p_{i}}\right]^{-1} \cdot\left[H_{f p_{i} t p_{i}}\right] \\
& H_{(2 \eta+2+k)(2 i+2)}=\left[G_{f s_{k} f s_{i}}\right] \cdot\left[G_{f p_{i} f p_{i}}\right]^{-1} \cdot\left[H_{f p_{i} b p_{i}}\right] \\
& H_{(2 \eta+2+k)(2 \eta+1+k+i)}=\left[H_{f s_{k} f f_{i}}\right]+\left[G_{f s_{k} f s_{i}}\right] \cdot\left[G_{f p_{i} f p_{i}}\right]^{-1} \cdot\left[H_{f p_{i} f p_{i}}\right] \\
& G_{(2 \eta+2+k) 1}=\left[G_{f s_{k} t s}\right] \\
& G_{(2 \eta+2+k) 2}=\left[G_{f s_{k} b s}\right] \\
& G_{(2 \eta+2+k)(2 i+1)}=\left[G_{f s_{k} f f_{i}}\right] \cdot\left[G_{f p_{i} f p_{i}}\right]^{-1} \cdot\left[G_{f p_{i} t p_{i}}\right] \\
& G_{(2 \eta+2+k)(2 i+2)}=\left[G_{f s_{k} f s_{i}}\right] \cdot\left[G_{f p_{i} f p_{i}}\right]^{-1} \cdot\left[G_{f p_{i} b p_{i}}\right]
\end{aligned}
$$

Ou, posicionando-se esta estaca "i" no sistema estrato-estaca, tem-se então esquematicamente:
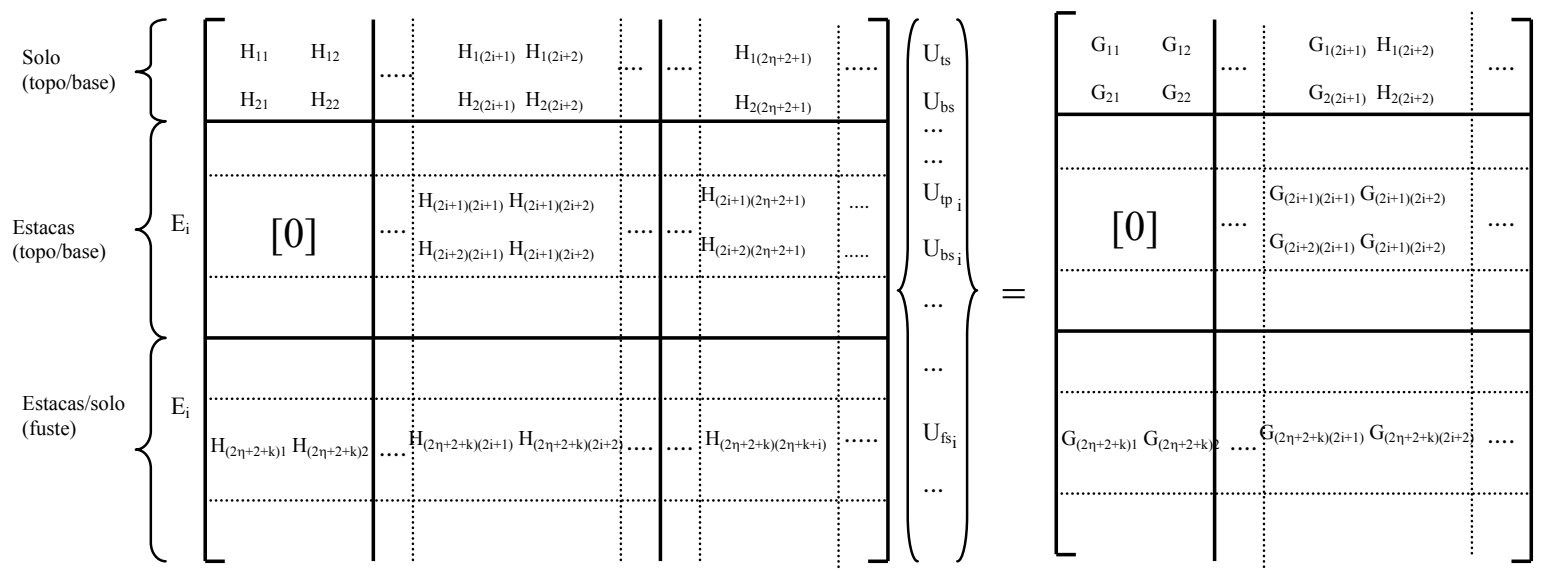

\subsubsection{Eliminação dos graus de liberdade do fuste}

O formato matricial do sistema (3.58) foi feito com o intuito de eliminar os graus de liberdade do fuste, deixando estas variáveis alocadas no final da matriz $[H]$. Desta forma transforma-se a expressão (3.58) em um sistema equivalente, eliminando-se, contudo, as variáveis do fuste. Esquematicamente, o sistema para uma camada genérica " $j$ " com " $\eta$ " estacas fica: 


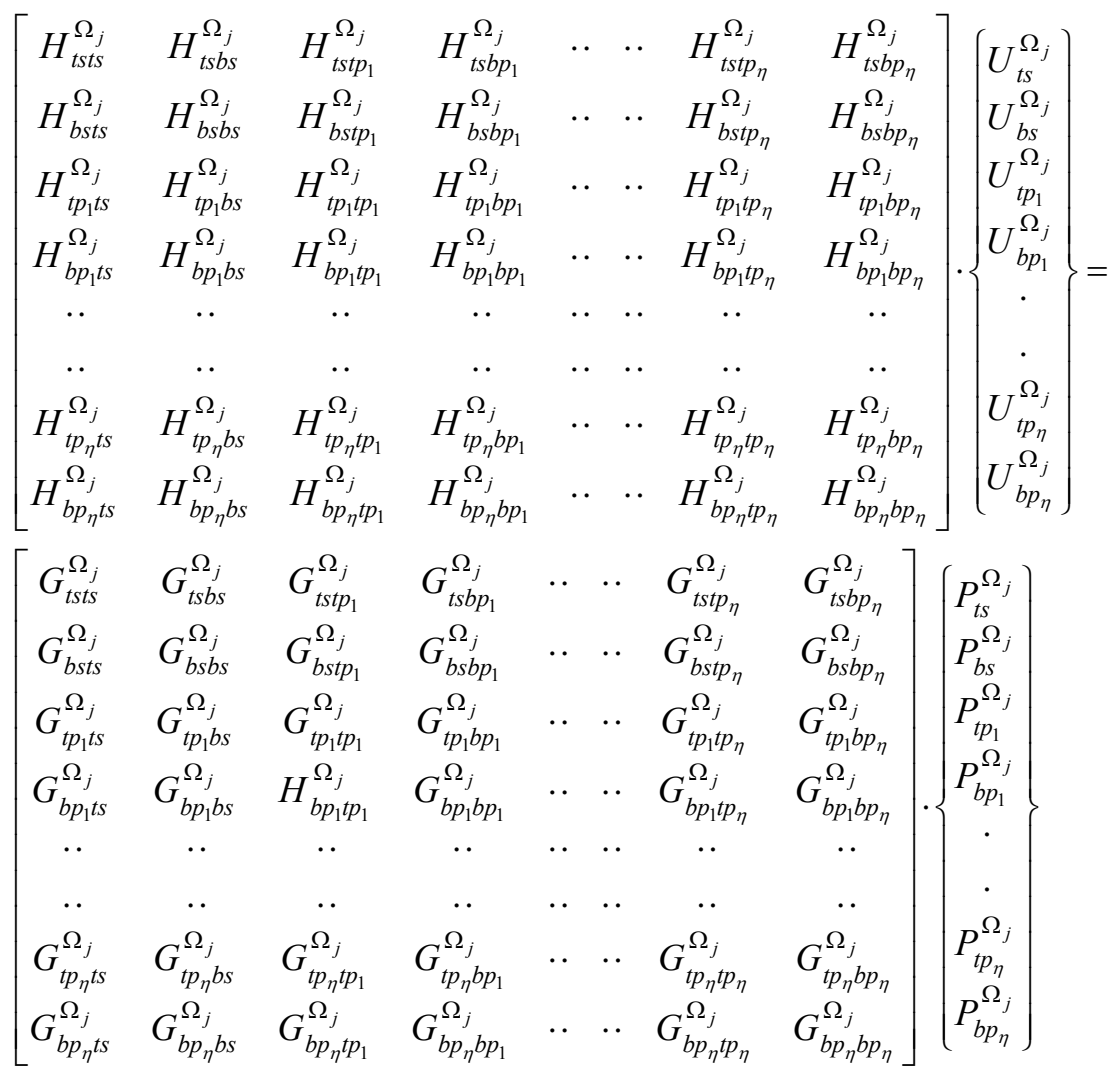

Para compatibilizar e equilibrar os estratos subseqüentes $j / j-1$ e $j / j+1$, é melhor escrever o sistema (3.59) da seguinte forma:

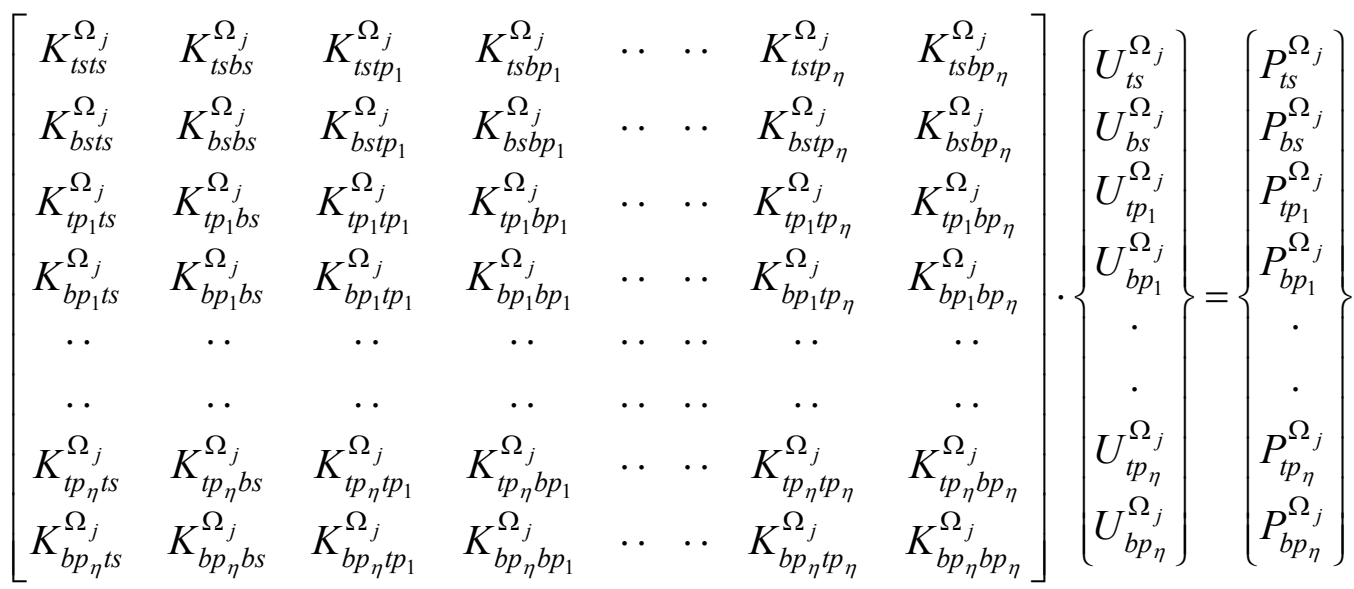

Chega-se à "matriz de rigidez" do estrato "j" que considera incorporadas " $\eta$ " estacas. Desta forma, podem-se compatibilizar e equilibrar as variáveis de deslocamentos e forças de contato entre as camadas adjacentes. 


\subsubsection{Compatibilidade e equilíbrio entre camadas}

A expressão (3.60) é a representação matricial do MEC já se levando em consideração as estacas imersas em uma camada genérica " $j$ " do solo. Para cada estrato, obtém-se este sistema, e as compatibilizações e os equilíbrios são feitos para as variáveis equivalentes. Este item tem o intuito de apresentar, de forma sistemática, como as camadas dos solos subseqüentes são associadas. Para isso, parte-se de um exemplo já com o número de estratos e de estacas determinados e mostra-se o procedimento realizado para obter os valores finais de deslocamentos da superfície livre. Pela figura 3.3, tem-se:

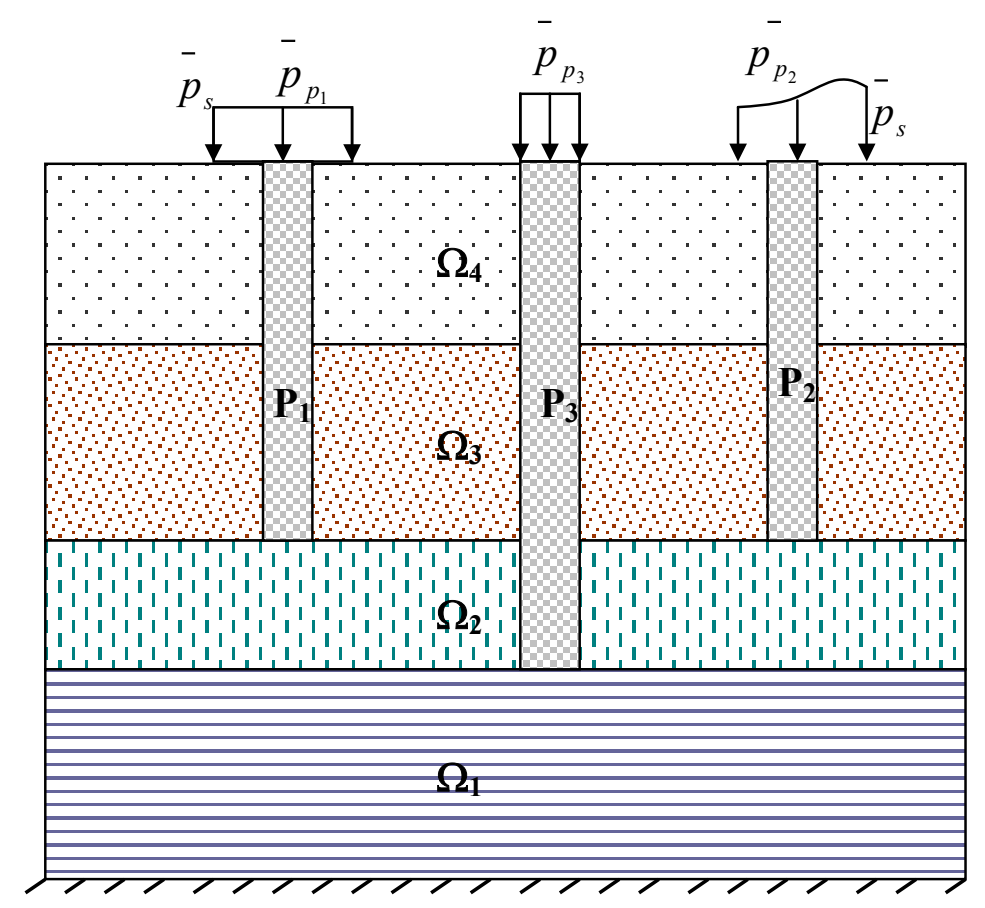

Figura 3.3 - Esquema do maciço estratificado estaqueado.

\section{$1^{a}$ camada:}

Como $\left\{U_{b}^{1}\right\}=\{0\}$, a expressão (3.60) para esta camada sem estacas fica: 


$$
\left[\begin{array}{cc}
K_{t s t s}^{\Omega_{1}} & K_{t s t p_{2}}^{\Omega_{1}} \\
K_{t p_{3} t s}^{\Omega_{1}} & K_{t p_{3} t p_{3}}^{\Omega_{1}}
\end{array}\right] \cdot\left\{\begin{array}{c}
U_{t s}^{\Omega_{1}} \\
U_{t p_{3}}^{\Omega_{1}}
\end{array}\right\}=\left\{\begin{array}{c}
P_{t s}^{\Omega_{1}} \\
P_{t p_{3}}^{\Omega_{1}}
\end{array}\right\}
$$

onde o subíndice "tpo" indica os valores do solo, mas que serão compatibilizados e equilibrados com a base da estaca 3 .

\section{$2^{a}$ camada:}

A equação (3.60) para esta camada fica:

$$
\left[\begin{array}{cccc}
K_{t s t s}^{\Omega_{2}} & K_{t s b s}^{\Omega_{2}} & K_{t s t p_{3}}^{\Omega_{2}} & K_{t s b p_{3}}^{\Omega_{2}} \\
K_{b s t s}^{\Omega_{2}} & K_{b s b s}^{\Omega_{2}} & K_{b s t p_{3}}^{\Omega_{2}} & K_{b s b p_{3}}^{\Omega_{2}} \\
K_{t p_{2} t s}^{\Omega_{2}} & K_{t p_{3} b s}^{\Omega_{2}} & K_{t p_{2} t p_{3}}^{\Omega_{2}} & K_{t p_{2} b p_{3}}^{\Omega_{2}} \\
K_{b p_{3} t s}^{\Omega_{2}} & K_{b p_{3} b s}^{\Omega_{2}} & K_{b p_{3} t p_{3}}^{\Omega_{2}} & K_{b p_{3} b p_{3}}^{\Omega_{2}}
\end{array}\right] \cdot\left\{\begin{array}{c}
U_{t s}^{\Omega_{2}} \\
U_{b s}^{\Omega_{2}} \\
U_{t p_{3}}^{\Omega_{2}} \\
U_{b p_{3}}^{\Omega_{2}}
\end{array}\right\}=\left\{\begin{array}{c}
P_{t s}^{\Omega_{2}} \\
P_{b s}^{\Omega_{2}} \\
P_{t p_{2}}^{\Omega_{2}} \\
P_{b p_{3}}^{\Omega_{2}}
\end{array}\right\}
$$

A segunda e a quarta expressão de (3.62) ficam:

$$
\begin{aligned}
& P_{b s}^{\Omega_{2}}=K_{b s t s}^{\Omega_{2}} \cdot U_{t s}^{\Omega_{2}}+K_{b s t s}^{\Omega_{2}} \cdot U_{b s}^{\Omega_{2}}+K_{b s t p_{3}}^{\Omega_{2}} \cdot U_{t p_{3}}^{\Omega_{2}}+K_{b s b p_{3}}^{\Omega_{2}} \cdot U_{b p_{3}}^{\Omega_{2}} \\
& P_{b p_{3}}^{\Omega_{2}}=K_{b p_{3} t s}^{\Omega_{2}} \cdot U_{t s}^{\Omega_{2}}+K_{b p_{3} t s}^{\Omega_{2}} \cdot U_{b s}^{\Omega_{2}}+K_{b p_{3} p_{3}}^{\Omega_{2}} \cdot U_{t p_{3}}^{\Omega_{2}}+K_{b p_{3} b p_{3}}^{\Omega_{2}} \cdot U_{b p_{3}}^{\Omega_{2}}
\end{aligned}
$$

Sabendo-se que:

$U_{t s}^{\Omega_{1}}=U_{b s}^{\Omega_{2}}$

$U_{t p_{3}}^{\Omega_{1}}=U_{b p_{3}}^{\Omega_{2}}$

$P_{t s}^{\Omega_{1}}=-P_{b s}^{\Omega_{2}}$

$P_{t p_{3}}^{\Omega_{1}}=-P_{b p_{3}}^{\Omega_{2}}$

Substituindo-se a equação (3.61), considerando-se as relações (3.65.1) e (3.65.2) nas relações (3.63) e (3.64), chega-se a: 


$$
\left[\begin{array}{cc}
-\left(K_{t s t s}^{\Omega_{1}}+K_{b s b s}^{\Omega_{2}}\right) & -\left(K_{t s t p_{3}}^{\Omega_{1}}+K_{b s b p_{3}}^{\Omega_{2}}\right) \\
-\left(K_{t p_{3} t s}^{\Omega_{1}}+K_{b p_{3} b s}^{\Omega_{2}}\right) & -\left(K_{t p_{3} t p_{3}}^{\Omega_{1}}+K_{b p_{3} b p_{3}}^{\Omega_{2}}\right)
\end{array}\right] \cdot\left\{\begin{array}{l}
U_{b s}^{\Omega_{2}} \\
U_{b p_{3}}^{\Omega_{2}}
\end{array}\right\}=\left[\begin{array}{cc}
K_{b s t s}^{\Omega_{2}} & K_{b s t p_{3}}^{\Omega_{2}} \\
K_{b p_{3} t s}^{\Omega_{2}} & K_{b p_{3} t p_{3}}^{\Omega_{2}}
\end{array}\right] \cdot\left\{\begin{array}{c}
U_{t s}^{\Omega_{2}} \\
U_{t p_{3}}^{\Omega_{2}}
\end{array}\right\}
$$

Ou reescrevendo-se a equação (3.66):

$$
\left\{\begin{array}{c}
U_{b s}^{\Omega_{2}} \\
U_{b p_{3}}^{\Omega_{2}}
\end{array}\right\}=\left[\begin{array}{ll}
\hat{K}_{b s t s}^{\Omega_{2}} & \hat{K}_{b s t p_{3}}^{\Omega_{2}} \\
\hat{K}_{b p_{3} t s}^{\Omega_{2}} & \hat{K}_{b p_{3} t p_{3}}^{\Omega_{2}}
\end{array}\right] \cdot\left\{\begin{array}{c}
U_{t s}^{\Omega_{2}} \\
U_{t p_{3}}^{\Omega_{2}}
\end{array}\right\}
$$

Aplicando-se a relação (3.67) na primeira e na terceira relação de (3.62) tem-se:

$$
\left[\begin{array}{cc}
\left(K_{t s t s}^{\Omega_{2}}+K_{t b s}^{\Omega_{2}} \cdot \hat{K}_{b s t s}^{\Omega_{2}}+K_{t s b_{3}}^{\Omega_{2}} \cdot \hat{K}_{b p_{3} t s}^{\Omega_{2}}\right) & \left(K_{t s t p_{3}}^{\Omega_{2}}+K_{t s b s}^{\Omega_{2}} \cdot \hat{K}_{b s t p_{3}}^{\Omega_{2}}+K_{t s p_{3}}^{\Omega_{2}} \cdot \hat{K}_{b p_{3} p_{3}}^{\Omega_{2}}\right) \\
\left(K_{t p_{3} t s}^{\Omega_{2}}+K_{t p_{3} b s}^{\Omega_{2} b} \cdot \hat{K}_{b s t s}^{\Omega_{2}}+K_{t p_{3} b p_{3}}^{\Omega_{2}} \cdot \hat{K}_{b p_{3} t s}^{\Omega_{2}}\right) & \left(K_{t p_{3} t p_{3}}^{\Omega_{2}}+K_{t p_{3} b s}^{\Omega_{2}} \cdot \hat{K}_{b s p_{3}}^{\Omega_{2}}+K_{t p_{3} b p_{3}}^{\Omega_{2}} \cdot \hat{K}_{b p_{3} t p_{3}}^{\Omega_{2}}\right)
\end{array}\right] \cdot\left\{\begin{array}{c}
U_{t s}^{\Omega_{2}} \\
U_{t p_{3}}^{\Omega_{2}}
\end{array}\right\}=\left\{\begin{array}{c}
P_{t s}^{\Omega_{2}} \\
P_{t p_{3}}^{\Omega_{2}}
\end{array}\right\}
$$

Ou

$$
\left\{\begin{array}{l}
P_{t s}^{\Omega_{2}} \\
P_{t p_{3}}^{\Omega_{2}}
\end{array}\right\}=\left[\begin{array}{cc}
\widetilde{K}_{t s t s}^{\Omega_{2}} & \widetilde{K}_{t s t p_{3}}^{\Omega_{2}} \\
\widetilde{K}_{t p_{3} t s}^{\Omega_{2}} & \widetilde{K}_{t p_{3} t p_{3}}^{\Omega_{2}}
\end{array}\right] \cdot\left\{\begin{array}{c}
U_{t s}^{\Omega_{2}} \\
U_{t p_{3}}^{\Omega_{2}}
\end{array}\right\}
$$

A equação (3.69) pode ser expandida já se considerando as posições da estaca 2 e 3 da terceira camada:

$$
\left[\begin{array}{cccc}
\widetilde{K}_{t s t s}^{\Omega_{2}} & \widetilde{K}_{t s t p_{1}}^{\Omega_{2}} & \widetilde{K}_{t s t p_{2}}^{\Omega_{2}} & \widetilde{K}_{t s t p_{3}}^{\Omega_{2}} \\
\widetilde{K}_{t p_{2} t s}^{\Omega_{2}} & \widetilde{K}_{t p_{1}}^{\Omega_{2}} & \widetilde{K}_{t p_{1}}^{\Omega_{2}} & \widetilde{K}_{t p_{2}}^{\Omega_{1}} \\
\widetilde{K}_{t p_{2}}^{\Omega_{2}} & \widetilde{K}_{t p_{2}}^{\Omega_{2} t p_{1}} & \widetilde{K}_{t p_{2} t p_{2}}^{\Omega_{2}} & \widetilde{K}_{t p_{2} t p_{3}}^{\Omega_{2}} \\
\widetilde{K}_{t p_{3} t s}^{\Omega_{2}} & \widetilde{K}_{t p_{3} t p_{1}}^{\Omega_{2}} & \widetilde{K}_{t p_{3} t p_{2}}^{\Omega_{2}} & \widetilde{K}_{t p_{3} t p_{3}}^{\Omega_{2}}
\end{array}\right] \cdot\left\{\begin{array}{c}
U_{t s}^{\Omega_{2}} \\
U_{t p_{1}}^{\Omega_{2}} \\
U_{t p_{2}}^{\Omega_{2}} \\
U_{t p_{3}}^{\Omega_{2}}
\end{array}\right\}=\left\{\begin{array}{c}
P_{t s}^{\Omega_{2}} \\
P_{t p_{1}}^{\Omega_{2}} \\
P_{t p_{2}}^{\Omega_{2}} \\
P_{t p_{3}}^{\Omega_{2}}
\end{array}\right\}
$$

\section{$3^{a}$ camada:}

A equação obtida para esta terceira camada fica: 


\begin{tabular}{|c|c|c|c|c|c|c|c|c|c|}
\hline$K_{t s t s}^{\Omega_{3}}$ & $K_{t s b s}^{\Omega_{3}}$ & $K_{t s t p_{1}}^{\Omega_{3}}$ & $K_{t s b p_{1}}^{\Omega_{3}}$ & $K_{t s t p_{2}}^{\Omega_{3}}$ & $K_{t s b p_{2}}^{\Omega_{3}}$ & $K_{t s t p_{3}}^{\Omega_{3}}$ & $K_{t s b p_{3}}^{\Omega_{3}}$ & $\left(U_{t s}^{\Omega_{3}}\right.$ & $\left(P_{t s}^{\Omega_{3}}\right.$ \\
\hline$K_{b s t s}^{\Omega_{3}}$ & $K_{b s b s}^{\Omega_{3}}$ & $K_{b s t p_{1}}^{\Omega_{3}}$ & $K_{b s b p_{1}}^{\Omega_{3}}$ & $K_{b s t p_{2}}^{\Omega_{3}}$ & $K_{b s b p_{2}}^{\Omega_{3}}$ & $K_{b s t p_{3}}^{\Omega_{3}}$ & $K_{b s b p_{3}}^{\Omega_{3}}$ & $U_{b s}^{\Omega_{3}}$ & $P_{b s}^{\Omega_{3}}$ \\
\hline$K_{t p_{1} t s}^{\Omega_{3}}$ & $K_{t p_{1} b s}^{\Omega_{3}}$ & $K_{t p_{1} p_{1}}^{\Omega_{3}}$ & $K_{t p_{1} b p_{1}}^{\Omega_{3}}$ & $K_{t p_{1} t p_{2}}^{\Omega_{3}}$ & $K_{t p_{1} b p_{2}}^{\Omega_{3}}$ & $K_{t p_{1} t p_{3}}^{\Omega_{3}}$ & $K_{t p_{1} t p_{3}}^{\Omega_{3}}$ & $U_{t p_{1}}^{\Omega_{3}}$ & $P_{t p_{1}}^{\Omega_{3}}$ \\
\hline$K_{b p_{1} t s}^{\Omega_{3}}$ & $K_{b p_{1} b s}^{\Omega_{3}}$ & $K_{b p_{1} p_{1}}^{\Omega_{3}}$ & $K_{b p_{1} b p_{1}}^{\Omega_{3}}$ & $K_{b p_{1} t p_{2}}^{\Omega_{3}}$ & $K_{b p_{1} b p_{2}}^{\Omega_{3}}$ & $K_{b p_{1} t p_{3}}^{\Omega_{3}}$ & $K_{b p_{1} t p_{3}}^{\Omega_{3}}$ & $U_{b p_{1}}^{\Omega_{3}}$ & $P_{b p_{1}}^{\Omega_{3}}$ \\
\hline$K_{t p_{2} t s}^{\Omega_{3}}$ & $K_{t p_{2} b s}^{\Omega_{3}}$ & $K_{t p_{2} t p_{1}}^{\Omega_{3}}$ & $K_{t p_{2} b p_{1}}^{\Omega_{3}}$ & $K_{t p_{2} b p_{1}}^{\Omega_{3}}$ & $K_{t p_{2} b p_{2}}^{\Omega_{3}}$ & $K_{t p_{2} t p_{3}}^{\Omega_{3}}$ & $K_{t p_{2} t p_{3}}^{\Omega_{3}}$ & $U_{t p_{2}}^{\Omega_{3}}$ & $P_{t p_{2}}^{\Omega_{3}}$ \\
\hline$K_{b p_{2} t s}^{\Omega_{3}}$ & $K_{b p_{2} b s}^{\Omega_{3}}$ & $K_{b p_{2} t p_{1}}^{\Omega_{3}}$ & $K_{b p_{2} b p_{1}}^{\Omega_{3}}$ & $K_{b p_{2} t p_{2}}^{\Omega_{3}}$ & $K_{b p_{2} b p_{2}}^{\Omega_{3}}$ & $K_{b p_{2} t p_{3}}^{\Omega_{3}}$ & $K_{b p_{2} t p_{3}}^{\Omega_{3}}$ & $U_{b p_{2}}^{\Omega_{3}}$ & $P_{b p_{2}}^{\Omega_{3}}$ \\
\hline$K_{t p_{3} t s}^{\Omega_{3}}$ & $K_{t_{p} b s}^{\Omega_{3}}$ & $K_{t p 3 t p_{1}}^{\Omega_{3}}$ & $K_{t p_{3} b p_{1}}^{\Omega_{3}}$ & $K_{t p_{3} b p_{1}}^{\Omega_{3}}$ & $K_{t p_{3} b p_{2}}^{\Omega_{3}}$ & $K_{t p_{3} t p_{3}}^{\Omega_{3}}$ & $K_{t p_{3} t p_{3}}^{\Omega_{3}}$ & $U_{t p_{3}}^{\Omega_{3}}$ & $P_{t p_{3}}^{\Omega_{3}}$ \\
\hline$K_{b p_{3} t s}^{\Omega_{3}}$ & $K_{b p_{3} b s}^{\Omega_{3}}$ & $K_{b p_{3} t p_{1}}^{\Omega_{3}}$ & $K_{b p_{3} b p_{1}}^{\Omega_{3}}$ & $K_{b p_{3} t p_{2}}^{\Omega_{3}}$ & $K_{b p_{3} b p_{2}}^{\Omega_{3}}$ & $K_{b p_{3} t p_{3}}^{\Omega_{3}}$ & $K_{b p_{3} t p_{3}}^{\Omega_{3} p_{3}}$ & $U_{b p_{3}}^{\Omega_{3}}$ & $P_{b p_{3}}^{\Omega_{3}}$ \\
\hline
\end{tabular}

Escrevendo-se a segunda, a quarta, a sexta e a oitava expressão de (3.71), tem-se:

$$
\begin{aligned}
& P_{b s}^{\Omega_{3}}=K_{b s s t s}^{\Omega_{3}} \cdot U_{t s}^{\Omega_{3}}+K_{b s t s}^{\Omega_{3}} \cdot U_{b s}^{\Omega_{3}}+K_{b s t p_{1}}^{\Omega_{3}} \cdot U_{t p_{1}}^{\Omega_{3}}+K_{b s b p_{1}}^{\Omega_{3}} \cdot U_{b p_{1}}^{\Omega_{3}}+K_{b s t p_{2}}^{\Omega_{3}} \cdot U_{t p_{2}}^{\Omega_{3}}+ \\
& K_{b s b p_{2}}^{\Omega_{3}} \cdot U_{b p_{2}}^{\Omega_{3}}+K_{b s t p_{3}}^{\Omega_{3}} \cdot U_{t p_{3}}^{\Omega_{3}}+K_{b s b p_{3}}^{\Omega_{3}} \cdot U_{b p_{3}}^{\Omega_{3}} \\
& P_{b p}^{\Omega_{3}}=K_{b p_{1} t s}^{\Omega_{3}} \cdot U_{t s}^{\Omega_{3}}+K_{b p_{1} / s}^{\Omega_{3}} \cdot U_{b s}^{\Omega_{3}}+K_{b p_{1} t_{1}}^{\Omega_{3}} \cdot U_{t p_{1}}^{\Omega_{3}}+K_{b p_{1} b_{1}}^{\Omega_{3}} \cdot U_{b p_{1}}^{\Omega_{3}}+K_{b p_{1} p_{2}}^{\Omega_{3}} \cdot U_{t p_{2}}^{\Omega_{3}}+ \\
& K_{b p_{1} b p_{2}}^{\Omega_{3}} \cdot U_{b p_{2}}^{\Omega_{3}}+K_{b p_{1}}^{\Omega_{3} p_{3}} \cdot U_{t p_{3}}^{\Omega_{3}}+K_{b p_{1} p_{3}}^{\Omega_{3}} \cdot U_{b p_{3}}^{\Omega_{3}} \\
& P_{b p_{2}}^{\Omega_{3}}=K_{b p_{2} t s}^{\Omega_{3}} \cdot U_{t s}^{\Omega_{3}}+K_{b p_{2} t s}^{\Omega_{3}} \cdot U_{b s}^{\Omega_{3}}+K_{b p_{2} p_{1}}^{\Omega_{3}} \cdot U_{t p_{1}}^{\Omega_{3}}+K_{b p_{2} b p_{1}}^{\Omega_{3}} \cdot U_{b p_{1}}^{\Omega_{3}}+K_{b p_{2} p_{2}}^{\Omega_{3}} \cdot U_{t p_{2}}^{\Omega_{3}}+ \\
& K_{b p_{2} b p_{2}}^{\Omega_{3}} \cdot U_{b p_{2}}^{\Omega_{3}}+K_{b p_{2} p_{3}}^{\Omega_{3}} \cdot U_{t p_{3}}^{\Omega_{3}}+K_{b p_{2} b p_{3}}^{\Omega_{3}} \cdot U_{b p_{3}}^{\Omega_{3}} \\
& P_{b p_{3}}^{\Omega_{3}}=K_{b p_{3} t s}^{\Omega_{3}} \cdot U_{t s}^{\Omega_{3}}+K_{b p_{3} t s}^{\Omega_{3}} \cdot U_{b s}^{\Omega_{3}}+K_{b p_{3} p_{1}}^{\Omega_{3}} \cdot U_{t p_{1}}^{\Omega_{3}}+K_{b p_{3} b p_{1}}^{\Omega_{3}} \cdot U_{b p_{1}}^{\Omega_{3}}+K_{b p_{3} p_{2}}^{\Omega_{3}} \cdot U_{t p_{2}}^{\Omega_{3}}+K_{b p_{3} b p_{2}}^{\Omega_{3}} \cdot U_{b p_{2}}^{\Omega_{3}}+ \\
& K_{b p_{3} p_{3}}^{\Omega_{3}} \cdot U_{t p_{3}}^{\Omega_{3}}+K_{b p_{3} b p_{3}}^{\Omega_{3}} \cdot U_{b p_{3}}^{\Omega_{3}}
\end{aligned}
$$

\section{Sabendo-se que:}

$U_{t s}^{\Omega_{2}}=U_{b s}^{\Omega_{3}}$

$U_{t p_{1}}^{\Omega_{2}}=U_{b p_{1}}^{\Omega_{3}}$

$U_{t p_{2}}^{\Omega_{2}}=U_{b p_{2}}^{\Omega_{3}}$

$U_{t p_{3}}^{\Omega_{2}}=U_{b p_{3}}^{\Omega_{3}}$ 
$P_{t s}^{\Omega_{2}}=-P_{b s}^{\Omega_{3}}$

$P_{t p_{1}}^{\Omega_{2}}=-P_{b p_{1}}^{\Omega_{3}}$

$P_{t p_{2}}^{\Omega_{2}}=-P_{b p_{2}}^{\Omega_{3}}$

$P_{t p_{3}}^{\Omega_{2}}=-P_{b p_{3}}^{\Omega_{3}}$

Substituindo-se a equação (3.70), considerando-se as relações (3.76.1) e (3.76.2), nas relações (3.72) a (3.75), chega-se a:

$$
\begin{aligned}
& {\left[\begin{array}{cccc}
-\left(\widetilde{K}_{t s t s}^{\Omega_{2}}+K_{b s b s}^{\Omega_{3}}\right) & -\left(\widetilde{K}_{t s t p_{1}}^{\Omega_{2}}+K_{b s p_{1}}^{\Omega_{3}}\right) & -\left(\widetilde{K}_{t s t p_{2}}^{\Omega_{2}}+K_{b s b p_{2}}^{\Omega_{3}}\right) & -\left(\widetilde{K}_{t s t p_{3}}^{\Omega_{2}}+K_{b s b p_{3}}^{\Omega_{3}}\right) \\
-\left(\widetilde{K}_{t p_{1} t s}^{\Omega_{2}}+K_{b p_{1} b s}^{\Omega_{3}}\right) & -\left(\widetilde{K}_{t p_{2} t p_{1}}^{\Omega_{2}}+K_{b p_{1} b p_{1}}^{\Omega_{1}}\right) & -\left(\widetilde{K}_{t p_{1} t p_{2}}^{\Omega_{2}}+K_{b p_{1} b p_{2}}^{\Omega_{2}}\right) & -\left(\widetilde{K}_{t p_{2} t p_{3}}^{\Omega_{2}}+K_{b p_{1} b p_{3}}^{\Omega_{3}}\right) \\
-\left(\widetilde{K}_{t p_{2} t s}^{\Omega_{2}}+K_{b p_{2} b s}^{\Omega_{3}}\right) & -\left(\widetilde{K}_{t p_{2} t p_{1}}^{\Omega_{2}}+K_{b p_{2} b p_{1}}^{\Omega_{1}}\right) & -\left(\widetilde{K}_{t p_{2} t p_{2}}^{\Omega_{2}}+K_{b p_{2} b p_{2}}^{\Omega_{3}}\right) & -\left(\widetilde{K}_{t p_{2} t p_{3}}^{\Omega_{2}}+K_{b p_{2} b p_{3}}^{\Omega_{3}}\right) \\
-\left(\widetilde{K}_{t p_{3} t s}^{\Omega_{2}}+K_{b p_{3} b s}^{\Omega_{3}}\right) & -\left(\widetilde{K}_{t p_{3} t p_{1}}^{\Omega_{2}}+K_{b p_{3} b p_{1}}^{\Omega_{3}}\right) & -\left(\widetilde{K}_{t p_{3} t p_{2}}^{\Omega_{2}}+K_{b p_{3} b p_{2}}^{\Omega_{3}}\right) & -\left(\widetilde{K}_{t p_{3} t p_{3}}^{\Omega_{2}}+K_{b p_{3} b p_{3}}^{\Omega_{3}}\right)
\end{array}\right] \cdot\left\{\begin{array}{c}
U_{b s}^{\Omega_{3}} \\
U_{b p_{1}}^{\Omega_{3}} \\
U_{b p_{2}}^{\Omega_{3}} \\
U_{b p_{3}}^{\Omega_{3}}
\end{array}\right\}} \\
& {\left[\begin{array}{llll}
K_{b s t s}^{\Omega_{3}} & K_{b s t p_{1}}^{\Omega_{3}} & K_{b s t p_{2}}^{\Omega_{3}} & K_{b s t p_{3}}^{\Omega_{3}} \\
K_{b p_{1} t s}^{\Omega_{3}} & K_{b p_{1} t p_{1}}^{\Omega_{1}} & K_{b p_{1} t p_{2}}^{\Omega_{3}} & K_{b p_{1} t p_{3}}^{\Omega_{3}} \\
K_{b p_{2} t s}^{\Omega_{3}} & K_{b p_{2} t p_{1}}^{\Omega_{3}} & K_{b p_{2} t p_{2}}^{\Omega_{3}} & K_{b p_{2} t p_{3}}^{\Omega_{3}} \\
K_{b p_{3} t s}^{\Omega_{3}} & K_{b p_{3} t p_{1}}^{\Omega_{3}} & K_{b p_{3} t p_{2}}^{\Omega_{3}} & K_{b p_{3} t p_{3}}^{\Omega_{3}}
\end{array}\right] \cdot\left\{\begin{array}{c}
U_{t s}^{\Omega_{3}} \\
U_{t p_{1}}^{\Omega_{3}} \\
U_{t p_{2}}^{\Omega_{3}} \\
U_{t p_{3}}^{\Omega_{3}}
\end{array}\right\}}
\end{aligned}
$$

Ou transformando-se a expressão (3.77):

$$
\left[\begin{array}{cccc}
\hat{K}_{t s t s}^{\Omega_{3}} & \hat{K}_{t s t p_{1}}^{\Omega_{3}} & \hat{K}_{t s t p_{2}}^{\Omega_{3}} & \hat{K}_{t s t p_{3}}^{\Omega_{3}} \\
\hat{K}_{t p_{3} t s}^{\Omega_{3}} & \hat{K}_{t p_{3} t p_{1}}^{\Omega_{2}} & \hat{K}_{t p_{1}}^{\Omega_{3}} & \hat{K}_{t p_{3} t p_{3}}^{\Omega_{3}} \\
\hat{K}_{t p_{3} t s}^{\Omega_{3}} & \hat{K}_{t p_{3}}^{\Omega_{2}} & \hat{K}_{t p_{3}}^{\Omega_{3} t p_{2}} & \hat{K}_{t p_{2} t p_{3}}^{\Omega_{3}} \\
\hat{K}_{t p_{3} t s}^{\Omega_{3}} & \hat{K}_{t p_{3} t p_{1}}^{\Omega_{3}} & \hat{K}_{t p_{3} t p_{2}}^{\Omega_{3}} & \hat{K}_{t p_{3} t p_{3}}^{\Omega_{3}}
\end{array}\right] \cdot\left\{\begin{array}{c}
U_{t s}^{\Omega_{3}} \\
U_{t p_{1}}^{\Omega_{3}} \\
U_{t p_{3}}^{\Omega_{3}} \\
U_{t p_{3}}^{\Omega_{3}}
\end{array}\right\}=\left\{\begin{array}{c}
U_{b s}^{\Omega_{3}} \\
U_{b p_{1}}^{\Omega_{3}} \\
U_{b p_{2}}^{\Omega_{3}} \\
U_{b p_{3}}^{\Omega_{3}}
\end{array}\right\}
$$

Aplicando-se a relação (3.78) na primeira, terceira, na quinta e na sétima relação de (3.71), tem-se: 


$$
\left\{\begin{array}{c}
P_{t s}^{\Omega_{3}} \\
P_{t p_{1}}^{\Omega_{3}} \\
P_{t p_{2}}^{\Omega_{3}} \\
P_{t p_{3}}^{\Omega_{3}}
\end{array}\right\}=\left[\begin{array}{cccc}
\widetilde{K}_{t s t s}^{\Omega_{3}} & \widetilde{K}_{t s t p_{1}}^{\Omega_{3}} & \widetilde{K}_{t s t p_{2}}^{\Omega_{3}} & \widetilde{K}_{t s t p_{3}}^{\Omega_{3}} \\
\widetilde{K}_{t p_{3} t s}^{\Omega_{3}} & \widetilde{K}_{t p_{3} t p_{1}}^{\Omega_{3}} & \widetilde{K}_{t p_{3} t p_{2}}^{\Omega_{3}} & \widetilde{K}_{t p_{3} t p_{3}}^{\Omega_{3}} \\
\widetilde{K}_{t p_{3} t s}^{\Omega_{2}} & \widetilde{K}_{t p_{2} t p_{1}}^{\Omega_{3}} & \widetilde{K}_{t p_{2} t p_{2}}^{\Omega_{3}} & \widetilde{K}_{t p_{3} t p_{3}}^{\Omega_{3}} \\
\widetilde{K}_{t p_{3} t s}^{\Omega_{3}} & \widetilde{K}_{t p_{3} t p_{1}}^{\Omega_{3}} & \widetilde{K}_{t p_{3} t p_{2}}^{\Omega_{3}} & \widetilde{K}_{t p_{3} t p_{3}}^{\Omega_{3}}
\end{array}\right] \cdot\left\{\begin{array}{c}
U_{t s}^{\Omega_{3}} \\
U_{t p_{3}}^{\Omega_{3}} \\
U_{t p_{3}}^{\Omega_{3}} \\
U_{t p_{3}}^{\Omega_{3}}
\end{array}\right\}
$$

$\widetilde{K}_{t s t s}=K_{t s t s}^{\Omega_{3}}+K_{t s b s}^{\Omega_{3}} \cdot \hat{K}_{t s t s}^{\Omega_{3}}+K_{t s b p_{1}}^{\Omega_{3}} \cdot \hat{K}_{t p_{1} t s}^{\Omega_{3}}+K_{t s b p_{2}}^{\Omega_{3}} \cdot \hat{K}_{t p_{2} t s}^{\Omega_{3}}+K_{t s b p_{3}}^{\Omega_{3}} \cdot \hat{K}_{t p_{3} t s}^{\Omega_{3}}$

$\widetilde{K}_{t s t p_{1}}=K_{t s t p_{1}}^{\Omega_{3}}+K_{t s b s}^{\Omega_{3}} \cdot \hat{K}_{t s t p_{1}}^{\Omega_{3}}+K_{t s b p_{1}}^{\Omega_{3}} \cdot \hat{K}_{t p_{1} t p_{1}}^{\Omega_{3}}+K_{t s b p_{2}}^{\Omega_{3}} \cdot \hat{K}_{t p_{2} t p_{1}}^{\Omega_{3}}+K_{t s b p_{3}}^{\Omega_{3}} \cdot \hat{K}_{t p_{3} t p_{1}}^{\Omega_{3}}$

$\widetilde{K}_{t s t p_{2}}=K_{t s t p_{2}}^{\Omega_{3}}+K_{t s b s}^{\Omega_{3}} \cdot \hat{K}_{t s t p_{2}}^{\Omega_{3}}+K_{t s b p_{1}}^{\Omega_{3}} \cdot \hat{K}_{t p_{1} p_{2}}^{\Omega_{3}}+K_{t s b p_{2}}^{\Omega_{3}} \cdot \hat{K}_{t p_{2} t p_{2}}^{\Omega_{3}}+K_{t s b p_{3}}^{\Omega_{3}} \cdot \hat{K}_{t p_{3} t p_{2}}^{\Omega_{3}}$

$\widetilde{K}_{t s t p_{3}}=K_{t s t p_{3}}^{\Omega_{3}}+K_{t s b s}^{\Omega_{3}} \cdot \hat{K}_{t s t p_{3}}^{\Omega_{3}}+K_{t s b p_{1}}^{\Omega_{3}} \cdot \hat{K}_{t p_{1} p_{3}}^{\Omega_{3}}+K_{t s b p_{2}}^{\Omega_{3}} \cdot \hat{K}_{t p_{2} t p_{3}}^{\Omega_{3}}+K_{t s b p_{3}}^{\Omega_{3}} \cdot \hat{K}_{t p_{3} t p_{3}}^{\Omega_{3}}$

$\tilde{K}_{t p_{1} t s}=K_{t p_{1} t s}^{\Omega_{3}}+K_{t p_{1} b s}^{\Omega_{3}} \cdot \hat{K_{t s t s}}+K_{t p_{1} b p_{1}}^{\Omega_{3}} \cdot \hat{K}_{t p_{1} t s}^{\Omega_{3}}+K_{t p_{1} b p_{2}}^{\Omega_{3}} \cdot \hat{K_{t p_{2} t s}}+K_{t p_{1} b p_{3}}^{\Omega_{3}} \cdot \hat{K}_{t p_{3} t s}$

$\tilde{K}_{t p_{1} t p_{1}}=K_{t p_{1} t p_{1}}^{\Omega_{3}}+K_{t p_{1} b s}^{\Omega_{3}} \cdot \hat{K}_{t s t p_{1}}^{\Omega_{3}}+K_{t p_{1} b p_{1}}^{\Omega_{3}} \cdot \hat{K}_{t p_{1} t p_{1}}^{\Omega_{3}}+K_{t p_{1} b p_{2}}^{\Omega_{3}} \cdot \hat{K_{t p_{2} t p_{1}}}+K_{t p_{1} b p_{3}}^{\Omega_{3}} \cdot \hat{K_{t p_{3}} t p_{1}}$

$\tilde{K}_{t p_{1} t p_{2}}=K_{t p_{1} t p_{2}}^{\Omega_{3}}+K_{t p_{1} b s}^{\Omega_{3}} \cdot \hat{K}_{t s t p_{2}}^{\Omega_{3}}+K_{t p_{1} b p_{1}}^{\Omega_{3}} \cdot \hat{K}_{t p_{1} t p_{2}}^{\Omega_{3}}+K_{t p_{1} b p_{2}}^{\Omega_{3}} \cdot \hat{K_{t p_{2}} t p_{2}}+K_{t p_{1} b p_{3}}^{\Omega_{3}} \cdot \hat{K}_{t p_{3} t p_{2}}^{\Omega_{3}}$

$\tilde{K}_{t p_{1} t p_{3}}=K_{t p_{1} t p_{3}}^{\Omega_{3}}+K_{t p_{1} b s}^{\Omega_{3}} \cdot \hat{K}_{t s t p_{3}}^{\Omega_{3}}+K_{t p_{1} b p_{1}}^{\Omega_{3}} \cdot \hat{K}_{t p_{1} t p_{3}}^{\Omega_{3}}+K_{t p_{1} b p_{2}}^{\Omega_{3}} \cdot \hat{K}_{t p_{2} t p_{3}}+K_{t p_{1} b p_{3}}^{\Omega_{3}} \cdot \hat{K}_{t p_{3} t p_{3}}$

$\widetilde{K}_{t p_{2} t s}=K_{t p_{2} t s}^{\Omega_{3}}+K_{t p_{2} b s}^{\Omega_{3}} \cdot \hat{K}_{t s t s}^{\Omega_{3}}+K_{t p_{2} b p_{1}}^{\Omega_{3}} \cdot \hat{K}_{t p_{1} t s}^{\Omega_{3}}+K_{t p_{2} b p_{2}}^{\Omega_{3}} \cdot \hat{K}_{t p_{2} t s}^{\Omega_{3}}+K_{t p_{1} b p_{3}}^{\Omega_{3}} \cdot \hat{K}_{t p_{3} t s}^{\Omega_{3}}$

$\widetilde{K}_{t p_{2} t p_{1}}=K_{t p_{2} t p_{1}}^{\Omega_{3}}+K_{t p_{2} b s}^{\Omega_{3}} \cdot \hat{K}_{t s t p_{1}}^{\Omega_{3}}+K_{t p_{2} b p_{1}}^{\Omega_{3}} \cdot \hat{K}_{t p_{1} t p_{1}}^{\Omega_{3}}+K_{t p_{2} b p_{2}}^{\Omega_{3}} \cdot \hat{K}_{t p_{2} t p_{1}}^{\Omega_{3}}+K_{t p_{1} b p_{3}}^{\Omega_{3}} \cdot \hat{K}_{t p_{3} t p_{1}}^{\Omega_{3}}$

$\widetilde{K}_{t p_{2} t p_{2}}=K_{t p_{2} t p_{2}}^{\Omega_{3}}+K_{t p_{2} b s}^{\Omega_{3}} \cdot \hat{K}_{t s t p_{2}}^{\Omega_{3}}+K_{t p_{2} b p_{1}}^{\Omega_{3}} \cdot \hat{K}_{t p_{1} t p_{2}}^{\Omega_{3}}+K_{t p_{2} b p_{2}}^{\Omega_{3}} \cdot \hat{K}_{t p_{2} t p_{2}}^{\Omega_{3}}+K_{t p_{1} b p_{3}}^{\Omega_{3}} \cdot \hat{K}_{t p_{3} t p_{2}}^{\Omega_{3}}$

$\widetilde{K}_{t p_{2} t p_{3}}=K_{t p_{2} t p_{3}}^{\Omega_{3}}+K_{t p_{2} b s}^{\Omega_{3}} \cdot \hat{K}_{t s t p_{3}}^{\Omega_{3}}+K_{t p_{2} b p_{1}}^{\Omega_{3}} \cdot \hat{K}_{t p_{1} t p_{3}}^{\Omega_{3}}+K_{t p_{2} b p_{2}}^{\Omega_{3}} \cdot \hat{K}_{t p_{2} t p_{3}}^{\Omega_{3}}+K_{t p_{2} b p_{3}}^{\Omega_{3}} \cdot \hat{K}_{t p_{3} t p_{3}}^{\Omega_{3}}$

$\widetilde{K}_{t p_{3} t s}=K_{t p 3 t s}^{\Omega_{3}}+K_{t p_{2} b s}^{\Omega_{3}} \cdot \hat{K}_{t s t s}^{\Omega_{3}}+K_{t p_{3} b p_{1}}^{\Omega_{3}} \cdot \hat{K}_{t p_{1} t s}^{\Omega_{3}}+K_{t p_{33} b p_{2}}^{\Omega_{3}} \cdot \hat{K}_{t p_{2} t s}^{\Omega_{3}}+K_{t p_{1} b p_{3}}^{\Omega_{3}} \cdot \hat{K}_{t p_{3} t s}^{\Omega_{3}}$

$\widetilde{K}_{t p_{3} t p_{1}}=K_{t p_{3} t p_{1}}^{\Omega_{3}}+K_{t p_{2} b s}^{\Omega_{3}} \cdot \hat{K}_{t s t p_{1}}^{\Omega_{3}}+K_{t p_{3} b p_{1}}^{\Omega_{3}} \cdot \hat{K}_{t p_{1} t p_{1}}^{\Omega_{3}}+K_{t p_{3} b p_{2}}^{\Omega_{3}} \cdot \hat{K}_{t p_{2} t p_{1}}^{\Omega_{3}}+K_{t p_{1} b p_{3}}^{\Omega_{3}} \cdot \hat{K}_{t p_{3} t p_{1}}^{\Omega_{3}}$

$\widetilde{K}_{t p_{3} t p_{2}}=K_{t p_{3} t p_{2}}^{\Omega_{3}}+K_{t p_{2} b s}^{\Omega_{3}} \cdot \hat{K}_{t s t p_{2}}^{\Omega_{3}}+K_{t p_{3} b p_{1}}^{\Omega_{3}} \cdot \hat{K}_{t p_{1} t p_{2}}^{\Omega_{3}}+K_{t p_{2} b p_{2}}^{\Omega_{3}} \cdot \hat{K}_{t p_{2} t p_{2}}^{\Omega_{3}}+K_{t p_{1} b p_{3}}^{\Omega_{3}} \cdot \hat{K}_{t p_{3} t p_{2}}^{\Omega_{3}}$

$\widetilde{K}_{t p_{3} t p_{3}}=K_{t p_{3} t p_{3}}^{\Omega_{3}}+K_{t p_{2} b s}^{\Omega_{3}} \cdot \hat{K}_{t s t p_{3}}^{\Omega_{3}}+K_{t p_{3} b p_{1}}^{\Omega_{3}} \cdot \hat{K}_{t p_{1} t p_{3}}^{\Omega_{3}}+K_{t p_{2} b p_{2}}^{\Omega_{3}} \cdot \hat{K}_{t p_{2} t p_{3}}^{\Omega_{3}}+K_{t p_{2} b p_{3}}^{\Omega_{3}} \cdot \hat{K}_{t p_{3} t p_{3}}^{\Omega_{3}}$ 
$4^{a}$ camada:

A equação obtida para esta quarta camada fica:

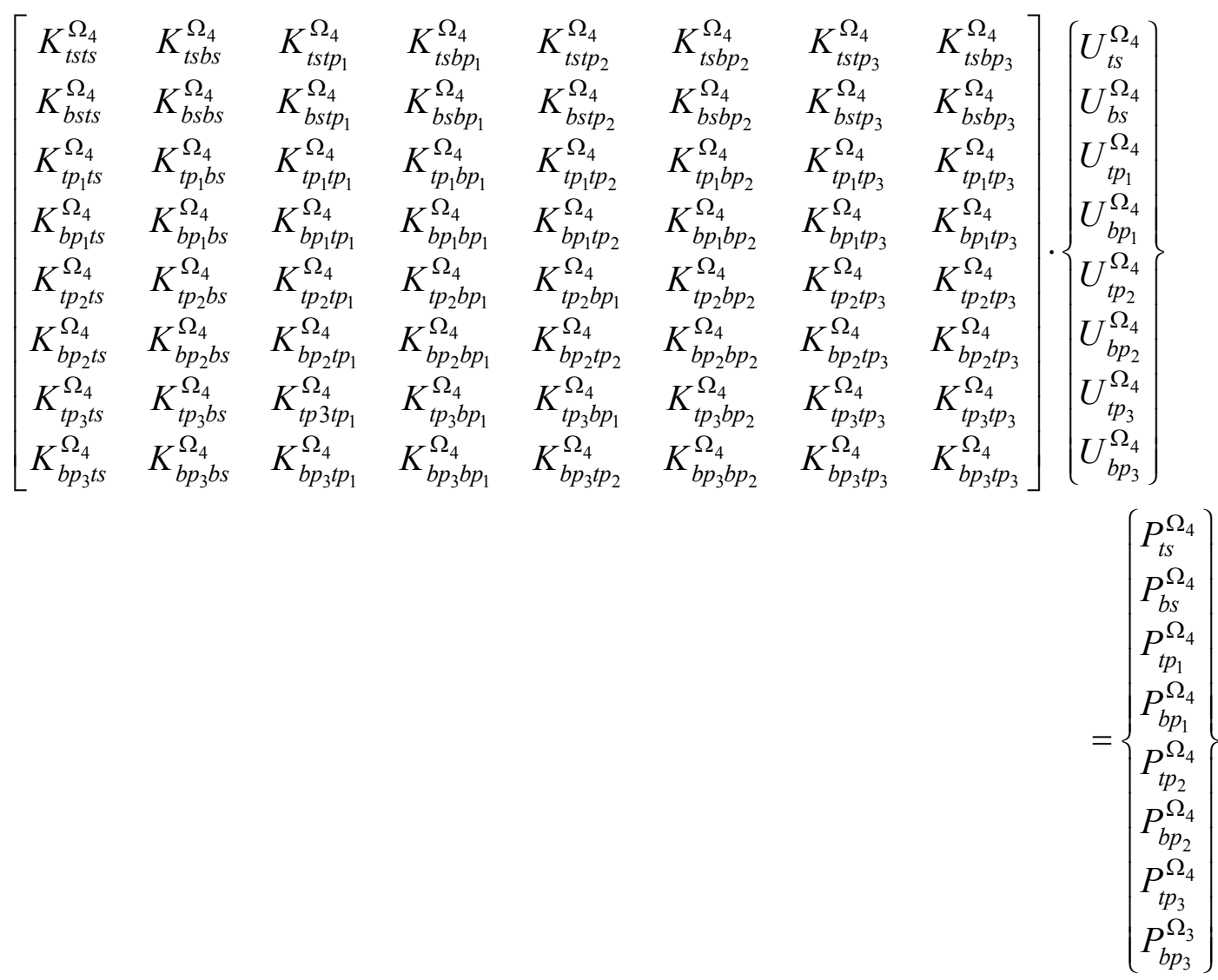

Da mesma forma como fora feito para a $3^{a}$ camada, escrevendo-se a segunda, a quarta, a sexta e a oitava expressão de (3.84), tem-se:

$$
\begin{aligned}
& P_{b s}^{\Omega_{4}}= K_{b s s s}^{\Omega_{4}} \cdot U_{t s}^{\Omega_{4}}+K_{b s t s}^{\Omega_{4}} \cdot U_{b s}^{\Omega_{4}}+K_{b s t p_{1}}^{\Omega_{4}} \cdot U_{t p_{1}}^{\Omega_{4}}+K_{b s b p_{1}}^{\Omega_{4}} \cdot U_{b p_{1}}^{\Omega_{4}}+K_{b s t p_{2}}^{\Omega_{4}} \cdot U_{t p_{2}}^{\Omega_{4}}+ \\
& K_{b s b p_{2}}^{\Omega_{4}} \cdot U_{b p_{2}}^{\Omega_{4}}+K_{b s t p_{3}}^{\Omega_{4}} \cdot U_{t p_{3}}^{\Omega_{4}}+K_{b s b p_{3}}^{\Omega_{4}} \cdot U_{b p_{3}}^{\Omega_{4}} \\
& P_{b p_{1}}^{\Omega 4}=K_{b p_{1} t s}^{\Omega_{4}} \cdot U_{t s}^{\Omega_{4}}+K_{b p_{1} t s}^{\Omega_{4}} \cdot U_{b s}^{\Omega_{4}}+K_{b p_{1} p_{1}}^{\Omega_{4}} \cdot U_{t p_{1}}^{\Omega_{4}}+K_{b p_{1} b p_{1}}^{\Omega_{4}} \cdot U_{b p_{1}}^{\Omega_{4}}+K_{b p_{1} t p_{2}}^{\Omega_{4}} \cdot U_{t p_{2}}^{\Omega_{4}}+ \\
& K_{b p_{1} b p_{2}}^{\Omega_{4}} \cdot U_{b p_{2}}^{\Omega_{4}}+K_{b p_{1} t p_{3}}^{\Omega_{4}} \cdot U_{t p_{3}}^{\Omega_{4}}+K_{b p_{1} b p_{3}}^{\Omega_{4}} \cdot U_{b p_{3}}^{\Omega_{4}} \\
& P_{b p_{2}}^{\Omega_{4}}=K_{b p_{2} t s}^{\Omega_{4}} \cdot U_{t s}^{\Omega_{4}}+K_{b p_{2} t s}^{\Omega_{4}} \cdot U_{b s}^{\Omega_{4}}+K_{b p_{2} p_{1}}^{\Omega_{4}} \cdot U_{t p_{1}}^{\Omega_{4}}+K_{b p_{2} p_{1}}^{\Omega_{4}} \cdot U_{b p_{1}}^{\Omega_{4}}+K_{b p_{2} t p_{2}}^{\Omega_{4}} \cdot U_{t p_{2}}^{\Omega_{4}}+ \\
& K_{b p_{2} b p_{2}}^{\Omega_{4}} \cdot U_{b p_{2}}^{\Omega_{4}}+K_{b p_{2} t p_{3}}^{\Omega_{4}} \cdot U_{t p_{3}}^{\Omega_{4}}+K_{b p_{2} b p_{3}}^{\Omega_{4}} \cdot U_{b p_{3}}^{\Omega_{4}} \\
& P_{b p_{3}}^{\Omega_{4}}=K_{b p_{3} t s}^{\Omega_{4}} \cdot U_{t s}^{\Omega_{4}}+K_{b p_{3} t s}^{\Omega_{4}} \cdot U_{b s}^{\Omega_{4}}+K_{b p_{3} p_{1}}^{\Omega_{4}} \cdot U_{t p_{1}}^{\Omega_{4}}+K_{b p_{3} b p_{1}}^{\Omega_{4}} \cdot U_{b p_{1}}^{\Omega_{4}}+ \\
& K_{b p_{3} t p_{2}}^{\Omega_{4}} \cdot U_{t p_{2}}^{\Omega_{4}}+K_{b p_{3} b p_{2}}^{\Omega_{4}} \cdot U_{b p_{2}}^{\Omega_{4}}+K_{b p_{3} t p_{3}}^{\Omega_{4}} \cdot U_{t p_{3}}^{\Omega_{4}}+K_{b p_{3} b p_{3}}^{\Omega_{4}} \cdot U_{b p_{3}}^{\Omega_{4}}
\end{aligned}
$$


Usando-se as condições de compatibilidade e equilíbrio entre as camadas adjacentes, ou seja:

$$
\begin{aligned}
& U_{t s}^{\Omega_{3}}=U_{b s}^{\Omega_{4}} \\
& U_{t p_{1}}^{\Omega_{3}}=U_{b p_{1}}^{\Omega_{4}} \\
& U_{t p_{2}}^{\Omega_{3}}=U_{b p_{2}}^{\Omega_{4}} \\
& U_{t p_{3}}^{\Omega_{3}}=U_{b p_{3}}^{\Omega_{4}} \\
& P_{t s}^{\Omega_{3}}=-P_{b s}^{\Omega_{4}} \\
& P_{t p_{1}}^{\Omega_{3}}=-P_{b p_{1}}^{\Omega_{4}} \\
& P_{t p_{2}}^{\Omega_{3}}=-P_{b p_{2}}^{\Omega_{4}} \\
& P_{t p_{3}}^{\Omega_{3}}=-P_{b p_{3}}^{\Omega_{4}}
\end{aligned}
$$

Substituindo-se a equação (3.79), considerando-se as relações (3.89.1) e (3.89.2) nas relações (3.85) a (3.88), chega-se a:

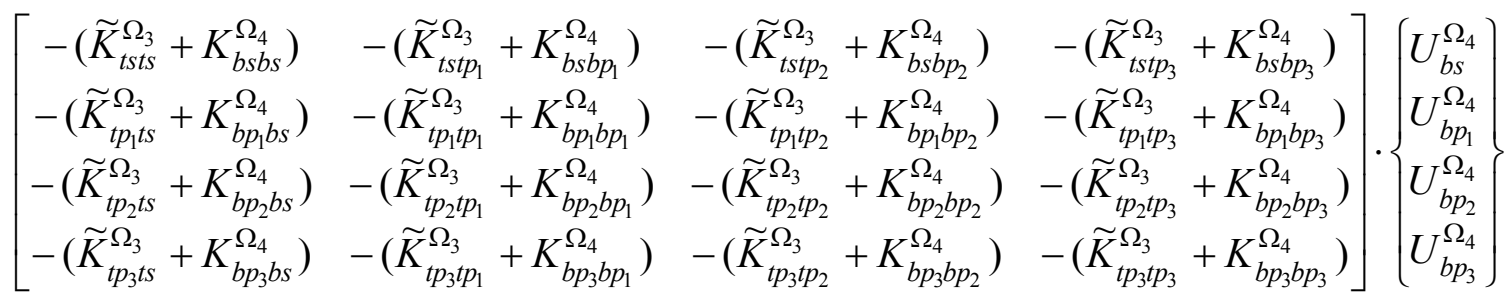

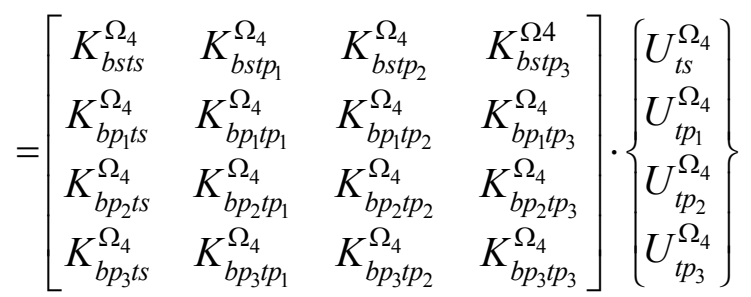

Ou transformando-se a expressão (3.90):

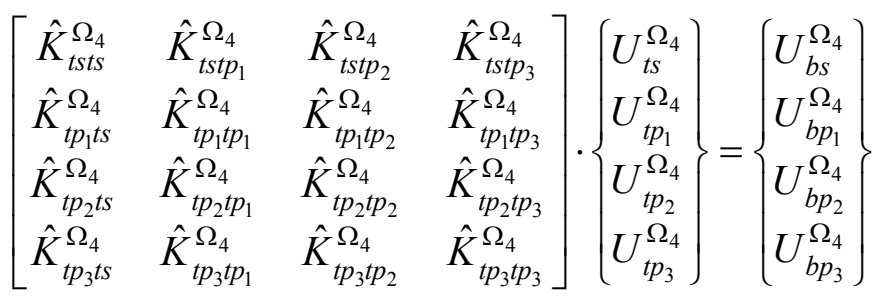


Aplicando-se a relação (3.91) na primeira, terceira, na quinta e na sétima relação de (3.84), tem-se:

$$
\begin{aligned}
& \widetilde{K}_{t s t s}^{\Omega_{4}}=K_{t s t s}^{\Omega_{4}}+K_{t s b s}^{\Omega_{4}} \cdot \hat{K}_{t s t s}^{\Omega_{4}}+K_{t s b p_{1}}^{\Omega_{4}} \cdot \hat{K}_{t p_{1} t s}^{\Omega_{4}}+K_{t s b p_{2}}^{\Omega_{4}} \cdot \hat{K}_{t p_{2} t s}^{\Omega_{4}}+K_{t s b p_{3}}^{\Omega_{4}} \cdot \hat{K}_{t p_{3} t s}^{\Omega_{4}} \\
& \widetilde{K}_{t s t p_{1}}^{\Omega_{4}}=K_{t s t p_{1}}^{\Omega_{4}}+K_{t s b s}^{\Omega_{4}} \cdot \hat{K}_{t s t p_{1}}^{\Omega_{4}}+K_{t s b p_{1}}^{\Omega_{4}} \cdot \hat{K}_{t p_{1} t p_{1}}^{\Omega_{4}}+K_{t s b p_{2}}^{\Omega_{4}} \cdot \hat{K}_{t p_{2} t p_{1}}^{\Omega_{4}}+K_{t s b p_{3}}^{\Omega_{4}} \cdot \hat{K}_{t p_{3} t p_{1}}^{\Omega_{4}} \\
& \widetilde{K}_{t s t p_{2}}^{\Omega_{4}}=K_{t s t p_{2}}^{\Omega_{4}}+K_{t s b s}^{\Omega_{4}} \cdot \hat{K}_{t s t p_{2}}^{\Omega_{4}}+K_{t s b p_{1}}^{\Omega_{4}} \cdot \hat{K}_{t p_{1} t p_{2}}^{\Omega_{4}}+K_{t s b p_{2}}^{\Omega_{4}} \cdot \hat{K}_{t p_{2} t p_{2}}^{\Omega_{4}}+K_{t s b p_{3}}^{\Omega_{4}} \cdot \hat{K}_{t p_{3} t p_{2}}^{\Omega_{4}} \\
& \widetilde{K}_{t s t p_{3}}^{\Omega_{4}}=K_{t s t p_{3}}^{\Omega_{4}}+K_{t s b s}^{\Omega_{4}} \cdot \hat{K}_{t s t p_{3}}^{\Omega_{4}}+K_{t s b p_{1}}^{\Omega_{4}} \cdot \hat{K}_{t p_{1} t p_{3}}^{\Omega_{4}}+K_{t s b p_{2}}^{\Omega_{4}} \cdot \hat{K}_{t p_{2} t p_{3}}^{\Omega_{4}}+K_{t s b p_{3}}^{\Omega_{4}} \cdot \hat{K}_{t p_{3} t p_{3}}^{\Omega_{4}} \\
& \widetilde{K}_{t p_{1} t s}^{\Omega_{4}}=K_{t p_{1} t s}^{\Omega_{4}}+K_{t p_{1} b s}^{\Omega_{4}} \cdot \hat{K}_{t s t s}^{\Omega_{4}}+K_{t p_{1} b p_{1}}^{\Omega_{4}} \cdot \hat{K}_{t p_{1} t s}^{\Omega_{4}}+K_{t p_{1} b p_{2}}^{\Omega_{4}} \cdot \hat{K}_{t p_{2} t s}^{\Omega_{4}}+K_{t p_{1} b p_{3}}^{\Omega_{4}} \cdot \hat{K}_{t p_{3} t s}^{\Omega_{4}} \\
& \widetilde{K}_{t p_{1} t p_{1}}^{\Omega_{4}}=K_{t p_{1} t p_{1}}^{\Omega_{4}}+K_{t p_{1} b s}^{\Omega_{4}} \cdot \hat{K}_{t s t p_{1}}^{\Omega_{4}}+K_{t p_{1} b p_{1}}^{\Omega_{4}} \cdot \hat{K}_{t p_{1} t p_{1}}^{\Omega_{4}}+K_{t p_{1} b p_{2}}^{\Omega_{4}} \cdot \hat{K}_{t p_{2} t p_{1}}^{\Omega_{4}}+K_{t p_{1} b p_{3}}^{\Omega_{4}} \cdot \hat{K}_{t p_{3} t p_{1}}^{\Omega_{4}} \\
& \widetilde{K}_{t p_{1} t p_{2}}^{\Omega_{4}}=K_{t p_{1} t p_{2}}^{\Omega_{4}}+K_{t p_{1} b s}^{\Omega_{4}} \cdot \hat{K}_{t s t p_{2}}^{\Omega_{4}}+K_{t p_{1} b p_{1}}^{\Omega_{4}} \cdot \hat{K}_{t p_{1} t p_{2}}^{\Omega_{4}}+K_{t p_{1} b p_{2}}^{\Omega_{4}} \cdot \hat{K}_{t p_{2} t p_{2}}^{\Omega_{4}}+K_{t p_{1} b p_{3}}^{\Omega_{4}} \cdot \hat{K}_{t p_{3} t p_{2}}^{\Omega_{4}} \\
& \widetilde{K}_{t p_{1} t p_{3}}^{\Omega_{4}}=K_{t p_{1} t p_{3}}^{\Omega_{4}}+K_{t p_{1} b s}^{\Omega_{4}} \cdot \hat{K}_{t s t p_{3}}^{\Omega_{4}}+K_{t p_{1} b p_{1}}^{\Omega_{4}} \cdot \hat{K}_{t p_{1} t p_{3}}^{\Omega_{4}}+K_{t p_{1} b p_{2}}^{\Omega_{4}} \cdot \hat{K}_{t p_{2} t p_{3}}^{\Omega_{4}}+K_{t p_{1} b p_{3}}^{\Omega_{4}} \cdot \hat{K}_{t p_{3} t p_{3}}^{\Omega_{4}} \\
& \widetilde{K}_{t p_{2} t s}^{\Omega_{4}}=K_{t p_{2} t s}^{\Omega_{4}}+K_{t p_{2} b s}^{\Omega_{4}} \cdot \hat{K}_{t s t s}^{\Omega_{4}}+K_{t p_{2} b p_{1}}^{\Omega_{4}} \cdot \hat{K}_{t p_{1} t s}^{\Omega_{4}}+K_{t p_{2} b p_{2}}^{\Omega_{4}} \cdot \hat{K}_{t p_{2} t s}^{\Omega_{4}}+K_{t p_{1} b p_{3}}^{\Omega_{4}} \cdot \hat{K}_{t p_{3} t s}^{\Omega_{4}} \\
& \widetilde{K}_{t p_{2} t p_{1}}^{\Omega_{4}}=K_{t p_{2} t p_{1}}^{\Omega_{4}}+K_{t p_{2} b s}^{\Omega_{4}} \cdot \hat{K}_{t s t p_{1}}^{\Omega_{4}}+K_{t p_{2} b p_{1}}^{\Omega_{3}} \cdot \hat{K}_{t p_{1} t p_{1}}^{\Omega_{4}}+K_{t p_{2} b p_{2}}^{\Omega_{4}} \cdot \hat{K}_{t p_{2} t p_{1}}^{\Omega_{4}}+K_{t p_{1} b p_{3}}^{\Omega_{4}} \cdot \hat{K}_{t p_{3} t p_{1}}^{\Omega_{4}} \\
& \widetilde{K}_{t p_{2} t p_{2}}^{\Omega_{4}}=K_{t p_{2} t p_{2}}^{\Omega_{4}}+K_{t p_{2} b s}^{\Omega_{4}} \cdot \hat{K}_{t s t p_{2}}^{\Omega_{4}}+K_{t p_{2} b p_{1}}^{\Omega_{4}} \cdot \hat{K}_{t p_{1} t p_{2}}^{\Omega_{4}}+K_{t p_{2} b p_{2}}^{\Omega_{4}} \cdot \hat{K}_{t p_{2} t p_{2}}^{\Omega_{4}}+K_{t p_{1} b p_{3}}^{\Omega_{4}} \cdot \hat{K}_{t p_{3} t p_{2}}^{\Omega_{4}} \\
& \widetilde{K}_{t p_{2} t p_{3}}^{\Omega_{4}}=K_{t p_{2} t p_{3}}^{\Omega_{4}}+K_{t p_{2} b s}^{\Omega_{4}} \cdot \hat{K}_{t s t p_{3}}^{\Omega_{4}}+K_{t p_{2} b p_{1}}^{\Omega_{4}} \cdot \hat{K}_{t p_{1} t p_{3}}^{\Omega_{4}}+K_{t p_{2} b p_{2}}^{\Omega_{4}} \cdot \hat{K}_{t p_{2} t p_{3}}^{\Omega_{4}}+K_{t p_{2} b p_{3}}^{\Omega_{4}} \cdot \hat{K}_{t p_{3} t p_{3}}^{\Omega_{4}} \\
& \widetilde{K}_{t p_{3} t s}^{\Omega_{4}}=K_{t p_{3} t s}^{\Omega_{4}}+K_{t p_{2} b s}^{\Omega_{4}} \cdot \hat{K}_{t s t s}^{\Omega_{4}}+K_{t p_{3} b p_{1}}^{\Omega_{4}} \cdot \hat{K}_{t p_{1} t s}^{\Omega_{4}}+K_{t p_{33} b p_{2}}^{\Omega_{4}} \cdot \hat{K}_{t p_{2} t s}^{\Omega_{4}}+K_{t p_{1} b p_{3}}^{\Omega_{4}} \cdot \hat{K}_{t p_{3} t s}^{\Omega_{4}} \\
& \widetilde{K}_{t p_{3} t p_{1}}^{\Omega_{4}}=K_{t p_{3} t p_{1}}^{\Omega_{4}}+K_{t p_{2} b s}^{\Omega_{4}} \cdot \hat{K}_{t s t p_{1}}^{\Omega_{4}}+K_{t p_{3} b p_{1}}^{\Omega_{4}} \cdot \hat{K}_{t p_{1} t p_{1}}^{\Omega_{4}}+K_{t p_{3} b p_{2}}^{\Omega_{4}} \cdot \hat{K}_{t p_{2} t p_{1}}^{\Omega_{4}}+K_{t p_{1} b p_{3}}^{\Omega_{4}} \cdot \hat{K}_{t p_{3} t p_{1}}^{\Omega_{4}} \\
& \widetilde{K}_{t p_{3} t p_{2}}^{\Omega_{4}}=K_{t p_{3} t p_{2}}^{\Omega_{4}}+K_{t p_{2} b s}^{\Omega_{4}} \cdot \hat{K}_{t s t p_{2}}^{\Omega_{4}}+K_{t p_{3} b p_{1}}^{\Omega_{4}} \cdot \hat{K}_{t p_{1} t p_{2}}^{\Omega_{4}}+K_{t p_{2} b p_{2}}^{\Omega_{4}} \cdot \hat{K}_{t p_{2} t p_{2}}^{\Omega_{4}}+K_{t p_{1} b p_{3}}^{\Omega_{4}} \cdot \hat{K}_{t p_{3} t p_{2}}^{\Omega_{4}} \\
& \widetilde{K}_{t p_{3} t p_{3}}^{\Omega_{4}}=K_{t p_{3} t p_{3}}^{\Omega_{4}}+K_{t p_{2} b s}^{\Omega_{4}} \cdot \hat{K}_{t s t p_{3}}^{\Omega_{4}}+K_{t p_{3} b p_{1}}^{\Omega_{4}} \cdot \hat{K}_{t p_{1} t p_{3}}^{\Omega_{4}}+K_{t p_{2} b p_{2}}^{\Omega_{4}} \cdot \hat{K}_{t p_{2} t p_{3}}^{\Omega_{4}}+K_{t p_{2} b p_{3}}^{\Omega_{4}} \cdot \hat{K}_{t p_{3} t p_{3}}^{\Omega_{4}}
\end{aligned}
$$

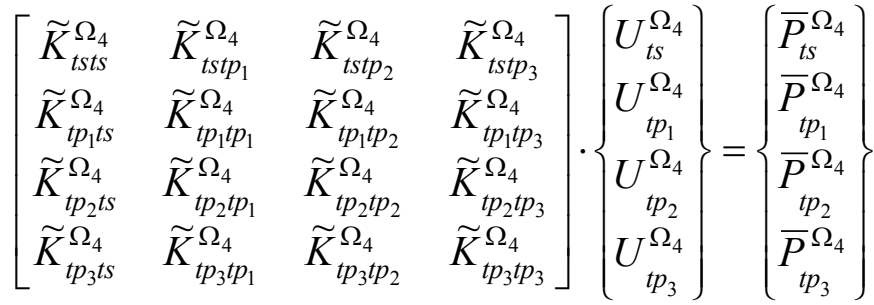


Com a expressão (3.96), conhecendo-se as forças de superfície, é possível resolver o sistema e obter os deslocamentos de superfície. Em seguida, calculam-se os deslocamentos e as forças de contato entre as camadas mediante o uso das expressões adequadas.

Ou seja, para este exemplo (figura 3.3) tem-se:

$4^{a}$ camada:

Conhecendo-se $\left\{U_{t}^{\Omega_{4}}\right\}$, obtêm-se $\left\{U_{b}^{\Omega_{4}}\right\}$ com (3.91) e $\left\{P_{b}^{\Omega_{4}}\right\}$ com (3.84).

$3^{a}$ camada:

Sabendo-se que $\left\{U_{t}^{\Omega_{3}}\right\}=\left\{U_{b}^{\Omega_{4}}\right\}$, obtêm-se $\left\{U_{b}^{\Omega_{3}}\right\}$ com (3.78), $\left\{P_{t}^{\Omega_{3}}\right\}$ e $\left\{P_{b}^{\Omega_{3}}\right\} \operatorname{com}(3.71)$.

$2^{a}$ camada:

Sabendo-se $\left\{U_{t}^{\Omega_{2}}\right\}=\left\{U_{b}^{\Omega_{3}}\right\}$, obtêm-se $\left\{U_{b}^{\Omega_{2}}\right\}$ com (3.67), $\left\{P_{t}^{\Omega_{2}}\right\}$ e $\left\{P_{b}^{\Omega_{2}}\right\} \operatorname{com}(3.62)$.

$1^{a}$ camada:

Sabendo-se que $\left\{U_{t}^{\Omega_{1}}\right\}=\left\{U_{b}^{\Omega_{2}}\right\}$, obtêm-se $\left\{P_{t}^{\Omega_{1}}\right\}$ e $\left\{P_{b}^{\Omega_{1}}\right\}$ com (3.61).

\subsection{Avaliação do número de operações envolvidas a priori}

Aparentemente, o procedimento empregado pela técnica da "rigidez sucessiva" poderia mostrar que é uma forma mais custosa em termos de operações envolvidas quando comparada pela técnica convencional de subregião. Isto, pois a primeira técnica exige a inversão e a multiplicação de muitas matrizes ao longo de cada estrato. E, para o caso de se ter elementos de fundação dentro de cada substrato, tem-se também a condensação de seus respectivos valores de deslocamentos, conforme apresentado no item 3.3.1. 
Assim, este item veio computar todas as etapas envolvidas em ambos os procedimentos. Sabendo-se que o número de operações em pontos flutuantes envolvidos nos seguintes procedimentos são:

$n^{3} \rightarrow$ produto entre duas matrizes quadradas de dimensões idênticas $n$;

$n \cdot m \cdot p \rightarrow$ produto entre duas matrizes retangulares de dimensões $\mathrm{A}(n, m)$ e $\mathrm{B}(m, p)$;

$4 / 3 n^{3} \rightarrow$ inversão de uma matriz de dimensão $n$;

$1 / 3 n^{3} \rightarrow$ resolução de sistema via Eliminação de Gauss com $n$ muito grande;

$n \cdot B^{2}-2 / 3 \cdot B^{3} \rightarrow$ resolução de sistema com matriz com comprimento de semibanda $B$.

Dimensão total das variáveis da superfície $n=3 \cdot N$, onde $\mathrm{N}$ é $\mathrm{O}$ número de nós de cada superfície, e $L$ é o número de camadas do maciço.

\section{Método da "rigidez sucessiva" (MRS):}

i) $\left[G^{i}\right]^{-1}: O(i)=4 / 3 \cdot n^{3} \cdot L=288 \cdot N^{3} \cdot L$

ii) $\left[G^{i}\right]^{-1} \cdot\left[H^{i}\right]: \quad O(i i)=n^{3} \cdot L=(6 N)^{3} \cdot L$

iii) Condensar variáveis do fuste: $O(i i i)=2 \cdot f(\alpha) \cdot\left[6 N+3 N_{\alpha}\right]^{3} \cdot L$

iv) Computar operações de equilíbrio entre solo/fundação - equações de 3.44.1 a 3.51.14. Neste caso, consideram-se as operações de produto de matrizes retangulares e a inversão de uma matriz $\left(\left[G_{f p_{i} f p_{i}}\right]\right)$ para cada elemento de fundação, adotando-se uma quantidade idêntica destes elementos dentro de cada estrato, num total de $N E$ por camada: $O(i v)=27 \cdot\left(N_{\alpha}\right)^{2} \cdot N E \cdot L \cdot\left[10 N+29,33 N_{\alpha}-3 N_{\alpha} / N E\right]$

v) $\left[K_{t s t s}^{\Omega_{i}}+K_{b s b s}^{\Omega_{i+1}}\right]^{-1}: O(v)=4 / 3 \cdot n^{3} \cdot(L-1)=36 \cdot N^{3} \cdot(L-1)$

vi) $\left[K_{t b}^{2}\right] \cdot\left(\left[K_{t t}^{1}\right]+\left[K_{b b}^{2}\right]\right)^{-1} \cdot\left[K_{b t}^{2}\right]: O(v i)=2 \cdot n^{3} \cdot(L-1)=2 \cdot(6 N)^{3} \cdot(L-1)$ 
vii) Resolver Sistema: $O($ vii $)=1 / 3 \cdot n^{3}=27 \cdot n^{3}$

Total de operações:

Sem elementos de fundação $(\alpha=0): \quad O(M R S)=N^{3} \cdot\{594 \cdot L-81\}$

Com elementos de fundação $(\alpha=1 / 3): \quad O(M R S)=N^{3} \cdot\{797 \cdot L+59 \cdot L \cdot N E-81\}$

\section{Sub-região convencional (SR):}

$B=9 \cdot N+\left(3 \cdot N_{\alpha}\right) \cdot N E$

$n=3 \cdot N \cdot(2 \cdot L)+3 \cdot N_{\alpha} \cdot(2 \cdot L) \cdot N E$

Total de operações:

Sem elementos de fundação $(\alpha=0): O(S R)=N^{3} \cdot(486 L-486)$

Com elementos de fundação $(\alpha=1 / 3)$ :

$$
O(S R)=2 \cdot N^{3} \cdot(9+N E)^{2} \cdot[(3+N E) \cdot L-1 / 3 \cdot(9+N E)]
$$

Onde $N_{\alpha}=\alpha \cdot N$, sendo $N_{\alpha}$ o número de nós de cada elemento de fundação e $\alpha$ é adotado com valor de 1/3. 


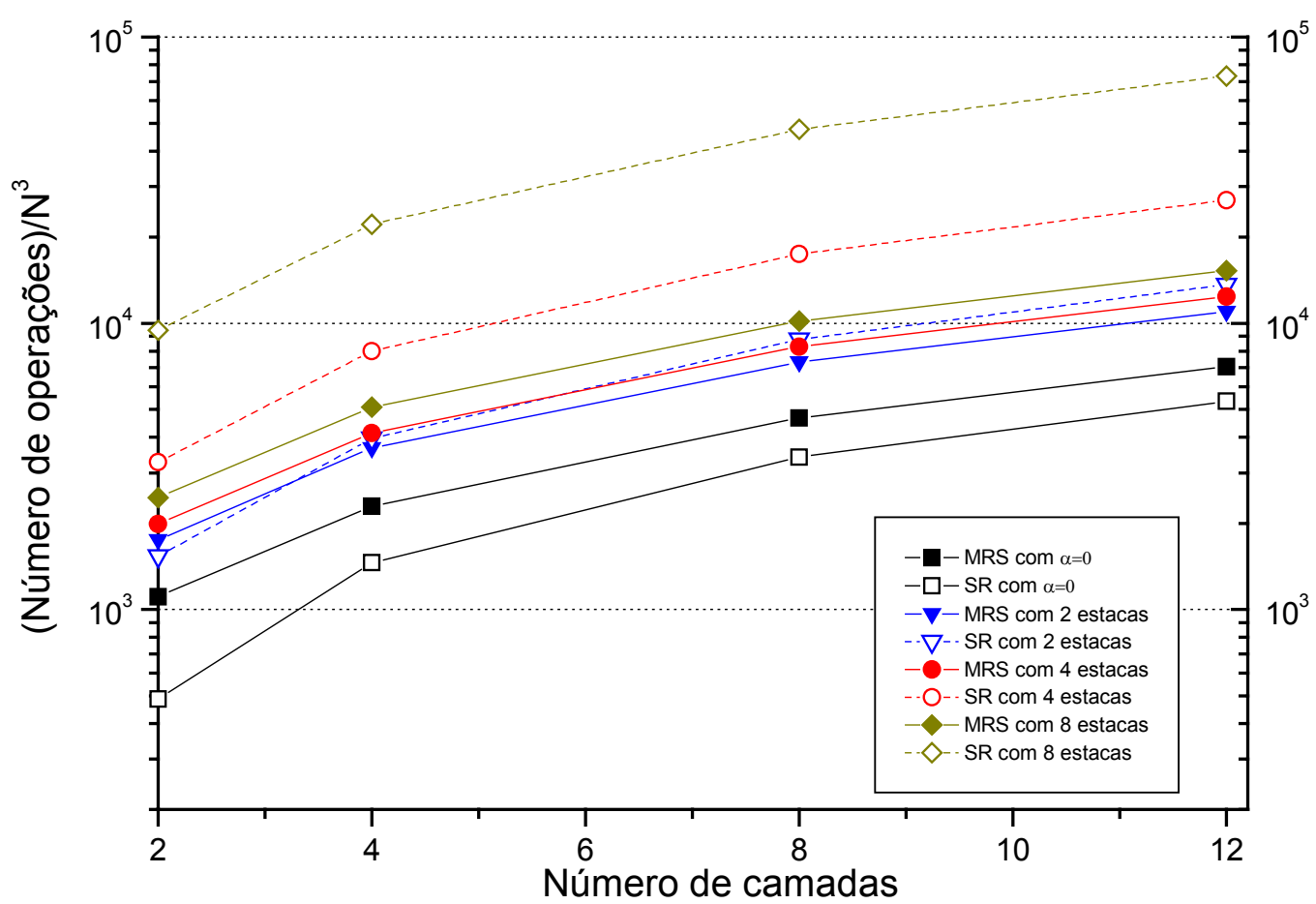

Figura 3.4 - Número de operações dividido por $\mathrm{N}^{3}$ versus número de camadas, por duas técnicas (escala Log): i) Método da "rigidez sucessiva" (MRS) ii) Método da sub-região (SR).

$\mathrm{Na}$ figura (3.4) são plotados os valores de operações envolvidas para a resolução de um meio estratificado com ou sem elemento enrijecedor. Nota-se que para o caso de se empregar o método da sub-região convencional sem a consideração dos elementos de fundação, o número de operações necessário para a montagem do problema são menores quando se emprega o método da "rigidez sucessiva". Entretanto, a partir do instante em que se consideram esses elementos, o método desenvolvido nesse trabalho demonstra ser bem mais eficiente.

Por fim, deve-se acrescentar que além da vantagem existente em relação ao parâmetro número de operações, uma outra grande vantagem desse método é a respeito do baixo custo exigido para o armazenamento da matriz final do sistema. Pois, por um lado, para a técnica da sub-região convencional é necessário armazenar a matriz final que trás informação explícita de todas as sub-regiões existentes no problema, enquanto que para o método da "rigidez sucessiva", chega-se a uma matriz final que contempla todas as influências das sub-regiões. 


\section{Capítulo 4}

\section{MÉTODO DOS ELEMENTOS FINITOS E ACOPLAMENTO MEC/MEF}

\subsection{Introdução}

Este capítulo tem o intuito de apresentar, de forma sucinta, o método dos elementos finitos (MEF), o qual será aplicado para a representação da superestrutura. Neste sentido, nos próximos itens são desenvolvidas as formulações elementares do MEF, comentando-se os elementos finitos empregados para a composição dos modelos laminares e das barras, e as referências bibliográficas que contêm a teoria dos elementos e seus códigos computacionais impressos.

$\mathrm{Na}$ segunda parte do capítulo é apresentada a técnica empregada para se realizar o acoplamento MEC/MEF, e por fim, são apresentados alguns exemplos para validar a formulação implementada.

\subsection{Formulação do método dos elementos finitos}

A superestrutura empregada neste trabalho é composta por um conjunto de lâminas (cascas e folhas poliédricas) e/ou elementos de barras, sendo eles formulados com a utilização do MEF na sua forma mais convencional, ou seja, usando o Método dos Deslocamentos. Isso, pois a aplicação do Método dos Elementos de Contorno para a formulação deste tipo de problema só é possível para alguns casos em que a estrutura laminar possua uma geometria particular, em virtude da complexidade matemática da obtenção das soluções fundamentais para casos genéricos. 
Em contrapartida, o MEF consiste na mais prática e poderosa ferramenta para a modelagem problemas de domínio finito de geometria quaisquer. O método consiste em subdividir o domínio do problema em subdomínios - chamados de elementos finitos - onde se aplicam as funções aproximadas para o campo das variáveis pertinentes às equações diferenciais parciais (EDPs) de um dado problema de valor de contorno (PVC). Para o caso de ele ser baseado no método dos deslocamentos, o campo aproximado é o dos deslocamentos, sendo estes associados a parâmetros nodais.

As equações aproximadoras são, então, integradas em cada elemento via aplicação das técnicas variacionais ou dos resíduos ponderados, já se levando em consideração os campos aproximados, as relações constitutivas e cinemáticas do modelo mecânico. Esse procedimento é feito, elemento-a-elemento, em um sistema de referência local arbitrário de cada subdomínio, os quais são interligados pelos valores nodais já rotacionados para um sistema de referência global.

O MEF aplicado a problemas da elasticidade tridimensional pode ser formulado usando-se, por exemplo, o princípio dos trabalhos virtuais (PTV).

Partindo-se então das equações diferenciais de equilíbrio:

$$
\sigma_{i j, j}+b_{i}=0 \quad i, j=1,2,3
$$

onde $b_{i}$ são as forças volumétricas por unidade de volume e $\sigma_{i j}$, as 6 componentes de tensões independentes obtidas de um elemento infinitesimal, uma vez que existe simetria das tensões de cisalhamento, obtida levando-se em consideração a ausência de momentos concentrados neste elemento e para casos onde a tensão não seja produzida por campos magnéticos e elétricos.

As componentes de tensões são projetadas em um contorno infinitesimal $d \Gamma$ e produzem forças de superfície - $p_{i}$ - correlacionadas com as tensões mediante a relação: 


$$
p_{i}=\sigma_{i j} \cdot n_{j} \quad i, j=1,2,3
$$

onde $n_{j}$ são os co-senos diretores normais ao contorno $d \Gamma$.

As forças externas $\left(\bar{p}_{i}\right)$ aplicadas no elemento, chamadas condições naturais de contorno ou condição de fronteira de Neumann, agem numa região denominada de $\Gamma_{p}$ e devem ser equilibradas pelas forças de superfície de (4.2) nesta região, ou seja:

$$
\bar{p}_{i}=p_{i}=\sigma_{i j} \cdot n_{j} \quad \text { em } \quad \Gamma_{p} \quad i, j=1,2,3
$$

As condições geométricas, que são condições chamadas essenciais, ou condições de fronteira de Dirichlet, estão prescritas na região $\Gamma_{u}$, e ficam

$$
\bar{u}_{i}=u_{i} \quad \text { em } \quad \Gamma_{u} \quad i, j=1,2,3
$$

sendo que a superfície total do contorno é dada por $\Gamma=\Gamma_{p}+\Gamma_{u}$.

Pode-se aplicar o método dos resíduos ponderados na equação de equilíbrio (4.1), onde a função peso é escolhida como sendo um campo de deslocamentos virtuais, que são perturbações impostas na posição de equilíbrio. Assim, considere-se, então, um campo de deslocamentos contínuo e virtual - $\widehat{u}_{i}$ - que satisfaça:

$$
\widehat{u}_{i}=0 \quad \text { em } \quad \Gamma_{u} \quad i, j=1,2,3
$$

Impondo-se na equação (4.1) a função peso na forma de integral, tem-se:

$$
\int_{V}\left(\sigma_{i j, j}+b_{i}\right) \hat{u}_{i} d V=0 \quad i, j=1,2,3
$$

E uma vez que $\widehat{u}_{i}$ é arbitrário, a equação (4.6) é satisfeita quando o termo em parênteses é nulo. Assim, esta expressão é idêntica a (4.1). 
Usando-se a identidade matemática

$$
\left(\sigma_{i j} \hat{u}_{i}\right)_{, j}=\sigma_{i j, j} \cdot \hat{u}_{i}+\sigma_{i j} \cdot \widehat{u}_{i, j} \quad i, j=1,2,3
$$

a relação (4.6) pode ser reescrita como:

$$
\int_{V}\left[\left(\sigma_{i j} \cdot \hat{u}_{i}\right)_{, j}-\sigma_{i j} \cdot \hat{u}_{i, j}+b_{i} \cdot \hat{u}_{i}\right] d V=0 \quad i, j=1,2,3
$$

Aplicando-se o teorema da divergência na equação (4.8), e considerando-se (4.3) e (4.5), tem-se:

$$
\int_{V}-\left(\sigma_{i j} \cdot \widehat{u}_{i, j}+b_{i} \cdot \widehat{u}_{i}\right) d V+\int_{\Gamma_{p}} p_{i} \cdot \widehat{u}_{i} d S=0 \quad i, j=1,2,3
$$

Pela simetria dos tensores de tensões $\left(\sigma_{i j}=\sigma_{j i}\right)$ e empregando-se o tensor de deformação de Cauchy, o qual se refere às deformações homogêneas (lineares), chega-se a :

$$
\sigma_{i j} \cdot \hat{u}_{i, j}=1 / 2 \sigma_{i j}\left[\hat{u}_{i, j}+\widehat{u}_{j, i}\right]=\sigma_{i j} \cdot \widehat{\varepsilon}_{i j}
$$

onde $\widehat{\varepsilon}$ são as deformações virtuais advindas dos deslocamentos virtuais $\hat{u}$. Portanto, a equação (4.9) leva a:

$$
\int_{V} \hat{\varepsilon}^{T} \cdot \sigma d V=\int_{V} \hat{u} \cdot b d V+\int_{S_{\Gamma_{p}}} \hat{u} \cdot \bar{p} d S
$$

onde o primeiro termo da relação (4.11) é relacionado ao trabalho virtual interno, enquanto os outros dois termos se relacionam ao trabalho virtual devido às forças externas. Para o último termo da expressão (4.11), tem-se que impor condições de contorno naturais, ou de forças.

A relação (4.11) independe das características do material e podem ser escritas em função dos deslocamentos ou de tensões. Para expressá-la em termos de deslocamentos, é necessário fazer uso das relações constitutivas do material. Assim, a relação entre tensão e deformação é expressa por: 


$$
\sigma=D \varepsilon
$$

onde $D$ é um campo tensorial de quarta ordem que compõe as características do material e possui 36 constantes independentes. Para o caso de o material ser elástico e linear, D é constante, e considerando-se as diferentes classes de simetria existentes em um elemento infinitesimal, o meio é denominado de isótropo, e chega-se então a 2 constantes independentes do material: o módulo de elasticidade longitudinal $(E)$ e o coeficiente de Poisson ( $v)$.

A relação (4.11), que representa o Princípio dos Trabalhos Virtuais (PTV), apresenta dois espaços de funções. A primeira representa o espaço das tensões admissíveis, e a segunda o espaço das funções pesos. $\mathrm{Na}$ mecânica dos sólidos, é comum empregar o famoso método de BubnovGalerkin no PTV, o qual impõe que as funções pesos e admissíveis são iguais. Assim, os campos de deslocamentos de ambas as funções são aproximados por funções interpoladoras via parâmetros nodais, sendo esta expressa por:

$$
u=H\left(x_{i}\right) u^{\text {nodal }} \quad i=1,2,3
$$

E, para as deformações, tem-se:

$$
\varepsilon=B\left(x_{i}\right) u^{\text {nodal }} \quad i=1,2,3
$$

onde $\mathrm{H}$ e $\mathrm{B}$ são as matrizes de interpolação de deslocamentos e deslocamento-deformação.

Assim, para um elemento finito genérico "e", a relação (4.11) pode ser reescrita mediante o emprego das expressões (4.13) e (4.14) como:

$$
\begin{aligned}
& \left\{\hat{u}_{e}^{\text {nodal }}\right\}^{T}\left[\int_{V} B_{e}^{T} D_{e} B_{e} d V\right] \cdot \hat{u}_{e}^{\text {nodal }}=\left\{\hat{u}_{e}^{\text {nodal }}\right\}^{T} \int_{V} H_{e}^{T} b_{e} d V+ \\
& \left\{\hat{u}_{e}^{\text {nodal }}\right\}^{T} \int_{S_{\Gamma_{p}}} H_{e}^{T} \bar{p}_{e} d S
\end{aligned}
$$


Então, após impor campos de deslocamentos virtuais unitários independentes, a relação (4.15) resulta para um elemento em:

$$
K_{e} \cdot u_{e}^{\text {nodal }}=F_{e}
$$

onde $K_{e}$ é a matriz de rigidez, $F_{e}$ é o vetor resultante devido às forças externas aplicadas e $u_{e}$ é o vetor dos deslocamentos nodais do elemento "e". Fazendo-se o somatório de todos os elementos "ne" que compõem o domínio em estudo, chega-se a:

$$
\begin{gathered}
K=\sum_{e=1}^{n e}\left[\int_{V_{e}} B_{e}^{T} D_{e} B_{e} d V\right] \\
F=\sum_{e=1}^{n e}\left[\int_{V_{e}} H_{e}^{T} b_{e} d V+\int_{S_{\Gamma_{p}}} H_{e}^{T} \bar{p}_{e} d S\right]
\end{gathered}
$$

Portanto,

$$
K \cdot u^{\text {nodal }}=F
$$

onde $u^{\text {nodal }}$ é $\mathrm{o}$ vetor das incógnitas dos deslocamentos nodais correspondentes ao número total de graus de liberdade do domínio.

\subsection{Superestrutura formada por elementos laminares}

Entende-se por elementos laminares aqueles em que uma dimensão é muito menor que as outras duas, e, quando uma estrutura é formada por uma ou mais lâminas com superfícies planas, é chamada de folhas poliédricas. As caixas d'água, os núcleos de rigidez de edifícios, as vigas paredes e silos são alguns exemplos destes tipos de estruturas.

Para a simulação da superestrutura com elementos laminares, utilizou-se a sobreposição de duas formulações independentes: uma para representar o efeito de membrana, e outra para o efeito de placa. 
Neste contexto, será apresentado o elemento que foi utilizado para modelar as superfícies bidimensionais a fim de formar o elemento laminar. Entretanto, em função da existência de trabalhos que já detalharam a formulação tanto do elemento de membrana quanto de placa, este capítulo não entrará no mérito da formulação, citando apenas os trabalhos existentes para seu melhor entendimento.

As hipóteses admitidas para a formação do elemento laminar deste trabalho são as hipóteses de Kirchhoff-Love:

- A espessura da lâmina é pequena em relação às dimensões e aos raios de curvatura da superfície média;

- As tensões normais à superfície média são desprezíveis em relação às demais;

- Os pontos pertencentes a uma mesma reta normal à superfície média na situação indeformada encontram-se em uma mesma reta à superfície deformada;

- Os deslocamentos são muito pequenos em relação à espessura.

Com estas hipóteses, o campo de deslocamento para uma área infinitesimal de um elemento laminar é dado por:

$$
\{u\}=\left\{\begin{array}{l}
u\left(x_{1}, x_{2}, x_{3}\right) \\
v\left(x_{1}, x_{2}, x_{3}\right) \\
w\left(x_{1}, x_{2}, x_{3}\right)
\end{array}\right\}=\left\{\begin{array}{c}
u_{0}\left(x_{1}, x_{2}\right)-x_{3} d w_{0} / d x_{1} \\
v_{0}\left(x_{1}, x_{2}\right)-x_{3} d w_{0} / d x_{2} \\
w_{0}\left(x_{1}, x_{2}\right)
\end{array}\right\}
$$

onde $u_{0}, v_{0}$ e $w_{0}$ são os campos de deslocamentos locais de um ponto genérico considerado, respectivamente, nas direções $x_{1}, x_{2}$ e $x_{3}$.

O campo de pequenas deformações fica definido pelo campo de deformação do efeito de membrana e do efeito de flexão mediante a relação seguinte:

$$
\{\varepsilon\}=\{\varepsilon\}_{m}+\{\varepsilon\}_{f}=\left\{\begin{array}{c}
\varepsilon_{1} \\
\varepsilon_{2} \\
\gamma_{12}
\end{array}\right\}=\left\{\begin{array}{c}
d u_{0} / d x_{1} \\
d v_{0} / d x_{2} \\
d u_{0} / d x_{2}+d v_{0} / d x_{1}
\end{array}\right\}-x_{3} \cdot\left\{\begin{array}{c}
d^{2} w_{0} / d x_{1}^{2} \\
d^{2} w_{0} / d x_{2}^{2} \\
2 \cdot d^{2} w_{0} / d x_{1} \cdot d x_{2}
\end{array}\right\}
$$


O primeiro vetor da expressão à direita de (4.21) se refere ao efeito de membrana, e a segunda parcela ao efeito de flexão, onde " $m$ " e "f" são abreviações de membrana e flexão, respectivamente.

Desta forma, o campo de tensões é expresso pela relação constitutiva (4.12), que para este caso é dada por:

$$
\{\sigma\}=[D] \cdot\{\varepsilon\}=[D] \cdot\left(\{\varepsilon\}_{m}+\{\varepsilon\}_{f}\right)=\{\sigma\}_{m}+\{\sigma\}_{f}
$$

O campo de deslocamentos na formulação do MEF para o elemento laminar fica então:

$$
\{u\}=[\varphi] \cdot\left\{u^{\text {nodal }}\right\}=\left[\begin{array}{cc}
{[\varphi]_{m}} & {[0]} \\
{[0]} & {[\varphi]_{f}}
\end{array}\right] \cdot\left\{\begin{array}{l}
\left\{u^{\text {nodal }}\right\}_{m} \\
\left\{u^{\text {nodal }}\right\}_{f}
\end{array}\right\}
$$

de modo que o vetor $\left\{\mathrm{u}^{\text {nodal }}\right\}$ representa os deslocamentos nodais de um elemento genérico no sistema local. Expandindo-se este vetor de deslocamento, tém-se para um ponto "i" do elemento:

$$
\begin{gathered}
\{u\}_{m}^{T}=\left\{\begin{array}{llll}
u_{i} & v_{i} & \theta_{i} \cdots
\end{array} \quad i=1, \ldots .,\right. \text { pontos nodais do elemento } \\
\{\delta\}_{f}^{T}=\left\{\begin{array}{lll}
w_{i} & \theta_{x_{1} i} & \theta_{x_{2}} \\
\cdots
\end{array}\right\} i=1, \ldots ., \text { pontos nodais do elemento }
\end{gathered}
$$

E as submatrizes $[\varphi]_{m}$ e $[\varphi]_{\mathrm{f}}$ são as funções de forma para a parcela de membrana e de flexão.

A relação (4.14) aplicada na associação dos dois efeitos leva ao campo de deformação expresso por:

$$
\{\varepsilon\}=\left[[B]_{m}+x_{3} \cdot[B]_{f}\right] \cdot\left\{u^{\text {nodal }}\right\}=[B] \cdot\left\{u^{\text {nodal }}\right\}
$$

onde $[B]_{m}$ e $[B]_{f}$ são as matrizes de interpolação de deslocamentodeformação correspondentes aos estados de membrana e flexão.

A matriz de rigidez do elemento híbrido pode ser obtida com 0 emprego da relação (4.15) levando-se em conta as equações (4.22) e (4.26). Assim, tem-se: 


$$
\begin{aligned}
& {[K]=\sum_{e=1}^{n e}\left[\int_{V_{e}} B_{e}^{T} D_{e} B_{e} d V\right]=} \\
& \sum_{e=1}^{n e}\left[\int_{V}\left\{\begin{array}{c}
{[B]_{m}^{T}} \\
x_{3} \cdot[B]_{f}^{T}
\end{array}\right\} \cdot\left[\begin{array}{cc}
{[D]_{m}} & {[D]_{m f}} \\
{[D]_{f m}} & {[D]_{f}}
\end{array}\right] \cdot\left\{[B]_{m}^{T} \quad x_{3} \cdot[B]_{f}^{T}\right\} d V\right]
\end{aligned}
$$

Matricialmente, a expressão (4.27) pode ser representada por:

$$
[K]=\left[\begin{array}{ll}
{[K]_{m}} & {[K]_{m f}} \\
{[K]_{f m}} & {[K]_{f}}
\end{array}\right]
$$

A submatriz $[\mathrm{K}]_{\mathrm{mf}}$ é nula, pois ela pode ser escrita como:

$$
[K]_{m f}=\int[B]_{m}^{T} \cdot x_{3} \cdot[D] \cdot[B]_{f} d V=\int[B]_{m}^{T} \cdot[D]_{m f} \cdot[B]_{f} d V
$$

e integrando-se a matriz constitutiva ao longo da espessura $h$, e considerando-se que o material seja homogêneo ou com variação simétrica em relação ao plano médio:

$$
[D]_{m f}=\int_{-h / 2}^{h / 2} x_{3} \cdot[D] d x_{3}=[0]
$$

Da mesma forma, a matriz $[D]_{\mathrm{fm}}$ é nula. A independência entre os campos que contemplam o efeito de membrana e de flexão ocorre para esta formulação. Assim, a matriz de rigidez fica:

$$
[K]=\left[\begin{array}{cc}
{[K]_{m}} & {[0]} \\
{[0]} & {[K]_{f}}
\end{array}\right]
$$

\subsubsection{Efeito de membrana}

O elemento de membrana utilizado foi o desenvolvido por BERGAN \& FELIPPA (1985), que utilizam a formulação livre. Desta forma, em seu trabalho é apresentada toda a formulação para a sua obtenção. No departamento de Estruturas da Escola de Engenharia de São Carlos (EESC), foram desenvolvidos os trabalhos de PETELEIRO (1996) e MESQUITA (1998), que também abordam de maneira bem clara a teoria 
para o desenvolvimento deste elemento bem como apresenta exemplos para validação da formulação.

Não se pretende desenvolver esta formulação neste trabalho, uma vez que ela não é o objeto principal do projeto. Apresenta-se um desenho esquemático do elemento com seus graus de liberdade.

\subsubsection{Efeito de flexão}

O elemento utilizado para a avaliação do efeito de flexão é o conhecido DKT. Da mesma forma que fora feito para o elemento de membrana, não se tem o intuito aqui de apresentar a formulação deste elemento. Em BATOZ (1980), é desenvolvida toda a formulação deste elemento bem como o código computacional para a montagem da matriz de rigidez e do vetor de forças equivalentes. Na figura 4.1, apresenta-se esquematicamente a geometria e as variáveis nodais do elemento já incorporando o efeito de membrana.

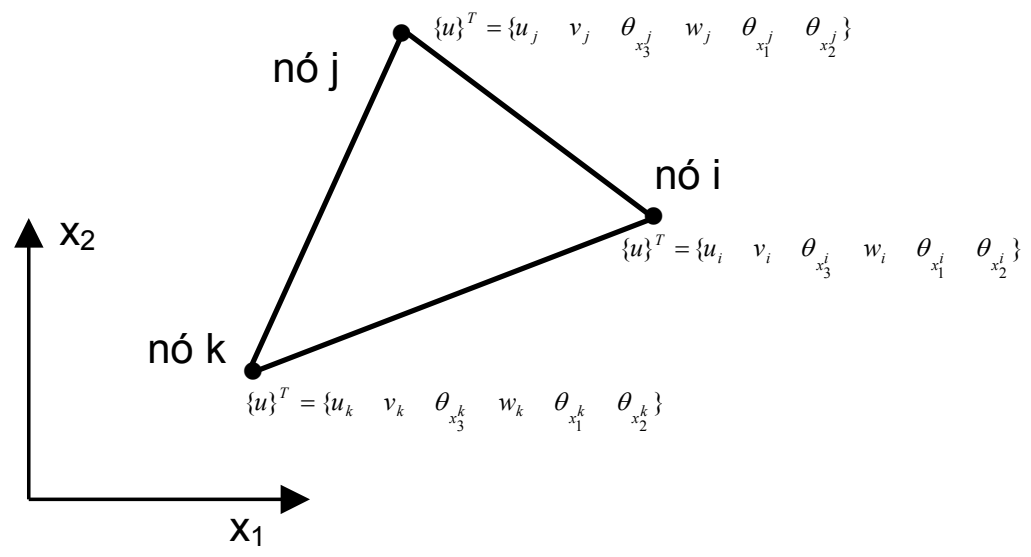

Figura 4.1 - Geometria e os 18 graus de liberdade do elemento laminar da junção DKT/FF no sistema local $O \mathrm{x}_{1} \mathrm{x}_{2}$.

\subsubsection{Matriz de rigidez do elemento laminar no plano tridimensional}

Para a geração da matriz de rigidez de um elemento plano no espaço tridimensional é necessário rotacionar esta matriz local para um sistema de referência global (vide figura 4.2). Esta transformação de sistema local para global se dá da seguinte forma: 


$$
\begin{gathered}
{[K]_{\text {global }}=\sum_{i=1}^{n e}[\beta]_{i}^{T} \cdot[K] \cdot[\beta]_{i}} \\
\{f\}_{\text {global }}=\sum_{i=1}^{n e}[\beta]_{i}^{T} \cdot\{f\}_{i} \\
\{u\}_{e}^{\text {local }}=[\beta]_{e} \cdot\{u\}_{e} \\
{[\sigma]_{e}^{\text {local }}=[D]_{e} \cdot[B]_{e}^{\text {local }} \cdot\{u\}_{e}^{\text {local }}}
\end{gathered}
$$

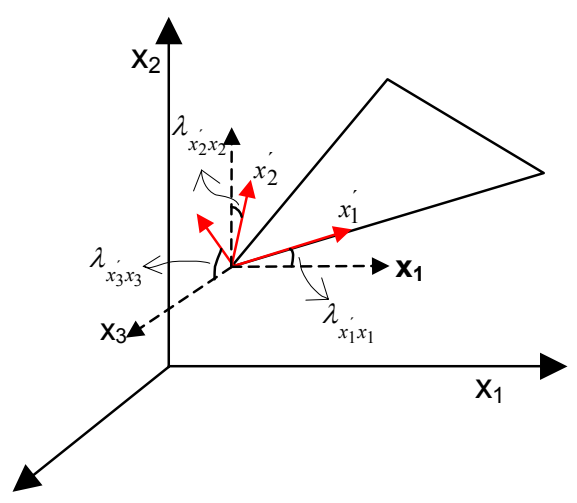

$x_{1}^{\prime}$ e $x_{2}^{\prime}$ no plano

do elemento

Figura 4.2 - Relação entre sistema local e global de um plano qualquer no espaço 3D.

A matriz de transformação $[\beta]_{\mathrm{e}}$ representa os co-senos diretores do elemento "e".

Os eixos locais podem ser tomados em qualquer posição dentro do plano do elemento, uma vez que o elemento é definido em um plano. Usualmente, os eixos locais são obtidos a partir da direção de um lado do elemento. Neste trabalho, o sistema local " $x_{1}^{\prime}$ " parte do nó local um (1) até o nó dois (2), e os eixos " $x_{2}^{\prime} " \mathrm{e} " x_{3}^{\prime} "$ são obtidos por relações geométricas. Esta matriz de transformação relaciona as coordenadas locais com as globais mediante a relação:

$$
\left\{x_{i}^{\prime}\right\}=[\bar{\beta}] \cdot\left\{x_{i}\right\}_{\text {global }} \quad i=1,2,3
$$

sendo que 


$$
\left\{x^{\prime}\right\}=\left\{\begin{array}{l}
x_{1}^{\prime} \\
x_{2}^{\prime} \\
x_{3}^{\prime}
\end{array}\right\}
$$

e, como as translações e as rotações são campos independentes vetorialmente, a matriz de transformação [ $\beta]$ fica:

$$
[\beta]=\left[\begin{array}{cc}
- & \\
{[\bar{\beta}]_{3 \times 3}} & {[0]} \\
{[0]} & {[\bar{\beta}]_{3 \times 3}}
\end{array}\right]_{6 x 6}
$$

sendo que $[\bar{\beta}]$ é dado por:

$$
\overline{[\beta}]=\left[\begin{array}{lll}
\lambda_{x_{1} x_{1}} & \lambda_{x_{1} x_{2}} & \lambda_{x_{1} x_{3}} \\
\lambda_{x_{2} x_{1}} & \lambda_{x_{2} x_{2}} & \lambda_{x_{2} x_{3}} \\
\lambda_{x_{3} x_{1}} & \lambda_{x_{3} x_{2}} & \lambda_{x_{3} x_{3}}^{\prime}
\end{array}\right]
$$

onde $\lambda_{x_{i}^{\prime} x_{i}}$ representa o co-seno diretor entre o eixo local $x_{i}^{\prime}$ e o eixo global $x_{i}$ na direção " $i$ ".

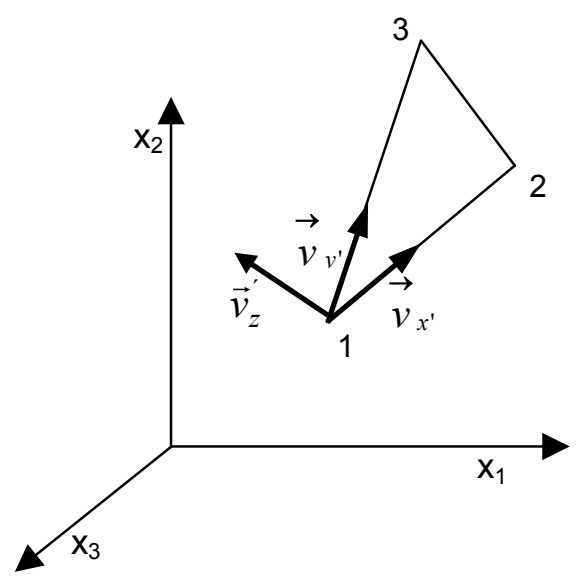

Figura 4.3 - Vetores auxiliares para cálculo dos co-senos diretores de um elemento.

Para o cálculo desses co-senos, são utilizados artifícios da geometria analítica. Para isto tem-se o vetor formado pela reta do lado 1-2 do elemento 
em uma posição do espaço 3D qualquer (ver figura 4.3). Este vetor pode ser representado por:

$$
\vec{v}_{x^{\prime}}=\left(x_{1}^{2}-x_{1}^{1}\right) \cdot \vec{i}+\left(x_{2}^{2}-x_{2}^{1}\right) \cdot \vec{j}+\left(x_{3}^{2}-x_{3}^{1}\right) \cdot \vec{k}
$$

onde $x_{i}^{j}$ representa a coordenada cartesiana no sistema global da direção "i" do nó local "j" de um elemento genérico.

Normalizando-se a equação (4.40), tem-se:

$$
\vec{v}_{x^{\prime}}=\frac{v_{x^{\prime}}}{\left\|\vec{v}_{x^{\prime}}\right\|}=\frac{1}{L_{12}} \cdot\left\{\begin{array}{l}
x_{1}^{2}-x_{1}^{1} \\
x_{2}^{2}-x_{2}^{1} \\
x_{3}^{2}-x_{3}^{1}
\end{array}\right\}=\left\{\begin{array}{l}
\lambda_{x_{1} x_{1}} \\
\lambda_{x_{1} x_{2}} \\
\lambda_{x_{1} x_{3}}
\end{array}\right\}^{T} \cdot\left\{\begin{array}{l}
\vec{i} \\
\vec{j} \\
\vec{k}
\end{array}\right\}
$$

onde

$$
L_{12}=\sqrt{\left(x_{1}^{2}-x_{1}^{1}\right)^{2}+\left(x_{2}^{2}-x_{2}^{1}\right)^{2}+\left(x_{3}^{2}-x_{3}^{1}\right)^{2}}
$$

O vetor normal ao plano do elemento pode ser obtido mediante 0 produto vetorial entre $\vec{v}_{x^{\prime}}$ e $\vec{v}_{y^{\prime}}$, onde $\vec{v}_{y^{\prime}}$ é dado por:

$$
\vec{v}_{y^{\prime}}=\left(x_{1}^{3}-x_{1}^{1}\right) \cdot \vec{i}+\left(x_{2}^{3}-x_{2}^{1}\right) \cdot \vec{j}+\left(x_{3}^{3}-x_{3}^{1}\right) \cdot \vec{k}
$$

Assim, de (4.41) e (4.43), obtém-se:

$$
\overrightarrow{V_{z^{\prime}}}=\overrightarrow{v_{x^{\prime}}} \wedge \overrightarrow{v_{y^{\prime}}} \stackrel{\text { normalizando }}{\longrightarrow} \overrightarrow{v_{z^{\prime}}}=\frac{\left(\vec{v}_{x^{\prime}} \wedge \vec{v}_{y^{\prime}}\right)}{\left.\| \vec{v}_{x^{\prime}} \wedge \overrightarrow{v_{y^{\prime}}}\right) \|}
$$

de modo que:

$$
\vec{v}_{z^{\prime}}=\frac{1}{\sqrt{\left(\Delta x_{2} x_{3}\right)^{2}+\left(\Delta x_{1} x_{3}\right)^{2}+\left(\Delta x_{1} x_{2}\right)^{2}}} \cdot\left\{\begin{array}{l}
\Delta x_{2} x_{3} \\
\Delta x_{1} x_{3} \\
\Delta x_{1} x_{2}
\end{array}\right\}=\left\{\begin{array}{l}
\lambda_{x_{3} x_{1}} \\
\lambda_{x_{3} x_{2}} \\
\lambda_{x_{3} x_{3}}^{\prime}
\end{array}\right\}^{T} \cdot\left\{\begin{array}{l}
\vec{i} \\
\vec{j} \\
\vec{k}
\end{array}\right\}
$$


onde

$$
\begin{aligned}
& \Delta x_{1} x_{2}=\left(x_{1}^{2}-x_{1}^{1}\right) \cdot\left(x_{2}^{3}-x_{2}^{1}\right)-\left(x_{2}^{2}-x_{2}^{1}\right) \cdot\left(x_{1}^{3}-x_{1}^{1}\right) \\
& \Delta x_{2} x_{3}=\left(x_{2}^{2}-x_{2}^{1}\right) \cdot\left(x_{3}^{3}-x_{3}^{1}\right)-\left(x_{3}^{2}-x_{3}^{1}\right) \cdot\left(x_{2}^{3}-x_{2}^{1}\right) \\
& \Delta x_{1} x_{3}=\left(x_{3}^{2}-x_{3}^{1}\right) \cdot\left(x_{1}^{3}-x_{1}^{1}\right)-\left(x_{1}^{2}-x_{1}^{1}\right) \cdot\left(x_{3}^{3}-x_{3}^{1}\right)
\end{aligned}
$$

E, finalmente:

$$
\overrightarrow{v_{y^{\prime}}}=\overrightarrow{v_{x^{\prime}}} \wedge \overrightarrow{v_{y^{\prime}}}
$$

De modo que se chega a:

$$
\vec{v}_{y^{\prime}}=\left\{\begin{array}{l}
\lambda_{x_{3} x_{2}} \cdot \lambda_{x_{1} x_{3}}-\lambda_{x_{3} x_{3}} \cdot \lambda_{x_{1} x_{2}} \\
\lambda_{x_{3} x_{3}}^{\prime} \cdot \lambda_{x_{1} x_{1}}-\lambda_{x_{3} x_{1}}^{\prime} \cdot \lambda_{x_{1} x_{3}}^{\prime} \\
\lambda_{x_{3} x_{1}}^{\prime} \cdot \lambda_{x_{1} x_{2}}-\lambda_{x_{3} x_{2}}^{\prime} \cdot \lambda_{x_{1} x_{1}}^{\prime}
\end{array}\right\}=\left\{\begin{array}{l}
\lambda_{x_{2} x_{1}} \\
\lambda_{x_{2} x_{2}} \\
\lambda_{x_{2} x_{3}}^{\prime}
\end{array}\right\}
$$

Com as matrizes de rigidezes locais obtidas nos trabalhos de referência (BERGAN \& FELIPPA (1985) e BATOZ (1980)) para se considerar o efeito de membrana e flexão e a matriz de transformação obtida mediante as expressões (4.41), (4.45) e (4.48), pode-se montar a matriz e o vetor de forças equivalentes para formar o elemento laminar, que é denominado abreviadamente de DKTFF.

\subsection{Superestrutura formada por edifícios 3D sujeitos às ações verticais e horizontais}

Os edifícios são modelados empregando-se os elementos finitos de barras tridimensionais para se representar os elementos lineares de vigas e pilares, sem se considerar nestes o efeito de torção. A laje é considerada como se fosse um diafragma infinitamente rígido em seu próprio plano horizontal, e por isso os deslocamentos horizontais de cada pavimento $\left(\begin{array}{lll}u, v & e & \theta_{x_{3}}\end{array}\right)$ são iguais. Portanto, transferem-se todas as influências dos pilares e vigas de cada andar para um nó mestre que é o centro de torção do 
pavimento tipo. Esse modelo de edifício leva a uma sensível redução dos graus de liberdade do sistema final.

As ações verticais são aplicadas nas vigas, podendo ser pontuais ou distribuídas, e as ações horizontais devidas ao efeito de vento - forças horizontais e torcionais - devem ser prescritas no centro de torção pontualmente para cada pavimento.

Os nós dos pilares do andar térreo são ligados com os nós da lâmina nos pontos de contato.

Os trabalhos que apresentam a teoria completa da formulação do edifício utilizado são de RIOS (1991) e BEZERRA (1995).

\subsection{Acoplamento MEC/MEF}

A formulação advinda do MEC e a do MEF podem ser acopladas mediante a consideração das condições de equilíbrio e compatibilidade existentes nos pontos nodais em comum aos dois métodos. Assim, a expressão final obtida em (3.60) que mostra toda a influência do solo nãohomogêneo, enrijecido ou não por estaca, pode ser relacionada com a equação (4.19) obtida pelo MEF.

Entretanto, a representação das forças externas no MEC é tomada como forças de superfície, ou seja, forças por unidade de área, enquanto que as ações externas formuladas pelo MEF empregam comumente o conceito de força nodal equivalente, ou seja, forças concentradas.

Neste contexto, para o devido acoplamento entre as duas formulações, é necessário empregar um campo de forças comum ao dois métodos. Ou se representam as forças de superfície em forças nodais equivalentes ou vice-versa.

Para a presente formulação do conjunto solo-estrutura, o único fator de influência para a melhor decisão sobre qual procedimento empregar é respeito ao número de operações envolvidas para a conversão de uma ou outra formulação. Pretende-se, neste trabalho, transformar as forças de superfície em forças nodais equivalentes. 
O desenvolvimento mostrado será feito para o carregamento transversal ao elemento triangular, e contudo o procedimento é estendido para as outras duas direções.

As forças de superfície transversais e as cargas nodais equivalentes são mostradas na figura 4.4 .

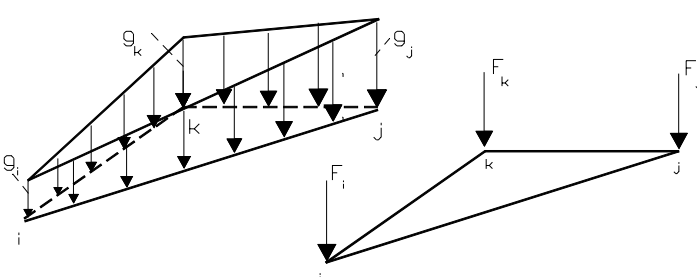

Figura 4.4 - Forças de superfície e cargas nodais equivalentes de um elemento.

O trabalho das cargas externas pode ser expresso por:

$$
T_{e}=\int_{A} g\left(x_{1}, x_{2}\right) \cdot w\left(x_{1}, x_{2}\right) d A
$$

onde $w\left(x_{1}, \mathrm{x}_{2}\right)$ são os deslocamentos transversais no domínio do elemento e A é a sua área. Para o caso em que este campo possui variação linear (figura 4.5), tem-se:

$$
w=w_{i} \xi_{1}+w_{j} \xi_{2}+w_{k} \xi_{3}
$$

Analogamente, as forças de superfície podem ser expressas por:

$$
g=g_{i} \xi_{1}+g_{j} \xi_{2}+g_{k} \xi_{3}
$$

Figura 4.5 - Variação linear do deslocamento transversal e da força de superfície no interior do elemento finito.

Transformando-se as coordenadas dos eixos cartesianos para as coordenadas homogêneas, e substituindo-se as expressões (4.50), (4.51) em (4.49) obtém-se: 


$$
T_{e}=\int_{A}\left(g_{i} \xi_{1}+g_{j} \xi_{2}+g_{k} \xi_{3}\right) \cdot\left(w_{i} \xi_{1}+w_{j} \xi_{2}+w_{k} \xi_{3}\right) d A
$$

Minimizando-se a energia potencial devida às cargas externas e sabendo-se que a integral $\int_{A} f\left(\xi_{1}, \xi_{2}, \xi_{3}\right) d A$ pode ser calculada como:

$$
\int_{A} \xi_{1}^{\eta_{1}} \xi_{2}^{\eta_{2}} \xi_{3}^{\eta_{3}} d A=2 A \frac{\eta_{1} ! \eta_{2} ! \eta_{3} !}{\left(\eta_{1}+\eta_{2}+\eta_{3}+2\right) !}
$$

chega-se ao vetor de cargas nodais transversais dado por:

$$
\left\{\begin{array}{l}
F_{i} \\
F_{j} \\
F_{k}
\end{array}\right\}=\frac{A}{12} \cdot\left[\begin{array}{lll}
2 & 1 & 1 \\
1 & 2 & 1 \\
1 & 1 & 2
\end{array}\right] \cdot\left\{\begin{array}{l}
g_{i} \\
g_{j} \\
g_{k}
\end{array}\right\}
$$

Seguindo-se o mesmo procedimento para as outras direções, a relação entre forças nodais e forças de superfície para o caso do elemento laminar DKT- Formulação Livre pode ser escrita como

$$
\left\{\begin{array}{l}
F_{i}^{\ell} \\
F_{j}^{\ell} \\
F_{k}^{\ell}
\end{array}\right\}=[\bar{Q}] \cdot\left\{\begin{array}{l}
g_{i}^{\ell} \\
g_{j}^{\ell} \\
g_{k}^{\ell}
\end{array}\right\}
$$

para $\mid=1,2,3$, representando as três direções e $i, j$ e $k$ os três nós locais do elemento, e a matriz $\bar{Q}$ dada por:

$$
[\bar{Q}]=\frac{A}{12} \cdot\left[\begin{array}{lll}
2 & 1 & 1 \\
1 & 2 & 1 \\
1 & 1 & 2
\end{array}\right]
$$


a)

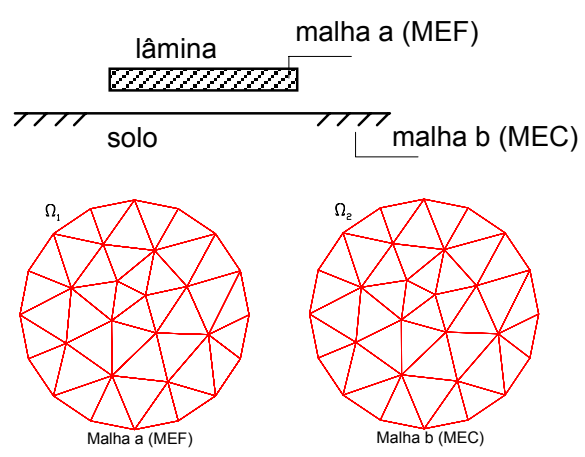

b)

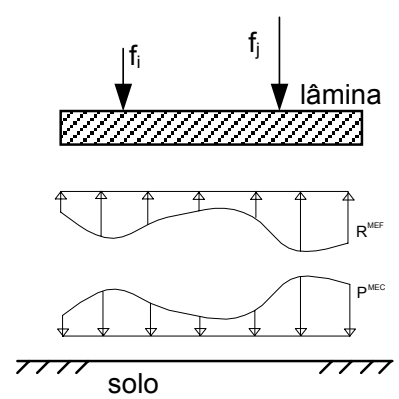

Figura 4.6 - a) Rede empregada para o solo e a lâmina em contato; b) forças de superfície e cargas nodais equivalentes.

$\mathrm{Na}$ expressão (4.19) podem-se incorporar as forças reativas da seguinte maneira:

$$
\left[K_{m e f}\right] \cdot\left\{U_{m e f}\right\}=\left\{F_{m e f}\right\}-\cdot\left\{R_{m e f}\right\}
$$

onde o vetor $R$ representa as forças concentradas de reação.

Assim, o acoplamento entre as forças de superfície advindas do MEC (equação 3.60), e as forças concentradas reativas advindas do MEF (relação $4.57)$, pode ser feito com o uso da matriz da relação (4.55), chegando-se a:

$$
\left[K_{\text {mef }}\right] \cdot\left\{U_{\text {mef }}\right\}=\left\{F_{\text {mef }}\right\}-[\overline{\bar{Q}}] \cdot[\hat{K}] \cdot\left\{U_{\text {solo }}\right\}
$$

onde $K_{\text {mef }}, U_{\text {mef }}$ e $F_{\text {mef }}$ são, respectivamente, a matriz de rigidez, os vetores dos parâmetros nodais de deslocamentos e forças concentradas da estrutura discretizada pelo MEF; $\overline{\bar{Q}}$ e $U_{\text {solo }}$ são, respectivamente, a matriz de transformação expandida relativa à contribuição de todos os elementos, expansão da relação (4.56), e o vetor de deslocamentos nodais da rede discretizada pelo MEC.

É possível reagrupar a relação (4.58), chegando-se a:

$$
K_{t o t} \cdot U_{t o t}=F_{t o t}
$$


Com

$$
K_{\text {tot }}=K_{m e f}+\overline{\bar{Q}} \cdot K_{m e c}
$$

E $U_{\text {tot }}$ e $F_{\text {tot }}$ são os deslocamentos e as forças totais do sistema soloestrutura. Esta matriz é não-simétrica e altamente esparsa, já que as influências entre os graus de liberdade dos dois modelos só ocorrem na região de contato, não havendo ligação entre as estruturas.

\subsection{Exemplos numéricos}

São apresentados três exemplos de estruturas. No primeiro e no segundo, são avaliados os comportamentos individuais de cada efeito, e o terceiro é um exemplo clássico para avaliação do efeito conjunto membranaplaca.

\subsubsection{Placa quadrada simplesmente apoiada}

Para ilustrar a aplicação do código desenvolvido, será analisada uma placa quadrada sob a ação de força uniformemente distribuída em toda a sua superfície e apoiada em todo seu extremo. O esquema da placa e os parâmetros geométricos e físicos são apresentados na figura 4.7. Para avaliar a correta formulação da matriz de transformação, analisa-se este exemplo no plano $\mathrm{X}_{2} \mathrm{X}_{3}$. 

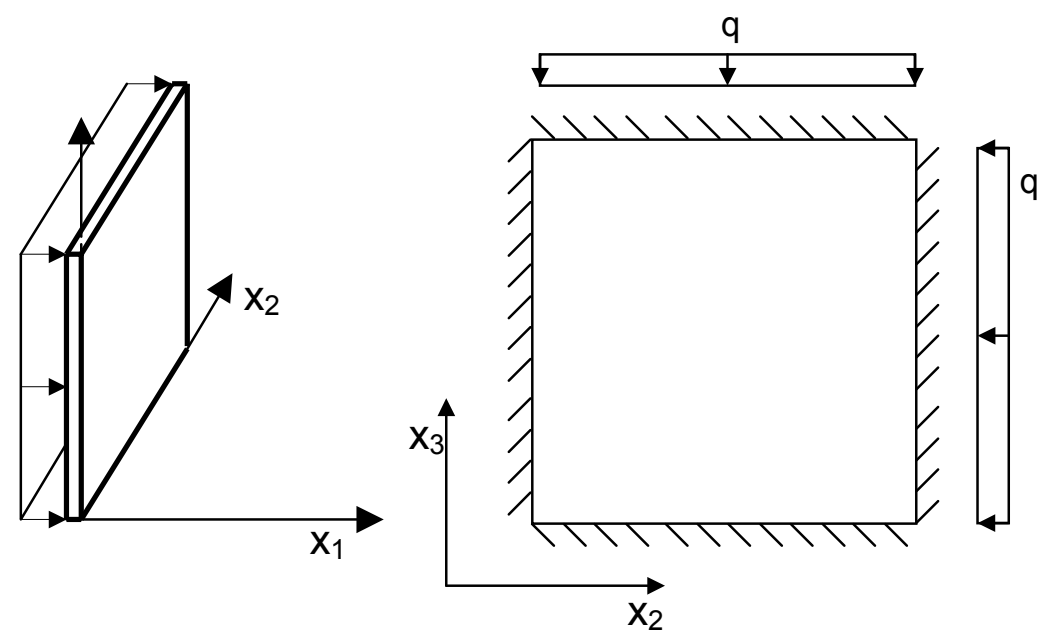

Valores utilizados
$E=10,92$
$t=1,0$ (espessura)
$q=1,0$
$L=1,0$
$v=0,3$

Figura 4.7 - Placa quadrada apoiada e parâmetros geométricos e físicos.

Tabela 4.1:

Deslocamentos, momentos e erros relativos no centro da placa para 3 tipos de redes.

\begin{tabular}{ccccc}
\hline $\mathrm{N}^{\circ}$ elementos & $\mathrm{u}_{\text {central }}$ & Erro (\%) & $\mathrm{M}_{\mathbf{x}^{\prime}}=\mathrm{M}_{\mathbf{y}^{\prime}}$ & Erro(\%) \\
\hline 128 & 0,00402341 & $-0,95$ & 0,048446 & 1,16 \\
512 & 0,00405271 & $-0,23$ & 0,048028 & 0,29 \\
1152 & 0,00405807 & $-0,09$ & 0,047949 & 0,12 \\
\multicolumn{4}{r}{ Valores analíticos( $\left.{ }^{*}\right): \mathbf{u}_{\text {central }}=\mathbf{0 , 0 0 4 0 6 2}$} & $\mathbf{M}_{\mathbf{x}^{\prime}}=\mathbf{M}_{\mathbf{y}^{\prime}}=\mathbf{0 , 0 4 7 8 9}$ \\
& $\left(^{*}\right)$ TIMOSHENKO \& WOINOWSKI-KRIEGER (1959) \\
\hline
\end{tabular}

Os valores encontrados de deslocamentos e momentos para o centro da placa mostraram boa concordância com os obtidos pela teoria analítica, indicando que uma maior discretização leva a um erro menor. Em relação às variáveis que representam os campos de membrana, os valores obtidos são praticamente nulos, como já era esperado. Não se utilizou a bi-simetria existente no problema, e caso fosse usada, para esta mesma densidade de elementos a convergência para a resposta exata seria mais acentuada.

\subsubsection{Cilindro com paredes rígidas}

Este é um clássico exemplo para se avaliar o efeito combinado de membrana e de flexão. Esta estrutura é um cilindro com as extremidades 
rígidas e com a aplicação de duas forças unitárias concentradas aplicadas em sentidos opostos da seção central do cilindro. Entretanto, por facilidade de geração de dados, discretiza-se apenas um oitavo, corrigindo essa simplificação através da imposição de restrições adequadas nos contornos da estrutura. Na figura 4.8, mostram-se o cilindro já simetrizado, o ponto de aplicação da força, as discretizações utilizadas, os parâmetros físicos e geométricos e a localização dos contornos para a avaliação dos resultados.

Para este exemplo, os parâmetros a serem calibrados na formulação livre foram adotados - conforme indicação de MESQUITA (1998) - como sendo $\alpha=1,2$ e $\beta=0,001$.

a) 128 elementos

D

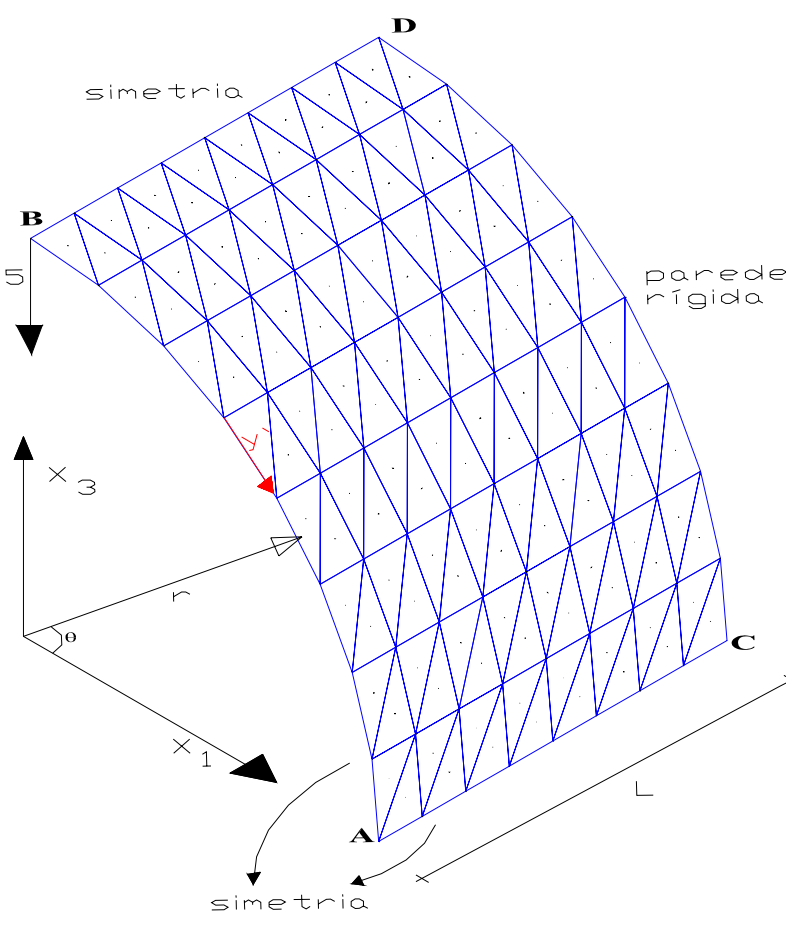

b) 574 elementos

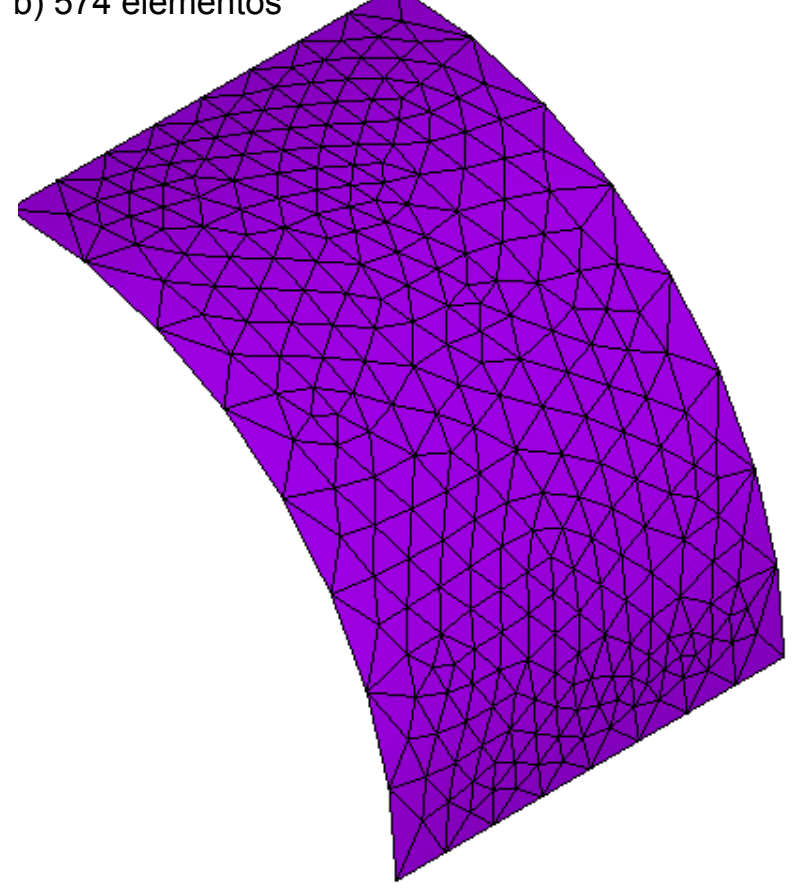

Figura 4.8 - Geometria e redes adotadas, sendo $E=300, v=0,3, L=R=300, h=3,0$

(espessura). 


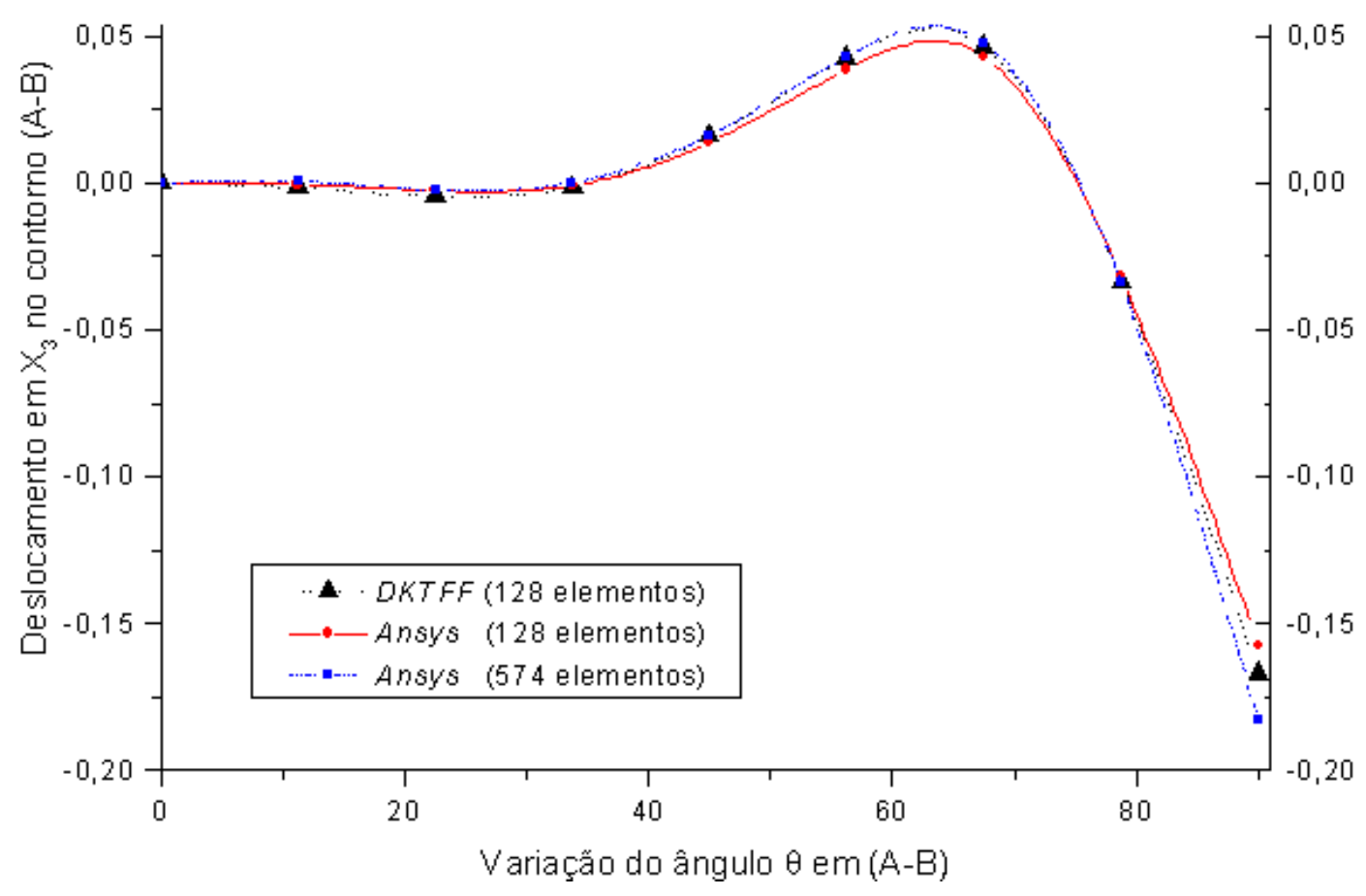

Figura 4.9 - Deslocamentos em $\mathrm{X}_{3}$ ao longo do contorno (A-B).

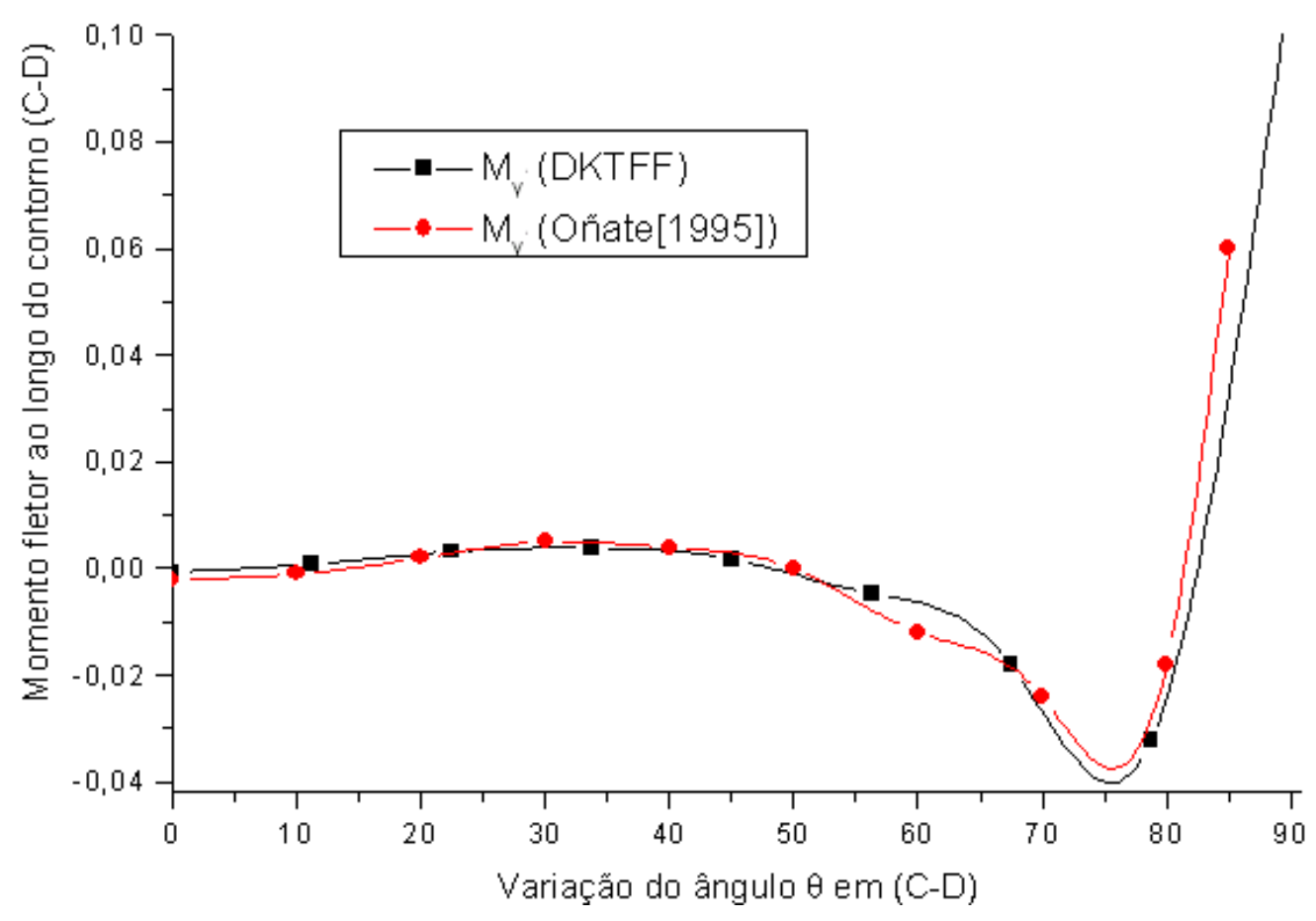

Figura 4.10 - Valores de momentos fletores na direção y' ao longo do contorno (C-D).

O elemento laminar do programa comercial Ansys utilizado foi o SHELL63, que possui quatro nós, cada um com 6 graus de liberdade. Assim, 
foi avaliado mediante o código computacional utilizando-se o elemento DKTFF, e comparados os resultados tanto com o programa do Ansys, como também com os valores apresentados em OÑATE (1995). Neste exemplo, utilizou-se apenas uma rede para o código com 128 elementos, sendo sua disposição esquematizada na figura 4.8 , onde as duas redes adotadas pelo programa Ansys são as apresentadas também nesta figura.

A figura 4.9 apresenta os resultados de deslocamentos ao longo do contorno (A-B). Em OÑATE (1995), comenta-se que a resposta exata para o deslocamento sob a força é de $(-0,18248)$, sendo que os erros relativos para - respectivamente- a rede do Ansys com 574 e 128 elementos e a rede utilizando o elemento DKTFF com também 128 foram de 0,17\%, 13,75\% e $8,55 \%$. Os dois últimos resultados de erros mostram que a formulação utilizando o elemento DKTFF leva a uma convergência mais rápida do que quando se utiliza o elemento SHELL63 do Ansys.

Com relação aos momentos fletores em coordenadas locais, a figura 4.10 compara os valores da rede utilizando o elemento DKTFF com os apresentados por OÑATE (1995). Nota-se que os valores mostrados por este autor são obtidos mediante uma refinada discretização - 1600 elementos quadrangulares. Como esses momentos para a curva do DKTFF e de OÑATE (1995) são obtidos em sistemas locais diferentes, este gráfico tem mais o intuito de mostrar semelhanças qualitativas. Pode-se notar uma boa proximidade entre as duas curvas. 


\section{Capítulo 5}

\section{EXEMPLOS NUMÉRICOS}

\subsection{Introdução}

Uma formulação numérica deve ser avaliada e principalmente confirmada mediante a simulação de exemplos. Pois, transferir o modelo numérico para um código computacional envolve a possibilidade da geração de mais erros somados aos já consagrados erros de máquina. Assim, é imprescindível para a confirmação da formulação apresentar exemplos que simulem as diferentes situações que a formulação deva avaliar. Neste sentido, esse capítulo apresenta alguns exemplos que demonstram a eficiência, a potencialidade, e até as correções que devam ser feitas dentro do código ou da formulação propriamente dita.

A formulação apresentada nos capítulos anteriores foi implementada em um código computacional usando-se o compilador Fortran do pacote da Digital Visual Fortran versão 6.0 para ambiente Windows. Foram empregadas rotinas internas desse pacote denominado de IMSL (do inglês Internal Mathematics and Statistics Library), desenvolvido por Aird et al. (1977), e também rotinas otimizadas do pacote BLAS (Basic Linear Algebra Subprograms), que tem referência o trabalho de LAWSON et al. (1979).

Os exemplos, em sua grande maioria, foram extraídos de outros trabalhos. As redes dos elementos empregados para a modelagem foram geradas da seguinte maneira: a) a geração dos elementos de estaca são feitas automaticamente, mediante o emprego de um gerador desenvolvido pelo autor; 
b) para a construção das superfícies que representam o solo e os elementos laminares foram empregados ou o programa de geração de malhas criado por SOUSA JR. (1996), denominado de PEC, ou pelo emprego do gerador contido no pacote comercial do Ansys 5.4 ; c) a geração do edifício foi feita mediante o emprego de um gerador também desenvolvido pelo autor. Destaca-se que o gerador do programa comercial Ansys 5.4 não realiza - explicitamente para o usuário - uma otimização procurando o menor comprimento de banda, sendo, portanto, inadequado seu uso como gerador para outros programas de elementos finitos que não realizem essa otimização sobre os dados gerados ou que realizem a montagem convencional da matriz de rigidez.

É necessário salientar que a presente formulação é mais geral do que os modelos empregados nos trabalhos de comparação aqui utilizados, pois todos eles não computam, no sistema algébrico final, as equações referentes aos campos de deslocamentos horizontais ( $u$ e $v$ ) para o solo, uma vez que suas formulações de placa não contemplam estes efeitos.

\subsection{Lâmina quadrada apoiada sobre meio semi-infinito}

Este exemplo foi apresentado em PAIVA (1993) que analisa a interação entre a placa e o solo semi-infinito. $O$ intuito de se colocar este exemplo é para poder avaliar o comportamento da presente formulação para a simulação o problema clássico de uma placa apoiada sobre o solo homogêneo em meio infinito.

Assim, para simular o meio semi-infinito, adotou-se uma camada de espessura de $2000 \mathrm{~m}$ para o maciço (figura 5.1a). A rede de elementos de contorno empregada na interface entre a lâmina/solo está indicada na figura 5.1b, e seus parâmetros físicos e geométricos são apresentados na figura 5.1c.

Avaliou-se a interação lâmina/solo com dois tipos de valores rigidez da lâmina, denominadas de lâmina intermediária e rígida (vide figura 5.1c), e compararam-se seus resultados de deslocamentos e de tensões de contato nas 
regiões de interface entre a lâmina e o solo. Foram empregadas duas redes de elementos de contorno para representar a região do solo, de modo que cada rede foi criada levando-se em conta a relação entre o diâmetro da região discretizada do solo e o comprimento da lâmina. Assim, as duas redes apresentam as relações de $D_{\text {solo }} / D_{\text {lâmina }}=10$ e $D_{\text {solo }} / D_{\text {lâmina }}=50$ (vide figura $5.2 a \mathrm{e}$ $5.2 b)$.

Para o caso da lâmina rígida, aplicou-se uma força concentrada no seu centro geométrico. Os resultados de deslocamentos e tensões verticais são apresentados na tabela 5.1, e demonstram uma boa concordância com os obtidos por outras formulações. PAIVA (1993) formula o problema de modo que o solo e a placa delgada são representados por equações integrais com o uso do MEC, onde o solo é modelado pela solução de Mindlin. MESSAFER \& COATES (1989) e MENDONÇA (1997) empregam o MEC via solução de Mindlin para representar o meio semi-infinito, e para a placa delgada ambos empregam o MEF. Entretanto, MESSAFER \& COATES (1989) dividem a placa em 100 elementos finitos quadrangulares do tipo ACM, enquanto MENDONÇA (1997) usa os elementos finitos triangulares DKT e HSM. GORBUNOVPOSSADOV \& SEREBRJANY (1961) obtêm os resultados para este problema analiticamente.

Pelos resultados apresentados na tabela 5.1 , as menores diferenças de deslocamentos ocorreram entre a presente formulação, o trabalho analítico de GORBUNOV-POSSADOV \& SEREBRJANY (1961) e MESSAFER \& COATES (1989), onde a diferença é, respectivamente, de $-2,54 \%$ e $5,58 \%$. Já para os resultados de tensões de contato, as diferenças de valores com esses trabalhos foram as maiores quando comparadas com as outras duas formulações: PAIVA (1993) e MENDONÇA (1997). É importante salientar que, conforme a tabela 5.1 , os resultados obtidos pela presente modelagem para as duas redes se diferenciam em torno de $2,50 \%$ para os deslocamentos, e $4,25 \%$ para as tensões de contato. 
Para o caso da lâmina ter rigidez intermediária, aplicou-se uma força distribuída uniformemente constante sobre sua área, e a figura 5.3 apresenta os resultados de deslocamentos no eixo de referência " $S$ " para as duas redes empregadas neste exemplo, comparando-os com os obtidos por PAIVA (1993) e MESSAFER \& COATES (1989). A diferença relativa do deslocamento central da lâmina entre a presente formulação, usando a rede 2 , e os valores apresentados pelos dois citados, são de $16,04 \%$ para o trabalho de PAIVA (1993), e de 4,25\% para MESSAFER \& COATES (1989). Salienta-se que essa diferença relativa entre os dois últimos trabalhos é de $11,33 \%$.

A figura 5.4 mostra os resultados de tensões verticais da região de contato entre a lâmina e o solo no eixo "S". Nota-se a boa concordância entre os resultados entre as diferentes formulações, e conforme se aproxima do centro da lâmina, as diferenças vão se diminuindo.

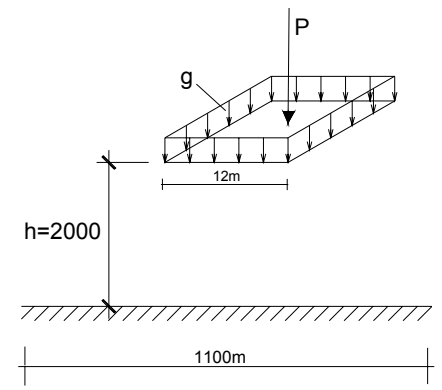

a)

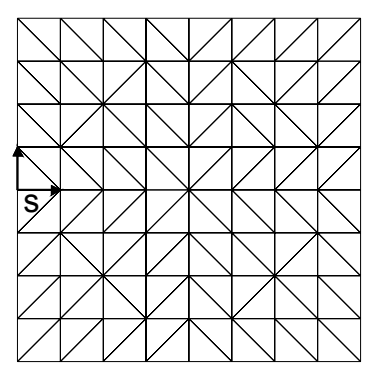

b)

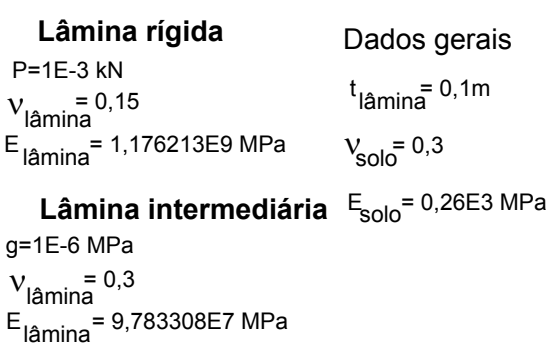

c)

Figura 5.1 - a) esquema estrutural do modelo; b) rede usada para discretizar a lâmina; c) parâmetros físicos e geométricos do conjunto lâmina/solo. 


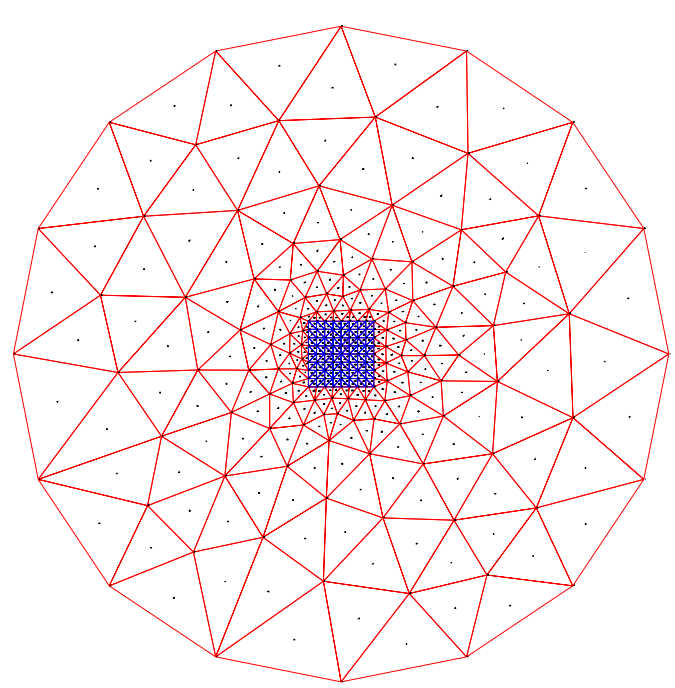

a) $D_{\text {solo }} / D_{\text {lâmina }}=10$

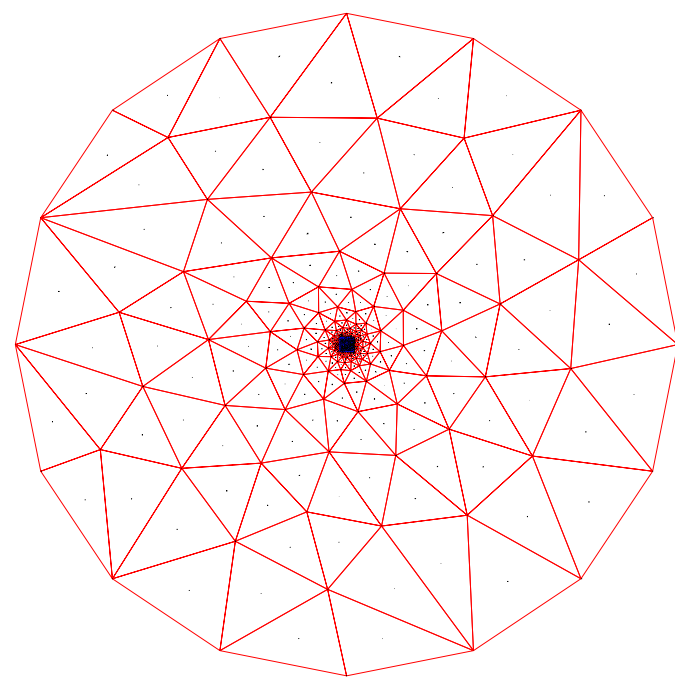

b) $D_{\text {solo }} / D_{\text {lâmina }}=50$

Figura 5.2 - Rede usada para discretizar a superfície livre do solo. a) Rede 1;b) Rede 2.

i) Lâmina rígida

Tabela 5.1:

Valores de deslocamentos e forças de contato verticais para o centro da lâmina.

\begin{tabular}{ccc}
\hline Referência & $\boldsymbol{W} * \mathbf{1 0}^{\mathbf{9}}(\boldsymbol{m})$ & Tensões de contato $\left(\mathbf{N} / \mathbf{m}^{\mathbf{2}}\right)$ \\
\hline Paiva (1993) - MEC-MEC & 0,2160 & 0,0045 \\
Messafer \& Coates (1989) & 0,2400 & 0,0038 \\
Gorbunov- Possadov \& & 0,2600 & 0,0040 \\
Serebrjany (1961) & & 0,0042 \\
Mendonça (1997)- HSM-MEC & 0,2122 & 0,0042 \\
Mendonça (1997)- DKT-MEC & 0,2124 & 0,0047 \\
Presente Trabalho - Rede 1 & 0,2599 & 0,0045 \\
Presente Trabalho - Rede 2 & 0,2534 & \\
\hline
\end{tabular}




\section{ii) Lâmina intermediária}

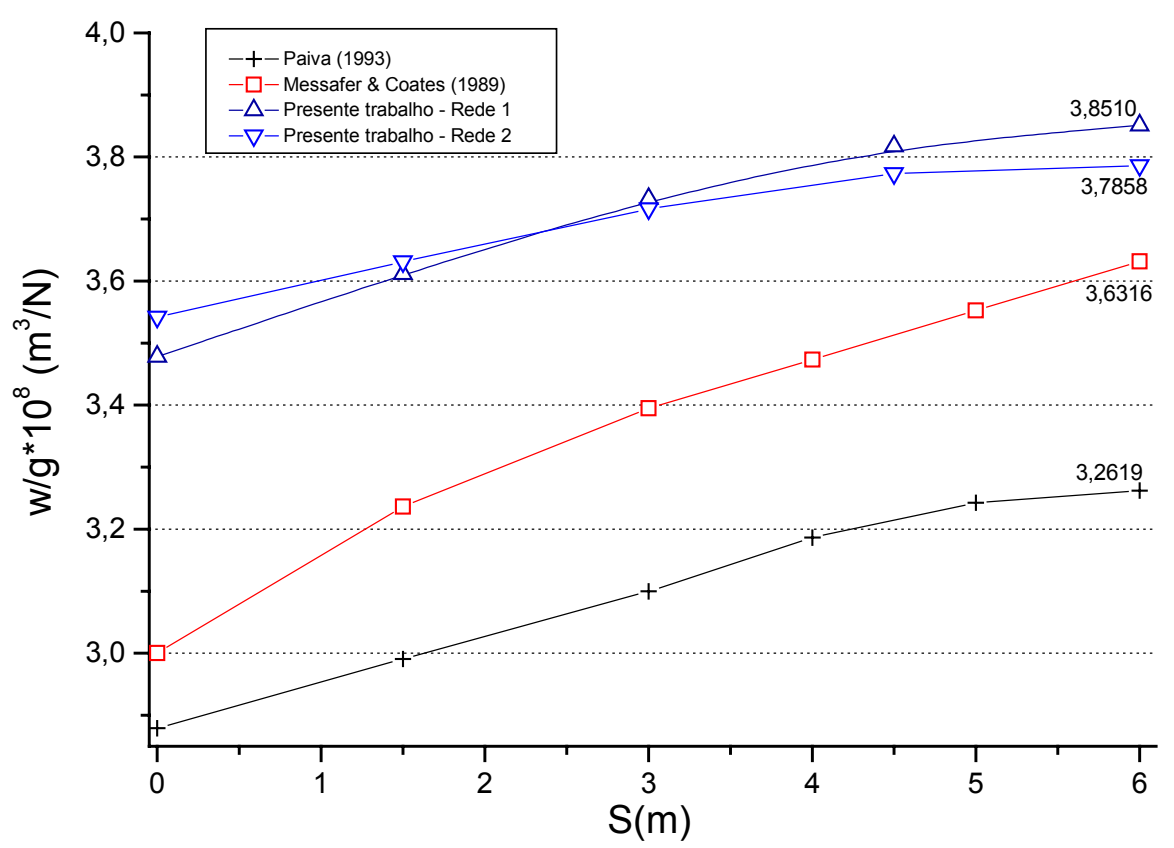

Figura 5.3 - Deslocamentos verticais da superfície do maciço sobre o eixo S.

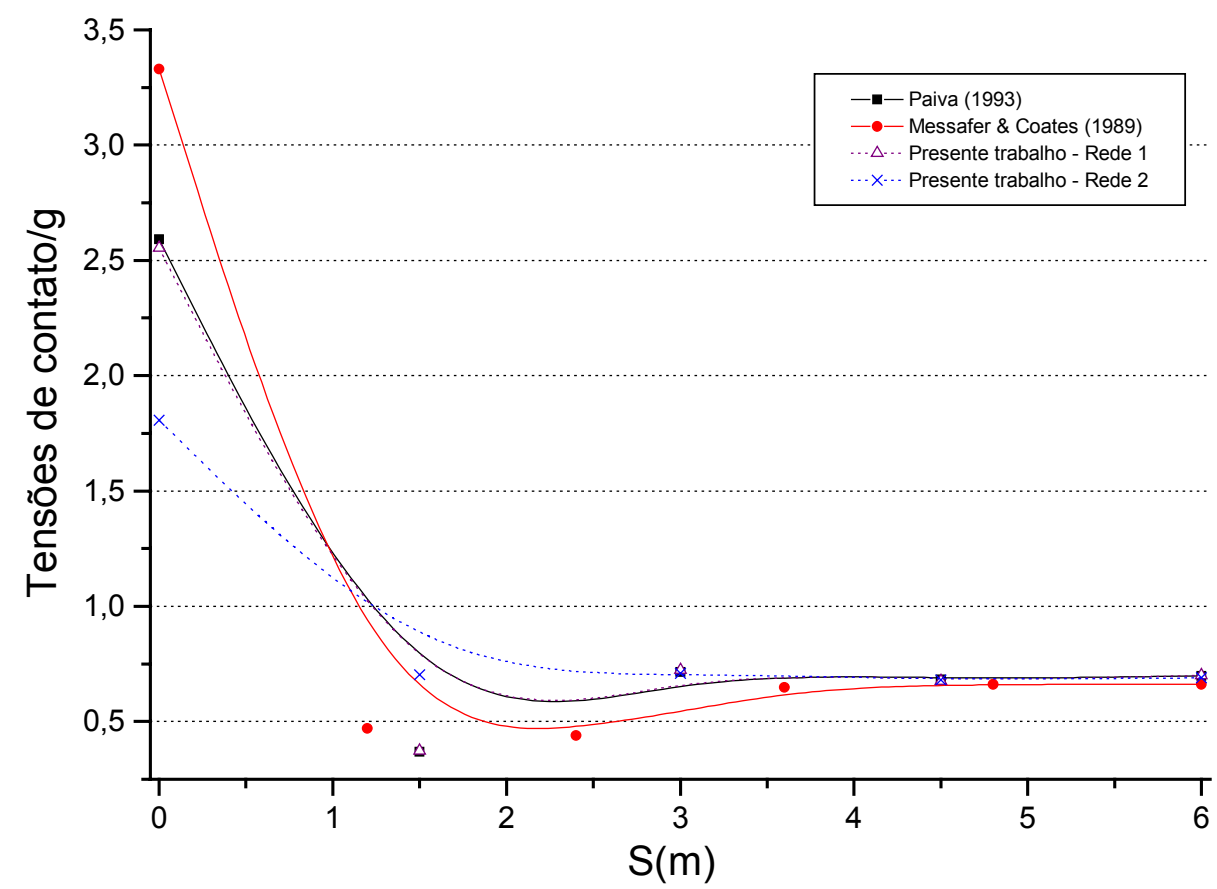

Figura 5.4 - Forças de superfície do contato lâmina/solo sobre o eixo S. 


\subsection{Camada finita com variação linear do módulo de elasticidade}

Apresenta-se neste item uma análise do solo não-homogêneo, linear e isótropo, onde o intuito deste exemplo é mostrar o comportamento da formulação perante a consideração de várias sub-regiões que formarão os diversos estratos do solo com suas respectivas propriedades físicas para simular o efeito de rigidez crescente linearmente, sem considerar as estacas e/ou superestruturas.

Considera-se, então, a ação de forças distribuídas uniformemente de valor constante em uma região circular da superfície livre do solo. A lei que governa o módulo de elasticidade transversal é uma função linear crescente (veja figura 5.5i), e a simulação dessa variação da rigidez do solo é feita empregando várias sub-regiões, denominadas de camadas, com espessura constante, e para cada uma se atribui um módulo de rigidez constante com seu valor obtido na sua cota média.

Os resultados apresentados pela tabela 5.2 mostram que para uma espessura total pequena do solo - fator de espessura $h / a=2$ - a consideração de poucas camadas do solo, simulando o efeito crescente da rigidez, leva rapidamente a resultados bem próximos aos obtidos pela semi-analítica. Já para o caso de uma espessura maior - $h / a=8$ - é necessário se empregar muitas camadas para simular o efeito da variação crescente da rigidez.

As diferenças relativas dos deslocamentos centrais da região carregada são obtidas comparando-se os valores encontrados por esta formulação e os calculados pelas expressões semi-analíticas tiradas de BURMISTER (1945). 


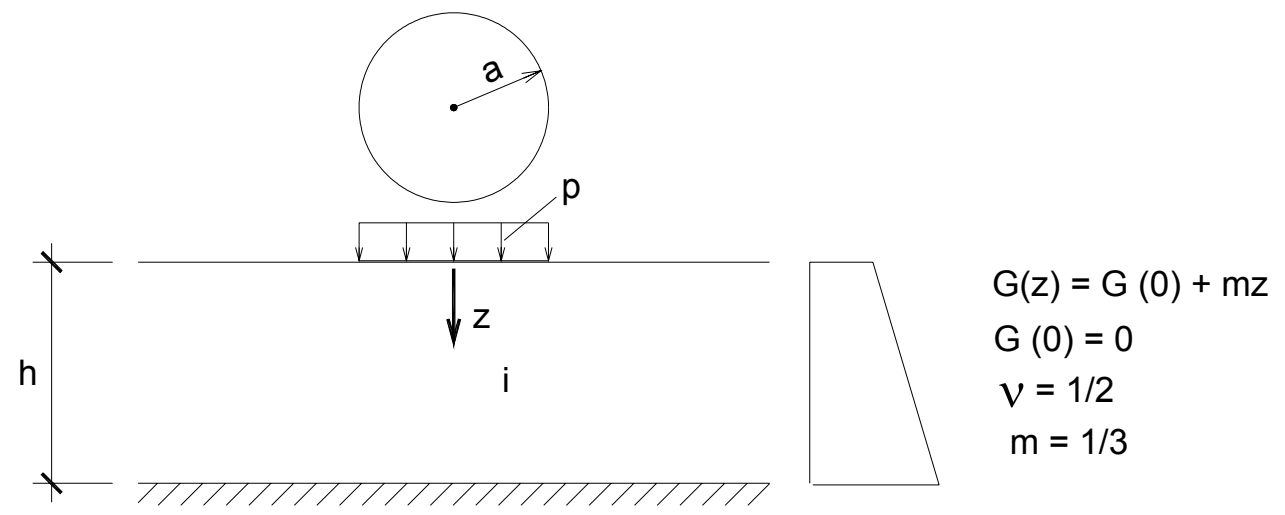

i)

ii)

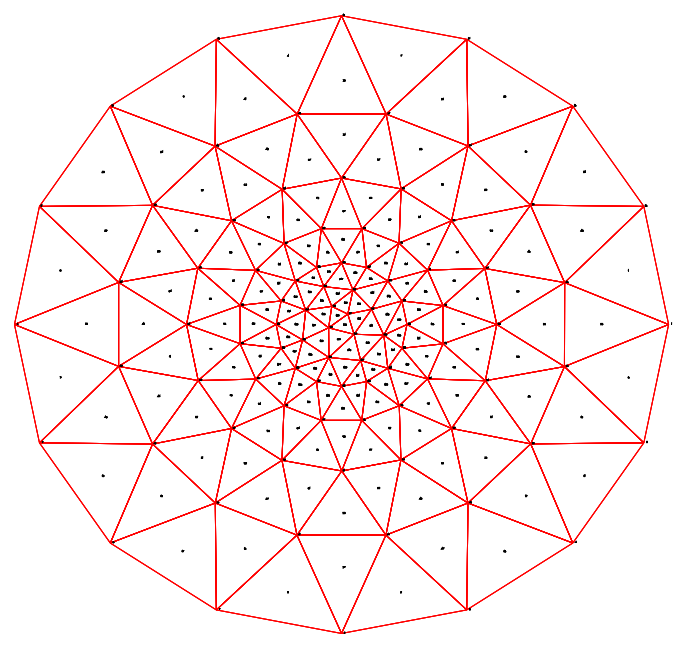

Figura 5.5 - i) estrato finito sob carregamento aplicado em área circular ii) rede usada para o solo.

Tabela 5.2:

Erro porcentual dos deslocamentos centrais da superfície.

\begin{tabular}{ccc}
\hline Fator de espessura h/a & Camadas & Erro (\%) \\
\hline 2 & 2 & 13,36 \\
2 & 3 & 2,20 \\
2 & 5 & 1,54 \\
\hline 8 & 3 & 52,96 \\
8 & 7 & 19,44 \\
8 & 20 & 0,71 \\
\hline
\end{tabular}




\subsection{Lâmina fina sobre uma base não-deformável}

Este problema tem o intuito de avaliar as respostas de deslocamentos e de momentos fletores que se mobilizam quando do carregamento aplicado sobre uma lâmina quadrada em contato com o solo, no qual a base de seu indeslocável está a uma profundidade de $10 \mathrm{~m}$ da superfície. A figura 5.6 ilustra a discretização usada no modelo da lâmina, as características físicas e geométricas do solo homogêneo.

Os resultados da presente formulação são comparados com outros trabalhos. Verifica-se uma grande concordância com os valores obtidos por RASER \& WARDLE (1974) e SADECKA (2000). Os primeiros autores modelam o espaço semi-infinito usando a técnica dos elementos de superfície (ver WARDLE \& FRASER (1974)), onde a matriz final é obtida usando-se as técnicas de transformações de integral. O segundo trabalho avalia os deslocamentos ao longo da profundidade do meio por meio de funções pesos não lineares, e admite-se a influência da lâmina apoiada na superfície do solo usando o MEF. 


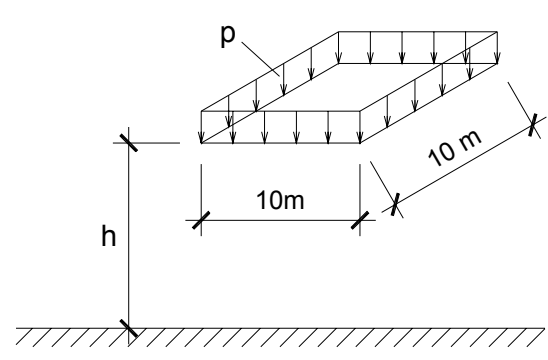

a)

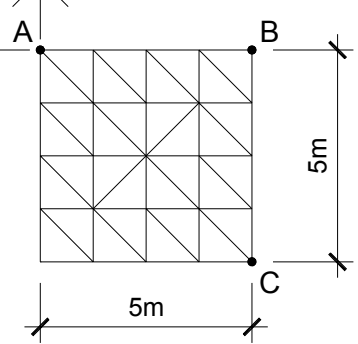

b)

$$
\begin{aligned}
& \mathrm{p}=0,01 \mathrm{MPa} \\
& \mathrm{E}_{\text {solo }}=9,1 \mathrm{MPa} \\
& \mathrm{v}_{\text {solo }}=0,3 \\
& \mathrm{E}_{\text {lâmina }}=21000 \mathrm{MPa} \\
& \mathrm{v}_{\text {lâmina }}=0,15 \\
& \mathrm{t}_{\text {lâmina }}=0,26 \mathrm{~m} \\
& \mathrm{~h}=10 \mathrm{~m}
\end{aligned}
$$

c)

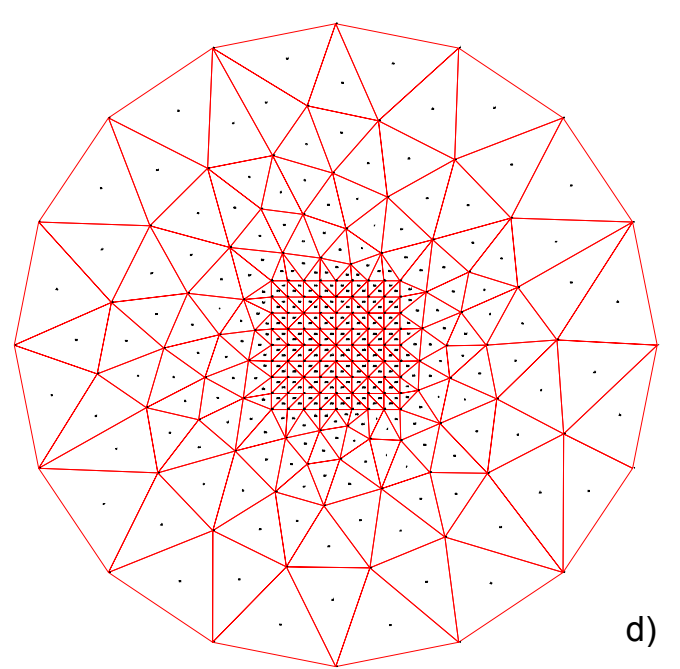

Figura 5.6 - a) esquema da lâmina sobre meio indeformável; b) rede de um quarto da lâmina; c) parâmetros físicos e geométricos do solo e da lâmina; d) rede usada para o solo.

Tabela 5.3:

Resultados de deslocamentos e momentos fletores para pontos da lâmina.

\begin{tabular}{ccccc}
\hline Pontos da lâmina & $\begin{array}{c}\text { Fraser \& Wardle } \\
(1974)\end{array}$ & $\begin{array}{c}\text { Kolar̂ \& Nemec } \\
(1983)\end{array}$ & $\begin{array}{c}\text { Sadecka } \\
(2000)\end{array}$ & $\begin{array}{c}\text { Presente } \\
\text { trabalho }\end{array}$ \\
\hline A [Desloc. $\left.\mathrm{w}^{*} 10^{-3}(\mathrm{~m})\right]$ & 7,30 & 5,36 & 6,18 & 6,47 \\
B [Desloc. $\left.\mathrm{w}^{*} 10^{-3}(\mathrm{~m})\right]$ & 4,50 & 4,73 & 3,97 & 4,62 \\
C [Desloc. $\left.\mathrm{w}^{*} 10^{-3}(\mathrm{~m})\right]$ & 2,80 & 3,76 & 2,25 & 2,95 \\
\hline A [Momento $\left.\mathrm{M}_{11}\left(\mathrm{kN} / \mathrm{m}^{2}\right)\right]$ & 6,20 & 3,09 & 6,58 & 6,22 \\
\hline
\end{tabular}




\subsection{Lâmina quadrada sobre um meio homogêneo de base rígida}

Neste exemplo, a superfície livre do estrato está sujeita a um carregamento unitário distribuído uniformemente em uma região quadrada (ver figura 5.7). Os resultados de deslocamentos para o presente método são comparados com os obtidos com o método aproximado de Steinbrenner e com o modelo de BURMISTER (1945).

Os valores resultantes são listados na tabela 5.4 para vários níveis de profundidades. Para a construção da rede discretizada, foram empregados 748 nós dos quais 441 nós e 800 elementos estão localizados na região central de aplicação da carga, e os restantes 307 nós e 518 elementos estão representando a região não-carregada (figura $5.7 \mathrm{~b}$ ). O módulo de elasticidade adotado do solo tem valor de $100 \mathrm{MPa}$, e seu índice de Poisson é de 0,3.

A tabela 5.4 mostra boa concordância entre os valores obtidos por esta formulação e o modelo semi-analítico de BURMISTER (1945). Conforme indicado por POULOS (1967), o método aproximado de Steinbrenner subestima os valores de deslocamentos, e à medida que o plano indeslocável se aproxima da superfície livre do solo, os valores são de $10 \%$ a $15 \%$ menores. Por outro lado, a presente formulação demonstra que esta variação da cota do indeslocável não influencia nos resultados, mantendo o erro sempre abaixo de $1 \%$. 

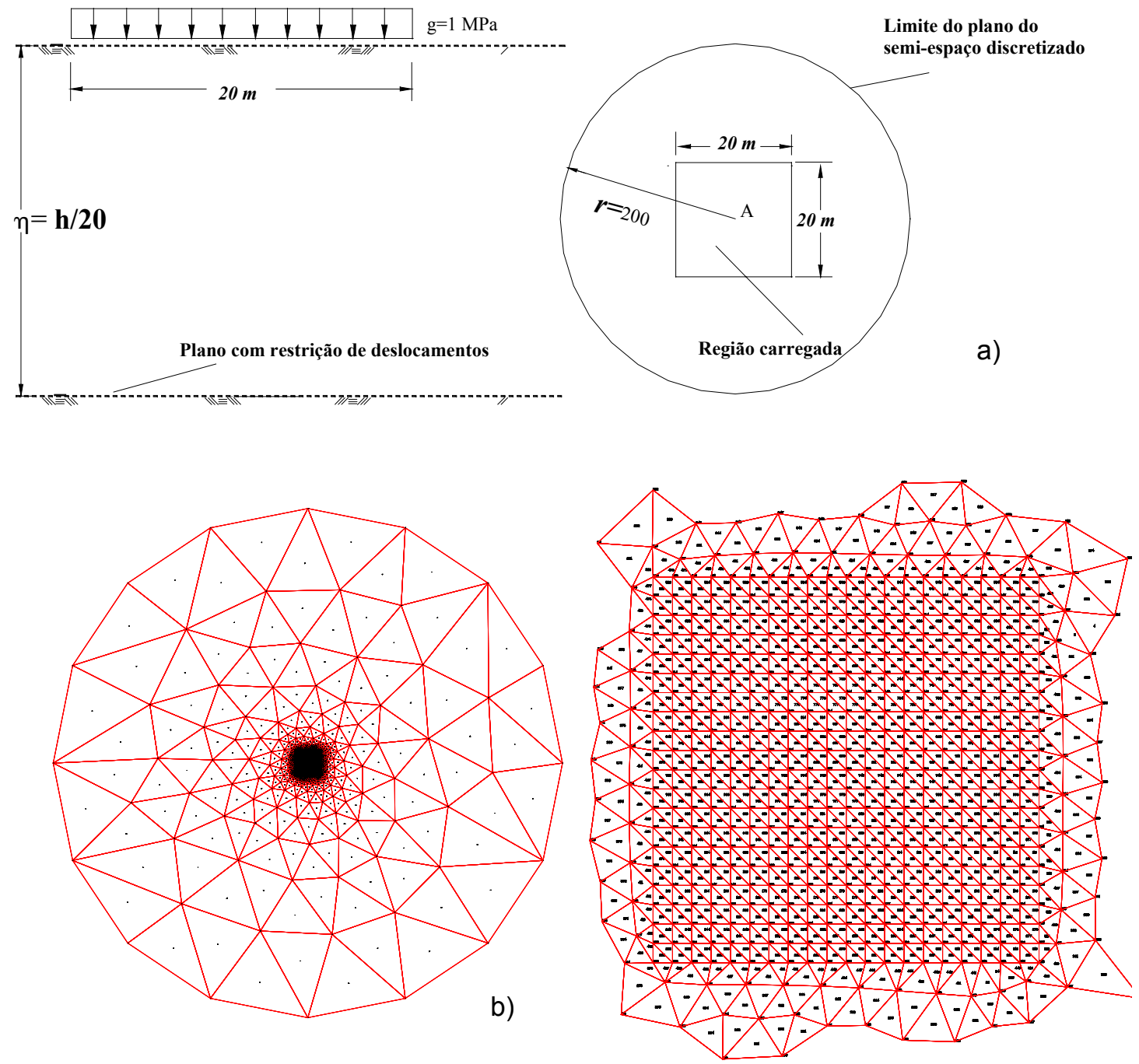

Figura 5.7 - a) estrato finito sob carregamento uniforme; b) rede usada para todo o solo (esquerda) e para a região de contato entre solo/lâmina (direita).

Tabela 5.4:

Deslocamento vertical $(\mathrm{m})$ do ponto A.

\begin{tabular}{cccc}
\hline$\eta=h / 20$ & $\begin{array}{c}\text { Burmister } \\
\text { (1945) }\end{array}$ & $\begin{array}{c}\text { Presente trabalho } \\
\text { (\% erro) }\end{array}$ & $\begin{array}{c}\text { Steinbrenner } \\
\text { (\% erro) }\end{array}$ \\
1 & 0,1290 & $0,1299(0,7)$ & $0,1171(9,2)$ \\
2 & 0,1639 & $0,1650(0,7)$ & $0,1564(4,6)$ \\
5 & 0,1876 & $0,1883(0,4)$ & $0,1845(1,7)$ \\
50 & 0,2026 & $0,2037(0,5)$ & $0,2022(0,2)$ \\
\hline
\end{tabular}




\subsection{Edifício sobre radier apoiado em um maciço não-homogêneo}

Este exemplo tem a finalidade de averiguar o efeito da variação tanto das propriedades do solo ao longo da profundidade quanto da posição da base do indeslocável causa sobre um edifício de múltiplos andares apoiado sobre um radier. As redes de elementos utilizadas para discretizar o solo e a lâmina são idênticas ao exemplo anterior (ver figura 5.7a e 5.7b), uma vez que os erros relativos obtidos para a simulação do maciço foram de $0,7 \%$ e $0,5 \%$ para, respectivamente, as relações de $\eta=1$ e $\eta=50$.

Mediante a planta de um pavimento-tipo de 12 andares para fins residenciais, as ações permanentes foram calculadas conforme a norma brasileira NBR 6118 (2000). As ações variáveis de vento consideradas conforme a NBR 6123 (1987), onde o edifício foi suposto localizado em São Carlos, velocidade de vento $V_{0}=42 \mathrm{~m} / \mathrm{s}$, o valor topográfico $S_{1}$ foi considerado de 1,0 , pois o terreno foi considerado plano, com poucas ondulações, e ele foi incluído no grupo 2: edificações para hotéis e residências, com alto fator de ocupação. Para o fator estatístico $S_{2}$, o edifício foi enquadrado na classe $B$ e na categoria IV. Para o fator $S_{3}$ foi atribuído o valor de 1,0.

Obtiveram-se, então, as deformações e os esforços nas vigas, nos pilares e no radier, os deslocamentos e as forças de superfície (tensões de contato) entre o solo e o radier, e deslocamentos e tensões no domínio do maciço. Na figura 5.8, são apresentadas as plantas da estrutura analisada, as tabelas da geometria e das ações consideradas em cada elemento estrutural do pavimento tipo. O módulo de elasticidade adotado para o edifício tem valor de $2,5 \times 10^{4} \mathrm{MPa}$, e o coeficiente de Poisson para o solo é igual para todos os casos com valor de 0,3 . Para o radier adotou-se o mesmo módulo de elasticidade do edifício, o coeficiente de Poisson de 0,2 , a sua espessura de $0,5 \mathrm{~m}$, mas não se considerou o seu peso próprio. Os parâmetros da formulação livre usados foram de $\alpha=1,5$ e $\beta=0,01$. 


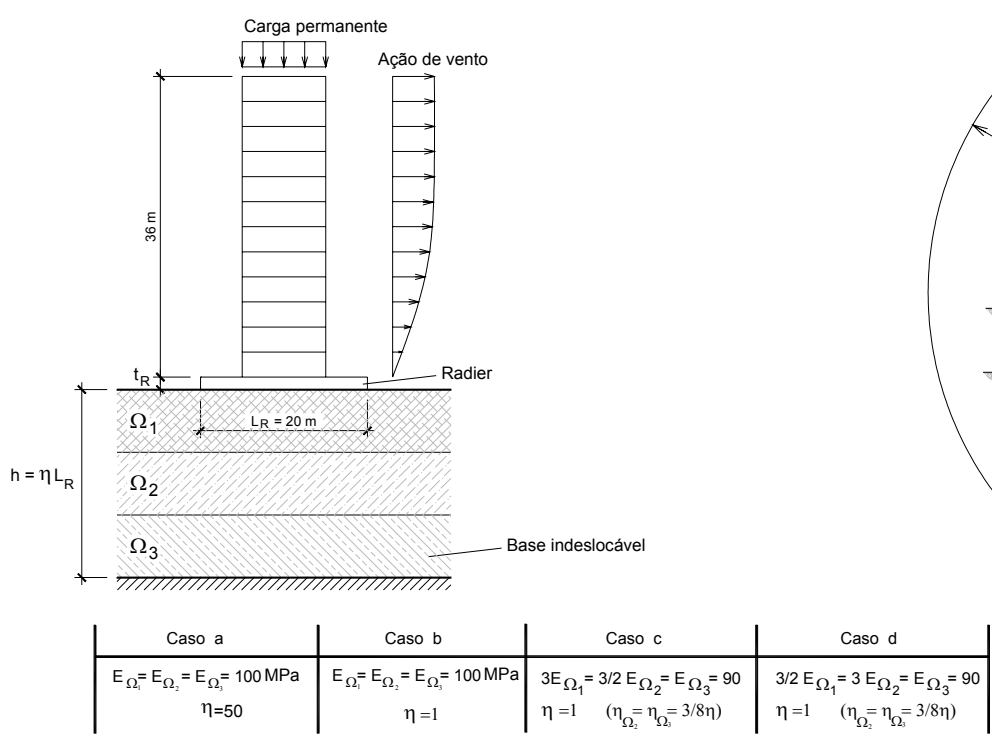

a)

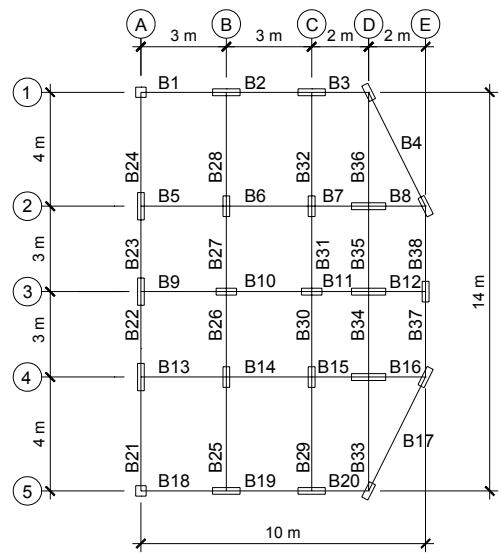

c)

\begin{tabular}{|c|c|c|}
\hline \multicolumn{3}{|c|}{ Carga permanente $(\mathrm{kN} / \mathrm{m})$} \\
\hline Andar tipo & Cobertura & Vigas \\
\hline 4,5 & 3,0 & $\mathrm{~B} 1, \mathrm{~B} 18, \mathrm{~B} 22, \mathrm{~B} 23$ \\
\hline 3,75 & 2,5 & $\mathrm{~B} 2, \mathrm{~B} 19$ \\
\hline 3,0 & 2,0 & $\mathrm{~B} 3, \mathrm{~B} 20$ \\
\hline 0,67 & 0,45 & B4,B17 \\
\hline 9,0 & 6,0 & $\begin{array}{l}\mathrm{B} 5, \mathrm{~B} 7, \mathrm{~B} 9, \mathrm{~B} 13, \mathrm{~B} 15 \\
\mathrm{~B} 26, \mathrm{~B} 27, \mathrm{~B} 30, \mathrm{~B} 31\end{array}$ \\
\hline 9,75 & 6,5 & $\mathrm{~B} 6, \mathrm{~B} 14, \mathrm{~B} 29, \mathrm{~B} 32$ \\
\hline 8,5 & 5,67 & B34,B35 \\
\hline 6,0 & 4,0 & $\mathrm{~B} 8, \mathrm{~B} 12, \mathrm{~B} 16$ \\
\hline 12,0 & 8,0 & $\frac{\mathrm{B} 10, \mathrm{~B} 11}{1}$ \\
\hline 5,63 & 3,75 & B21,B24 \\
\hline 10,78 & 7,12 & $\mathrm{~B} 25, \mathrm{~B} 28$ \\
\hline 8,25 & 5,5 & $\mathrm{~B} 33, \mathrm{~B} 36$ \\
\hline 4,0 & 2,67 & $\mathrm{~B} 37, \mathrm{~B} 38$ \\
\hline TIPO & ILARES $\left(\mathrm{m}^{2}\right)$ & VIGAS $\left(\mathbf{m}^{2}\right)$ \\
\hline 1 & $0,4 \times 0,4$ & $0,2 \times 0,4$ \\
\hline 2 & $0,2 \times 0,6$ & $0,2 \times 0,3$ \\
\hline 3 & $0,2 \times 0,8$ & - \\
\hline 4 & $0,2 \times 1,2$ & - \\
\hline 5 & $0,2 \times 0,4$ & - \\
\hline
\end{tabular}

d)

Figura 5.8 - a) corte da estrutura e os casos considerados; b) planta do modelo edifício/radier; c) planta baixa do edifício; d) tabelas dos parâmetros dos elementos de barra do edifício. 
A figura 5.9 apresenta os resultados de deslocamentos transversais $(w)$ ao longo do eixo $x_{1}$ do radier, corte AA da figura $5.8 \mathrm{~b}$, já considerando a interação solo/radier/edifício para os quatro tipos de casos indicados na figura 5.8a. Como era de se esperar, os deslocamentos mobilizados pela configuração do caso $b$ deram menores. Na figura 5.10 é mostrada a distribuição de deslocamentos ao longo do radier para o caso $b$, e se pode evidenciar o seu comportamento radialmente simétrico, derivado pela redistribuição feita pelo radier das ações do edifício sobre o solo, e esse mesmo comportamento é verificado para os demais casos.

As figuras 5.11 e 5.12 apresentam os diagramas de momentos fletores $M_{11}$ e $M_{22}$ de todo o radier para o caso $b$, onde é possível se notar que os maiores valores recaem sobre os pontos onde os pilares estão conectados ao radier. O valor máximo dessas solicitações, caso se queira dimensionar o radier como uma laje de concreto armado convencional, leva ao domínio 2 com taxa de armadura de $0,33 \%$, onde a taxa mínima é de $0,10 \%$.

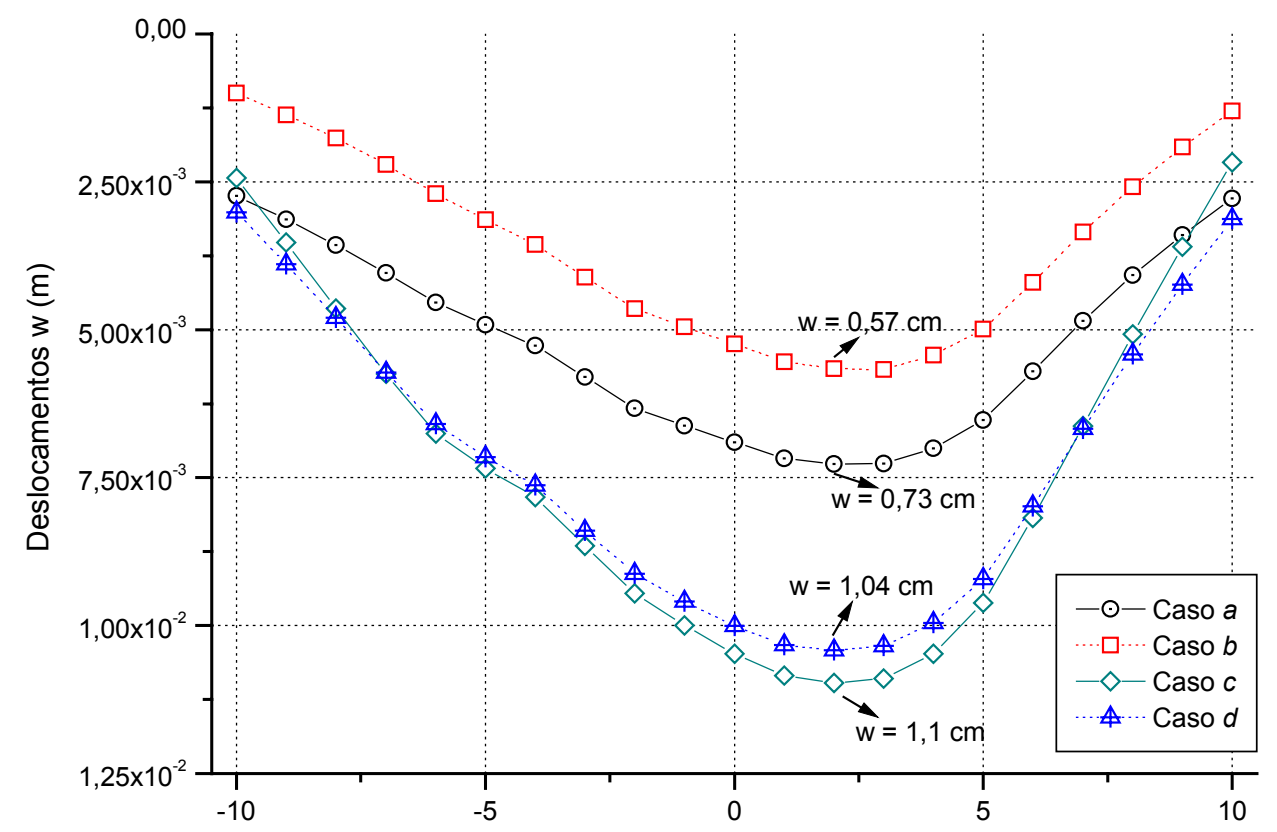

Figura 5.9 - Deslocamentos verticais ao longo do corte AA. 


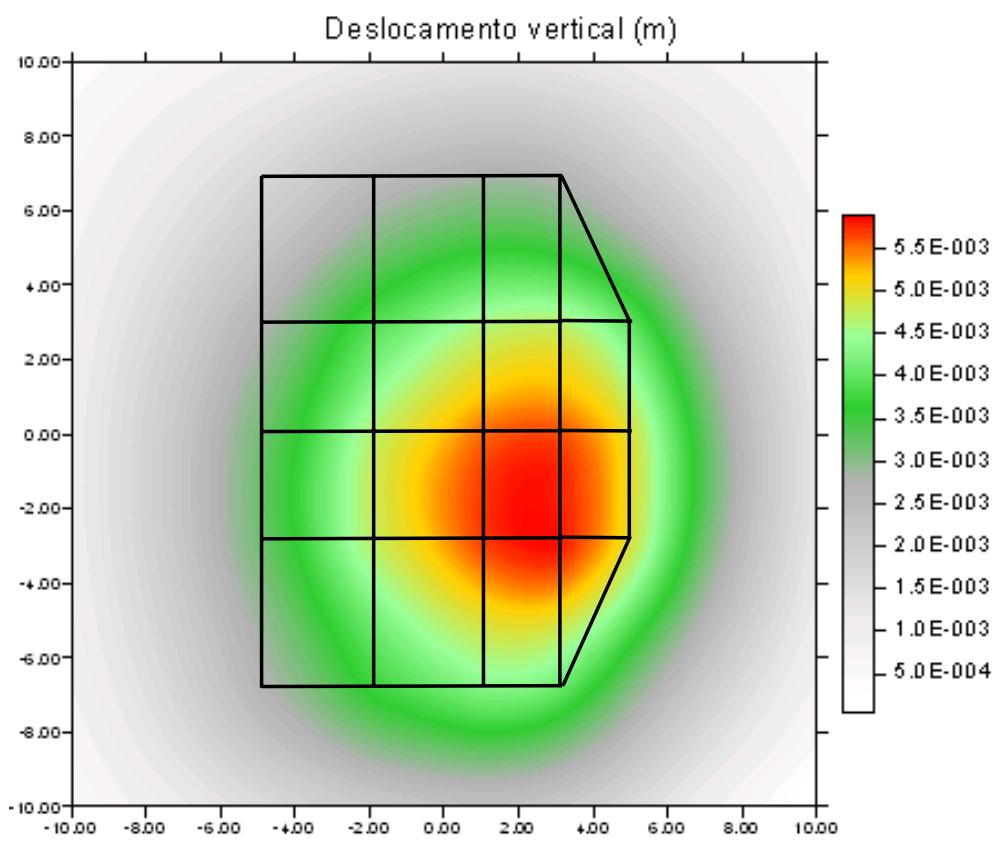

Figura 5.10 - Deslocamentos verticais no radier para o caso $b$.

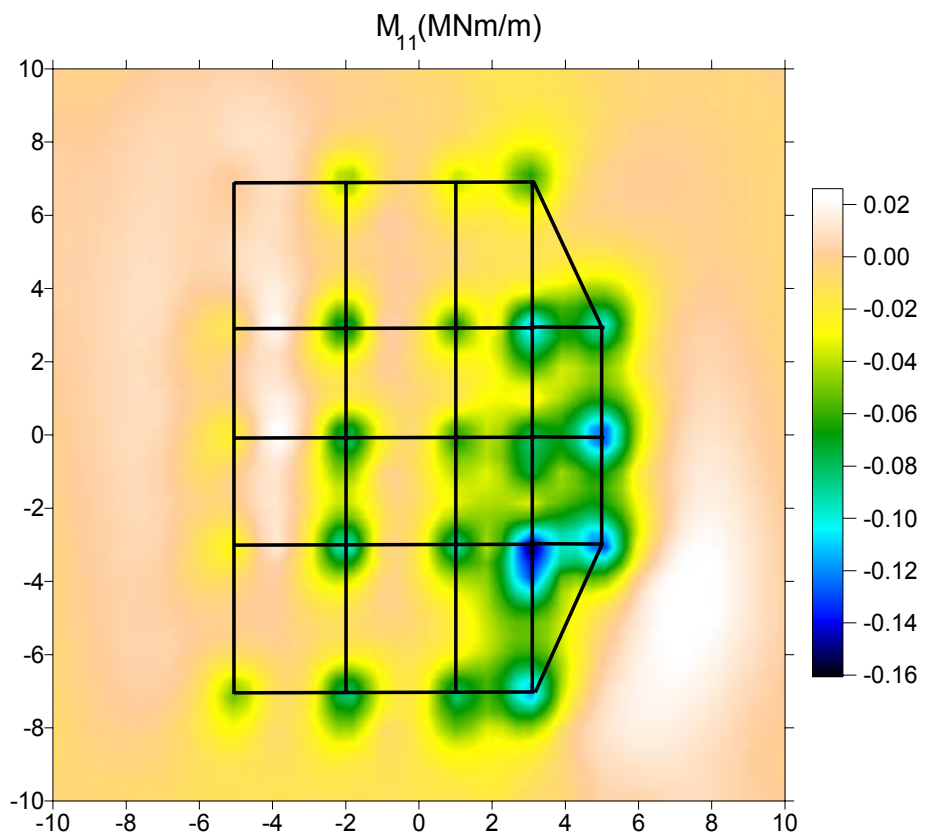

Figura 5.11 - Momentos fletores $M_{11}$ sobre todo o radier para o caso $b$. 


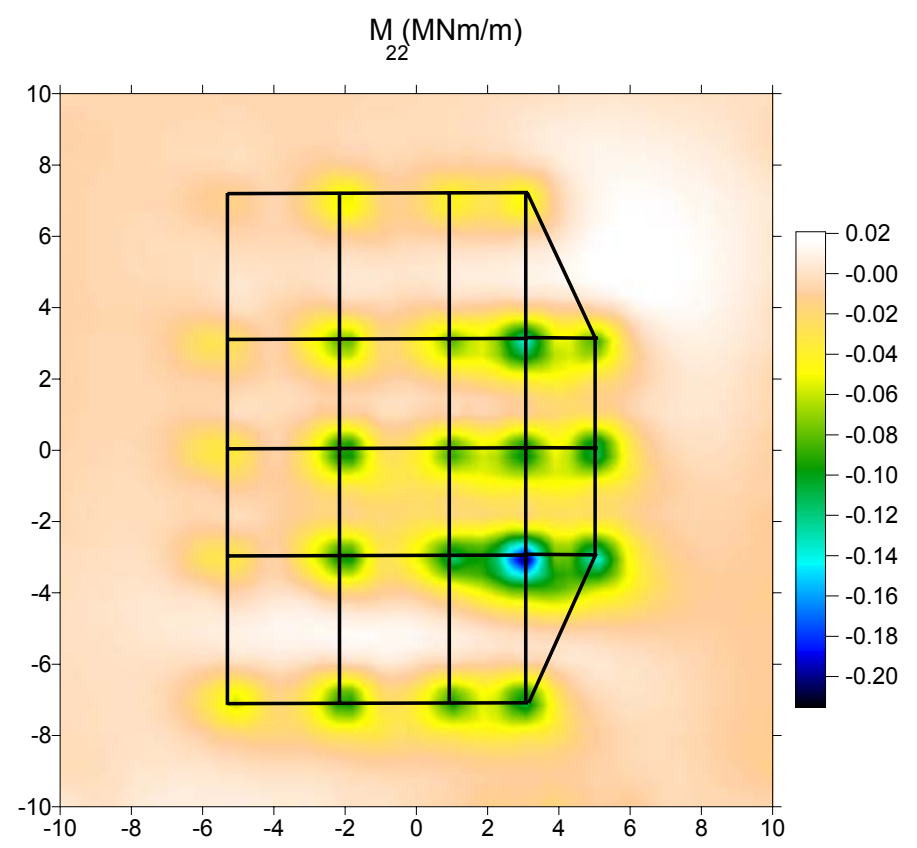

Figura 5.12 - Momentos fletores $M_{22}$ sobre todo o radier para o caso $b$.

A figura 5.13 apresenta a distribuição das tensões verticais de contato entre o radier e o solo, onde é possível se verificar que os valores são, em média, em torno de 0,06 MPa. Conforme as recomendações da norma de projeto e execução de fundações, NBR 6122 (1996), as pressões admissíveis para fundações superficiais dependem da classe em que o solo se enquadra. Para o caso do solo ser uma areia medianamente compacta, este valor é de 0,2 $M P a$, então se obtêm um coeficiente de segurança de 3,3 , onde a norma recomenda um valor nunca menor que 3 .

As figuras 5.14 e 5.15 apresentam, respectivamente, os valores de tensões e de deslocamentos na direção vertical do caso $c$ para o corte $\mathrm{BB} . \mathrm{Na}$ figura 5.14 é possível notar como a distribuição de tensões ocorre ao longo da espessura do meio, e para este caso onde o plano indeslocável está localizada a uma distância igual ao tamanho do radier, essas tensões não se anularam 
ainda no plano indeformável, podendo se prever que elas se anulem a distância de duas vezes o tamanho do radier.

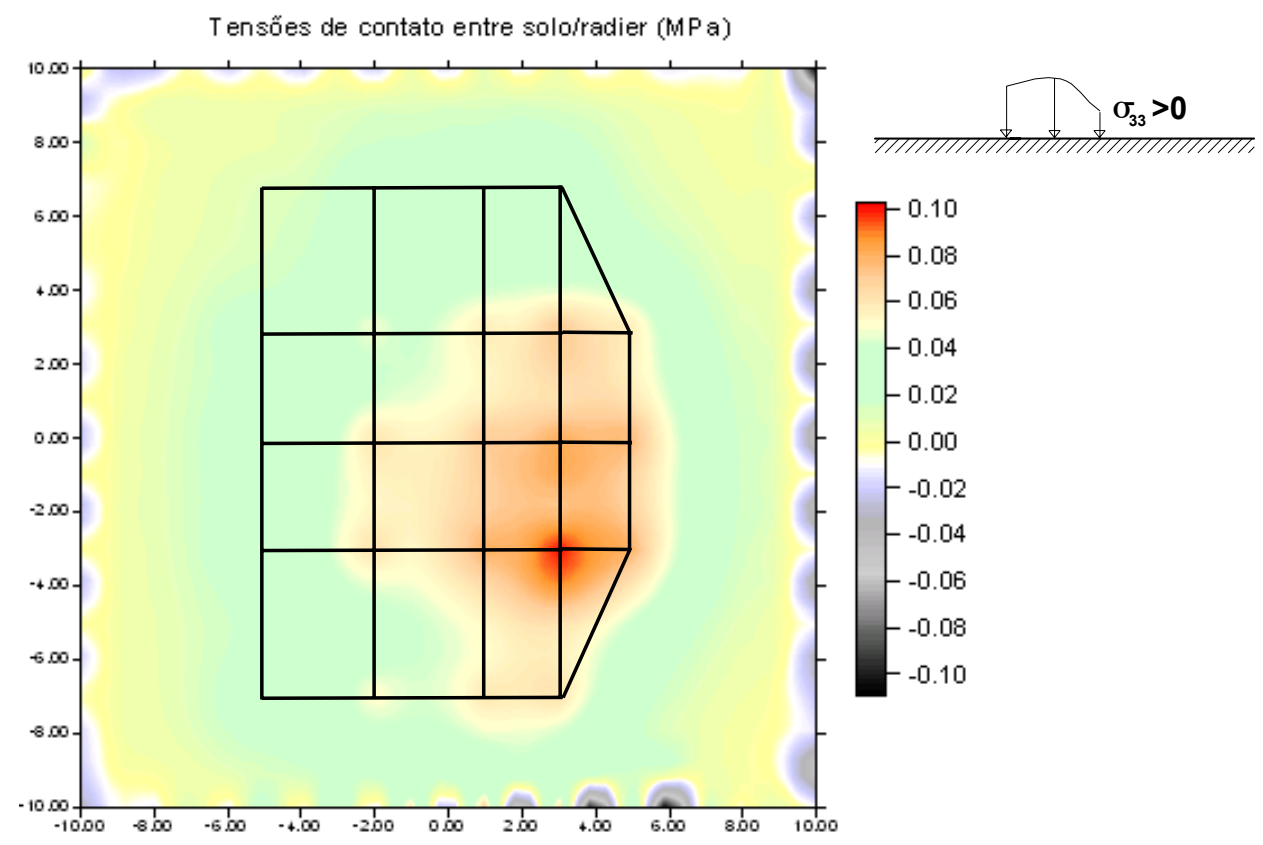

Figura 5.13 - Tensões de contato $\left(\sigma_{33}\right)$ na região comum ao radier e ao solo para o caso $b$.

Na tabela 5.5 são apresentados os valores das forças normais de todos os pilares do andar térreo do edifício, e na tabela 5.6 avaliam-se as porcentagens absolvidas pelos pilares de canto, de extremidade e intermediários dos cinco casos estudados. Quando a deformabilidade do solo é considerada, os pilares intermediários ficam menos sobrecarregados, assim há uma redistribuição de esforços mais uniforme, isto porque a mobilização do sistema solo/radier/edifício ocorre de maneira conjunta, o que difere da análise do edifício apoiado em radier indeformável quando não se considera esta deformabilidade do solo. Além disso, as variações dos valores das forças normais quando se considera ou não a deformabilidade do solo chegaram a diferenças de mais de $20 \%$ para alguns pilares. 


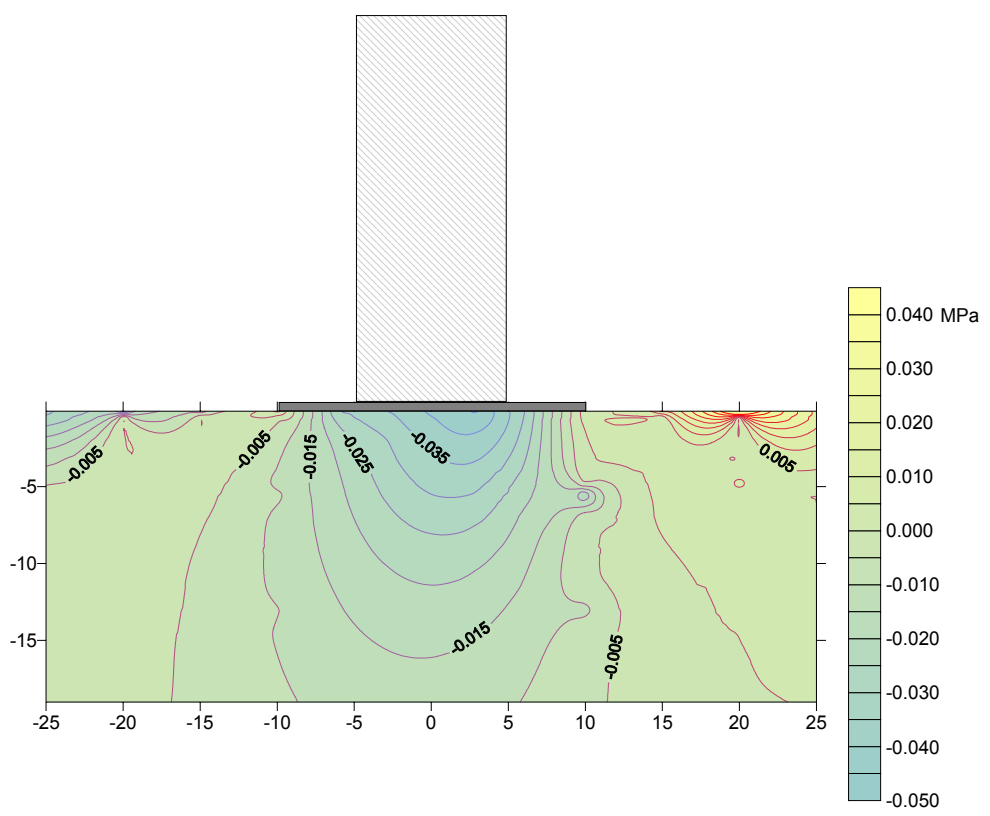

Figura 5.14 - Distribuição de tensões verticais $\left(\sigma_{33}\right)$ no solo ao longo do corte BB para o caso $c$.

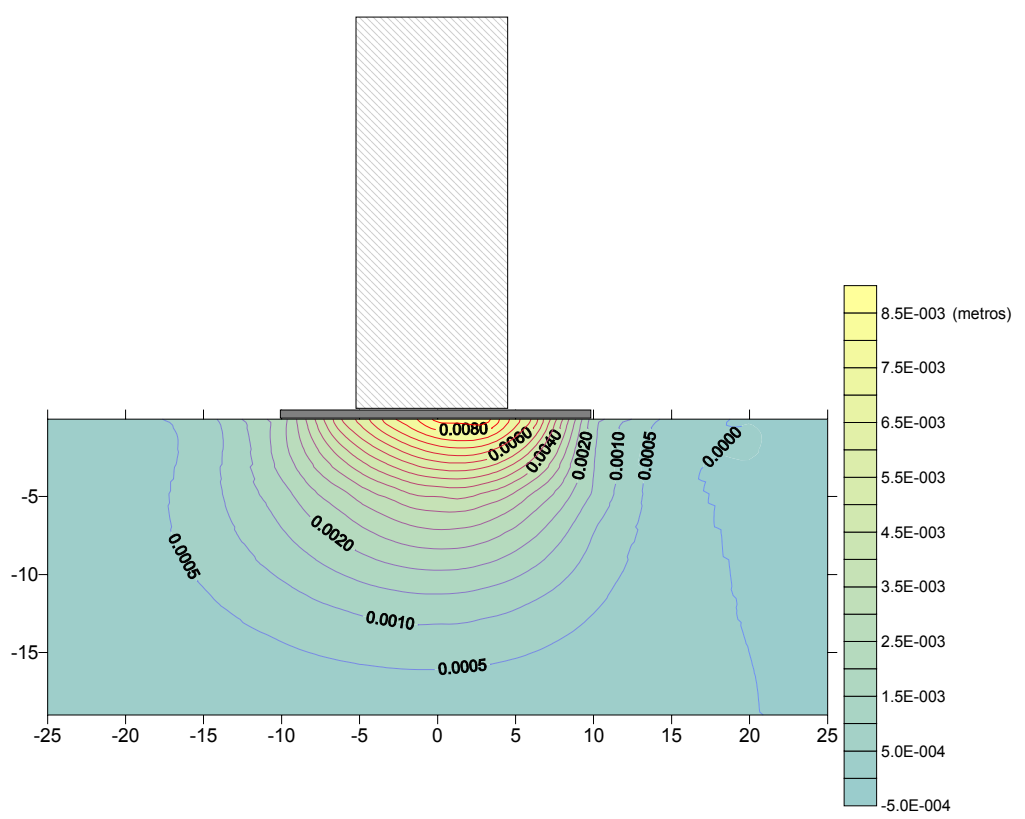

Figura 5.15 - Distribuição de deslocamentos $\left(\mathrm{u}_{3}\right)$ no solo ao longo do corte BB para o caso $c$. 
A figura 5.16 mostra os deslocamentos na direção $x_{1}$ e $x_{2}$ do nó mestre do edifício, ponto $A$, para os casos de base indeformada e para o caso $b$, mostrando que a consideração da compressibilidade do maciço leva a maiores deslocamentos deste e, assim, tornando-se a análise do sistema solo/radier/edifício também importante para a verificação do efeito de segunda ordem do edifício.

A figura 5.17 apresenta a distribuição de momentos fletores ao longo do pilar A3 para o segundo e terceiro andar, e para a viga B9. Mostra-se, então, esses valores para o caso em que não se considera a deformabilidade do solo e para o caso $c$, ou seja, a variação crescente da rigidez do maciço. Com isso, verifica-se que a influência da deformabilidade no sistema é importante, principalmente para os esforços mobilizados devido ao peso próprio, onde as diferenças relativas entre os casos foram de até $202 \%$. As diferenças dos esforços devido ao efeito de vento não ultrapassaram valores de $10 \%$ para estes elementos reticulares.

Tabela 5.5:

Forças normais (MN) nos pilares do andar térreo para os vários casos de rigidez do solo. Sinal positivo indica compressão nos pilares.

\begin{tabular}{|c|c|c|c|c|c|}
\hline Pilar & Caso a & Caso $\boldsymbol{b}$ & Caso $\mathbf{c}$ & Caso d & $\begin{array}{c}\text { Solo } \\
\text { indeformável }\end{array}$ \\
\hline A1 & 0,0687 & 0,0661 & 0,1139 & 0,0896 & 0,0359 \\
B1 & 0,3021 & 0,3024 & 0,3222 & 0,3694 & 0,2875 \\
C1 & 0,2172 & 0,2188 & 0,2314 & 0,1668 & 0,1997 \\
D1 & 0,3074 & 0,3103 & 0,3593 & 0,4183 & 0,2390 \\
\hline A2 & 0,1369 & 0,1402 & 0,1475 & 0,0563 & 0,1017 \\
B2 & 0,4670 & 0,4666 & 0,4330 & 0,4457 & 0,5153 \\
C2 & 0,3726 & 0,3709 & 0,3279 & 0,2990 & 0,4219 \\
D2 & 0,6253 & 0,6176 & 0,6090 & 0,6187 & 0,6756 \\
E2 & 0,4473 & 0,4512 & 0,517 & 0,5258 & 0,3363 \\
\hline A3 & 0,1886 & 0,1894 & 0,2021 & 0,2001 & 0,1509 \\
B3 & 0,5477 & 0,5449 & 0,5116 & 0,5142 & 0,6205 \\
C3 & 0,3983 & 0,3998 & 0,3301 & 0,3452 & 0,4821 \\
D3 & 0,4224 & 0,4257 & 0,3028 & 0,3410 & 0,5933 \\
E3 & 0,6225 & 0,6246 & 0,6732 & 0,6605 & 0,5366 \\
\hline
\end{tabular}




\begin{tabular}{|l|c|c|c|c|c|}
\hline A4 & 0,1911 & 0,1912 & 0,2034 & 0,2944 & 0,1366 \\
B4 & 0,5671 & 0,5669 & 0,5024 & 0,5072 & 0,6420 \\
C4 & 0,4744 & 0,4743 & 0,3827 & 0,4445 & 0,5762 \\
D4 & 0,8172 & 0,8127 & 0,7408 & 0,7661 & 0,9707 \\
E4 & 0,6223 & 0,6230 & 0,6917 & 0,6431 & 0,4993 \\
\hline A5 & 0,3087 & 0,303 & 0,3868 & 0,3816 & 0,2404 \\
B5 & 0,5094 & 0,5096 & 0,5364 & 0,4756 & 0,4790 \\
C5 & 0,4279 & 0,4309 & 0,4471 & 0,5023 & 0,3968 \\
D5 & 0,5658 & 0,5672 & 0,6355 & 0,5424 & 0,4703 \\
\hline
\end{tabular}

Tabela 5.6:

Porcentagem das forças normais distribuídas nos pilares de extremidade, intermediário e de canto.

\begin{tabular}{|c|c|c|c|c|c|}
\hline Tipo de pilar & Caso a & Caso b & Caso c & Caso d & $\begin{array}{c}\text { Solo } \\
\text { indeformável }\end{array}$ \\
\hline Canto & $13 \%$ & $13 \%$ & $16 \%$ & $15 \%$ & $10 \%$ \\
Extremidade & $38 \%$ & $38 \%$ & $41 \%$ & $40 \%$ & $33 \%$ \\
Intermediário & $49 \%$ & $49 \%$ & $43 \%$ & $45 \%$ & $57 \%$ \\
\hline
\end{tabular}

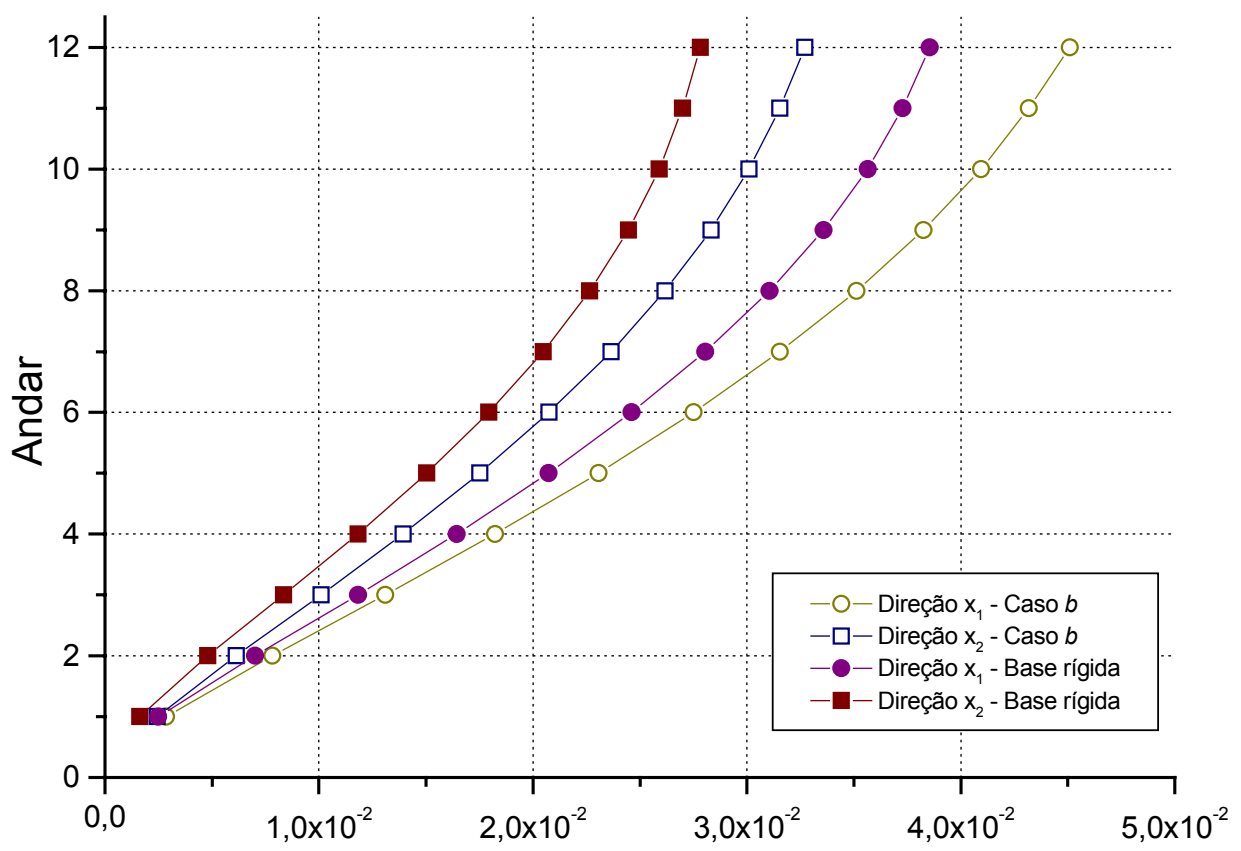

Figura 5.16 - Curva deslocamentos horizontais (metros) versus andares do nó mestre do edifício para o caso $b$. 

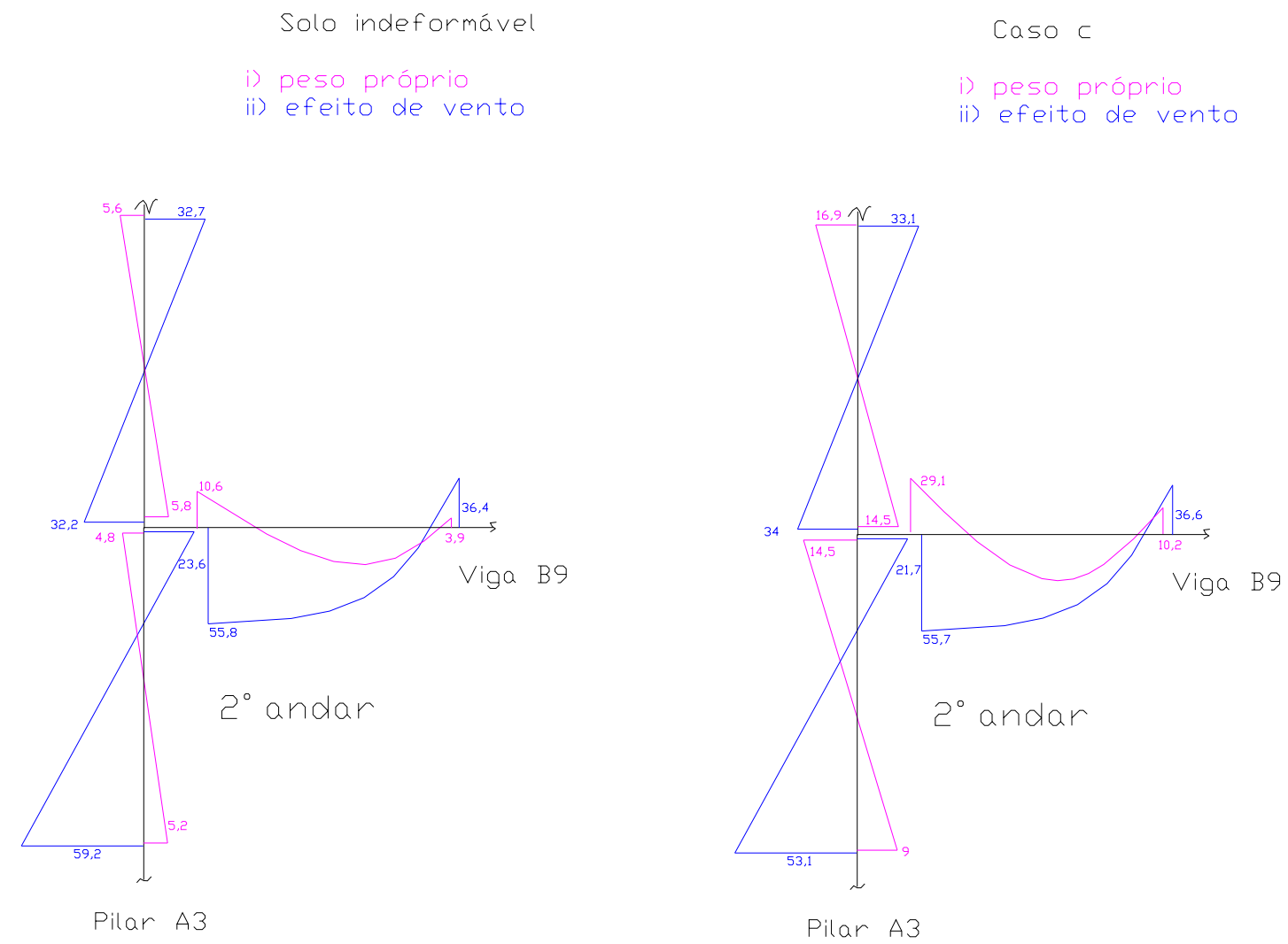

Figura 5.17 - Distribuição de momentos fletores $(\mathrm{kN} \cdot \mathrm{m})$ para um pilar ao longo de dois andares e de uma viga em comum a eles. 


\subsection{Uma estaca no meio finito homogêneo}

Este exemplo foi extraído de OTTAVIANI (1975), que analisa as respostas de deslocamentos e de tensões de contato entre a estaca e o solo empregando o MEF com elementos tridimensionais. Para avaliar este problema, o autor discretiza uma região prismática de modo que a superfície lateral região considerada não perturbável - está localizada a uma distância de $13 \mathrm{~m}$ do centro da estaca. Ele emprega uma rede com 3300 nós e 2700 elementos finitos do tipo "brick" formado por 8 nós.

O exemplo tem objetivo de comparar os resultados do autor citado com os obtidos pela presente formulação. Avalia-se, então, o efeito que as diferentes profundidades entre a base rígida e o final da estaca tem sobre o conjunto estaca/solo. Além disso, analisa-se, também, a influência que a relação de rigidez entre estaca/solo ( $\left.\lambda=E_{\text {estaca }} / E_{\text {solo }}\right)$ causa no conjunto.

A figura 5.18 apresenta a configuração do problema e os valores físicos e geométricos dos materiais envolvidos. As figuras $5.18 \mathrm{c}$ indicam as redes empregadas para discretizar o sistema estaca/solo. Neste caso, avaliaram-se as respostas para a variação da relação entre o diâmetro da região discretizada para o solo e o diâmetro da estaca. Para a combinação de redes mais densa do solo e da estaca, redes $\mathrm{c} 1 \mathrm{com}$ d1, o número total de nós gerados foram de 571 e de elementos de 820. E a discretização menos refinada foi o emprego das redes c 3 e d3, com 186 nós e 274 elementos triangulares planos.

A figura 5.19 apresenta os valores de força de contato cisalhante entre a estaca e solo na direção paralela ao eixo da estaca, para diferentes combinações de espessura de camada do solo e diferentes relações de rigidezes entre os dois meios. Nota-se que a influência da posição do plano indeslocável causa pouca variação dos resultados de forças de contato na região de contato entre estaca/solo, por outro lado, a relação entre a rigidez da estaca e do solo é bem mais significativo na distribuição dessas forças no fuste.

A figura 5.20 compara as respostas de deslocamentos entre as duas formulações, para as diferentes combinações entre espessura e relação de 
rigidez. As diferenças entre as duas formulações ficaram em torno de 5\% para os pontos ao longo do fuste, demonstrando que a presente formulação é consistente.

As figuras 5.21 e 5.22 apresentam as respostas ao longo do fuste de, respectivamente, deslocamentos e forças de contato ambos na direção paralela ao eixo da estaca. Estes resultados são plotados para as 3 diferentes redes discretizada para o solo mantendo-se a rede d1 para a estaca . Para os deslocamentos - figura 5.21 - mostra-se que para a relação entre os diâmetros de 10 e 50, os valores estão bem próximos: erros de menos de $1 \%$; e estes divergem dos obtidos para o caso da relação ser de 500 em torno de 2,43\% e $4,62 \%$ para os pontos, respectivamente, de topo e de base da estaca.

Para o caso das forças de contato cisalhante, nota-se, pela figura 5.22, que as relações de diâmetro 500 e 50 levam a configurações muito próximas, e uma pequena alteração para o caso da rede c3.

As figuras 5.23 e 5.24 apresentam também os deslocamentos e as forças de contato, também na direção paralela da estaca, mas agora variando o refinamento da rede dos elementos de contorno para a estaca, comparando-se as redes $\mathrm{d} 2$ e $\mathrm{d} 3 \mathrm{com}$ a rede $\mathrm{d} 1$. Para o caso dos deslocamentos, foi averiguada uma diferença de $6,84 \%$ para os pontos de topo, e de $12,15 \%$ para os de base da estaca quando se emprega a rede $d 2$, e para a rede $d 3$, essas diferenças ficaram em $41,46 \%$ e $69,81 \%$ (vide figura 5.23 ).

A figura 5.24 apresenta as respostas de forças de contato cisalhantes paras essas diferentes discretizações da estaca. Neste caso, as variações entre as densidades de elementos usadas influenciam, principalmente, para as tensões de ponta da estaca, chegando-se a inverter o sentido da reação dessas tensões.

Em suma, a variação da relação entre os diâmetros estaca/solo é menos significativa do que a consideração de uma discretização melhor para os elementos de fundação. 


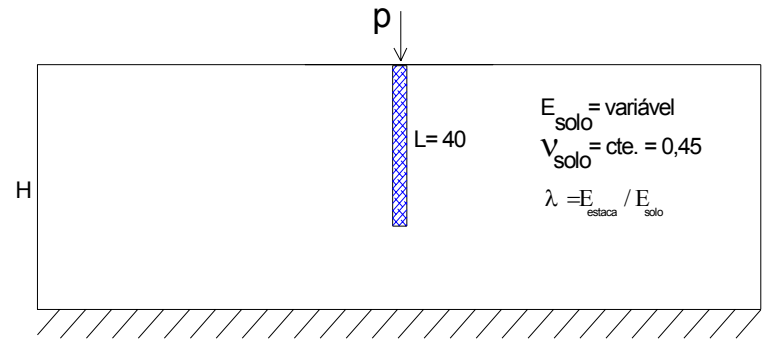

$D_{\mathrm{sol}}$

$$
\begin{aligned}
& \text { Estaca } \\
& \begin{array}{l}
\mathrm{E}=2,0 \mathrm{e} 5 \mathrm{kN} / \mathrm{m}^{2} \\
V_{\text {estaca }}=\text { cte. }=0,2 \\
\text { Diâmetro }=1 \mathrm{~m} \\
\mathrm{H} / \mathrm{L}=\text { variável } \\
\delta^{0}=\mathrm{E} \cdot \mathrm{D}_{\text {estaca }} \text {. W/P }
\end{array}
\end{aligned}
$$

a)

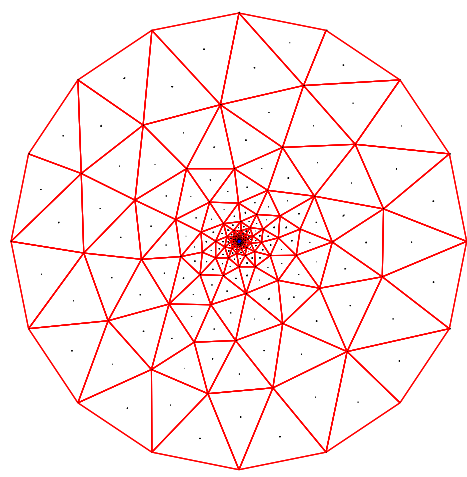

c1) $D_{\text {solo }} / D_{\text {estaca }}=500$

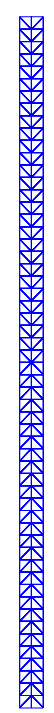

d1) 56 divisões:

456 elementos

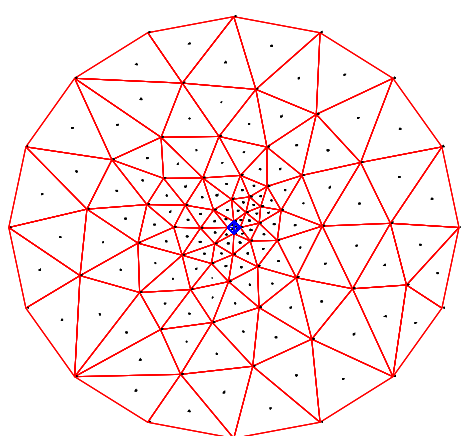

c2) $D_{\text {solo }} / D_{\text {estaca }}=50$
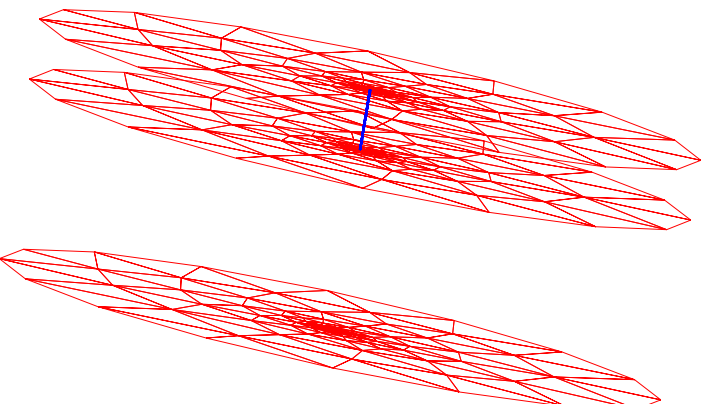

b)

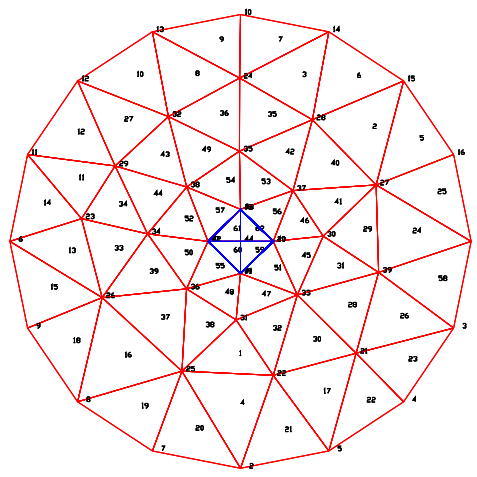

c3) $D_{\text {solo }} / D_{\text {estaca }}=10$

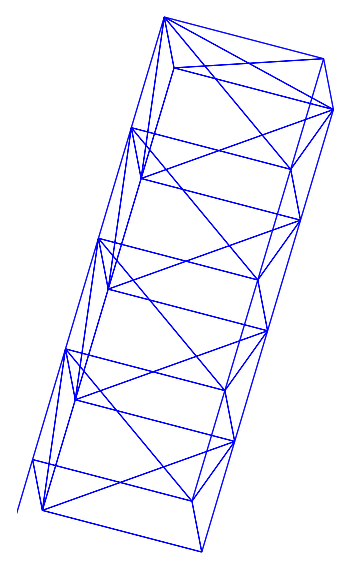

e)

Figura 5.18 - a) estaca sobre força vertical em meio indeslocável; b) perspectiva da discretização empregada; c) redes usadas para simular a superfície livre do solo; d) redes usadas para representar a estaca; e) uma perspectiva da rede da estaca. 


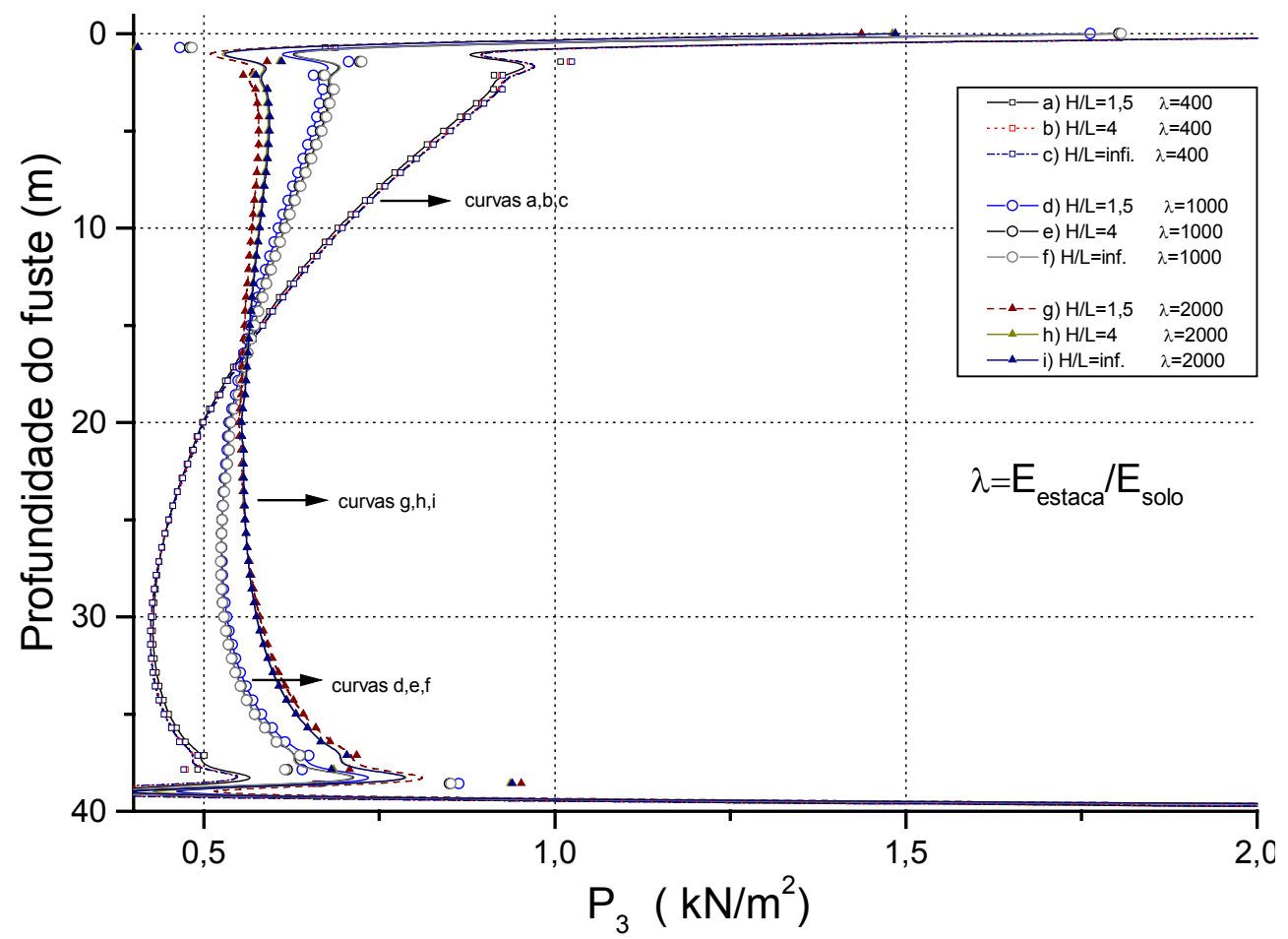

Figura 5.19 - Forças de contato cisalhante entre estaca/solo para diversos $\lambda$ e $\mathrm{H} / \mathrm{L}$, para a rede i/iv.

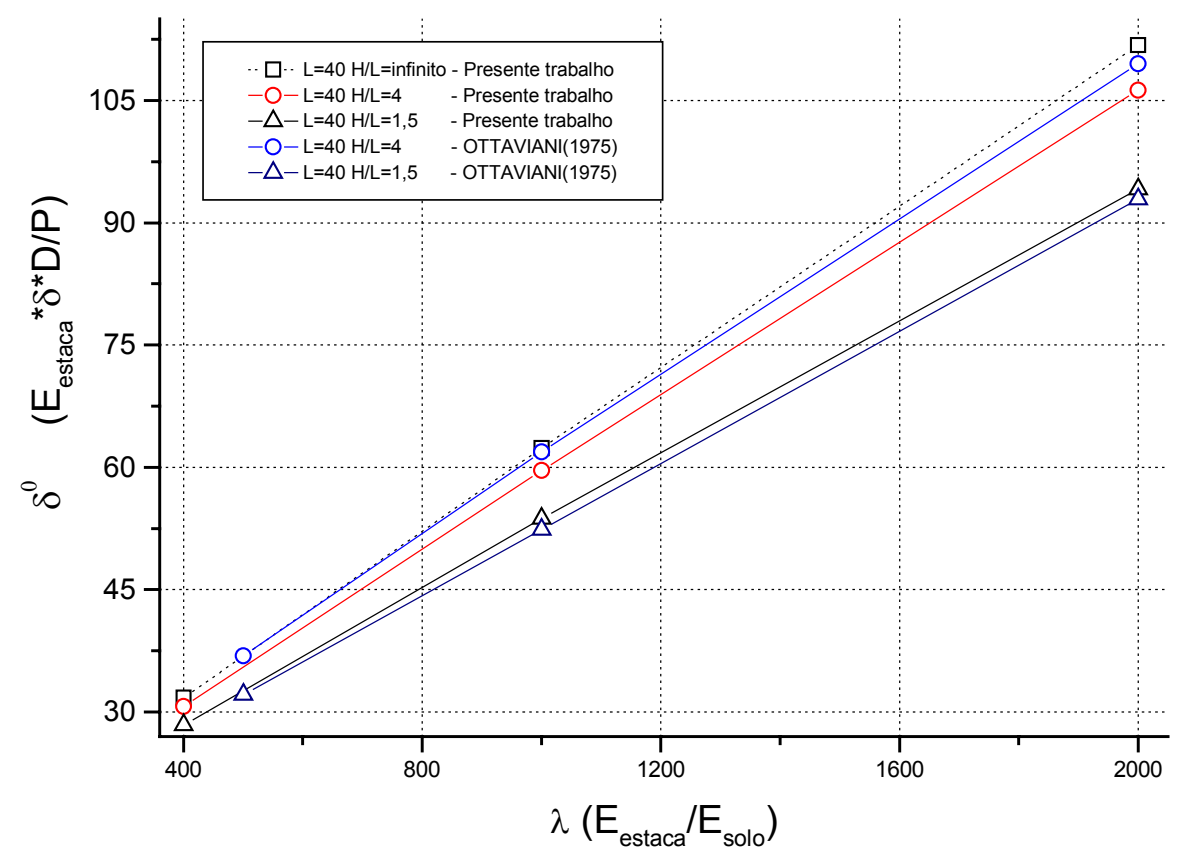

Figura 5.20 - Deslocamentos verticais das estacas para diversos $\lambda$ e H/L, para a rede i/iv. 


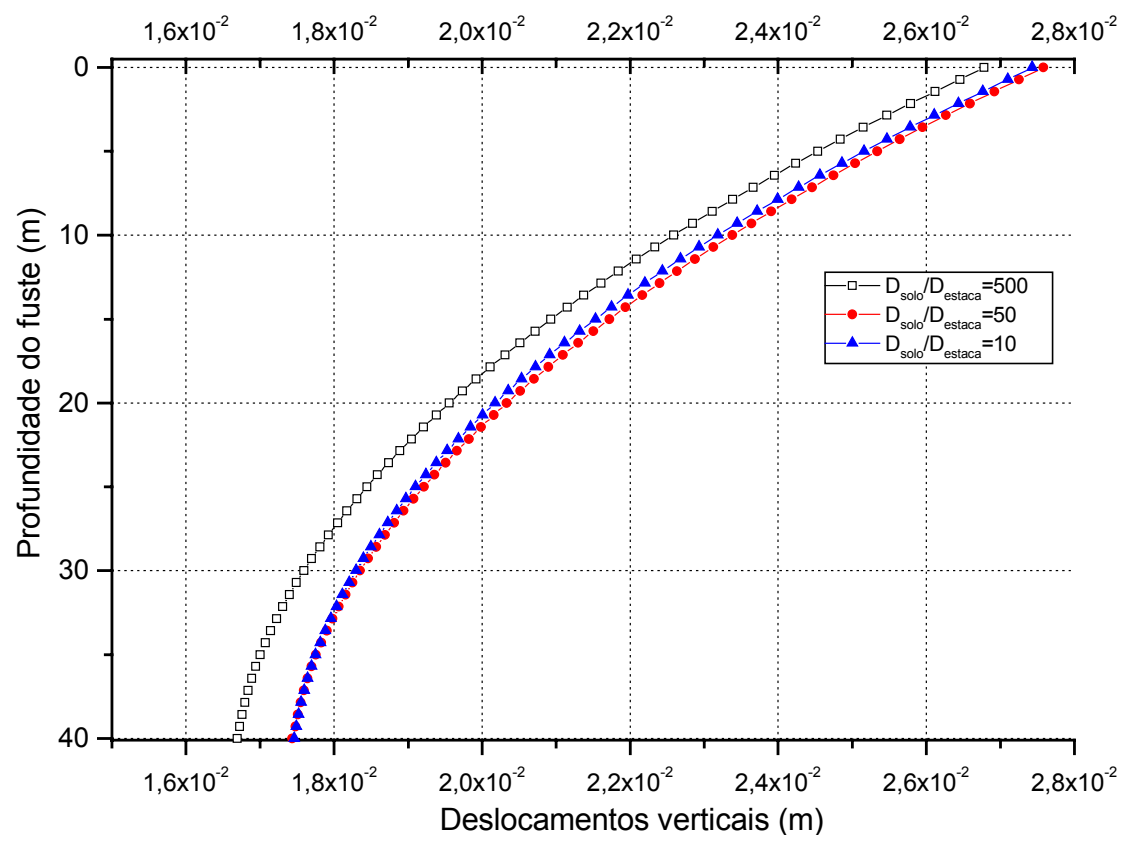

Figura 5.21 - Deslocamentos da estaca para os 3 tipos de redes do solo, empregando a rede iv, $H / L=1,5$ e $\lambda=1000$.

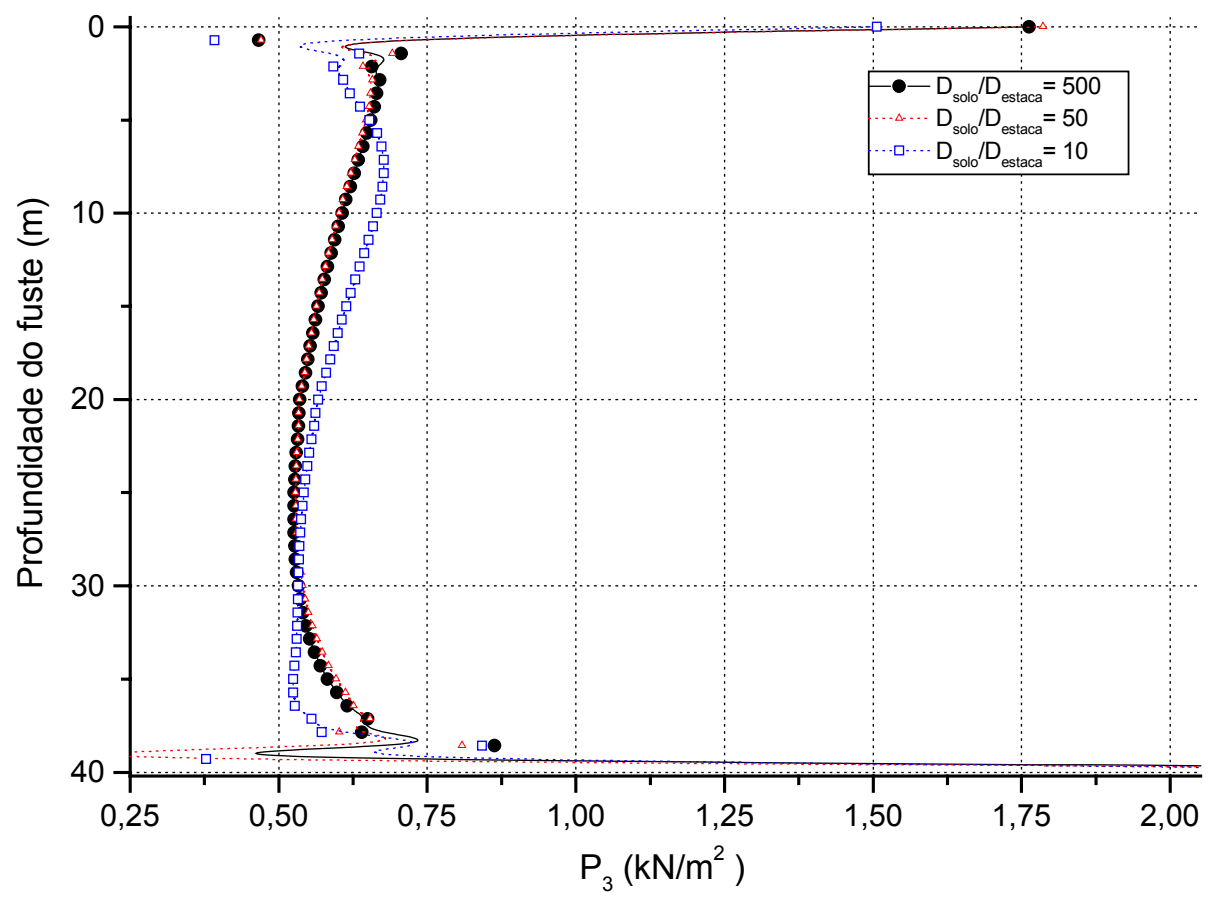

Figura 5.22 - Forças de contato cisalhante entre estaca/solo para os 3 tipos de redes, empregando a rede iv, $H / L=1,5$ e $\lambda=1000$. 


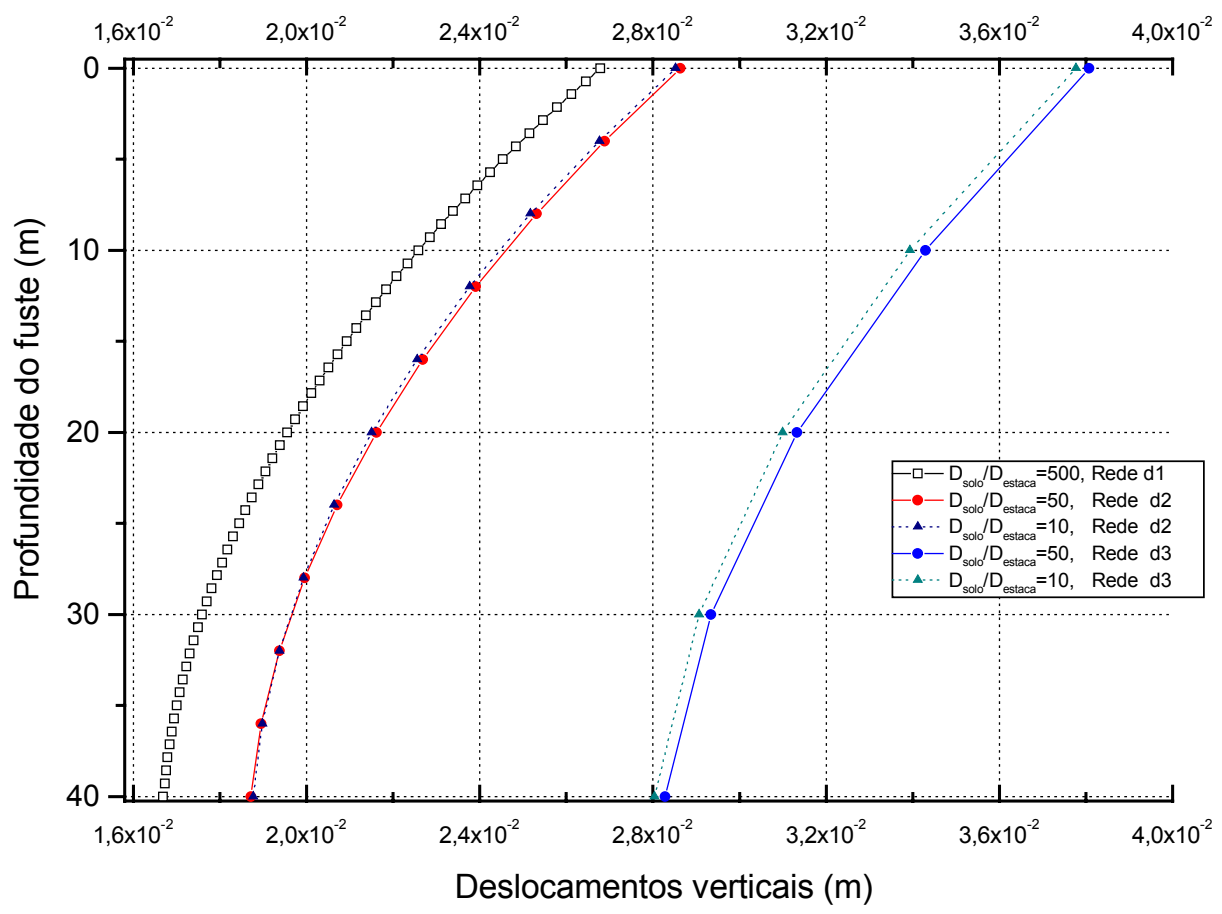

Figura 5.23 - Deslocamentos da estaca com os 3 tipos de redes do solo, empregando as 3 redes da estaca, para $\mathrm{H} / \mathrm{L}=1,5$ e $\lambda=1000$.

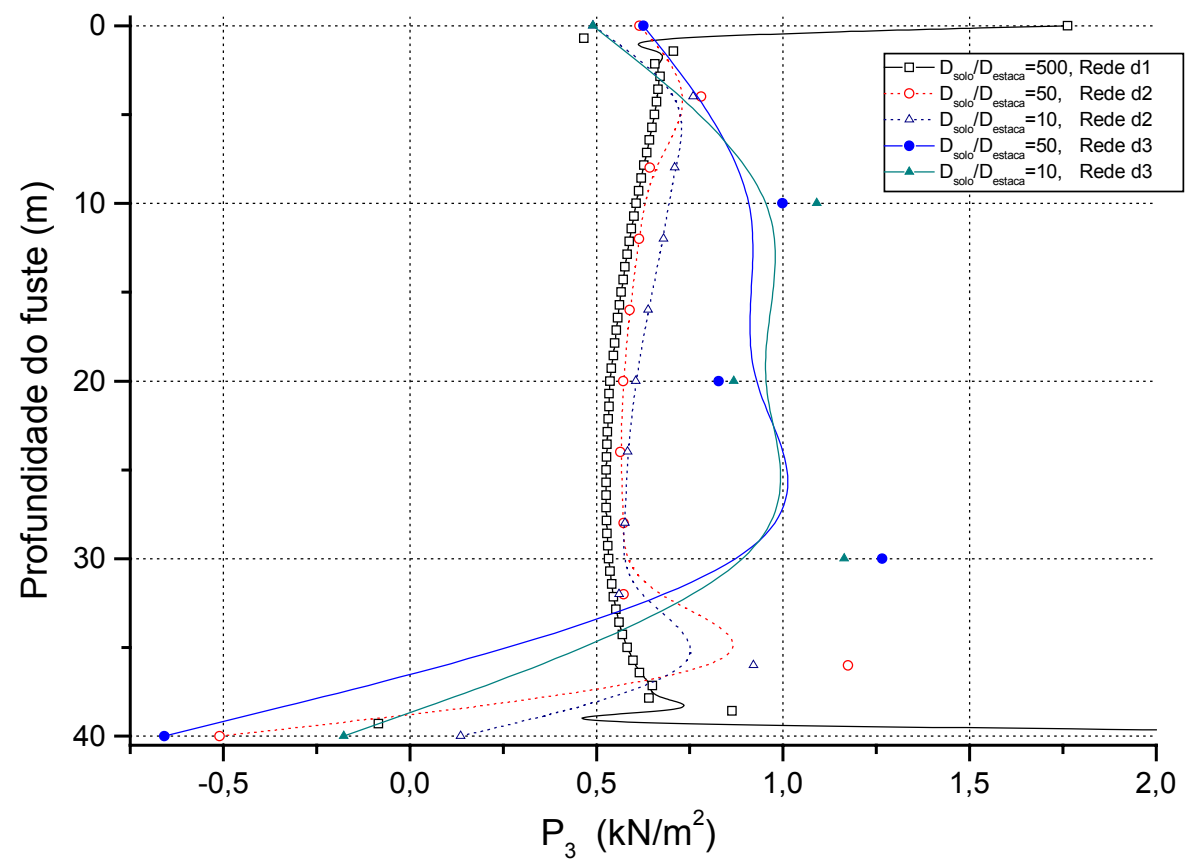

Figura 5.24 - Forças de contato cisalhante entre estaca/solo para os 3 tipos de redes, empregando as 3 redes da estaca, para $H / L=1,5$ e $\lambda=1000$. 


\subsection{Uma estaca no meio finito não-homogêneo}

O exemplo apresenta a distribuição de deslocamentos e de tensões verticais ao longo do fuste da estaca, a qual esta imersa em um meio nãohomogêneo. Assim, emprega-se a rede de elementos para discretização do solo e da estaca usada no exemplo anterior, figura 5.18. Para o solo é utilizada a rede c2 e para a estaca a rede d1. A figura 5.25 apresenta a configuração do problema analisado, os valores das variáveis envolvidos e os casos considerados para a consideração da não homogeneidade do maciço.

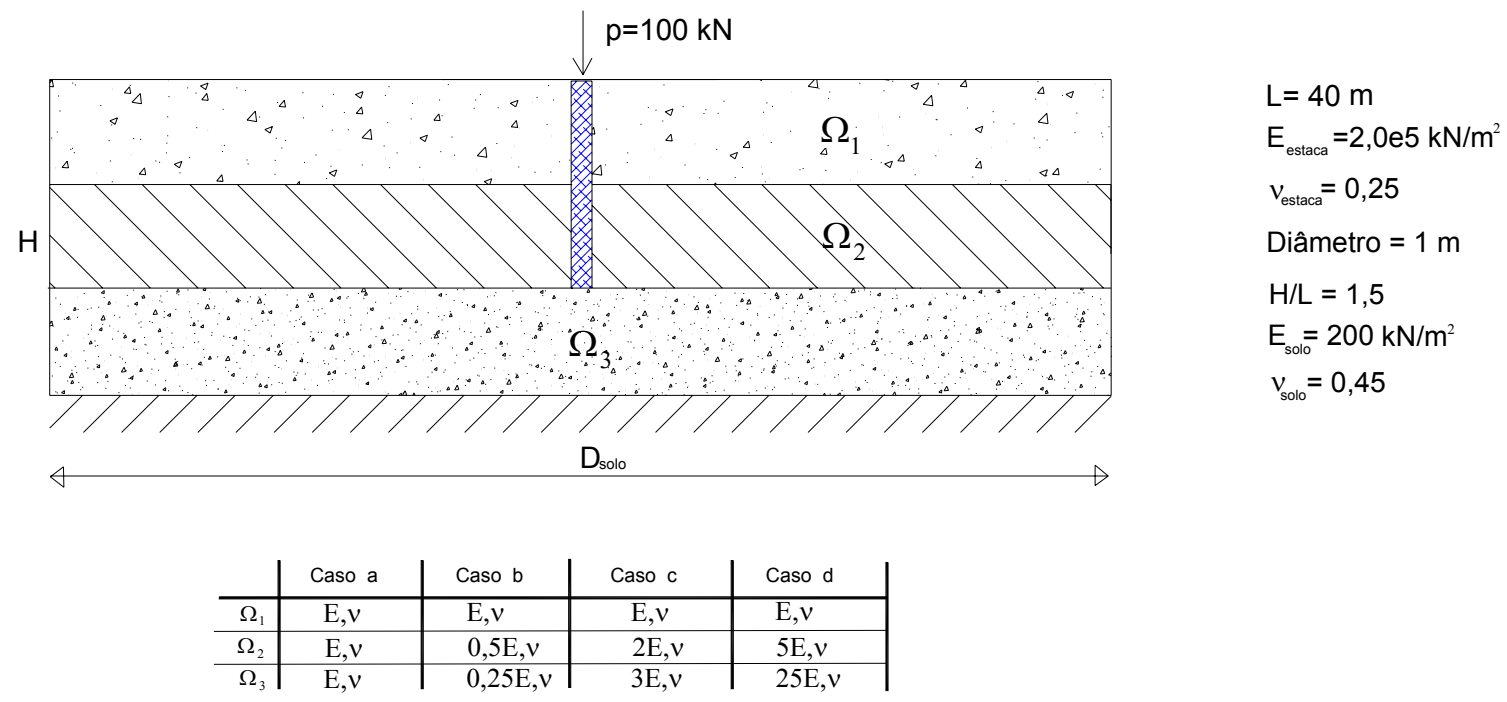

Figura 5.25 - Estaca sujeita a força vertical em meio não-homogêneo e configurações do maciço.

A figura 5.26 compara as forças cisalhantes verticais de contato entre o maciço e a estaca, quando essa última esta imersa totalmente em uma região do solo, ou quando se divide essa região em duas camadas, assim tem-se um maciço com três camadas ao todo. É mantido o módulo de elasticidade constante para todo o meio, de maneira que as duas situações simuladas devem tender aos mesmos valores. Isso foi feito para verificar a consistência da formulação quando se estratifica o solo com a presença de fundação. Nota-se que as diferentes representações do mesmo problema levam a uma mesma 
resposta de forças, ressaltando que na região de contato entre os dois meios, há uma pequena oscilação nos valores dessas tensões. Entretanto, é fato que essa perturbação é muito pequena e, principalmente, não afeta o comportamento dos valores ao longo do fuste. Foi notado que quando se empregou a rede c3 para representar o solo, essa oscilação foi bem marcante e, a medida que foram sendo utilizados as redes c2 e c3, essa perturbação foi se atenuando. Para o caso dos deslocamentos, o emprego dessas diferentes simulações não causou estes pequenos saltos próximos à região de contato entre as duas sub-regiões. Para o emprego da rede $\mathrm{c} 1 \mathrm{com}$ a rede $\mathrm{d} 1$, a diferença de deslocamentos do topo da estaca usando uma ou duas regiões foi de $0,34 \%$.

As figuras 5.27 e 5.28 apresentam os resultados ao longo do fuste de deslocamentos e forças de contato cisalhante verticais para os quatro tipos de combinações de rigidez para o maciço.

Pela figura 5.27, nota-se que realmente os deslocamentos verticais apresentam um comportamento contínuo ao longo das diferentes sub-regiões. As diferenças relativas de deslocamentos de topo entre o caso a e os demais casos são de: $-27,35 \%$ para o caso $b, 46,00 \%$ para o caso $c$, e de $-48,15 \%$ para o caso $d$.

A figura 5.28 apresenta os gráficos para as forças de contato verticais entre estaca/solo, e é possível verificar uma expressiva diferença entre as várias situações, onde para o caso a os valores ficam constantes em torno de $0,6 \mathrm{kN} / \mathrm{m}^{2}$. Para os demais casos, os valores se elevam nas regiões de maior rigidez e se mantêm constante dentro desta. Nota-se que a variação dos valores existentes entre uma camada e outra, para um mesmo caso, é em torno de $100 \%$. Para os pontos próximos a ponta, estes valores são mais elevados para os casos onde a rigidez do solo em contato com a ponta da estaca é maior. As moderadas diferenças obtidas entre os valores de deslocamentos e de forças de contato verticais para estes diferentes casos demonstram que a consideração do maciço de forma mais próxima de seu natural, ou seja, 
mediante a inclusão dessa heterogeneidade, é uma condição obrigatória para a análise mais criteriosa de um projeto que envolva a interação solo/fundação/superestrutura.

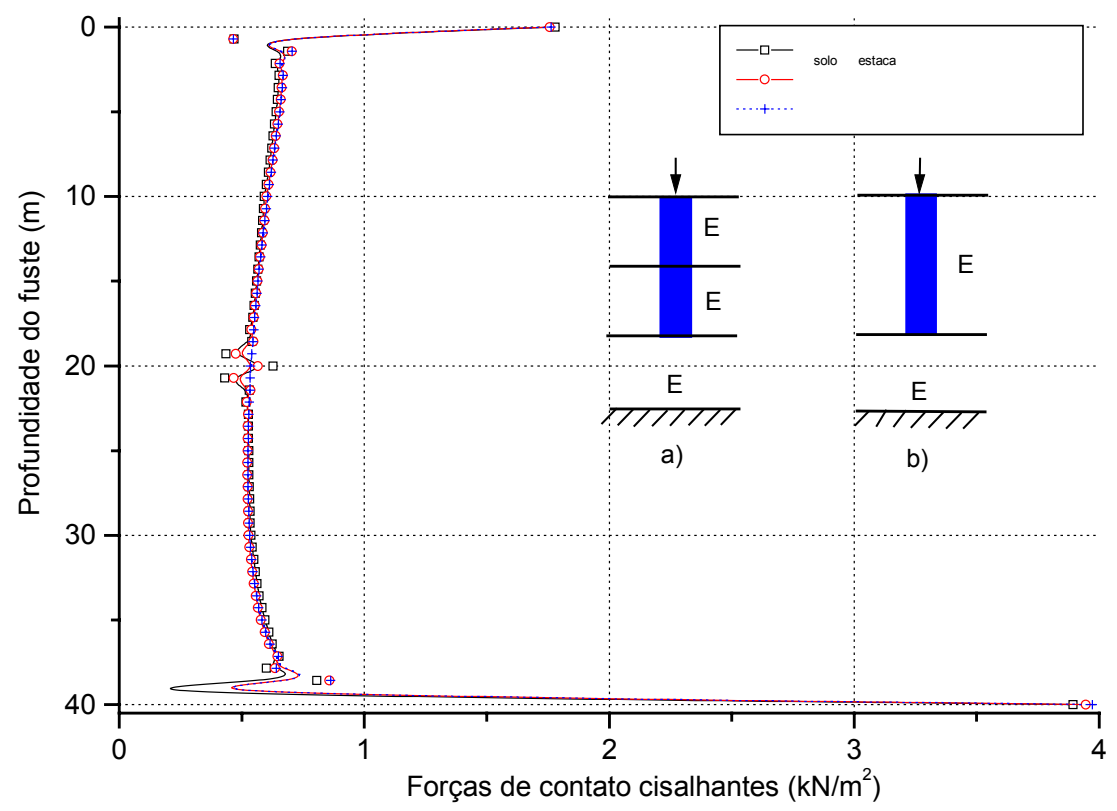

Figura 5.26 - Forças de contato verticais entre estaca/solo para diferentes estratificações.

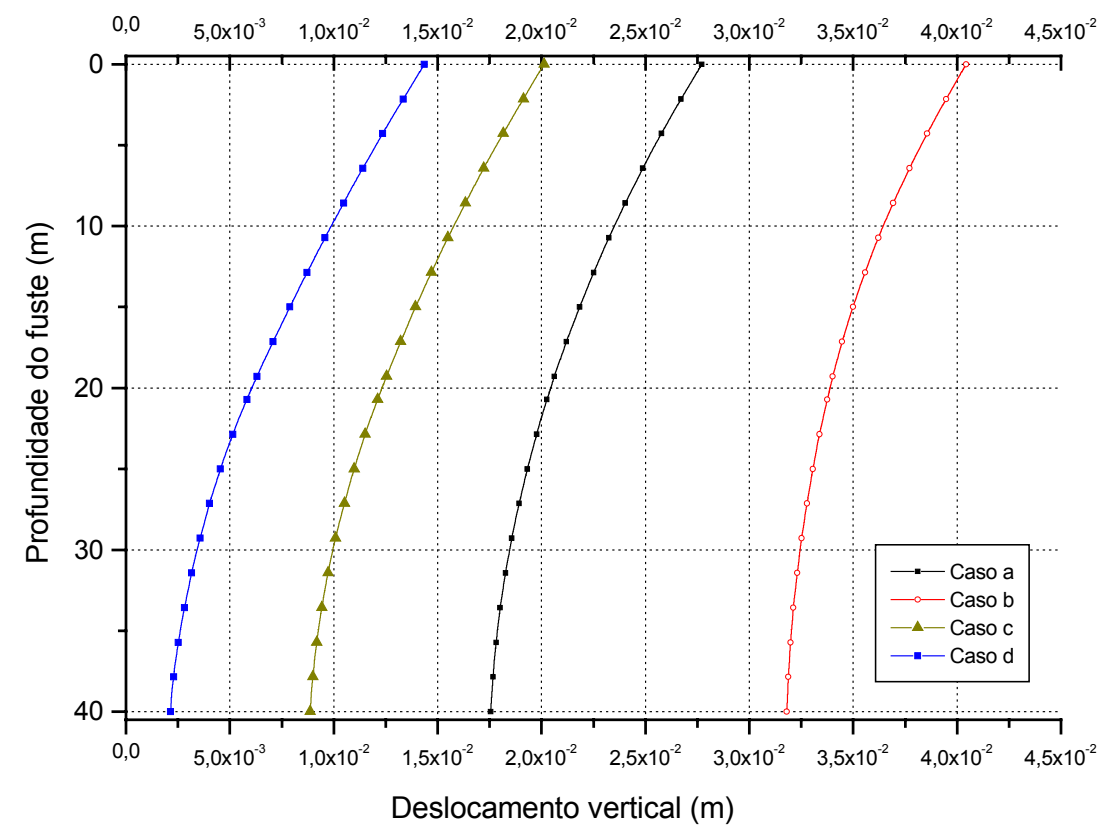

Figura 5.27 - Deslocamentos verticais na estaca para 4 casos de rigidez do solo. 


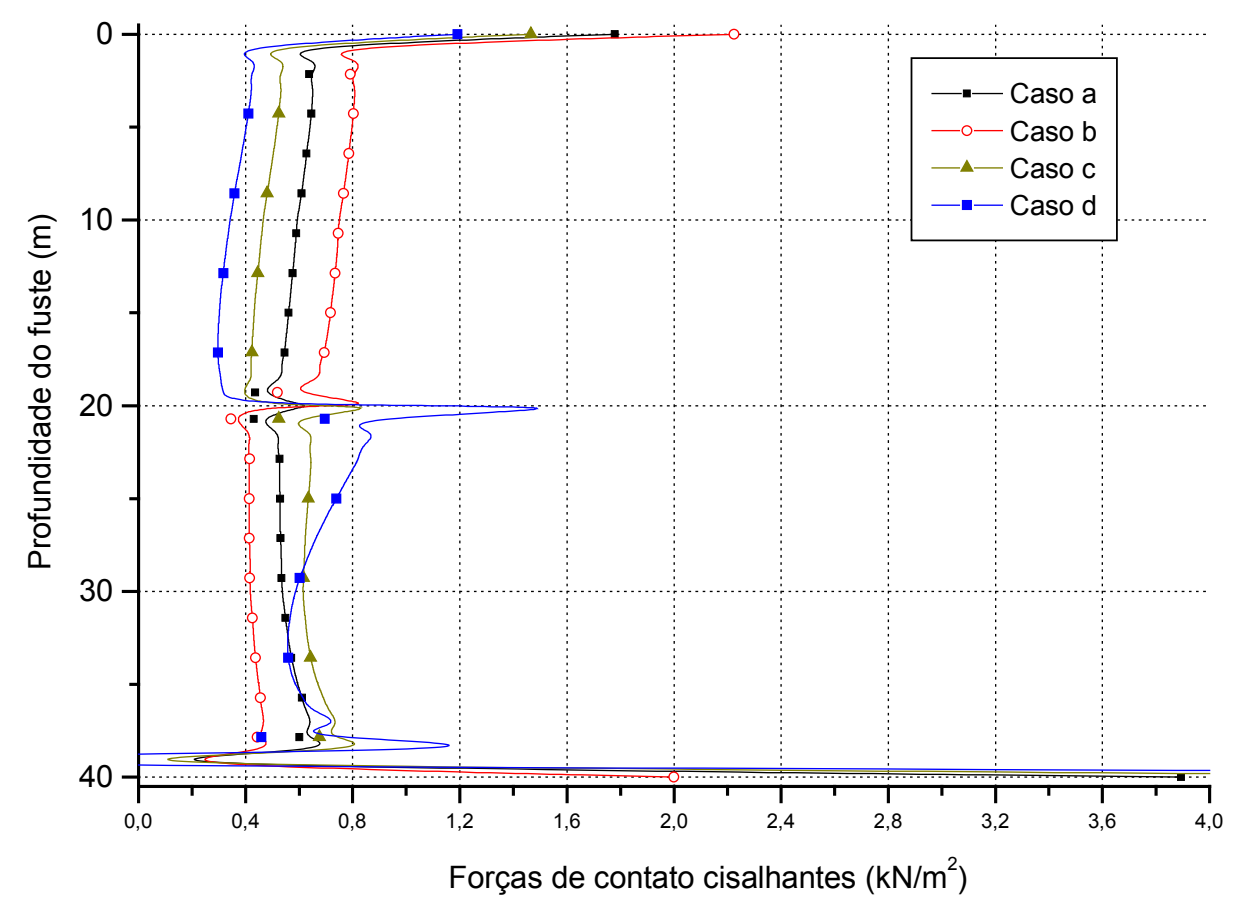

Figura 5.28 - Forças de contato cisalhantes verticais entre estaca/solo para 4 casos de rigidez do solo.

\subsection{Lâmina quadrada apoiada sobre nove estacas}

Este exemplo, também retirado de Ottaviani (1975), apresenta uma lâmina em contato com o meio finito com nove estacas distribuídas conforme figuras 5.29 e 5.30. Na figura 5.29 são mostradas as posições das estacas e do plano indeslocável e as características físicas e geométricas da lâmina, das estacas e do solo.

A figura 5.31 mostra os deslocamentos da superfície do solo ao longo do corte $A B$ ocorridos quando se aplica uma força distribuída uniformemente constante em sua superfície quadrada. O módulo de rigidez da lâmina é variado para se verificar o comportamento desses deslocamentos. Assim, cinco casos do módulo de Young da lâmina são avaliados, e nota-se que quando o quociente entre o módulo da lâmina e do solo está acima de $10^{6}$, o comportamento da lâmina é de uma estrutura rígida. 
As figuras 5.32 e 5.33 apresentam resultados de deslocamentos e forças de contato paralelo ao eixo das estacas, sem a consideração da lâmina sobre o maciço. As estacas são mobilizadas via forças concentradas com valores de 100 kN. No gráfico da figura 5.33, vê-se a distribuição das forças de contato para as estacas 3, 5 e 7, mostrando que os resultados obtidos nas estacas $3 \mathrm{e}$ 7 são simétricos. A figura 5.32 plota os valores de deslocamentos ao longo do fuste para essas três estacas, e para a estaca 5 são comparados os valores de topo e base com os apresentados em OTTAVIANI (1975), e as diferenças ficaram em torno de $10 \%$. Os resultados de deslocamentos retirados em OTTAVIANI (1975) são dados apenas para o topo e a base, sendo então ligados por uma função linear ao longo do fuste.
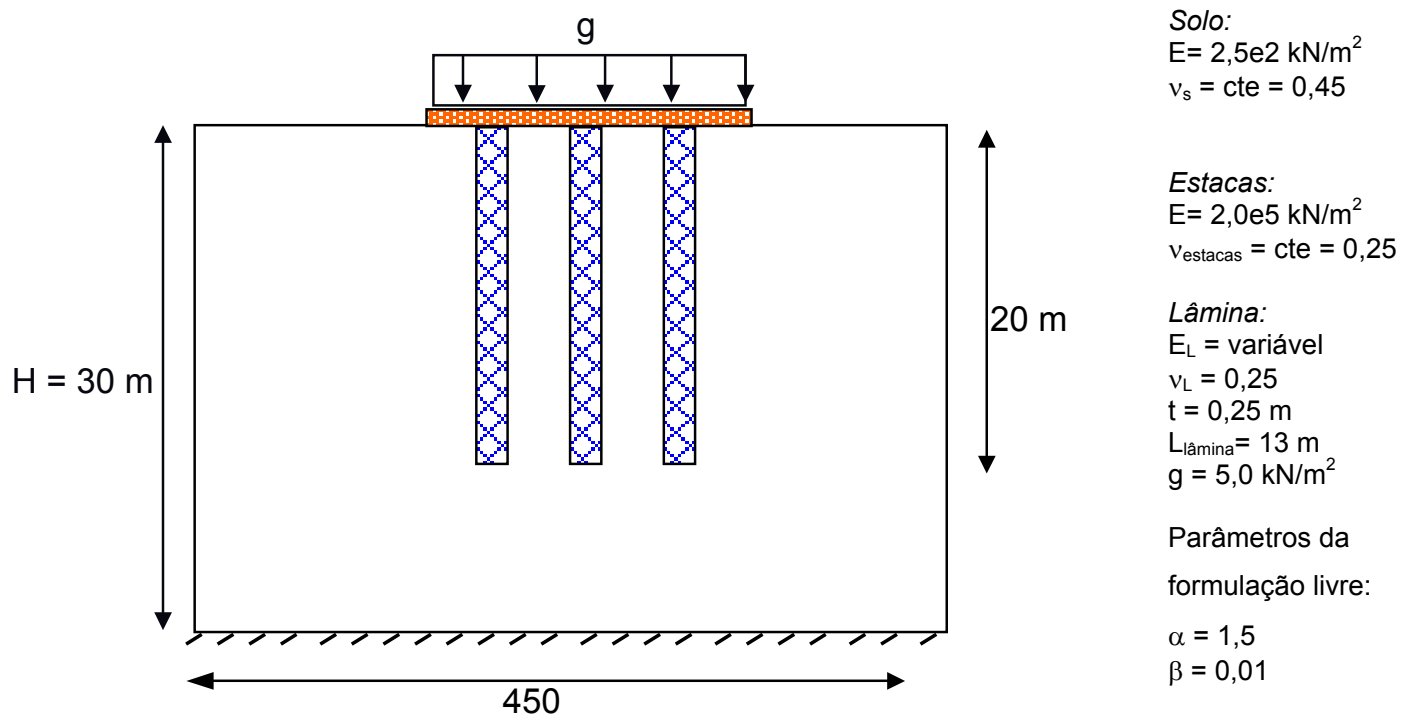

Figura 5.29 - Representação da lâmina sobre maciço homogêneo estaqueado apoiado em meio indeslocável, e seus parâmetros físicos e geométricos. 

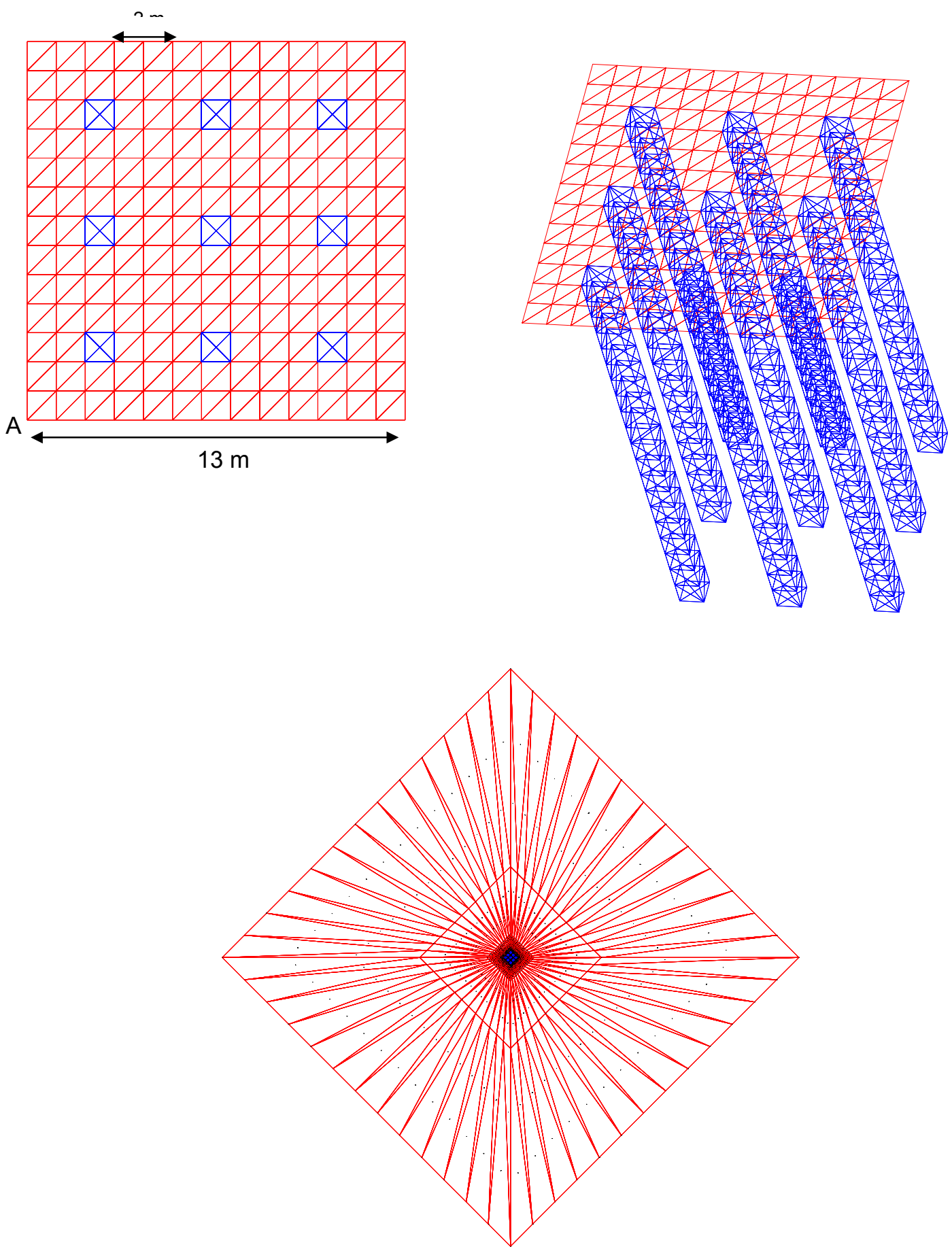

Figura 5.30 - Redes usadas para discretizar a lâmina, as estacas e a superfície do solo. 


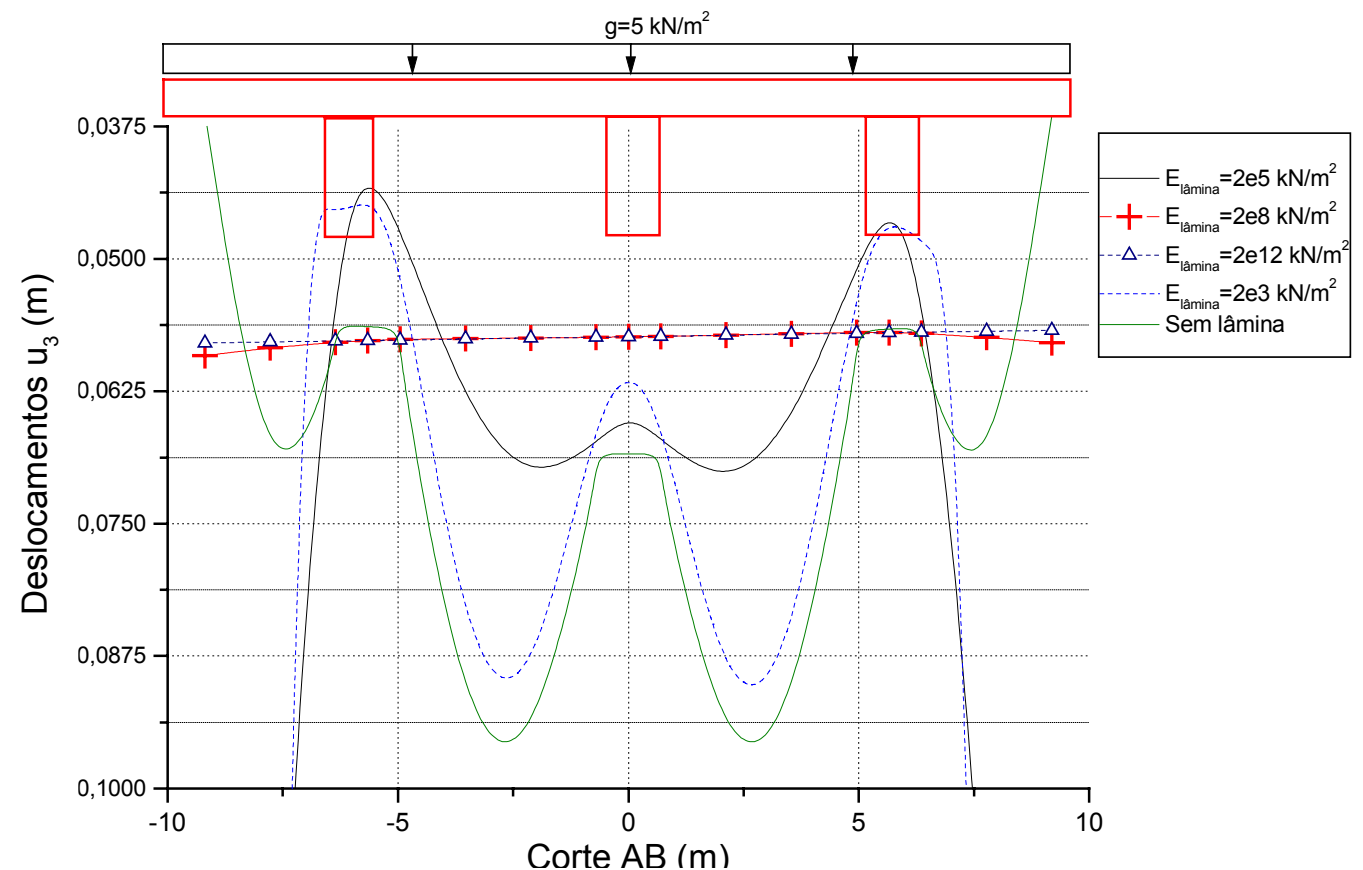

Figura 5.31 - Deslocamentos da superfície de contato lâmina/solo/estaca.

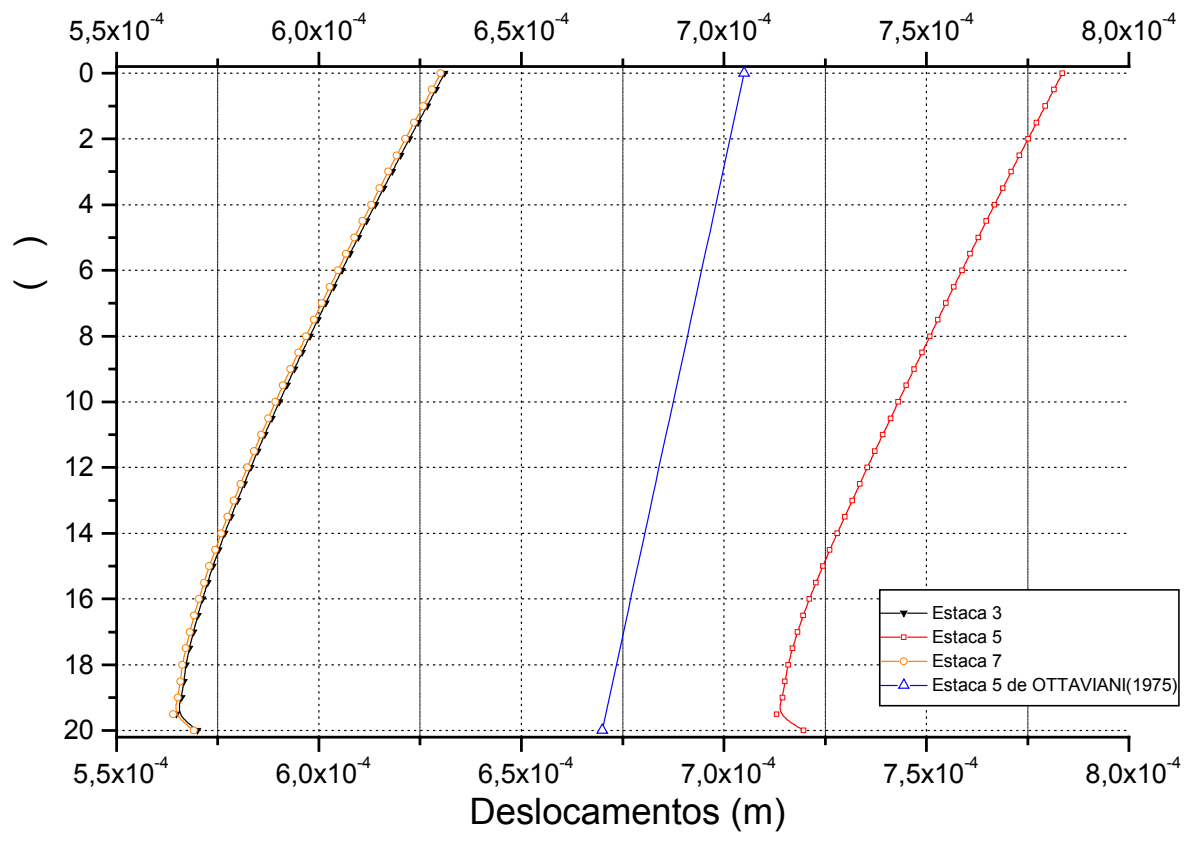

Figura 5.32 - Deslocamentos das estacas ao longo do fuste sem lâmina. 


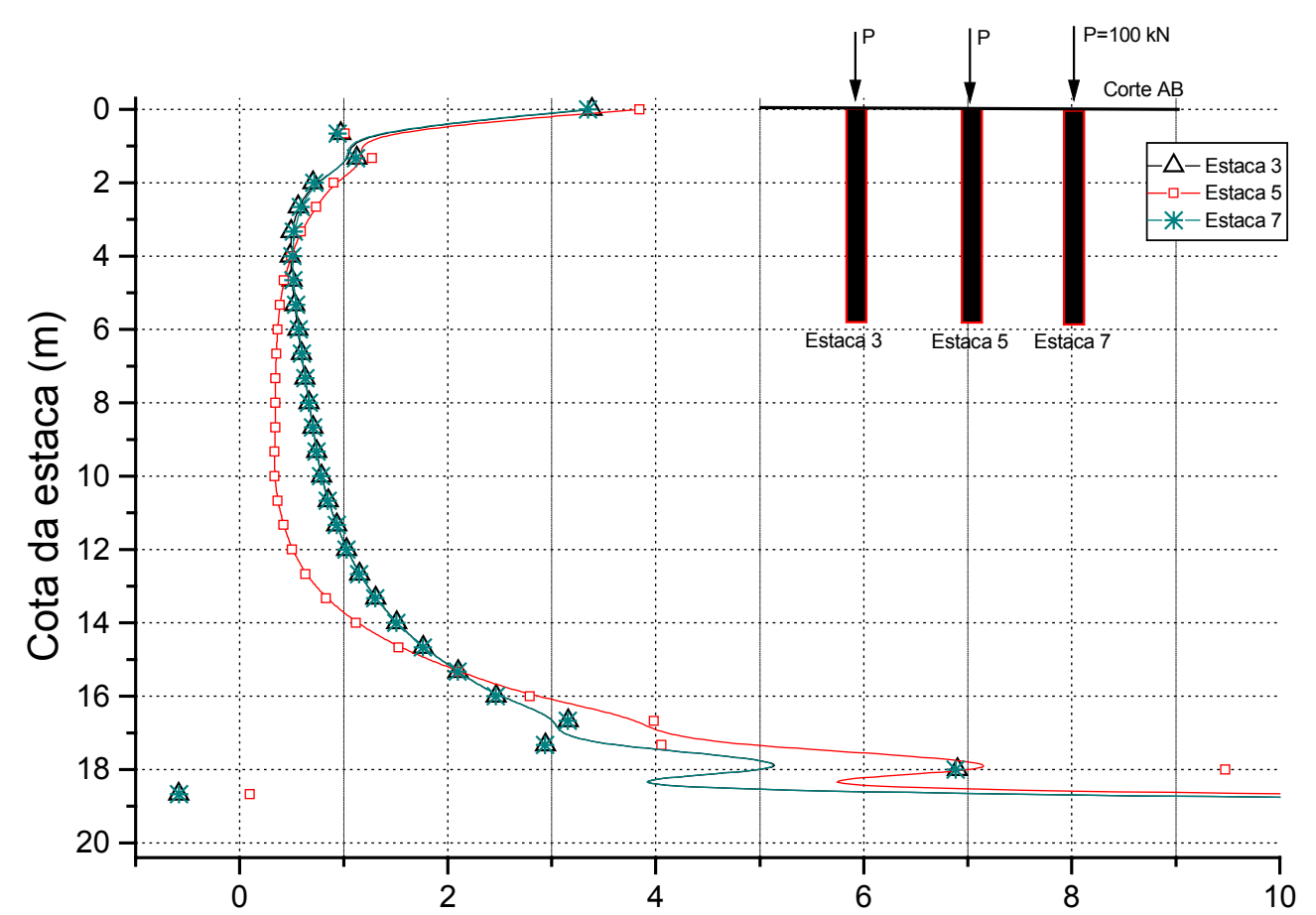

Figura 5.33 - Forças de contato cisalhantes entre solo/estaca sem lâmina.

\subsection{Cobertura apoiada em "radier" com e sem estacas}

Este exemplo tem o intuito de comparar os resultados obtidos em uma cobertura para duas situações: i) quando se varia a posição do plano indeslocável sem a inclusão de estacas, e ii) quando se varia a posição do plano indeslocável com a inclusão das estacas. A figura 5.34 apresenta a configuração e os parâmetros físicos e geométricos necessários do problema.

Para o primeiro caso, variou-se as espessuras da camada deformável do solo de forma a se verificar essa influência na cobertura. Assim, a figura 5.35 apresenta os valores de deslocamentos da superestrutura obtidos ao longo de um corte paralelo ao eixo $\mathrm{X}_{1}$, onde as forças concentradas aplicadas na cobertura possuem valores de $\mathrm{P}=30 \mathrm{kN}$ (total de $120 \mathrm{kN}$ ). A espessura do solo com o mesmo tamanho que a lâmina $(\eta=1)$ demonstra ser o ponto determinante para se considerar a deformabilidade do maciço para a análise de recalques da cobertura (vide figura 5.35). Pois, para os casos onde se aplicam os fatores de espessura zero $(\eta=0)$ e um $(\eta=1)$, os valores de deslocamento do centro da 
cobertura são de, respectivamente, $0,73 \mathrm{~mm}$ e $2,39 \mathrm{~mm}$, e quando se considera o fator de espessura de valor dois $(\eta=2)$, o deslocamento se eleva para $22,64 \mathrm{~mm}$. A partir dessa espessura, este valor cresce de maneira lenta, de modo que, no limite superior, quando o meio está apoiado sobre o plano indeslocável a distâncias infinitas $(\eta=\infty)$, este valor é de $29,95 \mathrm{~mm}$.

Para o caso de se considerar a cobertura apoiada sobre o radier estaqueado, empregou-se este mesmo exemplo, mudando-se os valores das forças concentradas, passando essas para $P=10 \mathrm{kN}$ (total de $40 \mathrm{kN}$ ), a espessura do solo é de $30 \mathrm{~m}(\eta=2,31)$, e as estacas têm comprimento de $20 \mathrm{~m}$. Assim, na figura 5.36 plota-se os valores de deslocamentos mobilizados quando da aplicação dessas forças concentradas na região central da cobertura, quando se considera ou não a compressibilidade do solo.

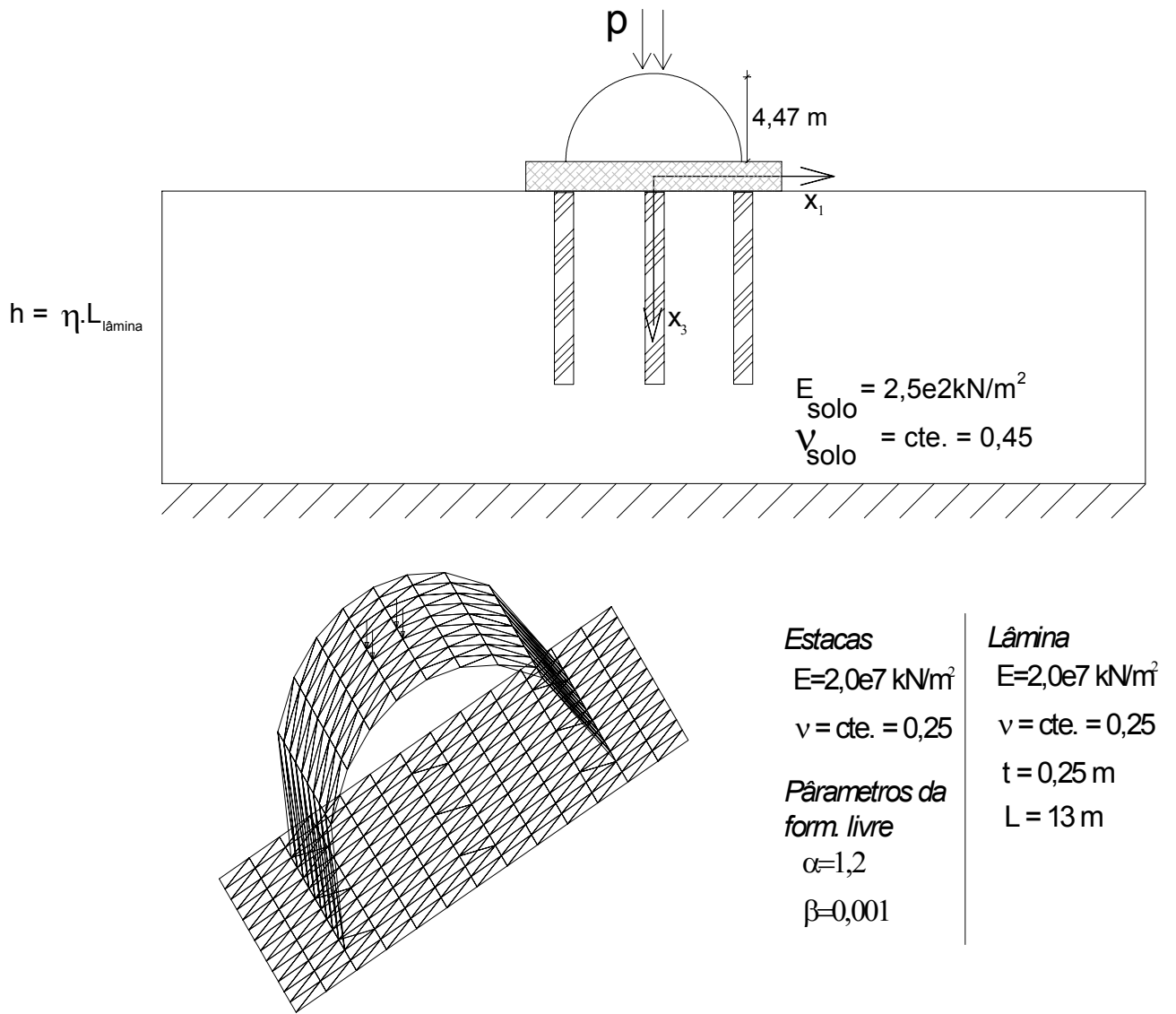

Figura 5.34 - Representação da cobertura sobre maciço homogêneo estaqueado. Rede empregada para o solo idem a figura 5.22 . 


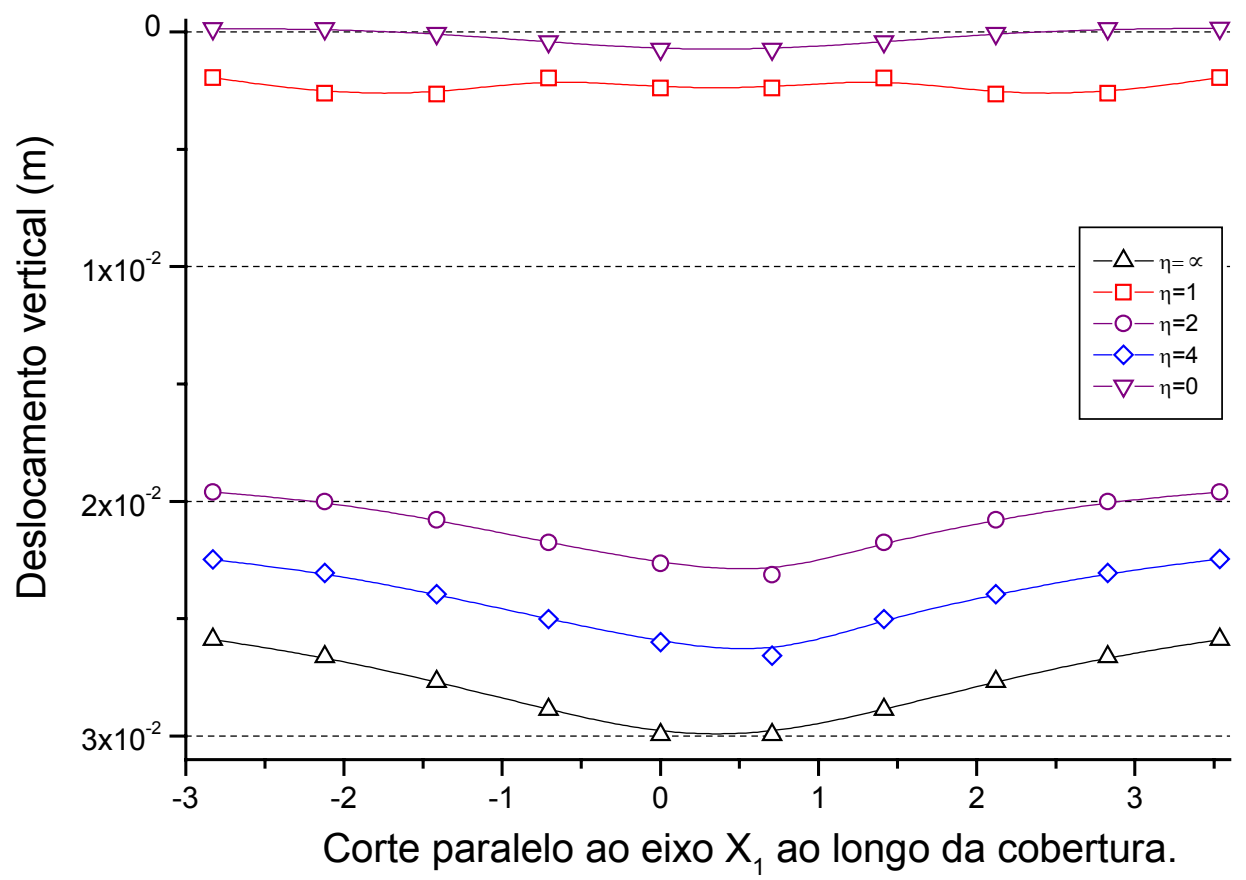

Figura 5.35 - Deslocamento vertical na seção da cobertura paralelo ao eixo $\mathrm{X}_{1}$ para diferentes fatores de espessura do solo, sem estacas.

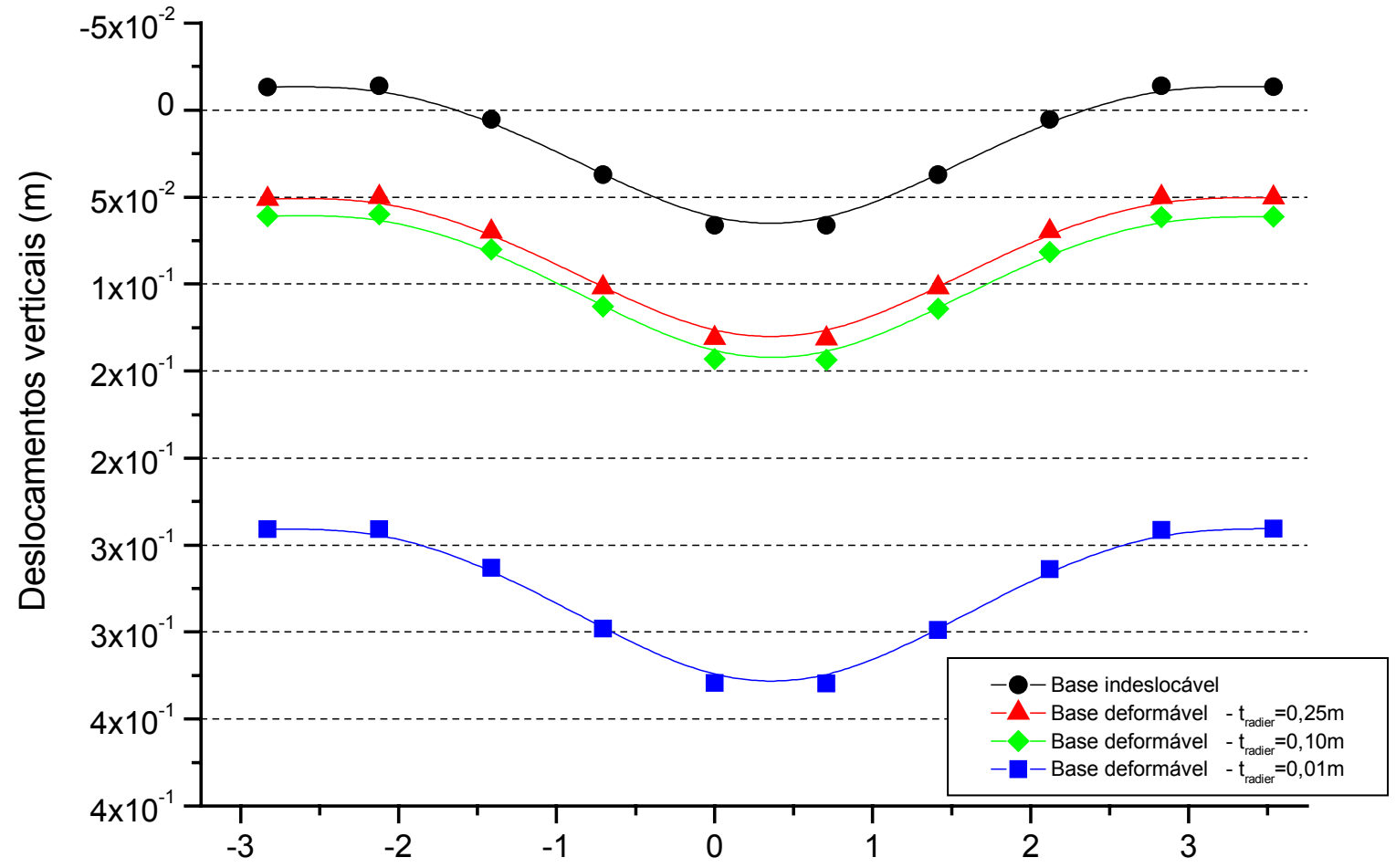

Figura 5.36 - Deslocamentos da cobertura no corte CD considerando diversas espessuras do radier, com o solo estaqueado. 


\section{Capítulo 6}

\section{PARALELISMO E RESOLUÇÃO DE SISTEMAS ESPARSOS}

\subsection{Introdução}

Este tópico tem o intuito de apresentar sucintamente duas ferramentas numéricas que foram empregadas para agilizar a análise numérica. Como os modelos numéricos no campo da mecânica computacional têm se tornando muito complexos, em virtude do avanço dos componentes eletrônicos ligados aos computadores, torna-se imperativo o uso dessas ferramentas para que as soluções sejam obtidas em tempos menores.

Neste sentido, no item 6.2 é desenvolvido um texto que trata da aplicação do processamento paralelo no campo da análise numérica. Este texto aborda o assunto de maneira bem direta e breve, sendo que nos trabalhos de ALMEIDA \& PAIVA (2000), ALMEIDA (1999) e TOPPING \& KHAN (1996) esse assunto é tratado de forma mais geral e didática.

O item 6.3 aborda o tema da resolução de sistemas esparsos. Comentam-se, então, as vantagens e as desvantagens de se empregar os métodos diretos e iterativos para este tipo especial de matriz. No final, mostrase um exemplo numérico onde se empregam essas duas diferentes técnicas de resolução de sistemas. 


\subsection{Computação distribuída}

Computação distribuída é o processo no qual um conjunto de computadores são conectados por uma rede e usados coletivamente para solucionar um problema extenso. Para o caso de se usar computadores individuais com suas próprias memórias, estes sistemas são chamados de multicomputadores.

Os últimos anos têm testemunhado um avanço da aceitação e adoção do processamento paralelo, ambos para a computação científica de alta performance e para as aplicações em "propósitos genéricos" - resultado da exigência para mais alta performance, mais baixo custo e produtividade sustentada. A mecânica computacional foi um meio em que esta ferramenta se mostrou bastante eficiente e prática para a análise de grandes sistemas mecânicos via os métodos numéricos.

Pois, cada vez mais, os problemas estão sendo analisados detalhadamente para permitir uma melhor simulação do real comportamento do material e também a simulação dos parâmetros do modelo e a respectiva análise de suas respostas. Isso tem exigido a geração de complexos modelos numéricos que avaliem grandes quantidades de dados e que exijam grande tempo de execução.

No contexto histórico, a partir do início da década de 60, os algoritmos numéricos começaram a ser desenvolvidos de forma a serem aplicados não somente em máquinas de arquiteturas seqüenciais, mas também em paralelas. A comunidade científica e a engenharia se dedicaram ao estudo e à implementação de novos procedimentos para explorar estas novas arquiteturas. Citam-se os trabalhos de CARROLL \& WETHERALD (1967) e LEHMAN (1966), que fizeram um estudo inicial do uso dessas técnicas para tais máquinas.

$\mathrm{Na}$ metade da década de 70 , trabalhos mais aplicados à engenharia foram desenvolvidos, como o estudo pioneiro realizado por MIRANKER (1971), sobre algoritmos, na análise numérica em computação paralela, estudos estes 
de otimização matemática, encontro de raízes, equações diferenciais e solução de sistemas lineares.

Após este trabalho, o avanço nas aplicações - mediante principalmente o uso do método dos elementos finitos - começou a ser feito mais comumente explorando os novos paradigmas computacionais de forma a resolver seus problemas em processamento distribuído. Estes os trabalhos utilizaram os novos paradigmas: NOOR \& HARTLEY (1978), NOOR \& LAMBIOTTE (1979), ZOIS (1988a e 1988b), LAW (1986), BARRAGY \& CAREY (1988), NOOR \& PETERS (1989), GROSSO \& RIGHETTI (1988) JOHNSSON \& MATHUR (1990), ADELI \& KAMAL (1992), FARHAT \& ROUX (1991), SAXENA \& PERUCCHIO (1992) RAO et al. (1993), LAW \& MACKAY (1993), SCHMIT \& LAI (1994), BITZARAKIS et al. (1997) CHEN \& BYREDDY (1997).

Para a análise numérica em processamento distribuído de um problema mecânico usando-se um método discreto qualquer -Método das Diferenças finitas (MDF), Métodos dos Elementos Finitos (MEF) ou Método dos Elementos de Contorno (MEC) -, é necessário aplicar uma das duas diferentes estratégias: i) a subdivisão da geometria do modelo em subdomínios. Cada subdomínio é montado em um computador independentemente e trocam-se instruções entre os processadores para conectar as diferentes partes do mesmo problema. Esta técnica é descrita como decomposição em domínio com abordagem explícita; ii) a resolução do sistema é montada e resolvida em paralelo, sem levar em conta a geometria do problema físico. Esta estratégia é denominada de decomposição em domínio implícita.

A técnica da abordagem explícita é um procedimento que exige o uso de um algoritmo adequado para a divisão da geometria do problema físico de modo a garantir um equilibrado balanceamento de parâmetros dentro de cada subdomínio. O estudo desses algoritmos pode ser encontrado em SUN \& CHIEN (1994), WALSHAW et al. (1995), KARYPIS \& KUMAR (1995) e TOPPING \& KHAN (1996). 
Por outro lado, a aplicação da técnica da decomposição em domínio implícito não exige algoritmos de divisão de domínio, sendo que o principal enfoque dado para se explorar o paralelismo é no uso de um método de resolução de sistemas lineares que se ajuste a esta nova arquitetura.

Independentemente da técnica empregada para a montagem do problema, a resolução do sistema linear é o principal "gargalo" enfrentado para se obter mais alta performance em computação distribuída. Neste contexto, duas linhas de pesquisas focam o problema. Os grupos que aplicam os métodos diretos em processamento concorrente, onde podem-se citar os trabalhos de ZOIS (1988a e 1988b), ADELI \& KAMAL (1992), RAO et al. (1993), LAW \& MACKAY (1993), TOPPING \& KHAN (1996) e JIANG et al. (1997).

$\mathrm{E}$, por outro lado, os pesquisadores que empregam os métodos iterativos: CUMINATO \& MENEGUETTE (1998), GROSSO \& RIGHETTI (1988), LAW (1986), BITZARAKIS et al. (1997), BATHE (1982), DEMMEL (1993) e CIMERMAN (1996).

Para um melhor entendimento das vantagens e desvantagens da aplicação de um ou outro método de resolução de sistemas, ver ALMEIDA (1999).

\subsubsection{Decomposição em domínio com abordagem explícita}

A técnica da decomposição em domínio explícita pode ser descrita como dividir e conquistar, pois são algoritmos que objetivam a divisão do problema em uma série de subproblemas que são montados independentemente em cada computador e analisados em conjunto mediante os empregos das igualdades de fronteira entre as interfaces comuns dos subdomínios. Esta estratégia tem sido amplamente utilizada por muitos pesquisadores: FARHAT \& ROUX (1991), SAXENA \& PERUCCHIO (1992), BITZARAKIS et al. (1997) e ADELI \& KAMAL (1992).

Para se empregar esta técnica, é preciso destacar que as divisões dos diversos subdomínios não devem ser feitas aleatoriamente. Pois, como cada 
conjunto de subdomínios vai estar localizado em um determinado computador, nada impede que em cada um destes haja uma maior quantidade de graus de liberdade do que em outro processador, acarretando uma divisão da carga de trabalho desigual e, assim, o surgimento de tempo ocioso e comprometimento do desempenho do processamento distribuído.

Outro critério também importante para a melhor utilização da técnica de decomposição em domínio explícita é gerar o menor número possível de nós na interface, para que a comunicação entre os processadores seja mínima.

Assim, é comum o emprego das conhecidas técnicas de subestruturação (ver PRZEMIENIECKI (1962)). O procedimento para esta otimização consiste em fazer com que as variáveis internas de cada subdomínio sejam escritas em termos das incógnitas das interfaces de seu respectivo subdomínio. Resolve-se, então, o sistema particionado entre os processadores apenas com os parâmetros associados ao contorno, fazendo as trocas de mensagens necessárias entre os processos. Finalmente, para cada processador com posse dos deslocamentos das interfaces de seu respectivo subdomínio, calculam-se seus parâmetros internos.

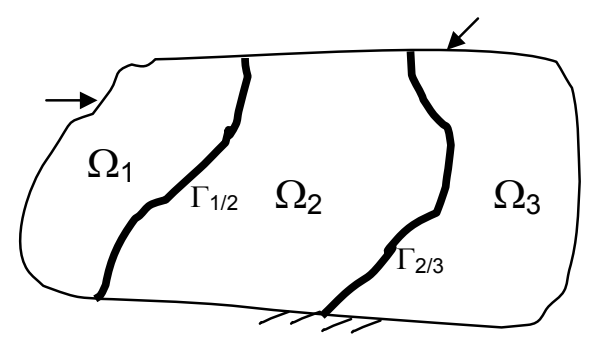

- Subdomínios: $\Omega_{1}, \Omega_{2}, \Omega_{3}$;

- Condensando valores para $\Gamma_{1 / 2}$ e $\Gamma_{2 / 3}$;

- Resolvendo problema de interface;

- Retornando em cada sub-região para cálculo seus de valores.

Figura 6.1 - Esquema simplificado da técnica da decomposição em domínio.

A subestruturação traz a vantagem de eliminar todos os graus de liberdade internos de cada subdomínio, o que diminui o número de variáveis para trocas entre os diversos processadores e, também, para se resolver o 
sistema linear, uma vez que a ordem de operações envolvidas nesta fase é $O\left(1 / 3 n^{3}\right)$, onde $n$ simboliza a dimensão da matriz final.

\subsubsection{Método da rigidez sucessiva aplicada em processamento distribuído}

A estratégia adotada neste trabalho é a da utilização da técnica da decomposição em domínio explícita com subestruturação. O procedimento apresentado no capítulo 3 para se avaliar a não-homogeneidade do solo enrijecido com elemento de fundação, chamado de "rigidez sucessiva", é visto como uma técnica de decomposição em domínio.

Cada estrato do solo representa um subdomínio, e pode-se construir suas matrizes de influência em cada processador, eliminando-se as sub-regiões que estão internas ao estrato - procedimento que cada processador realiza independentemente. Deste modo, as superfícies adjacentes "i" e "i+1" são equilibradas e compatibilizadas, e a matriz gerada na interface é armazenada no processador " $i+1$ ". Ver o esquema da figura (6.2), onde $P_{i}$ indica um processador genérico " $\mathrm{l}$ ”.

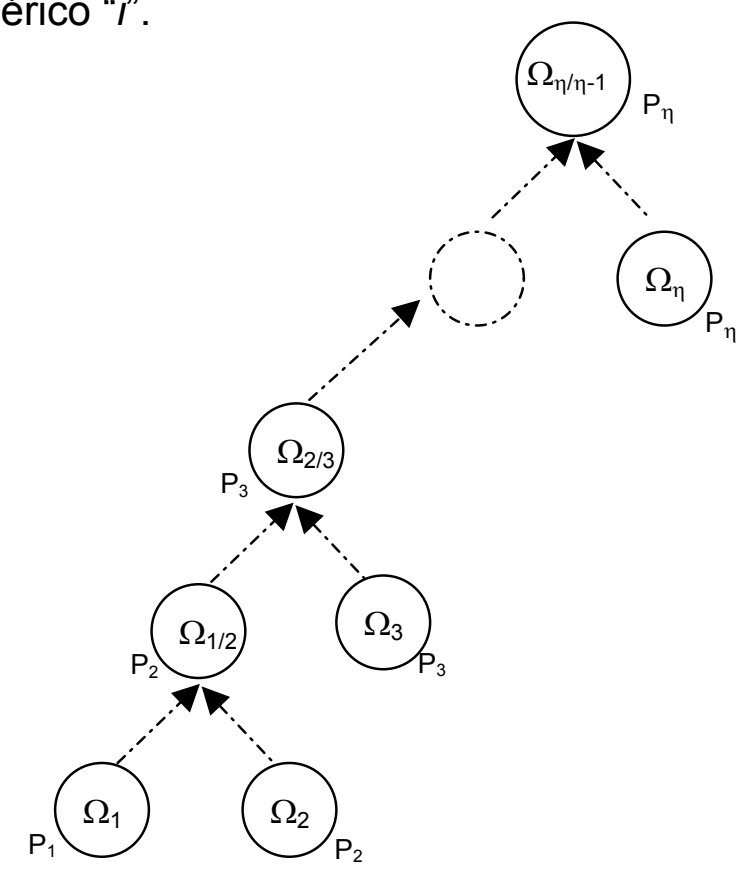

Figura 6.2 - Modelo de criação e transferência de dados em árvore entre processadores. 
A técnica da "rigidez sucessiva" apresenta vantagens na utilização de programas desenvolvidos tanto para processamento seqüencial quanto para processamento paralelo.

Pois esta técnica permite a simulação de problemas de grande escala sem a geração de sistemas que englobem todas as variáveis da geometria do modelo. Isso porque cada subdomínio é reescrito em função de seu domínio adjacente. E, no final, apenas as variáveis do contorno da superfície são empregadas para a resolução do sistema, diminuindo sensivelmente o número de operações para tal fase de análise.

Para o caso de processamento seqüencial, a decomposição em domínio com subestruturação é vantajosa, uma vez que se tem o limitante físico, que é a memória disponível para alocação das variáveis. E já foi mostrado que o número de operações envolvidas para se condensar as variáveis internas é menor que o número de operações envolvidos para a resolução do sistema linear (ver capítulo 3).

Em relação à aplicação dessa técnica em processamento distribuído, além das vantagens apresentadas em termos de operações, o ganho em termos de tempo é significativo, uma vez que os diversos subdomínios são montados simultaneamente.

No item (6.4), são apresentados alguns exemplos onde se mostram seus parâmetros de desempenho.

\subsubsection{Medida de desempenho}

A medida usual de desempenho no processamento de programas ou de trechos paralelos é o fator conhecido como Speed-up. Este equivale à relação do quociente entre o tempo necessário para sua execução em programação sequencial e o tempo em processamento paralelo com $n$ processadores.

Assim, esta relação é expressa por:

$$
\text { Speed }- \text { up }=\frac{\text { Tempo com } 1 \text { processador }}{\text { Tempo com } \mathrm{n} \text { processadores }}
$$


Esta relação mostra que para valores acima de um, tem-se um ganho na execução do processamento com $n$ processadores. Aliada à medida de speedup, tem-se a medida de eficiência do processo, a qual é dada pela relação:

$$
\text { Eficiência }=\frac{100 \cdot(\text { Speed }- \text { up })}{\text { número de processadores }}(\%)
$$

Em AMDAHL (1967), mostra-se que o limite superior do speed-up é dado pela relação

$$
\text { Speed }-u p \leq \frac{1}{s+\frac{r}{n}}
$$

de tal modo que $r$ representa a fração em tempo do processo que é paralelizável dentro programa, e s é a fração que deve ser executada seqüencialmente, ou seja, $s=r-1$.

Assim, por exemplo, onde o tempo de análise em paralelo é bem superior que o tempo sequencial, pode-se, sem perda de generalidade, afirmar que $r=1$, assim, cem por cento do programa é paralelizável. Portanto, nestes casos, o speed-up é limitado por:

$$
(\text { Speed }-u p) \leq n
$$

O speed-up, como já salientado, é um medidor de desempenho do processamento que compara a performance do programa seqüencial com sua versão em paralelo. Entretanto, conforme mostrado pela equação (6.4), simplificadamente é estabelecido que seu valor está limitado pelo número de processadores. E, em geral, o valor do speed-up ficará bem abaixo deste valor, podendo até ser um valor menor que um, para um número reduzido de processadores.

Isto ocorre, principalmente, em virtude do "gargalo" surgido quando da necessidade de espera dos processadores para trocas de mensagens e da 
sincronização dos processadores dentro dos processos e/ou das instruções, principalmente para o caso aqui tratado, onde se têm poucos processadores para verificar os fatores de desempenho.

Para destacar esta perda de eficiência do processamento paralelo, em JIANG et al. (1997) obtém-se um speed-up em torno de 10 para a análise em paralelo com 22 computadores - cada um com sua memória local - de um sistema com a fração em paralelo bem próximo a um. A eficiência obtida em seu trabalho foi de $45 \%$.

\subsubsection{Modelo mestre-escravo do PVM}

O sistema Parallel Virtual Machine (PVM) é um pacote empregado dentro dos códigos convencionais, e tem 0 intuito de interligar os diversos computadores para realizarem entre si a comunicação entre os diversos dados envolvidos na resolução de um problema em paralelo. O PVM usa, então, o modelo de passagem de mensagem para permitir programadores explorarem a computação distribuída através de uma extensa variedade de tipos de computadores. O conceito chave no PVM é que ele faz com que uma coleção de computadores pareça como uma grande máquina virtual, por isso o seu nome.

O paradigma mais comumente empregado pelo PVM é o modelo mestreescravo, o qual possui um programa principal, nomeado de mestre, responsável pela geração (spawning) do processo, inicialização das instruções em paralelo, obtenção dos resultados dos outros processadores e pela saída destes em arquivos. Enfim, ele é responsável pelo gerenciamento das tarefas que serão executadas pelos diferentes processadores. Os programas escravos executam a computação necessária, sendo que eles são gerados e controlados pelo processo mestre.

Toda esta computação envolve 3 fases: 
- A primeira é a inicialização do processo no grupo, ou seja, no programa onde se aplica as subrotinas do PVM. O mestre aloca o número de processos escravos requeridos pelo usuário e coloca todos (mestre mais escravos) em um grupo.

- A Segunda fase é a computação em si, onde todos do grupo executam as tarefas atribuídas a cada um deles e, quando necessário, trocam, transmitem ou recebem dados de outros processos, sendo que esta comunicação pode ser feita entre todos do grupo.

- A terceira fase é a obtenção e a apresentação dos resultados e, finalmente, o grupo participante de todo o processo é desfeito, terminando assim o processamento em paralelo. Podendo, ainda, ser executado instruções pelo processador mestre, em série e, se necessário, iniciar a geração novamente com um novo grupo.

O sistema PVM foi desenvolvido para implementação em programas que sustentam as seguintes linguagens: $\mathrm{C}, \mathrm{C}++$ e FORTRAN. As subrotinas existentes para essas linguagens somam mais de 100, e que conseguem atender aos problemas convencionais do usuário. Caso seja necessário, podem ser obtidos tanto o sistema PVM com subrotinas em C e em FORTRAN, como o seu manual contendo todas as subrotinas, via ftp pela rede, mediante o seguinte endereço: netlib2.cs.utk.edu; cd pvm3/book; get refcard.ps.

\subsubsection{Exemplos numéricos}

Nesse sentido, o código computacional desenvolvido neste trabalho foi implementado tanto em processamento sequencial, como em processamento paralelo usando o sistema de passagem de mensagem PVM.

Apresenta-se, então, um exemplo numérico avaliado em processamento paralelo, onde o resultados são obtidos na máquina do CISC localizada no 
campus da USP em São Carlos, sendo um computador com arquitetura paralela do tipo IBM 9900/SP com 3 processadores com 256 Mbytes por nó, ver TUTORIAL do IBM (1998) e TUTORIAIS do CISC (1998).

\subsubsection{Whitaker sem estaca}

Este exemplo é constituído por uma rede formada por 90 nós e 144 elementos para cada camada do solo, e representa o problema apresentado no item 2.7.4, figura 2.19, mas já empregando a técnica mostrada no capítulo $3 \mathrm{e}$ sem a consideração do elemento de fundação.

Dividiram-se as camadas do maciço de modo a se ter cada processador processando sobre cada estrato. Assim, as tabelas 6.1 a 6.4 avaliam o speedup e a eficiência obtidos para esses diversos casos. Todos os tempos são medidos em segundo.

Tabela 6.1:

Para 2 camadas do solo.

\begin{tabular}{|c|c|c|c|c|c|}
\hline $\mathrm{N}^{\circ}$ de processadores & $\mathrm{T}_{\text {montagem }}$ & $\mathrm{T}_{\text {resolução }}$ & $\mathrm{T}_{\text {total }}$ & Speed-up & Eficiência (\%) \\
\hline 1 & 241,99 & 0,031 & 242,02 & 1 & $\mathbf{1 0 0}$ \\
\hline 2 & 123,22 & 0,034 & 123,27 & 1,96 & $\mathbf{9 8 , 2}$ \\
\hline 1 Plataforma windows & 309,67 & 0,05 & 309,72 & - & - \\
\hline
\end{tabular}

Tabela 6.2:

Para 3 camadas do solo.

\begin{tabular}{|c|c|c|c|c|c|}
\hline $\mathrm{N}^{\circ}$ de processadores & $\mathrm{T}_{\text {montagem }}$ & $\mathrm{T}_{\text {resolução }}$ & $\mathrm{T}_{\text {total }}$ & Speed-up & Eficiência (\%) \\
\hline 1 & 367,89 & 0,033 & 367,92 & 1 & $\mathbf{1 0 0}$ \\
\hline 2 & 242,16 & 0,033 & 242,19 & 1,52 & $\mathbf{7 6}$ \\
\hline 3 & 124,08 & 0,031 & 124,11 & 2,96 & $\mathbf{9 8 , 8}$ \\
\hline
\end{tabular}

Tabela 6.3:

Para 4 camadas do solo. 


\begin{tabular}{|c|c|c|c|c|c|}
\hline $\mathrm{N}^{\circ}$ de processadores & $\mathrm{T}_{\text {montagem }}$ & $\mathrm{T}_{\text {resolução }}$ & $\mathrm{T}_{\text {total }}$ & Speed-up & Eficiência (\%) \\
\hline 1 & 484,38 & 0,031 & 484,41 & 1 & $\mathbf{1 0 0}$ \\
\hline 2 & 244,98 & 0,033 & 245,81 & 1,97 & $\mathbf{9 8 , 5}$ \\
\hline 3 & 245,54 & 0,032 & 245,57 & 1,97 & $\mathbf{6 5 , 7}$ \\
\hline
\end{tabular}

Tabela 6.4:

Para 6 camadas do solo.

\begin{tabular}{|c|c|c|c|c|c|}
\hline $\mathrm{N}^{\circ}$ de processadores & $\mathrm{T}_{\text {montagem }}$ & $\mathrm{T}_{\text {resolução }}$ & $\mathrm{T}_{\text {total }}$ & Speed-up & Eficiência (\%) \\
\hline 1 & 726,45 & 0,032 & 726,49 & 1 & $\mathbf{1 0 0}$ \\
\hline 2 & 368,54 & 0,033 & 368,57 & 1,97 & $\mathbf{9 8 , 6}$ \\
\hline 3 & 246,52 & 0,032 & 246,55 & 2,95 & $\mathbf{9 8 , 2}$ \\
\hline
\end{tabular}

\subsubsection{Lâmina quadrada apoiada sobre meio semi-infinito}

Este exemplo representa o problema analisado no item (5.2), com o uso da rede a), vide figura (5.2), o qual é constituído por uma rede formado por 454 nós e 744 elementos para cada camada do solo. Deste modo, dividiu-se o meio em 3 camadas, apresentando então os resultados de tempo processamento quando se emprega 1 e 3 processadores.

Tabela 6.5:

Para 3 camadas do maciço.

\begin{tabular}{|c|c|c|c|c|c|}
\hline $\mathrm{N}^{\circ}$ de processadores & $\mathrm{T}_{\text {montagem }}$ & $\mathrm{T}_{\text {resolução }}$ & $\mathrm{T}_{\text {total }}$ & Speed-up & Eficiência (\%) \\
\hline 1 & 22828,70 & 72,25 & 22900,95 & 1 & $\mathbf{1 0 0}$ \\
\hline 3 & 8049,04 & 61,20 & 8110,26 & 2,82 & $\mathbf{9 4 , 1 2}$ \\
\hline
\end{tabular}

\subsubsection{Uma estaca no meio finito não-homogêneo}

Avalia-se o tempo de processamento paralelo para o exemplo apresentado no capítulo 5 , item 5.8, o qual representa uma estaca imersa em um meio finito não-homogêneo. Assim, o maciço é constituído de 3 camadas, onde cada camada possui 554 nós e 1056 elementos, onde também deve se 
somar para a primeira e segunda camada o número de nós gerados pela estaca, assim, estes dois estratos possuem um total de 680 nós. Apresenta-se na tabela 6.6 o speed-up e a eficiência para a o caso de se empregar 3 processadores.

Tabela 6.6:

Para 3 camadas do maciço.

\begin{tabular}{|c|c|c|c|c|c|}
\hline $\mathrm{N}^{\circ}$ de processadores & $\mathrm{T}_{\text {montagem }}$ & $\mathrm{T}_{\text {resolução }}$ & $\mathrm{T}_{\text {total }}$ & Speed-up & Eficiência (\%) \\
\hline 1 & 46191,74 & 156,46 & 46348,20 & 1 & $\mathbf{1 0 0}$ \\
\hline 3 & 18237,24 & 156,33 & 18393,57 & 2,52 & $\mathbf{8 3 , 9 9}$ \\
\hline
\end{tabular}

Os três exemplos apresentados mostraram que o uso da técnica da decomposição em domínio empregada, considerando cada camada como se fosse um domínio, demonstrou ser uma maneira eficiente para se obter boa performance do processamento. É válido se acrescentar que a implementação do código sequencial para processamento paralelo foi imediata, sem complicados expedientes adicionais, sendo isto o ponto chave do emprego do paralelismo, ou seja, utilizá-lo apenas como uma ferramenta numérica, sem a necessidade de profundas alterações no código sequencial.

\subsection{Resolução de sistemas lineares esparsos}

Nos problemas convencionais de análise numérica, como na resolução de sistemas lineares, nas técnicas de mínimos quadrados e nos autoproblemas, é imprescindível a exploração de qualquer estrutura especial existente no problema.

Por exemplo, na resolução de um sistema linear de dimensão $n x n$, o custo computacional é da ordem de $2 / 3 n^{3}$ operações em ponto flutuante no caso de se usar a mais geral forma da eliminação de Gauss. Se for somado ao 
problema a informação de que o sistema é simétrico e positivo definido, podese economizar metade do trabalho, onde se chega a um outro algoritmo denominado de Cholesky. Caso se adicione ainda ao problema a característica de banda à matriz, com semicomprimento de banda de dimensão $m$ (ou seja $a_{i j}=0$ se $\left.|i-j|>m\right)$, então pode-se reduzir o custo a ordem de $1 / 2 n \cdot m^{2}$ usando-se o procedimento chamado de Cholesky em banda.

Para o presente trabalho, a matriz final do acoplamento entre o solo e a superestrutura pode gerar uma forma esparsa, onde a definição de esparsidade esta relacionada ao fato de que os coeficientes nulos da matriz são em maior quantidade que os não-nulos. Essa esparsidade acontece se existir regiões em que o solo e a superestrutura não se coincidem, por exemplo, para o caso de um edifício sobre o solo. Além disso, no acoplamento MEC/MEF a matriz final gerada não é nem simétrica, e nem possui, a priori, uma estrutura de dados que se configura em banda. É necessário, então, procurar algoritmos de resolução de sistemas que tratem este tipo peculiar de matriz.

As técnicas de resolução de sistemas lineares se dividem em duas grandes frentes distintas: os métodos diretos, e os métodos iterativos. Para o caso da aplicação dos métodos diretos sobre matrizes esparsas, citam-se os trabalhos de DUFF \& REID (1982) e DEMMEL (1993) para sistemas simétricos, e DUFF (1977) para não-simétricos, e DUFF et al. (1990) é uma excelente referência para estudar os métodos diretos para matrizes esparsas. Para a aplicação via métodos iterativos, têm-se os trabalhos de ALMEIDA \& PAIVA (2000), ALMEIDA \& PAIVA (1999), CIMERMAN (1996) e SCHMIT \& LAI (1994) para as matrizes simétricas, e para as não-simétricas se tem o trabalho pioneiro de SAAD \& SCHULTZ (1986).

A maior vantagem que existe para o emprego dos métodos diretos em sistemas esparsos é a respeito de que esses métodos não são sensíveis ao condicionamento da matriz quanto à convergência do sistema. Assim, o número de operações em ponto flutuante necessário para se obter o vetor solução é 
conhecido a priori, independente do número de condição da matriz, desde que este não seja infinitamente grande, o que representa uma matriz singular.

Como maior desvantagem do método aplicado a problemas esparsos é em relação ao efeito de fill-in, ou termo também denominado de efeito de preenchimento, que pode prejudicar a convergência do algoritmo. Isso acontece porque, em geral, os métodos diretos se baseiam no processo de eliminação de Gauss, e sabe-se que quando a matriz esparsa está sendo fatorizada, dependendo da posição dos valores não-nulos, as matrizes triangulares se tornam não-esparsas, o que acarreta em uma geração e posterior manipulação de dados extra. Para o caso da matriz apresentar uma típica estrutura em banda, o efeito de fill-in não ocorre, o que evidencia o uso dos métodos diretos.

Em relação ao uso dos métodos iterativos, a maior vantagem existente para o seu emprego é que estes métodos independem da estrutura de dados existentes na matriz, ou seja, não há efeito de fill-in, uma vez que estes métodos não alteram a matriz do sistema, mas realizam iterativamente o produto matriz-vetor, e averiguam a norma entre este vetor resultante e o vetor independente, e a direção tomada para o próximo vetor resposta depende do método iterativo empregado.

Como maior desvantagem para o uso dos métodos iterativos, é em relação a sensível dependência de convergência com relação ao condicionamento da matriz, uma vez que as direções tomadas a cada iteração podem não levar a um campo mais próximo da resposta desejada, já que essas direções obtidas - a cada iteração - dependem de uma relação espectral da matriz, ou seja, do seu condicionamento. Isso, na prática, representa que para um sistema bem-condicionado, ou seja, número de condição próximo do valor unitário, a convergência pode ocorrer em poucas iterações, quase que independente do vetor resposta inicial adotado. Por outro lado, para sistemas mal-condicionados, essa convergência é sofrível, quando não impossível, mesmo usando-se técnicas de aceleração de convergência, denominadas de técnicas de pré-condicionamento. 
Em suma, escolher o melhor método para resolução de sistemas esparsos depende fortemente de dois critérios: i) conhecer a estrutura de dados da matriz; ii) conhecer o número de condição do sistema. Assim, a busca do melhor método vai depender da análise desses critérios.

\subsubsection{Aplicações numéricas}

Apresenta-se neste item três exemplos que avaliam a influência da esparsidade, da estrutura de dados e do número de condição perante o uso de alguns métodos de resolução de sistemas lineares. Os exemplos foram executados em computadores pessoais e em computação sequencial.

\subsubsection{Acoplamento edifício/radier/solo}

Avaliou-se o tempo de processamento apenas para a resolução do sistema linear gerado no exemplo do acoplamento edifício/radier/solo, item 5.5. A matriz final contém 4431 equações, onde a quantidade de coeficientes nãonulos é de $26 \%$ (5,1E6 coeficientes). Na figura 6.3, pode-se notar a estrutura de dados existente para este caso.

No trecho inicial da matriz, percebe-se que os coeficientes não-nulos estão concentrados ao redor da diagonal principal, este trecho representa os termos de influência do edifício via MEF. O segundo trecho representa a região de contato entre os elementos finitos e os elementos de contorno, e as regiões em branco indicam a inexistência de influência entre os parâmetros de rotação da lâmina com os do solo, pois para o solo as rotações não existem. O terceiro trecho indica a região de influência entre os valores apenas do solo, assim essa região representa uma submatriz populosa, uma vez que essa é a característica das matrizes do MEC.

Tem-se implementado no código computacional três métodos de resolução de sistemas esparsos não-simétricos: 
a) Método direto baseado nas rotinas da biblioteca IMSL, desenvolvido por Aird et al. (1977), mas que não aproveita a característica esparsa da matriz;

b) Método direto baseado no pacote de matrizes esparsas, desenvolvido no laboratório de Harwell na Inglaterra por DUFF (1977), onde a versão empregada é a família do MA28;

c) Método iterativo denominado de GMRES (sigla de Generalized Minimum Residual), originalmente proposto por SAAD \& SCHULTZ (1986), sendo que nesse exemplo usou-se o précondicionador Jacobi.

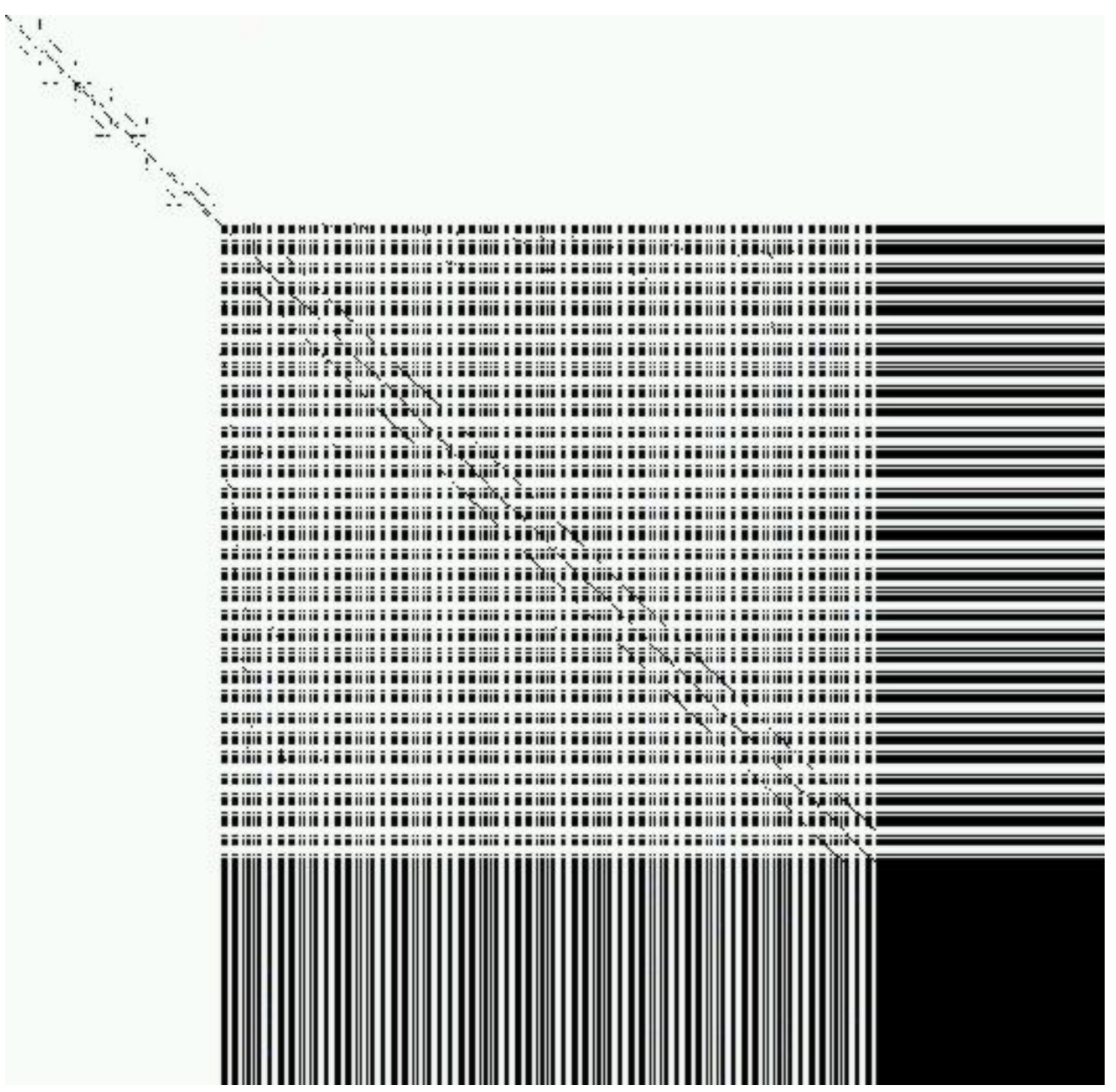

Figura 6.3 - Estrutura de dados para a matriz gerada no acoplamento edifício/radier/solo. 
Tabela 6.7:

Tempo (minutos) para resolução do sistema linear com 4431 graus de liberdade e com $26 \%$ de coeficientes não-nulos.

\begin{tabular}{ccc}
\hline $\begin{array}{c}\text { Métodos para resolução de } \\
\text { equações lineares }\end{array}$ & $\begin{array}{c}\text { Pentium III, 1 GHz processador, } \\
\text { 1GB PC133de memória Ram }\end{array}$ & $\begin{array}{c}\text { Pentium IV Dual, } \\
\text { 1.7GHz, } 2 \text { GB RDRAM }\end{array}$ \\
\hline Rotinas do IMSL - DLSLRG & 220 & 164,1 \\
GMRES: $(\varepsilon=1 \mathrm{e}-9)$ & 46,6 & 25,4 \\
GMRES: $(\varepsilon=1 \mathrm{e}-6)$ & 22,4 & 15,2 \\
MA28 & 19,0 & 11,9 \\
\hline
\end{tabular}

A primeira técnica, a qual é baseada no método direto, não leva em conta a propriedade de esparsidade da matriz final, assim ele não é adequado para resolução deste tipo de problema, como pode ser verificado seu tempo de resolução na tabela 6.7 .

O segundo, é o mais convencional método iterativo aplicado para a solução de sistemas lineares não-simétricos esparsos. O custo computacional do método, como já salientado, é para a realização de um produto matriz-vetor ao longo de cada iteração, e a configuração esparsa gerada no final da matriz não representa um fator relevante para a convergência do método. Mas, a maior dependência do GMRES é devido ao número de condição da matriz, e para a aplicação em problemas elastostásticos do MEC, em geral, essa propriedade da matriz não é bem-condicionada, principalmente, para o caso de acoplamentos entre MEC/MEC e MEC/MEF.

O pacote de matrizes esparsas do laboratório Harwell, MA28, o qual é baseado no processo de eliminação de Gauss, mostra que a estrutura de dados gerada na matriz, figura 6.3, é um fator determinante para uma rápida execução do código, isso devido ao efeito de fill-in. Entretanto, nenhum tipo de técnica ou software existentes para otimizar a estrutura de dados foi empregado, e mesmo assim o tempo para resolução do problema proposto neste item, vide tabela 6.5 , 
mostra uma boa eficiência na resolução de problemas advindos do acoplamento MEC/MEF.

\subsubsection{Problema MEC/MEC para o caso bidimensional}

Este problema é baseado na formulação do MEC utilizando a solução de Kelvin para o caso bidimensional. Avalia-se, então, um problema onde se consideram quatro sub-regiões (vide figura 6.4). Na figura 6.5 apresenta-se a configuração da estrutura de dados gerada para a matriz final do sistema linear.

A tabela 6.8 compara as diferentes técnicas de resolução de sistemas. O método direto MA28 não apresentou vantagem, isso porque o método é dividido em dois grandes estágios: i) o estágio de ANÁLISE da estrutura de dados; ii) o estágio de FATORAÇÃO dos dados. No primeiro estágio, se escolhe os coeficientes que serão os pivôs e se identifica a estrutura de fill-in da matriz. Essa primeira parte exige em torno de $70 \%$ do tempo total do método. E como a matriz esparsa final possui uma estrutura de dados que leva a geração de uma matriz densa na fatorização, isso devido ao grande efeito de fill-in, este estágio se torna o grande gargalo do uso do MA28, e quanto maior o efeito fill-in tem na matriz, mais inadequado é seu uso para a resolução de sistemas esparsos.

O método iterativo não depende dessa configuração de dados, só é dependente do condicionamento da matriz final. É necessário comentar que todas as sub-regiões apresentam as mesmas propriedades físicas e geométricas, assim, as gerações das matrizes de influência $H$ e $G$ são, independentemente, bem-condicionadas. Entretanto, a matriz final do sistema, onde se misturam as matrizes $\mathrm{H} \mathrm{e} \mathrm{G}$, em virtude da característica intrínseca do MEC, leva a uma matriz final não tão bem-condicionada. A rotina do IMSL empregada não leva em consideração a característica esparsa da matriz, e o tempo de resolução usando esta rotina, tabela (6.8), demonstram sua baixa eficiência para casos esparsos. 
Tabela 6.8:

Tempo (segundos) para resolução do sistema linear com 1312 graus de liberdade e com 33\% de coeficientes não-nulos.

\begin{tabular}{cc}
\hline $\begin{array}{c}\text { Métodos para resolução } \\
\text { de equações lineares }\end{array}$ & $\begin{array}{c}\text { Pentium III, } 1 \mathrm{GHz} \\
\text { processador, 1GB } \\
\text { PC133de memória Ram }\end{array}$ \\
\hline Rotinas do IMSL - DLSLRG & 189,85 \\
GMRES: $(\varepsilon=1 \mathrm{e}-14)$ & 25,52 \\
GMRES: $(\varepsilon=1 \mathrm{e}-9)$ & 12,20 \\
GMRES: $(\varepsilon=1 \mathrm{e}-6)$ & 9,51 \\
MA28 & 36,67 \\
\hline
\end{tabular}

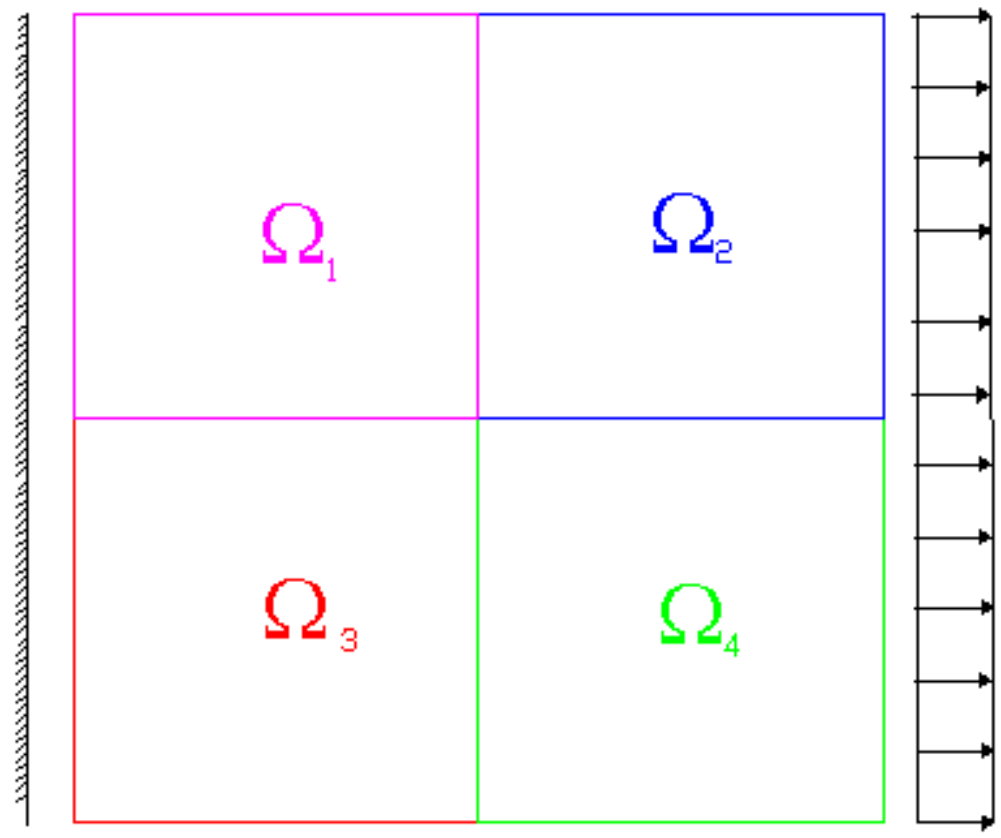

Figura 6.4 - Estrutura de dados para a matriz gerada em um problema MEC/MEC 2D. 


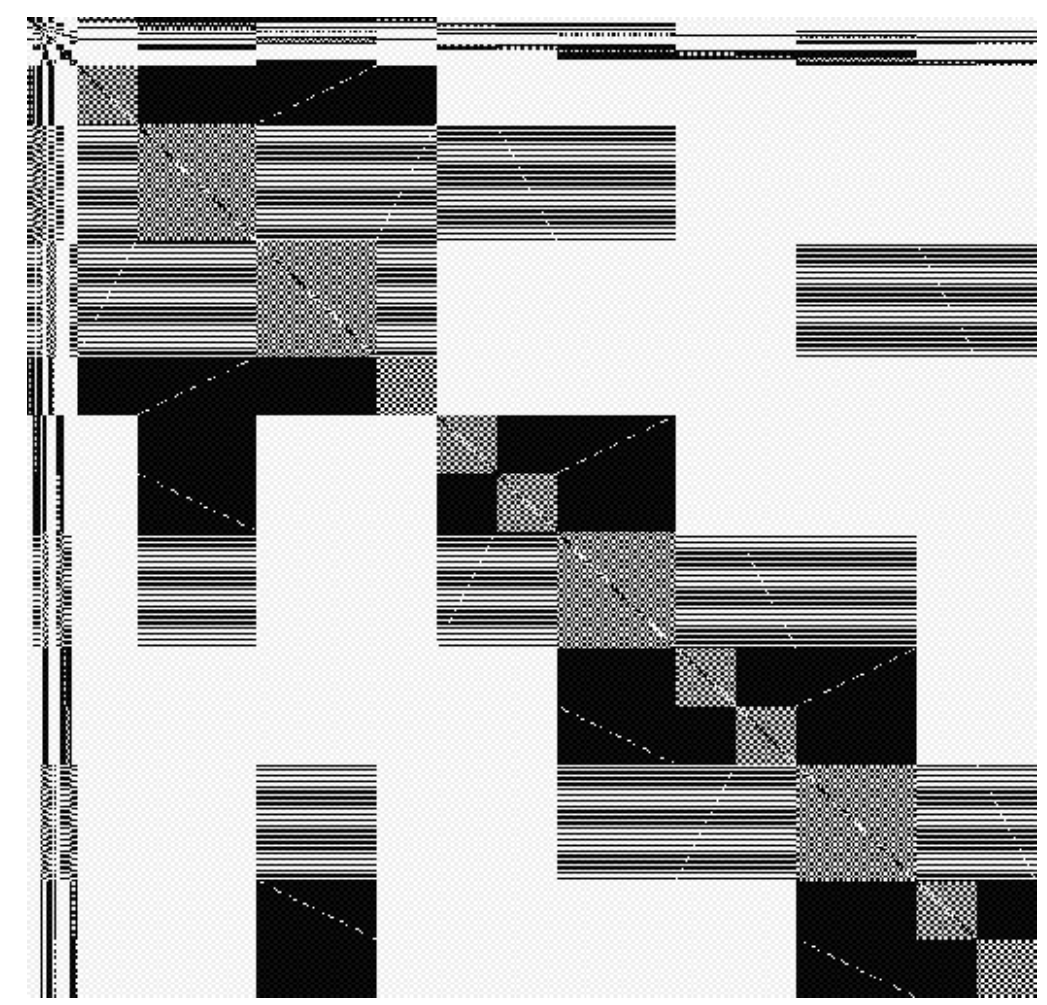

Figura 6.5 - Estrutura de dados para a matriz gerada em um problema MEC/MEC 2D.

\subsubsection{Problema da membrana de Cook via MEF}

O exemplo tem o intuito de mostrar diferentes modelos de resolução de sistema aplicados para os métodos dos elementos finitos. Como as matrizes do MEF são simétricas e esparsas, pode-se utilizar métodos de resolução que explorem estas características.

Assim, comparou-se o método iterativo denominado de Gradientes Conjugados com e sem a aplicação de pré-condicionadores com dois métodos diretos.

O método dos Gradientes Conjugados e os pré-condicionadores empregados podem ser estudados no trabalho de ALMEIDA \& PAIVA (1999) e ALMEIDA \& PAIVA (2000).

O primeiro método direto empregado é o método de eliminação de Gauss convencional sem que se aproveite a esparsidade intrínseca da matriz. Assim 
ela é armazenada em uma matriz retangular de dimensão de número de linhas igual ao total de graus de liberdade e de colunas igual ao comprimento de banda.

O segundo método é o MA27 que é um método direto baseado em uma variante esparsa do método de eliminação de Gauss que é discutido em DUFF \& REID (1982).

Neste sentido, foram avaliados estes diversos métodos em um exemplo bem conhecido que é a membrana de Cook, BERGAN \& FELIPPA (1985). Geraram-se então várias redes aumentando-se a quantidade de elementos finitos, e a quantidade de graus de liberdade, para verificar o comportamento dos métodos de resolução frente a estas variações. As malhas geradas mantiveram em média um índice de esparsidade de $95 \%$, ou seja, em torno de $5 \%$ dos coeficientes da matriz eram diferentes de zero.

Para as matrizes geradas foram calculados seus respectivos números de condição. Estes parâmetros indicam o nível de condicionamento das matrizes, pois os métodos iterativos são dependentes destes valores. Assim, para estes exemplos em análise, os números de condição encontrados indicam que as matrizes estão mal-condicionadas.

Entretanto, para os tempos encontrados, o pacote MA27 demonstrou ser muito mais eficiente que os outros métodos aplicados.

Comparando-o com o segundo melhor método empregado, o Gradiente conjugado (CG) sem pré-condicionador a diferença de tempo foi de $80 \%$, sendo que MA27 demorou 30,9 segundos e o CG 159,0 segundos. 
Figura 6.6 - Estrutura plana com carga unitária uniformemente distribuída ao longo do contorno da direita com $E=1, v=1 / 3$.

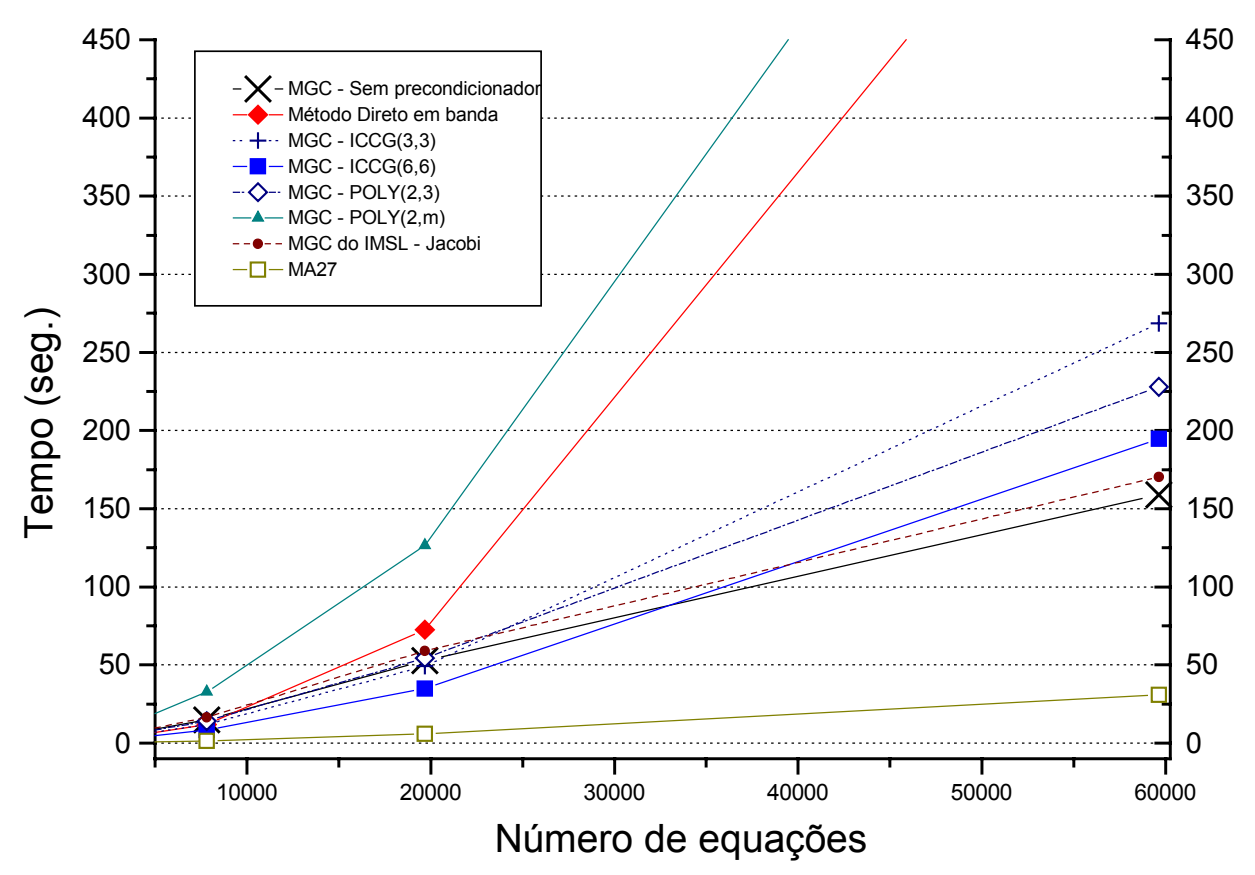

Figura 6.7 - Tempo de resolução para os diferentes métodos de resolução. 
Os três últimos sistemas lineares gerados foram concebidos por formulações distintas:

1. o primeiro foi resultado do acoplamento MEC 3D/MEF 2D/MEF barras lineares;

2. o segundo resultou do MEC 2D para várias sub-regiões;

3. o terceiro foi originado do MEF 2D;

Cada um possui suas características peculiares para a geração do sistema final, que influencia no condicionamento e na estrutura de dados da matriz.

Esses exemplos demonstraram que essas duas características são de suma importância para a performance de um ou de outro método de resolução de sistemas esparsos.

Como conclusão, deve-se antes de escolher um método de resolução, tomar conhecimento principalmente com relação a estrutura de dados da matriz final. Pois, caso a matriz possua uma estrutura de dados com forma bem regular, ou seja, sem aumentar o efeito fill-in, é válido empregar as técnicas diretas que aproveitem as propriedades esparsas, por exemplo, usando-se os pacotes da família MA27 ou MA28, como pode ser visto nos exemplos dos itens (6.3.1.1) e (6.3.1.3).

Mas, para o caso da estrutura de dados da matriz final levar ao aumento do efeito fill-in, item (6.3.1.2), pode-se aplicar os métodos iterativos sem ou com precondicionamento. Entretanto, tendo-se em mente que para problemas iterativos, em função necessidade de grandes precisões das respostas em cada iteração se é necessário escolher um critério de convergência muito pequeno, o que aumenta muito o número de iterações para convergência do método. Isto torna o método impróprio ou não competitivo com os métodos diretos. Para minimizar a influência do efeito fill-in fora da região de banda, item (6.3.1.2), é comum se empregar ferramentas que manipule a matriz final do sistema de 
maneira a reordenar as posições destas posições para que tornem essa matriz fatorada também esparsa, sem perder a precisão das respostas.

Por fim, é sempre importante empregar um método de resolução de sistema esparsos que aproveite essas características especiais da matriz, pois assim não se necessita de armazenar os coeficientes não-nulos. 


\section{Capítulo 7}

\section{CONSIDERAÇÕES FINAIS}

O objetivo do trabalho é a análise do complexo sistema que envolve o acoplamento entre 0 maciço de solos/subestrutura/infraestrutura/superestrutura. Foi apresentado então o histórico bibliográfico existente para simular as diversas formulações deste sistema. É lícito confirmar que a simulação deste especial problema da engenharia civil deve ser avaliado via o emprego dos métodos numéricos. Com o grande desenvolvimento dos diversos modelos mecânicos e geométricos que podem ser encontrados na literatura corrente, e que são implementados via MEC ou MEF, é fácil incorporar esses fenômenos nos elementos estruturais do sistema em estudo, ficando evidente a aplicação dessas poderosas ferramentas nesta análise.

Neste sentido, o projeto foi desenvolvido para a avaliação deste conjunto mecânico, e pelos resultados encontrados o objetivo foi plenamente atingido.

O maciço de solos foi considerado mediante o emprego do método dos elementos de contorno tridimensional. Este é o método mais adequado para analisar este tipo de problema, pois, no MEC as funções ponderadoras já contemplam as restrições a grandes distâncias; e as variáveis do problema só estão associadas a valores de contorno para as hipóteses elastostáticas do modelo. Este último item leva à economia na geração e na manipulação dos dados do maciço, onde esta vantagem se torna mais evidente para o caso presente em que se utiliza diversas sub-regiões para simular o meio heterogêneo. 
A subestrutura, que pode simular os elementos estruturais de estacas, tubulões e sapatas, também foi representada via MEC 3D. As respostas obtidas para a análise do conjunto solo/subestrutura demonstraram resultados satisfatórios, afirmando-se que o acoplamento MEC/MEC 3D é uma boa alternativa para a representação desse tipo de problema. Enfatizase que a vantagem existente para este tipo de acoplamento deve se sobressair para a análise de problemas em que se tenham as condições de fronteira de Dirichlet, como, por exemplo, para a análise de problemas de contato e/ou para a análise inversa.

A infra e a superestrutura foram formuladas empregando-se o método dos elementos finitos, que é a melhor ferramenta para a representação deste tipo de elementos estruturais. Usou-se, então, o bem conhecido elemento finito de geometria triangular plana, o qual considera o efeito de membrana e de placa fina de Kirchhoff. A simulação do edifício foi realizada via o emprego do MEF com elementos reticulados que não consideram o efeito de torção.

Com essas ferramentas numéricas, pode-se analisar cada um dos sistemas estruturais isolados, ou considerá-los de maneira integrada dentro do mesmo pacote computacional. Assim, no quinto capítulo são apresentados alguns exemplos numéricos que foram extraídos na literatura indicada. Os resultados encontrados demonstraram uma boa concordância com os trabalhos de comparações. Alguns outros exemplos foram apresentados sem compará-los com outras formulações, em virtude da escassa existência de referência. Por exemplo, na análise do edifício apoiado em radier em meio não-homogêneo, ou na avaliação das estacas imersas em meio heterogêneo. Entretanto, todas as possibilidades de análise isolada da presente formulação, ou seja, solo homogêneo ou heterogêneo apoiado em camada incompressível, solo homogêneo ou heterogêneo/radier, solo homogêneo/estaca sobre camada incompressível, foram comprovadas mediante comparações com exemplos analíticos e/ou numéricos. 
É fato que os dois grandes "gargalos" existentes na resolução de um problema via o uso dos métodos numéricos são referentes à: i) montagem do sistema final de equações; ii) resolução do sistema de equações gerado.

Estes dois pontos débeis se tornam mais cruciais quando se emprega o método dos elementos de contorno, pois este método leva a montagem de uma matriz final totalmente povoada, o que culmina em uma maior quantidade de operações necessárias para montar o sistema final e sua posterior resolução.

Neste sentido, objetivando-se um menor tempo para resolução do problema, o presente projeto também empregou duas importantes ferramentas numéricas para atenuar esses problemas: o uso do paralelismo na montagem das matrizes densas do MEC, e o emprego de uma adequada técnica de resolução de sistemas esparsos gerados no acoplamento MEC/MEF.

O paralelismo é uma ferramenta que tem sido amplamente empregada no campo dos métodos numéricos, pois representa um expediente natural para a busca de melhores eficiências em termos de tempo. Entretanto, utilizar o paralelismo de maneira explícita, ou seja, onde o programador tenha que empregar esta filosofia em seu próprio código, leva a profundas mudanças nos procedimentos convencionais já consagrados nos MEC e/ou MEF, onde se pode levar muito tempo para realizar estas alterações.

Optou-se, então, por usar a computação distribuída apenas nos pontos de montagem das matrizes de influência de cada sub-região do solo. Isto foi feito mediante o emprego da técnica da decomposição em domínio, onde se atribuiu a cada processador a tarefa de montar as suas matrizes referentes a seu respectivo estrato de referência e, ao fim, associar sua influência no sistema final. Assim, as tarefas de cada estrato são realizadas simultaneamente e a manutenção de independência entre os diversos processos é atendida naturalmente. Esta idéia mostrou-se bastante eficiente, uma vez que os resultados apresentados no capítulo seis demonstraram que 
os valores de eficiência para as diversas combinações de processadores foram superiores a $90 \%$.

O emprego de um método de resolução de sistemas que aproveite as características esparsas da matriz final do sistema gerado no acoplamento MEC/MEF mostrou ser uma outra grande questão a ser considerada para se resolver o problema de maneira mais rápida. Os exemplos apresentados no final do capítulo seis foram suficientes para demonstrar essa necessidade, uma vez que o uso do adequado método que aproveite essas características especiais da matriz leva a uma economia significativa de espaço para o seu armazenamento e de tempo. Principalmente para as aplicações em que se usam procedimentos incrementais.

A seguir, são enumeradas as propostas de trabalhos futuros que dêem continuidade ao enriquecimento desta formulação:

1. empregar modelos anisótropos para a representação do maciço nãohomogêneo;

2. possibilitar que as diversas sub-regiões que representam o maciço possam ser dispostas em forma de nuvens tridimensionais no meio, e não apenas de maneira sobreposta como foi realizado neste trabalho;

3. considerar modelos viscoelásticos nas regiões mais críticas do meio;

4. simular o efeito de deslizamento entre as estacas e o maciço;

5. aplicar métodos mais eficientes em termos de tempo para se realizar as integrais de contorno;

6. considerar o efeito de segunda ordem no edifício;

7. modelar o edifício considerando as influências das lajes e do núcleo de rigidez;

Por fim, o trabalho representa uma poderosa ferramenta para o melhor entendimento do complexo sistema solo/estrutura. Na literatura é possível encontrar diversas formulações que tratam do assunto como um todo. Alguns modelos se detêm ao enriquecimento ou dos modelos do solo/subestrutura ou da superestrutura, mas sempre não possibilitando 
uma abordagem tão geral quanto este trabalho fez. É fato que em função da complexa ferramenta criada, não houve o devido tempo para se considerar modelos reológicos mais precisos do conjunto solo/subestrutura e/ou da superestrutura. Mas por si só este projeto fornece uma potente e frutífera base para aplicações no campo da interação solo/estrutura. 


\section{Referência}

ADELI, H. (1992). Parallel processing in computational mechanics. M. Dekker.

ADELI, H.; KAMAL, O. (1992). Concurrent analysis of large structures-l. Algorithms. Computers \& Structures, v. 42, n. 03, p. 413-424.

AIRD, T.J.; BATTISTE, E.L.; WALTON, C.G. (1977). Portability of mathematical software coded in Fortran. ACM Transactions on Mathematical Software (TOMS), v.3, n. 2, p.113-27, June.

ALIABADI, M.H.; HALL, W.S.; PHEMISTER, T.G. (1985). Taylor expansions for singular kernels in the boundary element method. Int. J. Numer. Meth. Engng, v.21, p. 2221-2236.

ALMEIDA, V.S. (1999). Uma adaptação do MEF para análise em multicomputadores: aplicações em alguns modelos estruturais. São Carlos. 126p. Dissertação (Mestrado) - Escola de Engenharia de São Carlos, Universidade de São Paulo.

ALMEIDA, V.S.; PAIVA, J.B. (2000). Improvements to classical preconditioners in finite element problems. In: TOPPING, B.H.V., ed. Finite elements: techniques and developments (Proc. $5^{\text {th }}$ International Conference on Computational Structures Technology $/ 2^{\text {nd }}$ International Conference on Engineering Computational Technology, Leuven, Belgium, 6-8 September). Edinburgh, Civil-Comp Press. p.43-49 (ISBN: 0-948749-67-9).

ALMEIDA, V.S.; PAIVA, J.B. (1999). Aplicação do método dos gradientes conjugados com o uso de pré-condicionadores em problemas do MEF. In: PIMENTA, P.M.; BRASIL, R.M.L.R.F.; ALMEIDA NETO, E.S., eds. Computational methods in engineering'99 (Iberian Latin-American Congress on Computational Methods in Engineering, 20. / Brazilian Congress on Computational Mechanics, 1., São Paulo, 3-5 Nov. 1999). [CD-ROM]. São Paulo, EPUSP, 18p.

ALMEIDA, V.A.F.; ÁRABE, J.N.C. (1991). Introdução à supercomputação. São Paulo, LTC. 
AMDAHL, G. (1967). The validity of the single processor approach to achieving large scale computing capabilities. In: Spring Joint Computer Conference, p. 483-485. (AFIPS Conference proceedings, v. 30).

ANTUNES, H.M.C.C.; IWAMOTO, R.K. (2000). Comparação entre resultados observados in situ e modelos numéricos para a interação solo-estrutura. In: Simpósio de Interação Estrutura-Solo em Edifícios. Programas de PósGraduação em Geotecnia e Engenharia de Estruturas da USP/SC.

AOKI, N.; LOPES, F.R. (1975). Estimating stress and settlements due to deep foundation. In. Conf. Panam. Soil Mechanics and Foundation Engineering, 05, p. 377-386.

ARGYRIS, J.H.; KELSEY, S (1960). Energy theorems and structural analysis. London, Butterworths.

ASSOCIAÇÃO BRASILEIRA DE NORMAS TÉCNICAS (1987). NBR 6123: Forças devidas ao vento em edificações.

ASSOCIAÇÃO BRASILEIRA DE NORMAS TÉCNICAS (1996). NBR 6122: Projeto e execução de fundações.

ASSOCIAÇÃO BRASILEIRA DE NORMAS TÉCNICAS (2000). NBR 6118: Projeto e Execução de Obras de concreto armado.

BANERJEE, P.K. (1976). Integral equation methods for analysis of piece-wise nonhomogeneous three-dimensional elastic solids of arbitrary shape. Int. J. Mechanical Science, v.18, p. 293-303.

BANERJEE, P.K. (1978). Analysis of axially and laterally loaded pile groups. Developments Soil Mechanics. London, Applied Science Publishers.

BANERJEE, P.K.; DAVIES, T.G. (1978). The behaviour of axially and laterally loaded single piles embedded in nonhomogeneous soils. Géotechnique, v. 28 , n. 3, p. 309-326.

BANERJEE, P.K.; DRISCOLL, P.M. (1976). Three-dimensional analysis of raked pile groups. Proc. Instn. Civ. Engrs., part. 2, v. 61, p. 653-671.

BANERJEE, P.K.; DAVIES, T.G. (1977). Analysis of pile groups embedded in Gibson soil. In: Int. Conf. Soil Mechs Fdn Engng., $9^{\text {th }}$, Tokyo. Proc., v.1, p. 381-386.

BARBIRATO, J.C.C. (1999). Método dos elementos de contorno com a reciprocidade dual para a análise transiente tridimensional da mecânica do 
fraturamento. São Carlos. 245p. Tese (Doutorado) - Escola de Engenharia de São Carlos, Universidade de São Paulo.

BARRAGY, E.; CAREY, G.F. (1988). A parallel element-by-element solution scheme. International Journal for Numerical Methods in Engineering, v. 26, p. 2367-2382.

BARROS, B. F. (2002). Métodos sem malha e método dos elementos finitos generalizados em análise não-linear de estruturas. São Carlos. 222p. Tese (Doutorado) - Escola de Engenharia de São Carlos, Universidade de São Paulo.

BATHE, K.J. (1982). Finite element procedures in engineering analysis. Englewood Cliffs, Prentice-Hall.

BATOZ, J.L. (1980). A study of three-node triangular plate bending elements. International Journal for Numerical Methods in Engineering, v. 15, p. 17711812.

BERGAN, P.G.; FELIPPA, C.A. (1985). A triangular membrane element with rotational degrees of freedom. Comp. Meths. in Appl. Mech. Eng., v.50, p.25-69.

BERGAN, P.G.; FELIPPA, C.A. (1986). Efficient implementation of a triangular membrane element with drilling freedoms. In: HUGHES, T.J.R.; HINTON, E. Finite element methods for plate and shell structures. Swansea,U.K.: cap.5, p.128-152.

BERGAN, P.G.; NYGARD, M. K. (1984). Finite elements with increased freedom in choosing shape functions. International Journal for Numerical Methods in Engineering, v. 20, p. 1771-1812.

BESKOS, D.E. (1987). Boundary element methods in mechanics. London, Computational Methods in Mechanics, v. 3, Elsevier Science Publishers.

BEZERRA, D.P. (1995). Análise de estruturas tridimensionais de edifícios altos considerando a rigidez transversal à flexão das lajes. São Carlos. 138p. Dissertação (Mestrado) - Escola de Engenharia de São Carlos, Universidade de São Paulo.

BITZARAKIS, S.; PAPADRAKAKIS, M.; KOTSOPULOS, A. (1997). Parallel solution techniques in computational structural mechanics. Comput. Methods Appl. Mech. Engr., v. 148, p. 75-104. 
BOOKER, J.R.; CARTER, J.P. ; SMALL, J.C. (1989). Some recent applications of numerical methods to geotechnical analysis. Computers \& Structures, v. 31, n.1, p. 81-92.

BOUASSIDA, M.; HADHRI, T. (1995). Extreme load of soils reinforced by columns: the case of an isolated column. Soils and Foundation, v. 35, p.2135.

BREBBIA, C.A. (1978). The boundary element method for engineers. London, Pentech Press.

BREBBIA, C.A.; DOMINGUEZ, J. (1989). Boundary elements. an introductory course. London, Computational Mechanics Publications.

BUDHU, M.; DAVIES, T.G. (1986). Analysis of laterally loaded piles in soft clays. Journal of Geotechnical Engineering, v. 114, n.1, p. 21-39.

BURMISTER, D.M. (1945). The general theory of stresses and displacements in layered systems I. Journal of Applied Physics, v.16, p.89-96.

BURMISTER, D. M. (1945). The general theory of stresses and displacements in layered systems III. Journal of Applied Physics, v.16, p.296-302.

BUTTERFIELD, R.; BANERJEE, P.K. (1971). The elastic analysis of compressible piles and pile groups. Géotechnique, v.21, n.1, p. 43-60.

BUTTERFIELD, R.; BANERJEE, P.K. (1971). The problem of pile group-pile cap interaction. Géotechnique, v.21, n.2, p. 135-142.

CARROLL, A.B.; WETHERALD, R.T. (1967). Application of parallel processing to numerical weather prediction. Journal of the ACM, v. 14, n. 3, p.591-614, July.

CHAN, K.S.; KARASUDHI, P.; LEE, S.L. (1974). Force at a point in the interior of a layered elastic half-space. Int. J. Solids Structs., v. 10, p. 1179- 1199.

CHEN, H. C.; BYREDDY, V. (1997). Solving plate bending problems using finite strips on networked workstations. Computers \& Structures, v.62, n.2, p.227236.

CHEUNG, Y.K.; THAM, L.G.; GUO, D.J. (1988). Analysis of pile group by infinite layer method. Géotechnique, v. 38,n. 3, p. 415-431.

CHEUNG, Y.K.; ZIENKIEWICZ, O.C. (1965). Plates and tanks on elastic foundations - an application of finite element method. Int. J. Solids Struct., v. 1, p. $451-461$. 
CHIN, J.T.; CHOW, Y.K. (1990). Numerical analysis of axially loaded vertical piles and pile groups. Computers and Geotechnics, v. 9, p. 273-290.

CHOW, Y.K. (1986). Discrete element analysis of settlement of pile groups. Computers \& Structures, v. 24, p. 157-166.

CHOW, Y.K.; TEH, C.I. (1991). Pile-cap-pile-group interaction in nonhomogeneous soil. Journal of Geotechnical Engineering , v. 117,n. 11, p. 1655-1668.

CIMERMAN, M. (1996). Resolução de sistemas lineares via métodos iterativos com pré-condicionadores - aplicação em problemas de engenharia de estruturas. São Paulo. 111p. Dissertação (Mestrado) - Escola Politécnica, Universidade de São Paulo.

CODA, H.B. (1993). Análise tridimensional transiente de estruturas pela combinação entre o método dos elementos de contorno e o método dos elementos finitos. São Carlos. 202p. Tese (Doutorado) - Escola de Engenharia de São Carlos, Universidade de São Paulo.

CODA, H.B. (2000). Contribuição à análise dinâmica de meios contínuos pelo método dos elementos de contorno. São Carlos. 349p. Tese (Livre-docência) - Escola de Engenharia de São Carlos, Universidade de São Paulo.

COOKE, R.W. (1986). Piled raft foundations on stiff clays - a contribution to design philosophy. Géotechnique, v. 36, n. 2, p. 169-203.

CRUSE, T.A. (1969). Numerical solutions in three-dimensional elastostatics. Int. J. Solids Struct., v.5, p. 1259-1274.

CRUSE, T.A. (1977). Mathematical foundations of the boundary integral equations method in solid mechanics. Report n0. AFORS-IR-1002, Prat and Whitney Aircraft Group.

CRUSE, T.A. (1974). An improved boundary-integral equation method for three dimensional elastic stress analysis. Computers \& Structures., v.4, p. 741-754.

CRUSE, T.A.; SHOW, D.W.; WILSON, R.B. (1977). Numerical solutions in axisymetric elasticity. Computers \& Structures, v.7, p. 445-451.

CUMINATO, J.A.; MENEGUETTE, M.JR. (1998). Discretização de equações diferenciais parciais: técnicas de diferenças finitas. Instituto de Ciências Matemáticas de São Carlos, Universidade de São Paulo. 
CUNHA, R.D.; HOPKINS, Tim (1998). PIM 1.1: The parallel iterative methods package for systems of linear equations user's guide (Fortran 77 version). Computing Laboratory, University of Kent at Canterbury, United Kingdom.

DAVIES, T.G.; BANERJEE, P.K. (1978). The displacement field due to a point load at the interface of a two layer elastic half-space. Géotechnique, v. 28, n.1, p. 43-56.

DeCEGAMA, A. L. (1989). Parallel processing architectures and VLSI hardware. Englewood Cliffs, Prentice Hall.

DEMMEL, J.W. (1993). Berkeley lecture notes on numerical linear algebra. mathematics Department and Computer Science Division, University of California.

DESAI, C.S.; ZAMAN, M.M. (1984). Thin-layer element for interfaces and joints. International Journal for Numerical Methods in Engineering, v. 8, p. 19-43.

DUARTE, C.A.; BABUSKA, I.; ODEN, J.T. (2000). Generalized finite element methods for three-dimensional structural mechanics problems. Computers \& Structures, v.77, p. 215-232.

DUBOIS, P.F.; GREENBAUM, A.; RODRIGUE, G.H. (1977). Aproximating the inverse of a matrix for use in iterative algorithms on vector processors. Computing, v.22, p. 257-268.

DUFF, I.S. (1977). MA28 - A set of FORTRAN subroutines for sparse unsymmetric linear equations. London, AERE Report R.8730, HMSO.

DUFF, I.S.; REID, J.K. (1982). A set of Fortran subroutines for solving sparse symmetric sets of linear equations. Report R10533, AERE Harwell.

DUFF, I.S.; ERISMAN, A.M.; REID, J.K. (1990). Direct methods for sparse matrices. Oxford, Claredon Press.

FARHAT, C.; ROUX, F.X. (1991). A method of finite element tearing and interconnecting and its parallel solution algorithm. Int. J. Numer, Methods Engrg., v. 32, p. 1205-1227.

FERRO, N.C.P (1993). Uma combinação MEC/ ME para análise de fundações enrijecidas por estacas. São Carlos. 135p. Dissertação (Mestrado) - Escola de Engenharia de São Carlos, Universidade de São Paulo.

FLYNN, M. (1966). Some computer organizations and their effectiveness. IEEE Transactions, v. 21. 
FRASER, R.A.; WARDLE, L.J. (1974). Numerical analysis of rectangular rafts on layered foundations. Géotechnique, v. 26, p. 613-630.

FREEMAN, L.; PHILIPS, C. (1992). Parallel numerical algorithms. New York, Prentice Hall.

GEIST, A.; BEGUELIN, A.; DONGARRA, J.; JIANG, W.;MANCHEK, R.; SUNDERAM, V. (1994). PVM 3 users's guide and reference manual. Engineering Physics and Mathematics Division, Mathematicals Sciences Section.

GIBSON, R.E. (1967). Some results concerning displacements and stresses in a non-homogeneous elastic half-space. Géotechnique, v. 17, p. 587-567.

GIBSON, R.E. (1974). The analytical method in soil mechanics. Géotechnique, v. 24, p. $115-140$.

GIBSON, R.E.; ALSI, G.S. (1974). The surface settlement of a linearly inhomogeneous cross-anisotropic elastic half-space. Journal of Applied Math. and Physics, v.25, p.843-847.

GOLUB, G.H.; VAN LOAN, C. F. (1984). Matrix computations. Baltimore, Johns Hopkins, University Press,.

GORBUNOV-POSSADOV, M.; SEREBRJANYI, R.V. (1961). Design of structures on elastic foundations. In: Conf. Soil Mechs Fdn Engng., $5^{\text {th }}$, Proc., v.1, p. 643-648.

GROSSO, A. Del; RIGHETTI, G. (1988). Finite element techniques and artificial intelligence on parallel machines. Computers \& Structures, v. 30, n.4, p. 9991007.

GUSMAO, A.D. (1990). Estudo da Interação Solo-estrutura e sua influência em recalques de edificações. 165p. Tese (Doutorado) - Universidade Federal do Rio de Janeiro, COPPE.

HARTMANN, F. (1980). Computing the C- matrix in non-smooth boundary points. In. BREBBIA, C.A., ed. New developments in boundary elements methods. Southampton, CMP Publ.

HETENYI, M.A. (1950). A general solution for the bending of beams on an elastic foundations of arbitrary continuity. Journal Applied Physics, v.21, p. 55-58. 
HOLANDA JR., O.G. (1998). Interação solo-estrutura para edifícios de concreto armado sobre fundações diretas. São Carlos. 191p. Dissertação (Mestrado) - Escola de Engenharia de São Carlos, Universidade de São Paulo.

HONG, D.C.; CHOW, Y.K.; YONG, K.Y. (1999). A method for the analysis of large vertically loaded pile groups. Int. J. for Numerical and Anal. Methods in Geomechanics, v.23, p. 243-262.

HUANG,Q.; CRUSE, T.A. (1993). Some notes on singular integral techniques in boundary element analysis. International Journal for Numerical Methods in Engineering, v. 36, p. 2643-2659.

JIANG, Y.; DAEBAE, F.; MARCHAL, J.M. (1997). Parallel computation of polymer melt flow through an extruder. Louvain-la-Neuve, Belgium. Polyflow S.A., Place de l'Université 16.

JOHNSON, O.G.; MICCHELLI, C.A.; PAUL, G. (1983). Polynomial preconditioners for conjugate gradient calculations. SIAM J. Num. Anal., v.20, p. 362-376.

JOHNSSON, S.L.; MATHUR, K.K. (1990). Data structures and algorithms for the finite element method on a data parallel supercomputer. International Journal for Numerical Methods in Engineering, v.29, p. 881-908.

JUN, L.; BEER, G.; MEEK, J.L. (1985). Efficient evaluation of integrals of order $1 / r, 1 / r^{2}, 1 / r^{3}$ using Gauss quadrature. Engineering Analysis, v. 2, p. 118-123.

KARYPIS, G.; KUMAR, V. (1995). Unstructured graph partioning and sparse matrix ordering system, version 2.0. Department of Computer Science , University of Minnesota.

KAVVADAS, M.; GAZETAS, G. (1993). Kinematic seismic response and bending of free-head piles in layered soil. Géotechnique, v. 43, n. 2, p. 207222.

KÉRISEL, J.; ADAM, M. (1967). Calcul des forces horizontales applicables aus fondations profondes dans les argiles et limons, Annales de I'Institut Technique du Batiment et des Travaux Publics, Paris, n. 239, p. 1653-1694.

KERR, A.D. (1964). Elastic and viscoelastic foundation models. Journal Applied Mechanic Transactions, ASME, v.31, p. 491-498.

KERR, A.D. (1965). A study of a new foundation model. Acta Mechanic, v.1, p. 135-147. 
KOLAR, V.; NEMEC, I. (1983). Complex automatization of calculations of technical structure interaction problems. In: Conf. SVTS, Kosice.

KRISHNA, P.Y.; SAM, C. (1992). Three-dimensional analysis of pile caps. Computers \& Structures, v. 42, n.3, p. 395-411.

LACHAT, J.C. (1975). A further development of the boundary integral technique for elastostatics. Ph.D. Thesis, University of Southampton.

LACHAT, J.C.; WATSON, J.O. (1976). Effective numerical treatment of boundary integral equations: a formulation for three-dimensional elastostatics. Int. J. for Numerical Methods in Engineering, v.10, p. 273-289.

LAW, K.H. (1986). A parallel finite element solution method. Computers \& Structures, v. 23, n. 6, p. 845-858.

LAW, K.H.; MACKAY, D.R. (1993). A parallel row-oriented sparse solution method for finite element structural analysis. International Journal for Numerical Methods in Engineering, v. 36, p. 2895-2919.

LAWSON , C.L.; HANSON, R.J.; KINCAID, D.; KROGH, F.T. (1979). Basic linear algebra subprograms for FORTRAN usage. ACM Trans. Math. Soft., p. 308-323.

LEE, S.L.; KOG, Y.C.; KARUNARATNE, G.P. (1986). Axiallly loaded piles in layered soil. Journal of Geotechnical Engineering, v.113,n. 4, April.

LEE, C.Y.; SMALL, J.C. (1991). Finite Layer Analysis of Laterally Loaded Piles in Cross-Anisotropic Soils. International Journal for Numerical Methods in Geomechanics, v. 15, p.785-808.

LEE, C.Y. (1993). Pile group settlement analysis by hybrid layer approach. Journal of Geotechnical Engineering, v.119, n.6, p. 984-997.

LEHMAN, M. (1966). A survey of problems and preliminary results concerning parallel processing and parallel processors. Proc. IEEE, v.54, p. 1889-1901.

LEITE, L.G.S.; CODA, H.B.; VENTURINI, W.S. (2001). Interação entre domínios bidimensionais e barras através do método dos elementos de contorno. In: XXI CILAMCE- CONGRESSO IBERO LATINO DE MÉTODOS COMPUTACIONAIS EM ENGENHARIA. Rio de Janeiro. p. 1-13.

LOGANATHAN, N.; POULOS, H.G.; XU, K.J. (2001). Ground and pile-group responses due to tunnelling. Soil and Foundations, v.41, p.51-67. 
LOLOI, M. (2000). Boundary integral equation solution of three-dimensional elastostatic problems in transversely isotropic solids using closed-form displacement fundamental solutions. International Journal for Numerical Methods in Engineering, v. 48, p. 823-842.

LOVE, A.E.H. (1944). A treatise on the mathematical theory of elasticity, 4. ed. New York, Dover.

LUENBERGER, D G. (1969). Optimization by vector space methods. New York, John Wiley.

MAIER, G.; NOVATI, G. (1987). Boundary element elastic analysis by a sucessive stiffness method. Int. J. for Numerical and Anal. Methods in Geomechanics, v.11, p. 435-447.

MATTES, N.S.; POULOS, H.G. (1969). Settlement of single compressible pile. Journal of the Soil Mechanics and Foundations Division, ASCE, v. 95, n.1, p. 189-207.

MEIJERINK, J.A.; VORST, H. A van der (1977). An iterative solution method for linear systems of which the coefficient matrix is a symmetric M-matrix. Mat. Comp., v.31, n.137, p.148-162.

MENDONÇA, A.V. (1997). Análise da interação placa-estaca-solo via combinação do método dos elementos finitos com o método dos elementos de contorno. São Carlos. 151p. Dissertação (Mestrado) - Escola de Engenharia de São Carlos, Universidade de São Paulo.

MENDONÇA, A.V. (2002). Estudo de estruturas compostas por lâminas planas: uma abordagem pelo método dos elementos de contorno. São Carlos. 291p. Tese (Doutorado) - Escola de Engenharia de São Carlos, Universidade de São Paulo.

MESQUITA, A.D. (1998). Uma formulação do método dos elementos finitos aplicada á analise elastoplástica de cascas. São Carlos. 144p. Dissertação (Mestrado) - Escola de Engenharia de São Carlos, Universidade de São Paulo.

MESQUITA, A.D.; CODA, H.B. (2000). Análise viscoelástica da interação soloestrutura, representação bidimensional. In: Simpósio de Interação EstruturaSolo em Edifícios. Programas de Pós-Graduação em Geotecnia e Engenharia de Estruturas da USP/SC.

MESSAFER, T.; COATES, L.E. (1989). An application of FEM/BEM coupling to foundation analysis. Advances in Boundary Methods. Eds. C.A. Brebbia \& 
Connor, Computational Mechanics Publications: Southampton and Boston, p. 211-221.

MINDLIN, R.D. (1936). Force at a point in the interior of a semi-infinite solid. J. Physics, v.7, p. 195-202.

MIRANKER, W.L. (1971). A survey of paralelism in numerical analysis. SIAM, v. 13 , n.4, p. 524-547.

MON-MA, M.L.; VENTURINI, W.S.; CODA, H.B. (1990). A simple technique to evaluate quasi-singular integrals. In: $1^{\text {st }}$. BRAZILIAN SEMINAR ON THE BOUNDARY ELEMENT METHOD IN ENGINEERING, Rio de Janeiro, Brasil.

MOURA, A.R.L.U. (1995). Interação solo-estrutura em edifícios. 135p. Dissertação (Mestrado) - Universidade Federal de Pernambuco, Recife.

MUSTOE, G.G. (1984). Advanced integration schemes over boundary elements and volume cells for two- and three-dimensional non-linear analysis. In: BANERJEE, P; MUKHERJEE, S. Developments in boundary element methods. London, Applied Science.

MUKHERJEE, S.; MORJARIA, M. (1984). On the efficiency and accuracy of the boundary element method and the finite element method. Int. J. for Numerical Methods in Engineering, v.20, p. 515-522.

MUQTADIR, A.; DESAI, C.S. (1986). Three-dimensional analysis of a pile-group foundation. Int. J. for Numerical and Anal. Methods in Geomechanics, v.10, p. 41-58.

MYLONAKIS, G.; GAZETAS, G. (1988). Settlement and additional internal forces of grouped piles in layered soil. Géotechnique, v. 48,n.1, p. 55-72.

NAKAGUMA, R.K. (1979). Three-dimensional elastostatics using the boundary element method. New York. Ph.D. Thesis, University of Southampton

NOOR, A.K.; PETERS, J.M. (1989). A partitioning strategy for efficient nonlinear finite element dynamic analysis on multiprocessor computers. Computers \& Structures, v.31, n.5, p. 795-810.

NOOR, A.K. (1997). New computing systems and future high-performance computing environment and their impact on structural analysis and design. Computers \& Structures, v.64, n.1, p.1-30.

NOOR, A.K.; HARTLEY, S.J. (1978). Evaluation of element stiffiness matrices on CDC STAR-100 computer. Computers \& Structures, v.9, p. 151-160. 
NOOR, A.K.; LAMBIOTTE, J.J. (1979). Finite element dynamic analysis on CDC STAR-100 computer. Computers \& Structures ,v. 10, p. 7-12.

ONATE, E.I.N. (1995). Calculo de estructuras por el metodo de elementos finitos. Centro Internacional de Métodos Numéricos en Engeniería, Barcelona, Espana.

OTTAVIANI, M. (1975). Three-dimensional finite element analysis of vertically loaded pile groups. Géotechnique, v. 25, n.2, p. 159-174.

PAIVA, J. B. (1993). Formulação do método dos elementos de contorno para análise da interação solo-estrutura. São Carlos. 183p. Tese (Livre-docência) - Escola de Engenharia de São Carlos, Universidade de São Paulo.

PAIVA, J.B.; BUTTERFIELD, R. (1997). Boundary element analysis of plate-soil interaction. Computers \& Structures, v.64, p. 319-328.

PAN, E. (1997). Static Green's functions in multilayered half spaces. Applied Math. Modelling, v. 21, p. 509-521.

PARÍS, F.; CAÑAS, J. (1997). Boundary element method: fundamental and applications. New York. Oxford University Press.

PETELEIRO, S. C. (1996). Utilização da formulação livre para desenvolvimento de um elemento de membrana com liberdades rotacionais. São Carlos. 101p. Dissertação (Mestrado) - Escola de Engenharia de São Carlos, Universidade de São Paulo.

POULOS, H.G. (1967). Stresses and displacements in an elastic layer underlain by rough rigid base. Géotechnique, v.17,p. 378-410.

POULOS, H.G. (1968a). Analysis of the settlement of pile groups. Géotechnique, v. 18, p. 449-471.

POULOS, H.G. (1971a). Behaviour of laterally loaded piles: I - single piles. Journal of the Soil Mechanics and Foundations Division, ASCE, v. 97, n.SM5, p. 711-731.

POULOS, H.G. (1971b). Behaviour of laterally loaded piles: II - pile groups. Journal of the Soil Mechanics and Foundations Division, ASCE, v. 97, $\mathrm{n}$. SM5, p. 733-751.

POULOS, H.G. (1981). Soil - structure interaction - general report. p. 307-334.

POULOS, H.G. (1989). Pile behaviour - theory and application. Géotechnique, v. 39, p. $365-415$. 
POULOS, H.G. (1994). An approximate numerical analysis of pile-raft interaction. Int. J. for Numerical and Anal. Methods in Geomechanics, v.18, p. 73-92.

POULOS, H.G. (1979). Settlement of single piles in nonhomogeneous soil. Journal of Geotechnical Engineering Division, ASCE, v. 105,n. 5, p. 627-641.

POULOS, H.G.; DAVIS, E.H. (1968b). The settlement behaviour of single axially loaded incompressible piles and piers. Géotechnique, v. 18, p. 351-371.

POULOS, H.G.; DAVIS, E.H. (1974). Elastic solutions in and soil and rock mass. New York, John Wiley \& Sons 535p.

POULOS, H.G.; DAVIS, E.H. (1980). Pile foundation analysis and design. New York, John Wiley \& Sons.

PRZEMIENIECKI, J.S. (1962). Matrix structural analysis of substructures. AIAA Journal, v.1, n.1, p. 138-147.

RAO, A.R.M.; LOGANATHAN, K.; RAMAN, N.V. (1993). Studies on two concurrent solvers for finite element analysis. Advances in Engineering Software, v. 18, p. 161-166.

RANDOLPH, M. F., WROTH, C. P. (1979). An analysis of the vertical deformation of pile groups. Géotechnique, v. 29, p. 423-439.

REDDY, J.N. (1984). Energy and variacional methods in applied mechanics. New York, Wiley Interscience.

REIS, J.H.C. (2000). Interação solo-estrutura de grupos de edifícios com fundações superficiais em argila mole. São Carlos. 148p. Dissertação (Mestrado) - Escola de Engenharia de São Carlos, Universidade de São Paulo.

RICCARDELLA, P.C. (1973). An implementation of the boundary integral technique for planar problems in elasticity and elastoplasticity. Report no. SM-73-10, Depto. Mech. Eng. Carnegie Mellon University, Pittsburg.

RIOS, B. M. C. (1991). Análise tridimensional e envoltória de esforços em edifícios altos sujeitos à ações verticais e laterais. São Carlos. 247p. Dissertação (Mestrado) - Escola de Engenharia de São Carlos, Universidade de São Paulo.

RIZZO, F.L. (1967). An integral equation approach to boundary value problems of classical elastostatics. Quart. Appl. Math.,v.25, p.83-95. 
ROMANEL, C.; KUNDU, T. (1990). Soil-structure interaction in a layered medium. Int. J. Engng. Sci., v.28, p. 191-213.

ROMANEL, C.; AEDO, J.L.C; MOTTA, L.G. (1997). Uma análise tridimensional de pavimentos flexíveis. In: XVIII CILAMCE - CONGRESSO IBERO LATINO DE MÉTODOS COMPUTACIONAIS EM ENGENHARIA. BRASÍLIA - DF Anais do XVIIII CILAMCE. Editora da UnB: v. 1, p. 1995-2002.

ROMANEL, C.; VEIGA, J.; ROEHL, D. (2000). Aplicação da transformada de Fourier na análise de problemas solo-estrutura tridimensionais. In: XXI CILAMCE- CONGRESSO IBERO LATINO DE MÉTODOS COMPUTACIONAIS EM ENGENHARIA. Rio de Janeiro.

SAAD, Y.; SCHULTZ, M.H. (1986). GMRES: A generalized minimal residual algorithm for solving nonsymmetric linear systems. SIAM J. Sci. Stat. Comput., v.7, n.7, p. 856-869.

SADECKA, L. (2000). A finite/infinite element analysis of thick plate on a layered foundation. Computers \& Structures, v. 76, p. 603-610.

SAMPAIO, M.C.; SAUÉ, J.P.; MOURA, J. B. (1988). Unix - guia do usuário. São Paulo, Makron Books do Brasil.

SAVASSI, W. (1996). Introdução ao método dos elementos finitos : em análise linear de estruturas. São Carlos, Escola de Engenharia de São Carlos, Universidade de São Paulo.

SAXENA, M.; PERUCCHIO, R. (1992). Parallel fem algorithms based on recursive spatial decomposition - I. Automatic mesh generation. Computers \& Structures, v. 45, n. $5 / 6$, p. $817-831$.

SCHMIT, L.A.JR.; LAI, Y.C. (1994). Structural optimization based on preconditioned conjugate gradient analysis methods. International Journal for Numerical Methods in Engineering, v. 37, p. 943-964.

SIMO, J.C.; FOX, D.D.; RIFAI, M.S. (1989). On a stress resultant geometrically exact shell model. Part II: The linear theory; computational aspects. Computer Methods in Applied Mechanics and Engineering, v.73, p. 3-92.

SMALL, J.C.; BOOKER, J.R. (1984). Finite layer analysis of layered elastic materials using a flexibility approach. Part 1 - Strip loadings. Int. J. for Numerical Methods in Engineering, v. 20, p. 1025-1037.

SOUSA JR, E. (1996). Um aplicativo para o ambiente windows para aquisição de dados para análise de pavimentos de edifícios via métodos dos elementos 
finitos. São Carlos. 99p. Dissertação (Mestrado) - Escola de Engenharia de São Carlos, Universidade de São Paulo.

SOUTHCOTT, P.H.; SMALL, J.C. (1996). Finite layer analysis of vertically loaded piles and pile groups. Computer and Geotechnics, v. 18, n.1, p. 47-63.

SOUZA, V.J. B.; ALMEIDA, F.P.A.; CODA, H.B. (2002). A general study of source location for BEM elasticity. In: JOINT CONFERENCE OF ITALIAN GROUP OF COMP. MECH. AND IBERO-LATIN AMERICAN ASSOCIATION OF COMP. METHODS IN ENG., $3^{\text {rd }}$, Giulianova, Italia.

SUN, C.T. , CHIEN, L.S. (1994). Parallel Computations in solid mechanics. p. 245-295.

TA, L.D.; SMALL, J.C. (1998). Analysis and performance of piled raft foundations on layered soils-case studies. Soil and Foundations, v.38, n.4, p. 145-150.

TEJERINA, E. C. (1996). Sobre o uso do MEC para o estudo de interação de placas com o meio contínuo. São Carlos. 247p. Tese (Doutorado) - Escola de Engenharia de São Carlos, Universidade de São Paulo.

TELLES, J.C.F. (1987). A self-adaptative co-ordinate transformation for efficient numerical evaluation of general boundary element integrals. Int. J. for Numerical Methods in Engineering, v.24, p. 959-973.

TIMOSHENKO, S.P.; WOINOWSKI-KRIEGER, S. (1959). Theory of plates and shells. McGraw-Hill, Tokyo.

TOPPING, B.H.V.; KHAN, A.I. (1996). Parallel finite element computations. Edinburgh, Saxe-Coburg Publications.

TOPPING, B.H.V.; KHAN, A.I.; BAHREININEJAD, A. (1997). Parallel training of neural networks for finite element mesh decomposition. Computers \& Structures, v.63, p. 693-707.

TUTORIAIS do CISC (1998). http://www.cisc.sc.usp.br/tutoriais/ (1 Agosto)

TUTORIAIS do CISC (1998). http://www.cisc.sc.usp.br/tutoriais/ (1 Agosto)

TUTORIAL do IBM SP (1998). http://www.cisc.sc.usp.br/servicos/sp2.html (30 Julho).

VENTURINI, W.S. (1988). Um estudo sobre o método dos elementos de contorno e suas aplicações em problemas de engenharia. São Carlos. 349p. 
Tese (Livre-docência) - Escola de Engenharia de São Carlos, Universidade de São Paulo.

WANG, C.M.; IANG, Y.X.; WANG, Q. (2001). Axisymmetric buckling of reddy circular plates on Pasternak foundation. Journal Engineering Mechanic, ASCE, v.127, p. 254-259.

WARDLE, L.J.; FRASER, R.A. (1974). Finite element analysis of a plate on a layered cross-anisotropic foundation. In: INT. CONF. FINITE ELEMENT METHODS ENGNG., $1^{\text {st }}$, University of NSW.

WALSHAW, C.; CROSS, M.; EVERETT, M.G. (1995). A Parallelisable Algorithm for Optimising Unstructured Mesh Partitions. http:// www.gre.ac.uk/ wc06/papers/walshaw95.ps.gz (1 Agosto)

WHITAKER, T. (1957). Experiments with model piles in groups. Géotechnique, v.7, n.4, p. 147-167.

WITT, M. (1984). Solutions of plates on a heterogeneous elastic foundation. Computers \& Structures, v.18, p. 41-45.

XU, K.J.; POULOS, H.G. (2000). General elastic analysis of piles and pile groups. Int. J. for Numerical and Anal. Methods in Geomechanics, v.24, p. 1109-1138.

ZOIS, D. (1988a). Parallel processing techniques for FE analysis: stiffiness, loads and stresses evaluation. Computers \& Structures, v.28, p. 247-260.

ZOIS, D. (1988b). Parallel processing techniques for FE analysis: system solution. Computers \& Structures, v.28, p. 261-274. 